\title{
Nanocellulose from the Appalachian Hardwood Forest and Its Potential Applications
}

\author{
Masoumeh Hassanzadeh
}

Follow this and additional works at: https://researchrepository.wvu.edu/etd

\section{Recommended Citation}

Hassanzadeh, Masoumeh, "Nanocellulose from the Appalachian Hardwood Forest and Its Potential Applications" (2018). Graduate Theses, Dissertations, and Problem Reports. 5780.

https://researchrepository.wvu.edu/etd/5780

This Dissertation is protected by copyright and/or related rights. It has been brought to you by the The Research Repository @ WVU with permission from the rights-holder(s). You are free to use this Dissertation in any way that is permitted by the copyright and related rights legislation that applies to your use. For other uses you must obtain permission from the rights-holder(s) directly, unless additional rights are indicated by a Creative Commons license in the record and/ or on the work itself. This Dissertation has been accepted for inclusion in WVU Graduate Theses, Dissertations, and Problem Reports collection by an authorized administrator of The Research Repository @ WVU.

For more information, please contact researchrepository@mail.wvu.edu. 


\title{
NANOCELLULOSE FROM THE APPALACHIAN HARDWOOD FOREST AND ITS POTENTIAL APPLICATIONS
}

Masoumeh Hassanzadeh

\author{
Dissertation submitted to the \\ Davis College of Agriculture, Natural Resources and Design \\ at West Virginia University \\ in partial fulfillment of the requirements for the degree of \\ Doctor of Philosophy in \\ Forest Resources Science
}
Gloria S. Oporto, Ph.D., Committee Chair
Benjamin Dawson-Andoh, Ph.D.
Bjorn Soderberg, Ph.D.
Yon Rojanasakul, Ph.D.
Sushant Agarwal, Ph.D.
School of Natural Resources

Morgantown, West Virginia

2018

Keywords: Appalachian hardwood, Nanocellulose, Nanofibrillated cellulose, TEMPO nanofibrillated cellulose Copyright 2018 Masoumeh Hassanzadeh 


\section{ABSTRACT \\ NANOCELLULOSE FROM THE APPALACHIAN HARDWOOD FOREST AND ITS POTENTIAL APPLICATIONS}

\section{Masoumeh Hassanzadeh}

Nanofibrillated cellulose (NFCs) are nanoscale fibers of high aspect ratio that can be isolated from a wide variety of cellulosic sources, including wood and bacterial cellulose. With high strength despite of their low density, NFCs are a promising renewable building block for the preparation of nanostructured materials and composites. To fabricate NFC-based materials with improved mechanical and chemical properties and additional new functionalities for different applications, it is essential to tailor the surface properties of individual NFCs. The surface structures control the interactions between NFCs and ultimately dictate the structure and macroscale properties of the bulk material. This research was focused on determining the feasibility of using hardwood residues from the Appalachian Hardwood Forest for the production of nanofibrillated cellulose (NFC). In addition, some modifications during the NFC production process were performed to evaluate their improvement to incorporate more antimicrobial copper in the cellulosic backbone. This thesis has been divided in the following main chapters: 1) Literature review regarding to nanocellulosic materials and their production processes, 2) Nanocellulose current and potential applications, 3) Nanofibrillated cellulose from the Appalachian Hardwood logging residues, 4) Modified nanofibrillated from the Appalachian Hardwood logging residues, 5) Preparation of nanocellulose using ionic liquids - A review, 6) Nanocellulose-based drug delivery system - A review, 7) Safety aspects on the utilization of lignocellulosic based materials - A review. 


\section{ACKNOWLEDGMENTS}

I wish to thank all the people that made this thesis possible, and that supported me over the course of the past three years at West Virginia University (WVU).

I am very grateful to my supervisor, Associate Professor Dr. Gloria Oporto for her support and for the opportunity to perform this instructive $\mathrm{PhD}$ thesis and for providing me with the opportunity to engage in this project.

Sincere appreciation is also to Dr. Ben Dawson, Dr. Bjorn Soderberg, Dr. Yon Rojanasakul, and Dr. Sushant Agarwal, members of my academic committee, for agreeing to serve on my committee and for their advice and suggestions helping me to sharpen the focus of my research. I will be remiss without saying a special thank you to Dr. Ben Dawson for his invaluable friendship and mentorship to me over my staying at WVU. Many thanks to USDA Forest Service, Forest Products Laboratory where I was allowed to perform some experiments, and Dr. Ronald Sabo, Alan Rudie and Richard Reiner for their help and valuable constructive feedback.

I also would like to express my gratitude to all whom, directly or indirectly, have helped me in this adventure, including Dean Daniel Robison, David Stewart, Catherine Yura and Doina Jickich.

I acknowledge the funding for this work that was provided by NIFA McStennis WVA00098 "Efficient utilization of biomass for biopolymers in central Appalachia”, and USDA NIFA Grant No. 2013-34638-21481 “Development of novel hybrid cellulose nanocomposite film with potent biocide properties utilizing low quality Appalachian hardwoods”.

Finally, thanks are due to entire family for their love and moral support, specially dedicated to my mom and my sister, the best friends and the hidden strength behind my every success. 


\section{Table of Contents}

ACKNOWLEDGMENTS ...........................................................................................................iii

LIST OF FIGURES ..........................................................................................................................

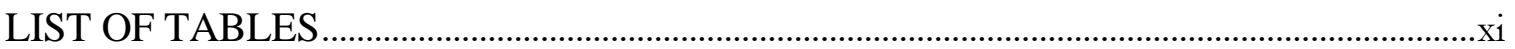

LIST OF SYMBOLS/abbreviation / NOMENCLATURE ...........................................................ii

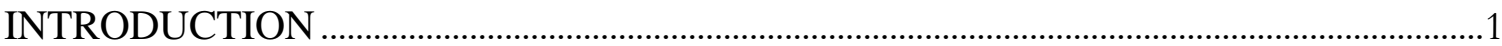

CHAPTER 1: LITERATURE REVIEW NANOCELLULOSE ………………………………..... 10

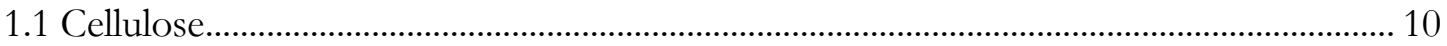

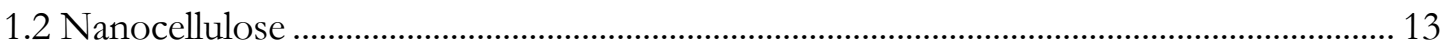

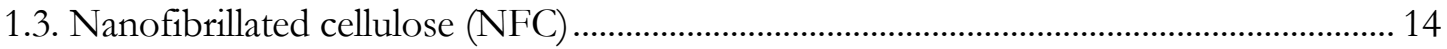

1.4. Cellulose nanocrystals $(\mathrm{CNC})$...................................................................................... 15

1.5 Bacterial nanocellulose (BNC) ………………………………………………………... 16

1.6 Nanocellulose preparation methods .................................................................................... 16

1.6.1. Top-down approaches to produce nanocellulose ....................................................... 17

1.6.2. Bottom-up approaches to produce nanocellulose................................................... 19

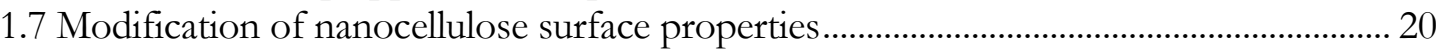

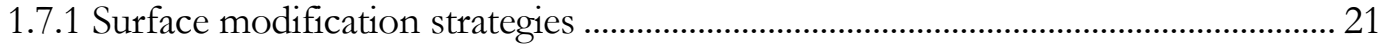

1.7.2 TEMPO - mediated oxidation ……………………………………………………. 24

1.8 Potential Applications of TEMPO-oxidized Celluloses....................................................... 27

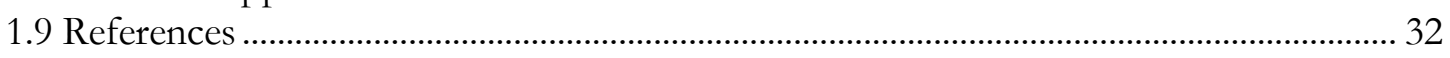

CHAPTER 2: NANOCELLULOSE CURRENT POTENTIAL APPLICATIONS............... 37

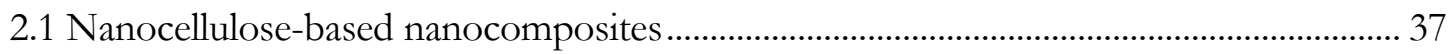

2.2 Sensors (medical, environmental, and industrial) ………………………………………..... 39

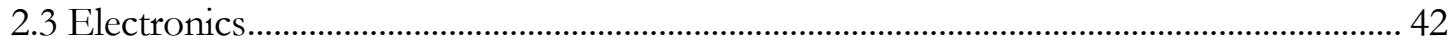

2.4 Antibacterial/Antimicrobial........................................................................................ 45

2.5 Biomedical Applications................................................................................................... 48

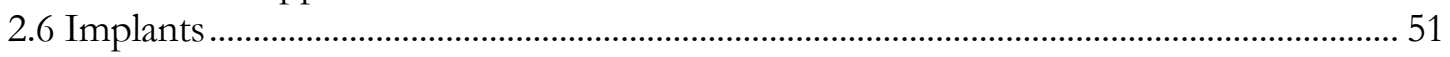

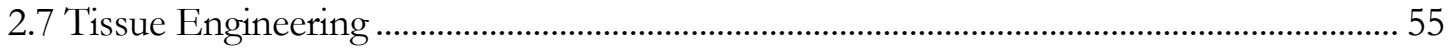

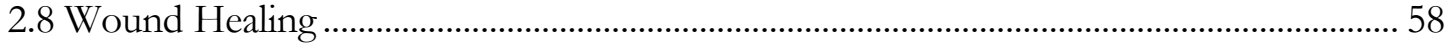

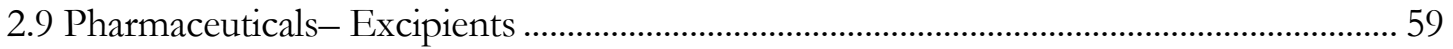

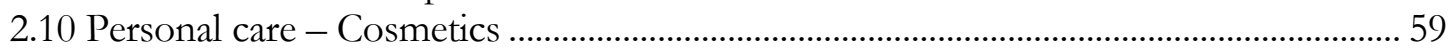

2.11 Nanocellulose paper and film......................................................................................... 59

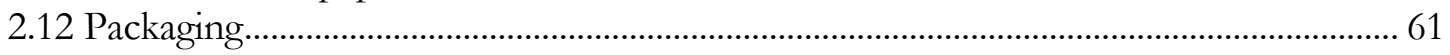

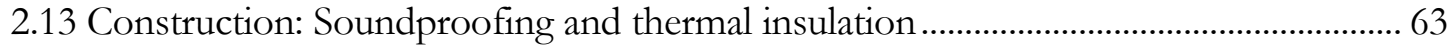

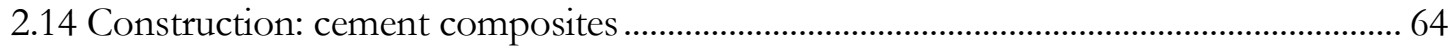

2.15 Structural: Automotive and Aerospace ………………………………………………… 64

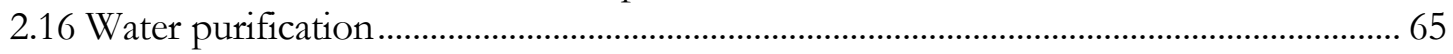

2.17 Textiles - clothing ……………………………………………………………………… 65

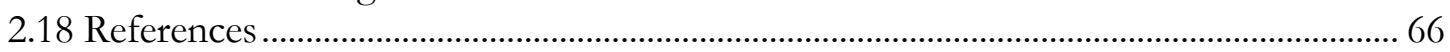

CHAPTER 3: NANOFIBRILLATED CELLULOSE FROM APPALACHIAN HARDWOODS LOGGING RESIDUES ................................................................................. 75

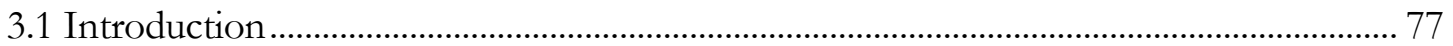

3.2 Materials and Methods.................................................................................................... 79

3.2.1 Materials ........................................................................................................... 79

3.2.2 Methods............................................................................................................ 79

3.2.2.13 Preparation of hybrid TNFC and copper nanoparticles ................................... 85 
3.3 Results and Discussion ..................................................................................................... 85

3.3.1 Chemical analysis .................................................................................................. 85

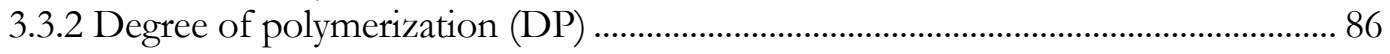

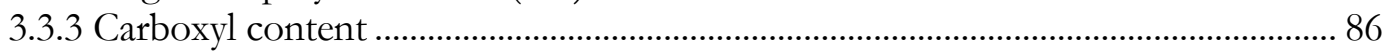

3.3.4 FTIR spectroscopy analysis ..................................................................................... 86

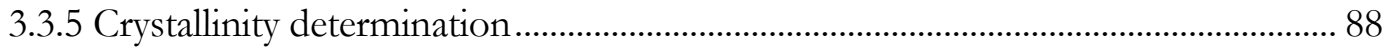

3.3.6 Thermal gravimetric analysis ...................................................................................... 89

3.3.7 Morphology of the Treated and Untreated samples................................................. 90

3.3.8 Mechanical properties of NFC films ................................................................... 91

3.3.9 Copper determination.................................................................................................. 92

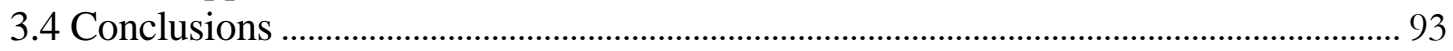

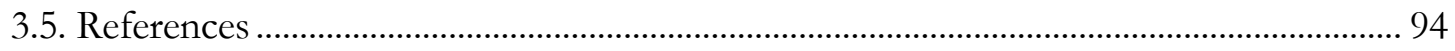

CHAPTER 4: MODIFIED NANOFIBRILLATED FROM THE APPALACHIAN HARDWOOD LOGGING RESIDUES.................................................................................. 108

4.1 Introduction ................................................................................................................ 110

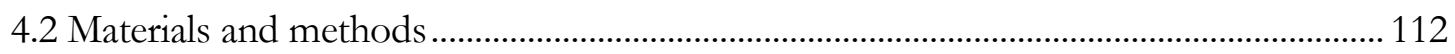

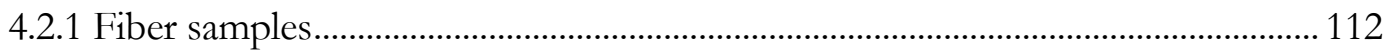

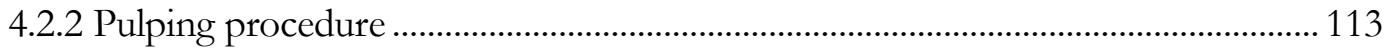

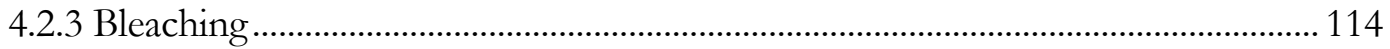

4.2.4 TEMPO-Mediated Oxidation.................................................................................. 114

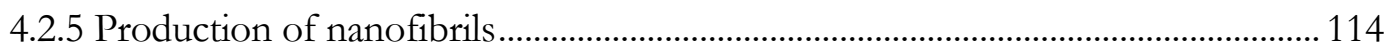

4.2.6 Fiber chemical composition .................................................................................... 115

4.2.7 Intrinsic viscosity and degree of polymerization .................................................... 115

4.2.8 Carboxyl content of the pulp ……………………………………………………... 115

4.2.9 Fourier transform infra-red spectroscopy (FTIR) ................................................... 116

4.2.10 X-ray diffraction (XRD) characterization .............................................................. 116

4.2.11 Thermogravimetric analysis (TGA) ..................................................................... 116

4.2.12 Morphological characterization ............................................................................... 116

4.2.13 Preparation of hybrid TNFC and copper nanoparticles....................................... 117

4.2.14 Copper determination on TNFC from Red oak and Yellow poplar................... 118

4.3 Results and discussion....................................................................................................... 118

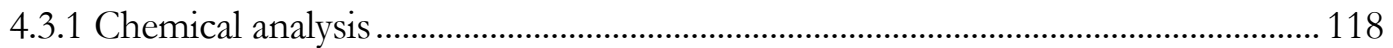

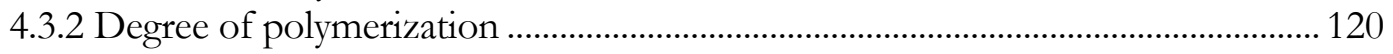

4.3.3 Carboxyl content .................................................................................................. 121

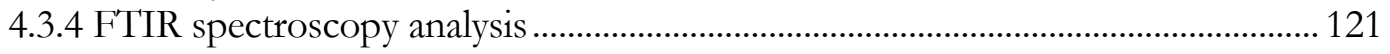

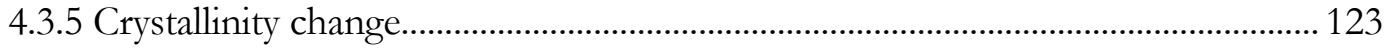

4.3.6 Thermal gravimetric analysis .................................................................................. 123

4.3.7 Morphology of the treated and untreated samples ……………………………….. 125

4.3.8 Mechanical test results ................................................................................................ 126

4.3.9 Copper determination.......................................................................................... 126

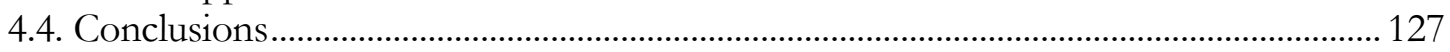

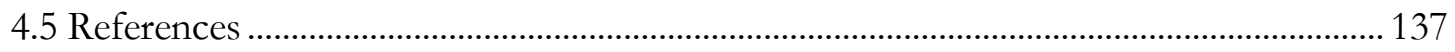

CHAPTER 5: PREPARATION OF NANOCELLULOSE USING IONIC LIQUIDS - A

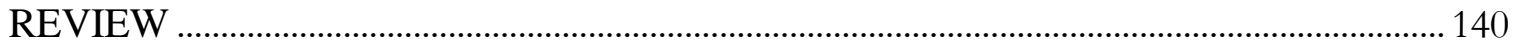

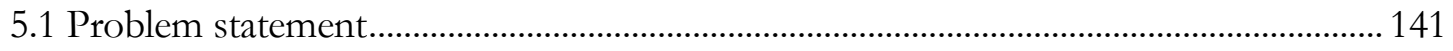

5.2 Ionic Liquids (ILs) …………………………………………………………………. 145 
5.2.1 Advantages of ILs

5.2.2 Dis-advantages of ILs

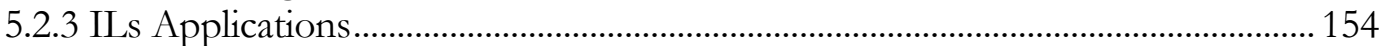

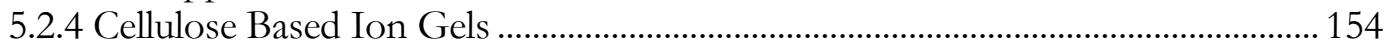

5.2.5 Fraction of lignocellulosic materials.......................................................................... 157

5.2.6 Preparation of cellulose derivatives......................................................................... 157

5.2.7 Preparation of cellulose composites ............................................................................. 157

5.2.8 Polymerized Ionic Liquid-Cellulose Composites ....................................................... 159

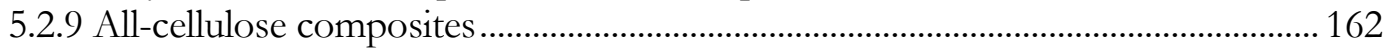

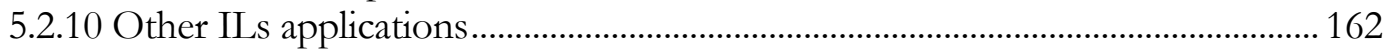

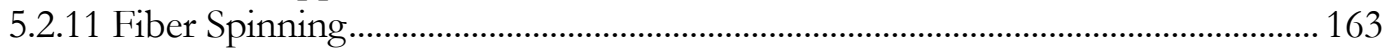

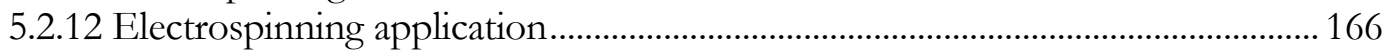

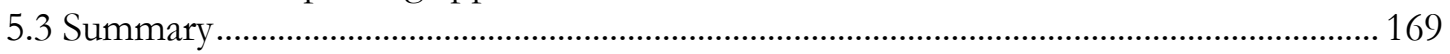

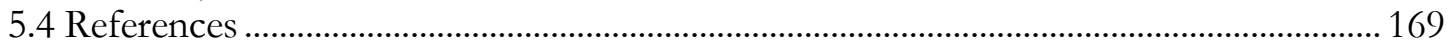

CHAPTER 6: NANOCELLULOSE-BASED DRUG DELIVERY SYSTEM - A

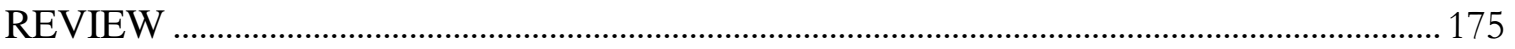

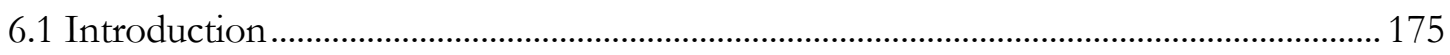

6.2 Nanofibrillated cellulose in protein and gene therapy …..................................................... 177

6.3 Nanocellulose in Nanoparticulate Drug Delivery .............................................................. 179

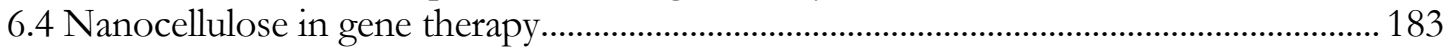

6.5 Nanocellulose in Microparticulate Drug Delivery ................................................................. 187

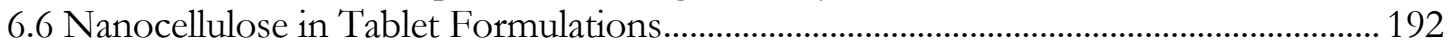

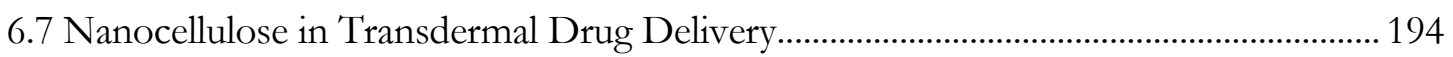

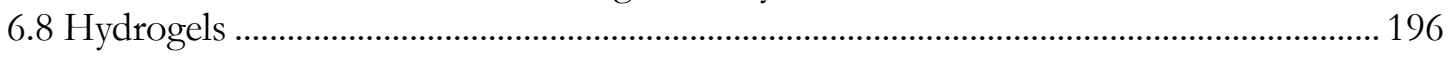

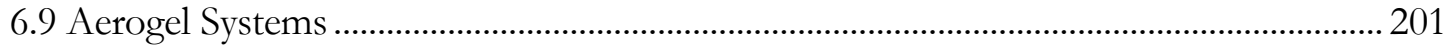

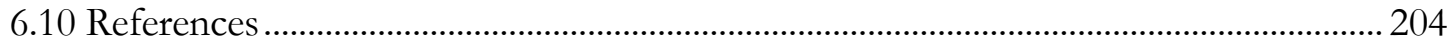

CHAPTER 7: SAFETY ASPECTS ON THE UTILIZATION OF LIGNOCELLULOSIC

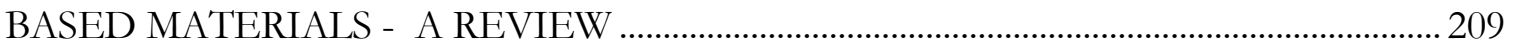

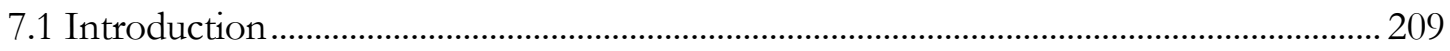

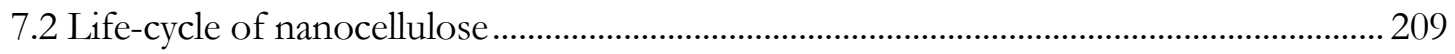

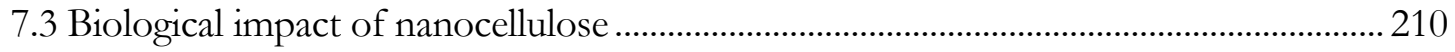

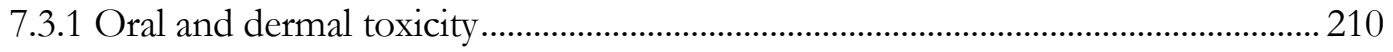

7.3.2 Cytotoxicity effect of cellulose based nanoparticles .................................................. 210

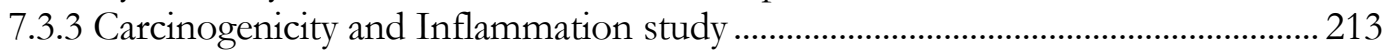

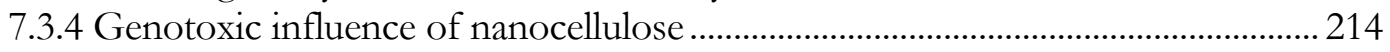

7.4 Safety and regulatory issues of nanocellulose in food........................................................ 215

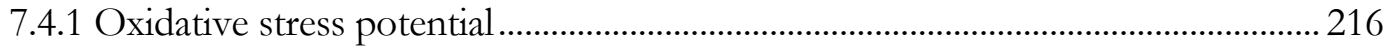

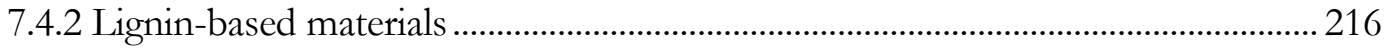

7.5 Summary

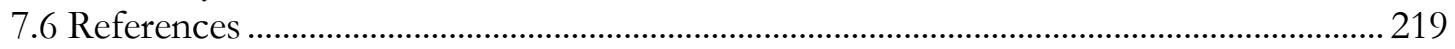




\section{LIST OF FIGURES}

Figure 1.1 Schematic representation of (a) single cellulose chain repeat unit, showing the directionality of the 1-4 linkage and intrachain hydrogen bonding (dotted line), (b) idealized cellulose microfibril showing one of the suggested configurations of the crystalline and amorphous regions, and (c) cellulose nanocrystals after acid hydrolysis dissolved the disordered regions (Moon et al. 2011).

Figure 1.2 Schematic illustration of the hierarchical structure of cellulose in wood and cellulose nanoparticles isolation (Postek et al. 2010)

Figure 1.3 Simplified illustration of the hierarchical organization of cellulose in the wood cell wall (Lavoine et al. 2012).

Figure 1.4 Transmission electron micrographs of a) MFC and b) NCC; c) scanning electron micrograph of BNC (Klemm et al. 2011)........................................................................... 16

Figure 1.5 Classification of nanocellulose fabrication approaches (adapted from Li, 2012)...... 17

Figure 1.6 Cellulose functionalized with anionic carboxymethyl groups (a) and quaternary

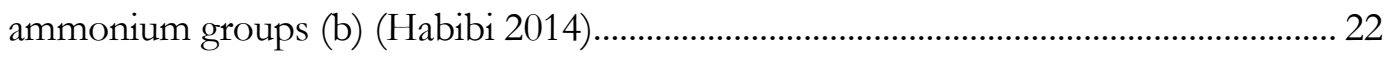

Figure 1.7 A scheme of wood acetylation mechanism (Khalil et al. 2014)..................................... 23

Figure 1.8 Examples of chemical modification of nanocellulose.................................................... 23

Figure 1.9 TEMPO oxidization of cellulose (Isogai and Kato 1998)............................................. 24

Figure 1.10 Reaction scheme of TEMPO-mediated oxidation of primary hydroxyls (Iwamoto et al. 2010) 25

Figure 1.11 Schematic representation of a $\mathrm{CNC}$ cross section illustrating the selective TEMPOmediated oxidation of primary hydroxyl groups at the surface of the crystal (Habibi et al, 2014) ......................................................................................................................... 26

Figure 1.12 Possible mechanisms of formation of inter-fiber hemiacetal linkages and improvement of wet-tensile strength of the sheets prepared from the TEMPO-oxidized cellulose(Saito and Isogai 2006). 30

Figure 1.13 Conversion from TOCN/water dispersion to diverse bulk materials (Isogai 2013) 31

Figure 2.1 (a) Concept of wirelessly driven EAPap actuator, and (b) Configuration of wirelessly driven CPIL actuator (Yang et al. 2010)........................................................................... 38

Figure 2.2 Cellulose based supercapacitor and battery (Pushparaj et al. 2007)............................. 43

Figure 2.3 Synthesis of FITC-Labeled, Folic Acid-Conjugated CNCs (Dong et al. 2014) ........ 50

Figure 2.4 Cell morphology of the (i) pristine MFC. MFC/CPO-x with different $\mathrm{CPO}$ concentrations: (ii) 5 , (iii) 10 , and (iv) $15 \mathrm{wt} \%$. MFC/[CPO1catalase] with different CPO concentrations: (v) 5, (vi) 10, and (vii) 15\% w/w. Scale bar540 lm. Reproduced with permission from (Chang and Wang 2013)........................................................................ 51

Figure 2.5 Hypothetical model of the stiffness change mechanism in the sea cucumber dermis

(Takehana et al. 2014)............................................................................................................. 53

Figure 3.1 (a) FT-IR spectra of Red oak: (I)Raw material, (II)Bleached, (III)Tempo-oxidized Wood pulp (TOWP) and (IV)Tempo-oxidized cellulose nanofibers (TNFC). (b) FT-IR 
spectra of Yellow poplar: (I)Raw material, (II)Bleached, (III)Tempo-oxidized wood pulp (TOWP) and (IV)Tempo-oxidized cellulose nanofibers (TNFC) ................................. 101

Figure 3.2 X-ray diffraction patterns of: a) Raw R.O,TOWPR.O, and TNFCR.O .................. 102

Figure 3.3 Thermogravimetric (TGA (a)) and the corresponding derivative thermogravimetric (DTG) (DTG (b)) curves of the raw material and TEMPO-oxidized cellulose nanofibers (TNFC) 103

Figure 3.4 FE-SEM micrographs of the bleached R.O (a) and Y.P (b);TEMPO oxidized wood pulp (TOWP) of R.O (c) and Y.P (d); TEMPO nanofibrillated cellulose (TNFC) of R.O (e) and Y.P (f) samples after a solvent exchange(E/tert-B-FD treatment) followed by freeze drying. 104

Figure 3.5 TEM images of $\mathrm{TNFC}_{\mathrm{R} . \mathrm{O}}$ (a) and $\mathrm{TNFC}_{\mathrm{Y} . \mathrm{P}}(\mathrm{b})$ and their size distribution properties( c: R.O), (d: Y.P). 105

Figure 3.6 Main tensile properties of NFC films prepared from cellulose nanofibrils of R.O (R.O) and Y.P (Y.P). (a) Stress; (b) Strain at break; (c) Tensile modulus. 106

Figure 3.7 SEM images of dried TNFCRO after the treatment with copper sulfate and reducing agent sodium borohydride (TNFCR.O-Cu). 107

Figure 4.1 Reaction scheme for surface modification of cellulose with TEMPO-mediated oxidation (Carlsson et al. 2014). 111

Figure 4.2 Upper figure: FT-IR spectra of: (a)Raw material, (b) Bleached, (c) Tempo-oxidized cellulose nanofibers (TNFC) of Red oak (R.O.); Lower figure: FT-IR spectra of: (a) Raw material, (b) Bleached, (c) Tempo-oxidized cellulose nanofibers (TNFC) of Yellow poplar (Y.P.)

Figure 4.3 X-ray diffraction patterns of: Bleached, TOWP, and TNFC samples of R.O (left) and Y.P (right)

Figure 4.4 FE-SEM micrographs of the bleached R.O (a) and Y.P (b);TEMPO-oxidized wood pulp (TOWP) of R.O (c) and Y.P (d); TEMPO nanofibrillated cellulose and Copper $\left(\mathrm{TNFC}_{\mathrm{RO}}+\mathrm{Cu}\right)$ of R.O (e) and Y.P (f) samples. 134

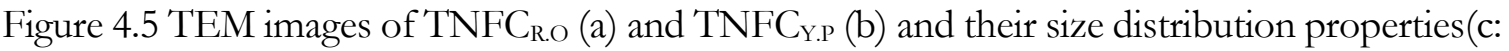
R.O), (d: Y.P) 135

Figure 4.6 Thermogravimetric (TGA (a)) and the corresponding derivative thermogravimetric (DTG (b)) curves of the raw material and TEMPO-oxidized cellulose nanofibers (TNFC). 136

Figure 5.1 Cellulose simplified as a cellobiose. Equatorial hydrogen bonds arrange cellulose chains in sheets that are stacked onto each other in three-dimensional crystals by means of hydrophobic interactions (Olsson 2014). 142

Figure 5.2 Examples of anions (Gericke et al. 2012) and cations (Olsson 2014) for ILs with their molecular structures, for cations: (A) imidazolium, (B) pyridinium, (C) tetramethylguanidinium, (D) ammonium, (E) phosphonium. ..................................... 147

Figure 5.3 Proposed dissolution mechanism of cellulose in 1-butyl-3-methylimidazolium chloride (BMIMCl) (Tan 2016). 
Figure 5.4 Constituent ions of ILs used for fiber spinning:11-ethyl-3-methylimidazolium,21butyl-3-methylimidazolium, $\quad 3$ 1-allyl-3-methylimidazolium, 4 1,5diazabicyclo[4.3.0]non-5-enium (Hummel et al. 2015). ................................................ 150

Figure 5.5 Possible materials and technologies generated from cellulose dissolved in ionic liquids (Isik et al. 2014)

Figure 5.6 Common ionic liquid cations used for cellulose dissolution. 1-ethyl-3methylimidazolium acetate is abbreviated EMIMAc (Thunberg 2015).

Figure 5.7 Procedure for the preparation of gel material from a solution of cellulose in BMIMCl

(Kadokawa et al. 2008).................................................................................................... 154

Figure 5.8 Two different approaches to produce cellulose containing ion gels. ......................... 156

Figure 5.9 Dissolution of cellulose with ionic liquids and its application ................................... 156

Figure 5.10 Schematic representation of cellulose-polyamine films with protruding primary

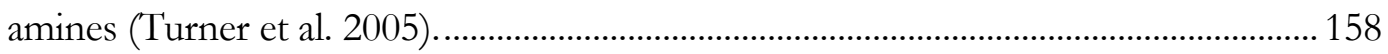

Figure 5.11Two main approaches to obtain cellulose-poly (ionic liquid) composites. $\Delta=$ heat given to the system (Isik et al. 2014).............................................................................. 160

Figure 5.12 Preparation procedure of cellulose-based ionic porous material compatibilized with

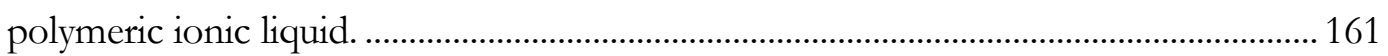

Figure 5.13(a) ligament prototype processing using ionic liquid dissolution, (b) the prototype in tubule form and (c) the microstructure of the prototype walls after partial dissolution (Oksman et al. 2016)... 162

Figure 5.14 various approach for preparing continuous fibers from nanocellulose for different applications. 164

Figure 5.15 (Left) XRD (X-ray diffraction) patterns of cellulose and electrospun fibers from AMIMCl; (Right) SEM (scanning electron microscope) image of fibers produced from $5 \mathrm{wt} \%$ solution; reprinted from (Xu et al. 2008).

Figure 6.1 Synthesis of FITC-Labeled, Folic Acid-Conjugated CNCs (Dong et al. 2014)...... 181

Figure 6.2 Avidin-biotin complexes.

Figure 6.3 Shows how TEMPO can provide carboxyl group on cellulose surface which allow the use of covalent conjugation to Avidin, on that case the whole system can be used for Biotin functionalities. 182

Figure 6.4 Schematic Diagram Ilustrating the preparation of cationic comb-shaped copolymers composed of HPC backbones. Comb-shaped poly-cationic vector can be considered as non-viral gene carriers (Xu et al. 2009).

Figure 6.5 shows the developing of a non-viral gene vector, aminated $\beta$-cyclodextrin (ACD) modified carboxylated magnetic cobalt/nanocellulose composite (ACDC-Co/NCC) for targeting the epithelial cells. 186

Figure 6.6 A layer-by-layer approach has for creating multilayer thin films and microcapsules for controlled drug delivery. 187

Figure 6.7 Chemical structures of IMC and cellulose (an essential componentof NFC), and the possible hydrogen-bonding mode. 189 
Figure 6.8 Schematic illustration of the formation of CNF/IMC composite fibers.

Figure 6.9 Synthesis by aqueous free radical crosslinking polymerization (FRCP), bioconjugation, and dual stimuli-response of biopolymer-based ssBNGs crosslinked with disulfide linkages of POEOMA-g-CMC (Wen and Oh 2014).

Figure 6.10 Preparation routine of single-membrane and double-membrane microsphere hydrogels; optical microscope images of (a) the Sodium alginate (SA)/CCNC singlemembrane microsphere hydrogel and (b) the SA/CCNC-1h double-membrane microsphere hydrogel (Lin et al. 2016).....

Figure 6.11 Proposed mechanism for the formation of semi-interpenetrating polymer network (IPN) hydrogels.

Figure 7.1 Schematic of the life-cycle of cellulose nanocrystals and products made from these nanoparticles (Camarero-Espinosa et al. 2016). 


\section{LIST OF TABLES}

Table 2.1 Some examples of antimicrobial immobilization into cellulose nanofibers (Rezaei et al. 2015)

Table 3.1 Conditions of pulping process, kappa number and screen yield after pulping........... 98

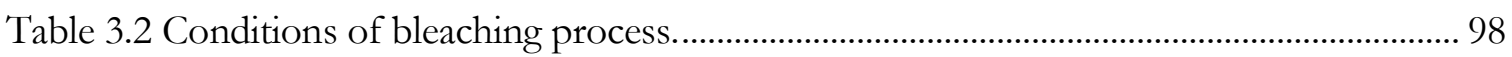

Table 3.3 Chemical characterization of untreated wood (Raw) and bleached pulps (B)........... 98

Table 3.4 Viscosity and degree of polymerization of:Bleached samples and samples after TEMPO oxidation process (TOWP). Note: TOWP: TEMPO-oxidized wood pulp. . 99

Table 3.5 Carboxylic contents of Red oak and Yellow poplar species for bleached and Tempo oxidized samples (TOWP).

Table 3.6 Crystallinities of raw material, TEMPO-oxidized wood pulp (TOWP) and TEMPOoxidized cellulose nanofibers (TNFC).

Table 3.7 Onset degradation temperature (Tonset), maximum thermal degradation temperature (Tmax), maximum weight loss (WLmax) and CY (Char Yield) (\%): (Char residueafter $575 \circ \mathrm{C})$. 100

Table 3.8 Mechanical Properties of Transparent Films of the Oxidized Cellulose Fibrils....... 100

Table 3.9 Copper determination using EDX of hybrid material TNFC-CuNPs based on TNFC

from Red oak and Yellow poplar (three repetitions).......................................................... 100

Table 4.1 Conditions of pulping process.

Table 4.2 Conditions of bleaching process. 129

Table 4.3 Chemical characterization of Kraft pulps (K), Bleached pulps (B), and untreated samples (Ref) of Red Oak: R.O , Yellow poplar: Y.P.

Table 4.4 Viscosity and degree of polymerization of: TOWP R.O, TOWP.P: Samples after TEMPO oxidation, and Bleached samples. Note: TOWP: TEMPO-oxidized wood pulp.

Table 4.5 Carboxylic contents of R.Oak and Y.Poplar species in bleached and Tempo oxidized samples.

Table 4.6 Cristallinities of cellulose fibers at different stages; TNFC: TEMPO nanofibrillated cellulose, TOWP: Tempo-oxidized wood pulp, Raw: Original samples of Red Oak and Yellow Poplar.

Table 4.7 Onset degradation temperature ( $\left.\mathrm{T}_{\text {onset }}\right)$, maximum thermal degradation temperature $\left(\mathrm{T}_{\max }\right)$, maximum weight loss $\left(\mathrm{WL}_{\max }\right)$ and CY (Char Yield) (\%): (Char residueafter 575 $\left.{ }^{\circ} \mathrm{C}\right)$ 131

Table 4.8 Mechanical Properties of Transparent Films of the Oxidized Cellulose Fibrils....... 131

Table 4.9 Copper determination using EDX of hybrid material TNFC-CuNPs based on TNFC from Red oak and Yellow poplar (three repetitions).

Table 7.1 An overview of the published studies focusing on the hazard assessment of nanocellulose(Camarero-Espinosa et al. 2016). 


\section{LIST OF SYMBOLS/abbreviation / NOMENCLATURE}

CMC: Carboxymethyl cellulose

CNC: cellulose nanocrystal

D: Chlorine dioxide

DP: degree of polymerization

$\mathrm{E}$ : Alkaline extraction

EA: Effective alkali

EDX: Energy-dispersive X-ray spectroscopy

FTIR: Fourier transform infrared spectroscopy

HPLC: High-performance liquid chromatography

I002: peak intensity of the main crystalline plane

Iam: peak intensity associated with the amorphous fraction

MFC: MicroFibrillated Cellulose

NFC: Nanofibrillated cellulose

o.d. : Oven dried

P: Peroxide stage

SEM: Scanning electron microscopy

TAPPI/Tappi: Technical association of the pulp and paper industry

TEMPO: 2,2,6,6-tetramethylpiperidine-1-oxyl radical

TEM: Transmission electron microscopy

TGA: Thermogravimetric analyzer

TNFC: TEMPO nanofibrillated cellulose

TOWP: TEMPO oxidized wood pulp

XRD: X-ray diffraction

$\eta$ : viscosity of the fluid 


\section{INTRODUCTION}

Cellulose is the most common polymer on earth and is one of the structural components of plant cell walls (Isogai 2014). Native cellulose is a structural homopolysaccharide consisting of poly- $\beta$ - $(1 \rightarrow 4)$-D-glucosyl molecules that will form crystalline cellulose nanofibrils (nanocellulose) of 3-10 nm in width and several microns in length depending on the origin. Cellulose molecules are fully extended along the longitudinal direction of each cellulose nanofibril, forming numerous and regular intra- and intermolecular hydrogen bonds (Isogai 2014). The polymer chains form nanometer wide fibrils that provide mechanical support in the cell wall of trees and some algae.

Over the last decade the research on the topic "nanocellulose" has increased dramatically and it is expected to continue growing for the next 30 years (Milanez et al. 2013).

Although the nanocellulose term can be used in reference to nanofibrillated cellulose (NFC), microfibrillated cellulose (MFC), cellulose nanocrystals (CNC), cellulose nano whiskers, bacterial cellulose (BC), etc. these terms are not necessary related to the same material in terms of their dimensions. For the rest of this document when we refer to nanocellulose we will specify what the dimensions of the nanomaterial in review are. The particular research presented in this dossier was focused on nanofibrilated cellulose (NFC) which corresponds to cellulosic nanofibers with dimensions less than 50nm wide and lengths in the order of several micrometers. More than $80 \%$ of scientific research papers published on nanocellulose have been focused on NFC (Klemm et al. 2011; Lavoine et al. 2012).

Nanocellulose is renewable, biodegradable, has high strength and stiffness, and possesses a large specific surface area. Some specific characteristics, such as surface charge and dimensions will vary depending on the preparation method and cellulose origin. For specific applications it has been suggested that nanocellulose must be chemically modified (functionalized). 
Oxidation of native celluloses using TEMPO (2,2,6,6-tetramethylpiperidine-1-oxyl radical) as a catalyst is a powerful technique to separate individual cellulosic nanofibrils by the introduction of negatively charged functional groups on their surface. The selective oxidation of the C6-primary hydroxyl groups presented in the cellulosic backbone to carboxylate groups can be achieved by TEMPO/NaBr/NaClO system in water at $\mathrm{pH} 10$ and room temperature. Thus, aqueous TEMPO Nanofibrillated cellulose dispersions can be finally obtained by gentle mechanical disintegration of the oxidized celluloses in water. The resulting cellulose microfibrils originating from wood have uniform widths of $\sim 3-4$ nm and lengths of a few microns.

The introduction of functional groups on the cellulosic backbone have demonstrated capability to bind some molecules of interest such as peptides, antibodies, metals and drugs. The resulting functionalized nanocelluloses will therefore can be used in several fields and novel applications such as packaging, safety, pharmacy, medical, water treatment, cosmetic, etc. (Mahfoudhi and Boufi 2017, Suopajärvi et al. 2014; Suopajärvi et al. 2013, Laitinen et al. 2014, Barazzouk and Daneault 2012).

\section{Motivation.}

Considering that TEMPO nanofibrillated cellulose has a interesting potential in several areas, it is highly motivating to investigate the properties of nanofibrillated obtained from highly available and underutilized hardwood species presented in the Appalachian hardwood forest. Among them, Red oak (Quercus rubra) and Yellow poplar (Liriodendron tulipifera).

\section{Hypothesis}

TEMPO nanofibrillated cellulose from the Appalachian hardwood forest will present similar properties independently on the specie in consideration. In this research two common hardwood species, Red oak (Quercus rubra) and Yellow poplar (Liriodendron tulipifera) were considered in the analysis. 


\section{Main goal}

To evaluate the feasibility to obtain nanofibrillated (NFC) from underutilized Red oak and Yellow poplar and propose potential further applications.

\section{Specific objectives}

The following four specific objectives were defined to address the main goal:

1) Evaluate a TEMPO-mediated oxidation process to produce nanofibrillated cellulose from two highly available hardwoods in the state of West Virginia: Red oak (Quercus rubra) and Yellow poplar (Liriodendron tulipifera).

2) Evaluate specific modifications in the process to produce TEMPO-nanofibrillated cellulose that will increase the functional groups on the surface of the cellulosic backbone. The main goal here was to facilitate the chemical and/or physical incorporation of antimicrobial or drugs components on the cellulosic backbone.

3) In-deep literature review related to the following topics:

3.1 Using ionic liquids as an alternative to produce nanocellulose from woody biomass. 3.2 Nanocellulose-based drug delivery system.

3.3 Safety aspects in the utilization of nanocellulose in food, medical and pharmacy fields.

Research Approach. The following approaches were made to accomplish specific objectives 1) and 2):

Northern Red oak (Quercus rubra)(R.O) and Yellow poplar (Liriodendron tulipifera)(Y.P) samples were collected in 2014 at WVU Research Forest, Preston County, West Virginia, in the form of logging residue. 
Kraft pulp from the oven dried material was produced using a M/K System 6-L laboratory digester.The Kappa numbers of the two species were determined by measuring the consumption of potassium permanganate according to T $236 \mathrm{~cm}-85$ standard method. The percentage of lignin was estimated based on the kappa number.

A five-stage bleaching sequence $\mathrm{D}_{0} \mathrm{EpD}_{1} \mathrm{EpD}_{2}$ was performed using Chlorine dioxide and peroxide reinforced alkaline extraction as the main reagent. The bleaching process was modified for the second objective to introduce higher number of carboxylic groups on the cellulosic backbone surfaces.

TEMPO-oxidation process using the TEMPO/ $\mathrm{NaBr} / \mathrm{NaClO}$ system was used applying different concentration of $\mathrm{NaClO}$.

Mechanical fibrillation was performed by passing the samples twice through an M-110EH30 Microfluidizer. In the first step, the material passed through a $200 \mu \mathrm{m}$ chamber and in the second step the suspension passed through 200- and 87- $\mu \mathrm{m}$ chambers in series.

Modifications in the process to fabricate nanocellulose included modifications in the pulp bleaching process and in the mechanical fibrillation. The mechanical fibrillation was performed by passing the bleached and chemical modified material, around $1 \mathrm{wt} \%$ suspension of TEMPO oxidized cellulose, through an M-110EH-30 Microfluidizer (Microfluidics, Newton, MA). Three passes through a 100 micron diamond orifice at 138 MPa feed pressure was utilized.

Hybrid TNFC-copper nanoparticles were prepared by incorporation of copper nanoparticles on TNFC substrate through chemical reduction (sodium borohydride was used as the reducing agent) of cupric ions. Upon dispersing the hybrid materials into deionized water, they were dried using a preliminary solvent exchange process with ethanol/tert-butanol and then freeze dried. 
Copper determination of TNFC-metallic copper particles was conducted using Energy Dispersive X-ray (EDX) analysis.

TNFC hydrogel films were prepared by filtering TNFC suspensions which were dried afterward. The filtration was performed by first diluting the TNFC suspension to $0.2 \%$ solid by weight with reverse-osmosis-treated water. Following the dilution, the suspensions were filtered using a 142-mm Millipore ultrafiltration system (Millipore Corporation, Billerica, MA, USA) under 0.55 MPa of air pressure. Omnipore TM filter membranes with a pore size of $0.1 \mu \mathrm{m}$ (JVWP14225, JV, Millipore Corporation, USA) were supported on filter paper in the ultrafiltration system. The wet films (hydrogels) were peeled from the membrane, processed, stacked, and placed between an assembly of waxy coated papers, absorbent blotter paper, and two metal caul plates. The assembly was air-dried at room temperature for $24 \mathrm{~h}$ and then oven-dried at $60^{\circ} \mathrm{C}$ for $8 \mathrm{~h}$ under a load of approximately $250 \mathrm{~N}$. The blotter and filter papers were replaced several times over the first $24 \mathrm{~h}$ to minimize wrinkling of the films.

The tensile strength of TNFC films were tested according to ASTM D638-10 standard using an Instron 5865 universal material testing apparatus (Instron Engineering Corporation, MA, USA) with a $500 \mathrm{~N}$ load cell.

Chemical analysis was done which included Klason lignin, acid soluble lignin, hemicellulose and cellulose determination. High performance anion exchange chromatography (Dionex ICS-3000 system) been used for carbohydrate determination. Lignin was determined based on the amount of sulfuric acid-insoluble Klason lignin, in accordance with the standard of the Technical association of pulp and paper industry (TAPPI) TAPPI standards-T222 om 88.

Viscosity of the resulting TEMPO oxidized cellulose was measured according to TAPPI Standard Method T230 om-99 and has been compared with the untreated samples. Degree of polymerization was measured indirectly as a function of viscosity. 
Determination of carboxyl content of the samples before and after the TEMPO-mediated oxidation process was performed according to T 237 om-88with some reagent concentration modifications.The pulps were converted to the acid form by soaking them in $0.01 \mathrm{M} \mathrm{HCl}$ and then washing with deionized water. This acidified pulp was reacted with a standardized $0.1 \mathrm{M}$ NaHCO3 with $0.25 \mathrm{M} \mathrm{NaCl}$ solution. The amount of residual $\mathrm{NaHCO} 3$ was determined by titration with $0.1 \mathrm{M} \mathrm{HCl}$, to a methyl red endpoint. The carboxyl contentwas calculated in miliequivalents (meq) per $100 \mathrm{~g}$ of oven-dry pulp and then converted to mmol/g.

Fourier transform infra-red spectroscopy (FTIR)were recorded for the analysis of the functional groups of the raw material, bleached pulps, TEMPO-oxidized pulps and the final TEMPO-nanofibrillated cellulose (TNFC)samples using a Perkin-Elmer Spectrum 2000 FTIR spectrometer.

The crystalline structure and hence the chemical composition of the raw material, TEMPOoxidized pulps, and the final TEMPO nanofibrillated cellulose (TNFC) samples and copper nanoparticles within the films were investigated under X-ray diffraction (XRD). The analysis was performed on a X-Ray diffractometer (PANalyticalX'Pert Pro XRD) in the range of $10^{\circ}$ to $40^{\circ} 2 \theta$. Cu-kal $8047.2 \mathrm{eV}$ source and a maximum X-Ray power of $45 \mathrm{kV}$ and $40 \mathrm{~mA}$ were used.

Thermogravimetric (TGA) and derivative thermogravimetric (DTG) analyses were performed for the raw material and the final TEMPO-nanofibrillated cellulose (TNFC)to study the hermal properties using a TA Q50 thermogravimetric analyzer (Delaware, USA)to compare the thermal degradation characteristics of the untreated and chemo-mechanical treated R.O and Y.P.

The morphology of the samples was characterized by scanning electron microscopy (SEM) using a Hitachi S-4700 FE-SEM; the samples were previously coated with gold-palladium using a sputtering process to provide adequate conductivity to evaluate the effect of mechanical fibrillation on the samples morphology. 
Transmission electron microscope (TEM) of TNFC samples of R.O and Y.P were used as their original undiluted suspension form. Samples were imaged using Philips CM-100 TEM

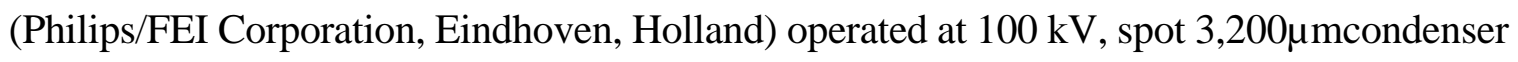

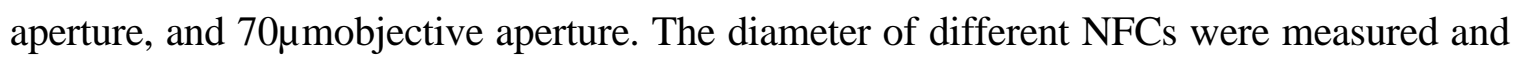
calculated from TEM images using the ImageJ Manipulation Program

The surface morphology of cellulose/copper nanoparticles was observed under scanning electron microscopy (SEM).

Dissertation organization. This thesis includes 7 chapters.

In chapter 1 is presented a background on cellulose, different types of nanocellulose, nanocellulose preparation methods, surface modification strategies, and an introduction about TEMPO- oxidation as a surface modification method.

Chapter 2 is a literature review which describes in detail current and potential application of nanocellulose.

Chapter 3 presents the process to fabricate TEMPO nanofibrillated cellulose from underutilized Red oak (Quercus rubra) and Yellow poplar (Liriodendron tulipifera) and describes a full characterization of the intermediate and final products. In addition, in this manuscript is presented the feasibility of using the NFC as template for copper nanoparticles as antimicrobial material. The information presented in this chapter was published in the Journal of Nanomaterials, Hassanzadeh et al. 2017.

In Chapter 4 is presented the production of nanofibrillated cellulose from Red oak (Quercus rubra) and Yellow poplar (Liriodendron tulipifera) using a modified approach. The main goal was to increase the number of carboxylic groups on the nanocellulosic material. The increase of carboxylic groups on the nanocellulosic material was meant to improve its capability to attach copper nanoparticles as antimicrobial material. 
Chapter $\mathbf{5}$ presents a literature review regarding to the utilization of ionic liquids to dissolve, and perform chemical modification on cellulosic raw materials for nanocellulosebiomaterials production.

In Chapter $\mathbf{6}$ is presented a literature review related to nanocellulose-based drug delivery systems and finally,

In chapter 7, safety aspects in the utilization of cellulosic based materials in food and medical/pharmacy fields is presented.

The following publications are, so far, the result of the research performed:

1) Hassanzadeh M., Sabo R., Rudie A., Reiner R., Gleisner R. and G. S. Oporto. 2017. Nanofibrillated Cellulose from Appalachian Hardwoods Logging Residues as Template for Antimicrobial Copper. Journal of nanomaterials. Volume 2017, Article ID 2102987. https://doi.org/10.1155/2017/2102987

2) Hassanzadeh M., Oporto G.S., Sabo R. and A. Rudi. 2016. Nanofibrillated cellulose from Appalachian Hardwoods Red Oak (Quercus rubra) and Yellow Poplar (Liriodendron tulipifera) as template for copper nanoparticles. In Proceedings of the 59th International Convention of Society of Wood Science and Technology, March 6-10, 2016, Curitiba, Brazil.

\section{References}

Barazzouk S, Daneault C (2012) Amino acid and peptide immobilization on oxidized nanocellulose: spectroscopic characterization. Nanomaterials 2:187-205

Isogai A (2014) Cellulose. Encyclopedia of Polymeric Nanomaterials, 329-339.Springer, Berlin.

Klemm D, Kramer F, Moritz S, Lindström T, Ankerfors M, Gray D, Dorris A (2011) Nanocelluloses: A new family of nature-based materials. Angewandte Chemie International Edition 50:5438-5466

Laitinen O, Kemppainen K, Ämmälä A, Sirviö JA, Liimatainen H, Niinimäki J (2014) Use of chemically modified nanocelluloses in flotation of hematite and quartz. Industrial \& Engineering Chemistry Research 53:20092-20098 
Lavoine N, Desloges I, Dufresne A, Bras J (2012) Microfibrillated cellulose-Its barrier properties and applications in cellulosic materials: A review. Carbohydrate polymers 90:735-764

Mahfoudhi N, Boufi S (2017) Nanocellulose as a novel nanostructured adsorbent for environmental remediation: a review. Cellulose:1-27

Milanez DH, Amaral RMd, Faria LILd, Gregolin JAR (2013) Assessing nanocellulose developments using science and technology indicators. Materials Research 16:635641

Suopajärvi T, Koivuranta E, Liimatainen H, Niinimäki J (2014) Flocculation of municipal wastewaters with anionic nanocelluloses: Influence of nanocellulose characteristics on floc morphology and strength. Journal of Environmental Chemical Engineering 2:2005-2012

Suopajärvi T, Liimatainen H, Hormi O, Niinimäki J (2013) Coagulation-flocculation treatment of municipal wastewater based on anionized nanocelluloses. Chemical Engineering Journal 231:59-67 


\section{CHAPTER 1: LITERATURE REVIEW NANOCELLULOSE}

\subsection{Cellulose}

In 1838, the French chemist Anselme Payen reported that he had isolated a fibrous carbohydrate material by subjecting various plant tissues to acid and ammonia treatments followed by water extraction. That material was named cellulose (Klemm et al. 2005). Today it is known that cellulose is a polymer with the primary role of providing structural support in the cell walls of land plants, bushes and trees as well as some algae (Dufresne 2013). It is also produced by fungi, some bacteria and amoebas as well as tunicates (a sea creature). Cellulose is the most abundant polymer on earth and the annual worldwide production has been estimated to be $1.5 \times 10^{12}$ tons. It has traditionally been used for e.g. construction and as an energy source in the form of wood, as well as for producing paper and textiles (Klemm et al. 2011; Moon et al. 2011).

Cellulose is a linear homopolymer composed of D-glucopyranose units linked through $\beta$ (14) glycosidic bonds, where each unit is twisted $180^{\circ}$ with respect to the neighboring units. Therefore the repeat unit is considered to be cellobiose, which consists of two Dglucopyranose units. Furthermore, 30 - 40 cellulose polymer chains aggregate into microfibrils (also referred to as elementary fibrils) that are $3-5 \mathrm{~nm}$ wide. Within each of these microfibrils, there are regions, where the cellulose chains are arranged in highly ordered (crystalline) structures, and regions that are disordered (Figure 1.1) (Moon et al. 2011). 


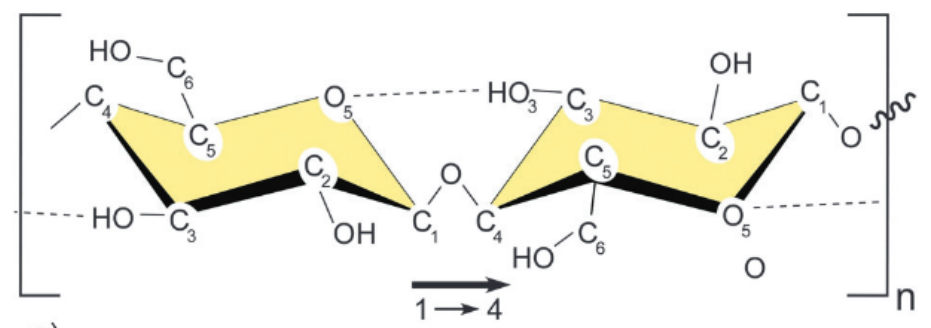

a)

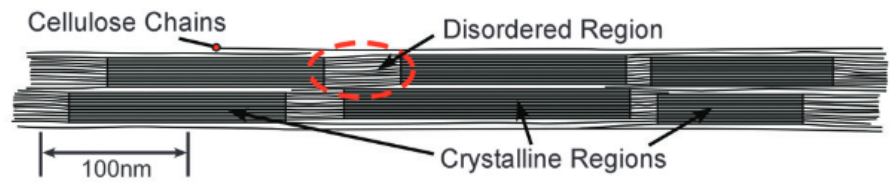

b)

c)

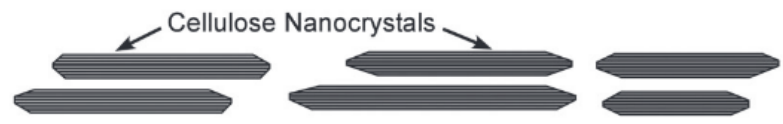

Figure 1.1 Schematic representation of (a) single cellulose chain repeat unit, showing the directionality of the 1-4 linkage and intrachain hydrogen bonding (dotted line), (b) idealized cellulose microfibril showing one of the suggested configurations of the crystalline and amorphous regions, and (c) cellulose nanocrystals after acid hydrolysis dissolved the disordered regions (Moon et al. 2011).

As it is schematically illustrated in

Figure 1.2, cellulosic material together with hemicelluloses and lignin, form the plant cell wall (fiber) (Eichhorn et al. 2010; Isogai et al. 2011).

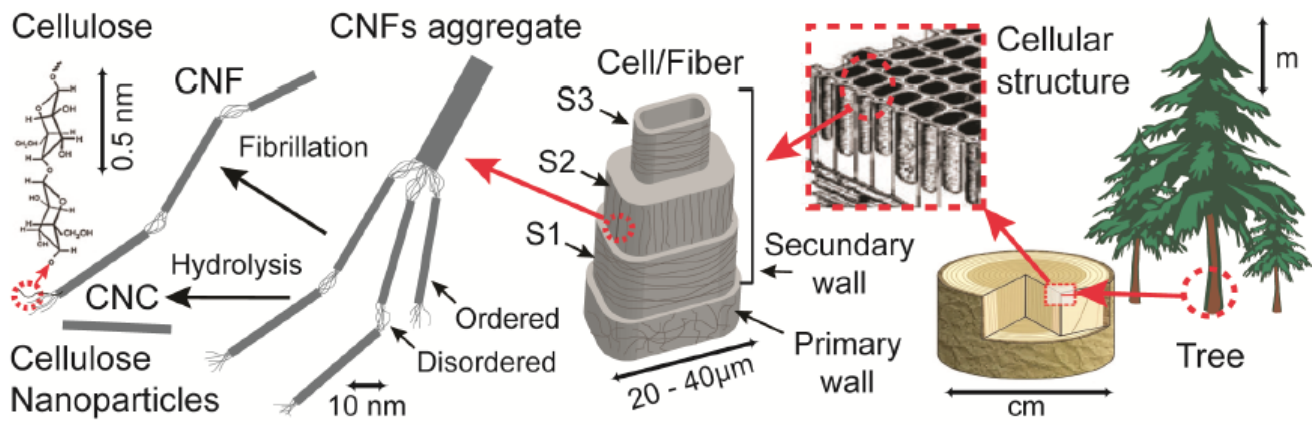

Figure 1.2 Schematic illustration of the hierarchical structure of cellulose in wood and cellulose nanoparticles isolation (Postek et al. 2010) 
The cellulosic hydroxyl and oxygen atoms facilitate intra- and interchain hydrogen bonding. Intrachain bonding stabilizes the polymer chain, making the structure rather stiff and linear. The interchain hydrogen bonding promotes stacking and ordering of the chains, resulting in crystalline structures, as well as in less ordered chain structures. Due to the large number of hydroxyl groups and oxygen atoms on each cellobiose unit, there are several different crystal structures, or polymorphs. They differ in their hydrogen bonding pattern and, hence, stability. The crystalline form found in nature is called cellulose I and can, through different treatments, form cellulose II, III and IV (Dufresne 2013).

Cellulose II, for example, can be formed by two processes: regeneration, which involves solubilization and recrystallization; and mercerization, which involves strong alkali treatment. Cellulose I can be further divided into cellulose I $\alpha$ and I $\beta$, which differ in their hydrogen bonding pattern. Cellulose I $\alpha$ (monoclinic) and I $\beta$ (triclinic) coexist in different proportions depending on cellulose source. For example, cellulose from algae is dominated by $I \alpha$, while cellulose from trees is domi-nated by I $\beta$. I $\beta$ is the more stable form and I $\alpha$ can be converted, at least partly, to $\mathrm{I} \beta$ by annealing at $260-280^{\circ} \mathrm{C}$ in e.g. $0.1 \mathrm{M} \mathrm{NaOH}$ or organic solvents (Debzi et al. 1991; Dufresne 2013).

During biosynthesis, the cellulose chains are assembled into semicrystal-line elementary fibrils that contain crystalline regions (cellulose I $\alpha$ and I $\beta$ ) as well asnon-crystalline regions. In wood, these fibrils are 3-5 $\mathrm{nm}$ in width and they aggregate to form microfibrils, or macrofibrils, which are up to $60 \mathrm{~nm}$ in width and several micrometers long. However, the distinction be-tween the different fibrils in terms of size, and the organization and nature of the non-crystalline regions is still debated but it is generally assumed that the fibrils are composed of alternating crystalline and non-crystalline (amorphous or paracrystalline) regions.

The key point, however, is that the smallest semicrystalline fibrils aggregate into larger fibril bundles which are embedded in a matrix material, forming a hierarchically ordered natural composite material in the cell wall (Figure 1.3), where the fibrils constitute the main 
reinforcing component. The matrix material consists of mainly hemicellulose (a group of amorphous and branched polysaccharides) glued to the cellulosic material through lignin polymer (Knoshaug et al. 2013).

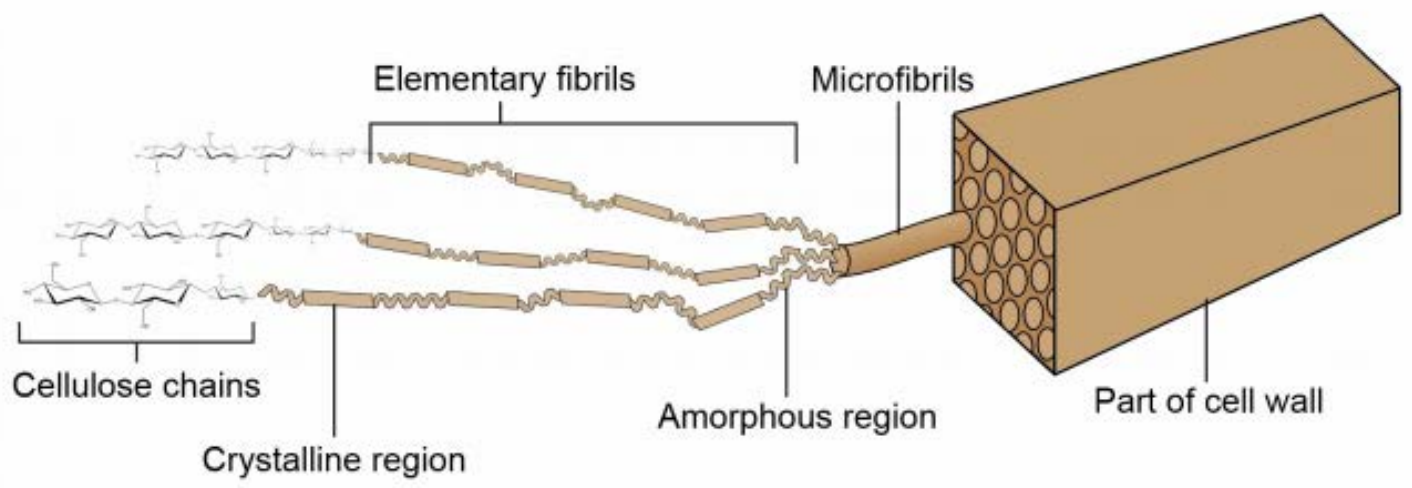

Figure 1.3 Simplified illustration of the hierarchical organization of cellulose in the wood cell wall (Lavoine et al. 2012).

The size and form of the individual crystalline regions (crystallites), as well as the fibrils, varies depending on the cellulose source and stem from differences in the biosynthesis processes. For example, the crystallites in wood are 3-5 nm in width whereas the crystallites in algae, e.g. Cladophora andValonia, are significantly larger, 20 nm (Moon et al. 2011).

\subsection{Nanocellulose}

Methods to produce nanocellulose combine chemical, mechanical and enzymatic treatments to disintegrate wood fibers (Araki et al. 1998; Nakagaito and Yano 2005). Based on the treatment fibrils with a diameter of 5-30 $\mathrm{nm}$ and length in micrometers, or rod-like crystals with a diameter of 5-20 nm and length of tens to hundreds of nanometers to micrometers can be obtained. The nanocellulose (fibril/crystal) dimensions strongly depends on the treatment type and the source, but they are always nanosized in at least one dimension (Dufresne 2013). Microfibrillated cellulose (MFC) is the name first adopted for the fibril material from woodsource (Herrick et al. 1983) which has been referred later to nanofibrillated cellulose (NFC) or cellulose nanofibrils (CNF) (Isogai 2013; Klemm et al. 2011). Cellulose nanowhiskers or simply whiskers have been used for the nanosized crystals. Currently the 
terms nanocrystalline cellulose (NCC) or cellulose nanocrystals (CNC) are more commonly used. (Azizi Samir et al. 2005; de Souza Lima and Borsali 2004; Fleming et al. 2001).

\subsection{Nanofibrillated cellulose (NFC)}

Because of its large availability and well established industry, the most common source material of CNFs is wood. Nanofibrillated cellulose (NFC) can be prepared from wood based pul pmaterial by simultaneous enzymatic and mechanical treatment (Pääkkö et al. 2007) or just mechanical treatment (Nakagaito and Yano 2004). The mechanical and chemical treatment ofwood chips removes the non-cellulosic components and to break the cellular structure of wood, individualized wood cells are obtained. These cells are known in the pulp and paper industry as fibers. The fibers are typically a few millimeters long and 20-40 $\mu \mathrm{m}$ wide. Since pulp prepared by chemical methods has higher cellulose content, this pulp is preferred as a starting material for the preparation of NFCs.

Due to its nanoscale dimensions, NFC has extraordinary properties. The long and entangled fibrils have high aspect ratio and large surface area providing vast amounts of reactive hydroxyls groups on their surface (Pääkkö et al. 2007; Zimmermann et al. 2010).Upon drying NFC forms hierarchical nanoporous aerogels or strong films depending on the drying process. In water, NFC forms a gel, which strength is dependent on the fibril concentration (Pääkkö et al. 2007; Pääkkö et al. 2008). The mechanical properties of NFC paper films have been reported to be better than ordinary paper and the reinforcing ability in composite materials has also been shown to be superior compared to pulp fibers (Nakagaito and Yano 2005). This is caused by the larger surface area of fibrils exhibiting extensive hydrogen bonding compared to macroscopic fibers. Moreover, these films have good gas barrier properties which can be of benefit in many coating and barrier technologies (Fukuzumi et al. 2008). The aerogels have been shown to have very different properties compared to traditional aerogels due to the long and entangled fibrils forming a hierarchical nanoporous structure (Pääkkö et al. 2008). They open up new application areas for the use of aerogels in general. Due to its biocompatibility NFC is also a promising material for biotechnological and pharmaceutical applications (Dufresne 2013; Lin and Dufresne 2014). 


\subsection{Cellulose nanocrystals (CNC)}

Cellulose nanocrystals (CNC, Figure 1.4 (b)) can be obtained from different sources by acid hydrolysis using a concentrated mineral acid (Elazzouzi-Hafraoui et al. 2007; Habibi et al. 2010). A typical production process involves acid hydrolysis, washing, centrifugation, dialysis, and sonication to form a suspension followed by drying (Habibi et al. 2010). The main process for the preparation of CNCs is based on strong acid hydrolysis under strictly controlled conditions of concentration, temperature, agitation, and time. The mineral acid breaks the $\beta-1,4$ glycosidic bonds in cellulose. During hydrolysis, the amorphous regions are attacked, leaving the crystalline regions intact (Yue et al. 2012) and yielding a dispersion of rod-like crystalline cellulose sticks. These nanosized rods range in size depending on the source they are obtained from and by the reaction conditions but usually are 5 to $20 \mathrm{~nm}$ in width and 0.1 to $\sim 1 \mu \mathrm{m}$ in length (Beck-Candanedo et al. 2005; de Souza Lima and Borsali 2004). Microbial hydrolysis has also been utilized to produce CNCs. This microbial hydrolysis is eco-friendly and does not require any surface modification (Satyamurthy et al. 2011). The charge and colloidal behavior of CNCs depends on the acid used for the production. Sulfuric and hydrochloric acids are the most commonly used, but phosphoric and hydrobromic acids have also been used (Lee et al. 2012).

CNC has smaller aspect ratio than NFC and in solution does not form as strong gel as NFC due to the lack of extensive percolation of the network (Liu et al. 2011a; Pääkkö et al. 2007). Yet the rods are mechanically extremely strong. Experimentally, the Young's modulus of a single crystal of cellulose I is 134 GPa and the strength in the GPa range (Sakurada et al. 1962).

The mineral acid used in the hydrolysis of cellulose material determines whether the resulting CNC will carry charge on their surface or not (Fleming et al. 2001). If sulfuric acid (50-70\% $\mathrm{w} / \mathrm{v}$ ), temperature of $20-70^{\circ} \mathrm{C}$, rate of $500 \mathrm{rpm}$, and time of $0.5-6 \mathrm{~h}$ are employed, esterification also occurs forming “cellulose sulfate,” resulting in a negatively charged surface on the cellulose crystallites. Longer hydrolysis time and higher temperature generate shorter nanocrystals with higher surface charge, high crystallinity ( $80 \%)$, and narrower poly- dispersity (Brito et al. 2012; Ioelovich 2012). 
By hydrochloric acid hydrolysis the surface of the crystals will be almost neutral and display almost solely hydroxyl groups. These CNC are usually referred to as HCl-CNC (Araki et al. 1998). The sulfate-CNCs are readily dispersed in water due to their charged surface. They form stable colloidal dispersions in water but can be aggregated with increasingionic strength (Araki et al. 1998; Fleming et al. 2001).
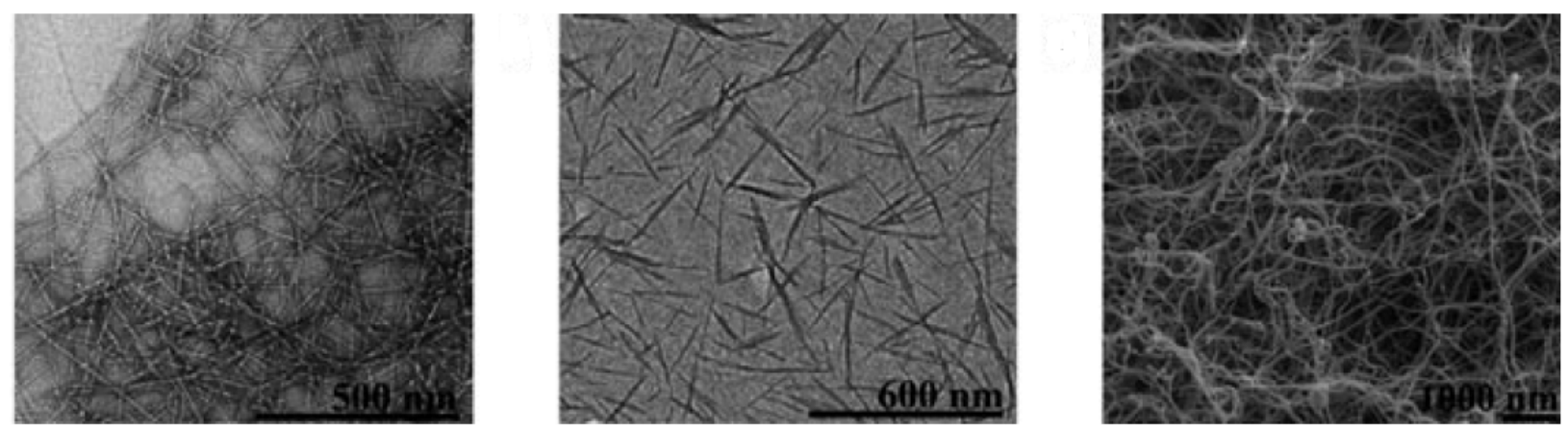

Figure 1.4 Transmission electron micrographs of a) MFC and b) NCC; c) scanning electron micrograph of BNC (Klemm et al. 2011).

\subsection{Bacterial nanocellulose (BNC)}

Bacterial nanocellulose (BNC) is a highly biocompatible material with unique properties produced by several species of bacteria in aqueous culture media containing carbon and nitrogen sources during a time period of days (Habibi 2014). The most important of these is Acetobacter xylinum, now renamed Gluconacetobacter xylinus, discovered in 1886 by A. J. Brown. These bacteria are Gram-negative, aerobic bacterium forming BNC(s) in static culture on the surface of the culture medium as a white, gelatinous material in form of a hydrogel. These bacteria are highly ubiquitous; They are found wherever the fermentation of sugars and plant carbohydrates takes place; for example, on the surfaces of rotting fruits and in unpasteurized or unsterilized juice, beer, and wine (Gama et al. 2012).

\subsection{Nanocellulose preparation methods}

Nanocellulose can be prepared through many different approaches, which can be classified into two general top-down and bottom-up categories (Hentze 2010). The top-down approaches obtains nanocellulose by extracting cellulose particles from various sources at 
nanoscale and can be further divided into three subcategories: mechanical, chemical, and mechanical-chemical. The bottom-up approaches assemble cellulose nanostructures either from the solution state of cellulose molecules or from the biosynthesis process (Hentze 2010; Klemm et al. 2011). Figure 1.5 shows different approaches of nanocellulose production. The most important ways for nanocellulose fabrication are described below.

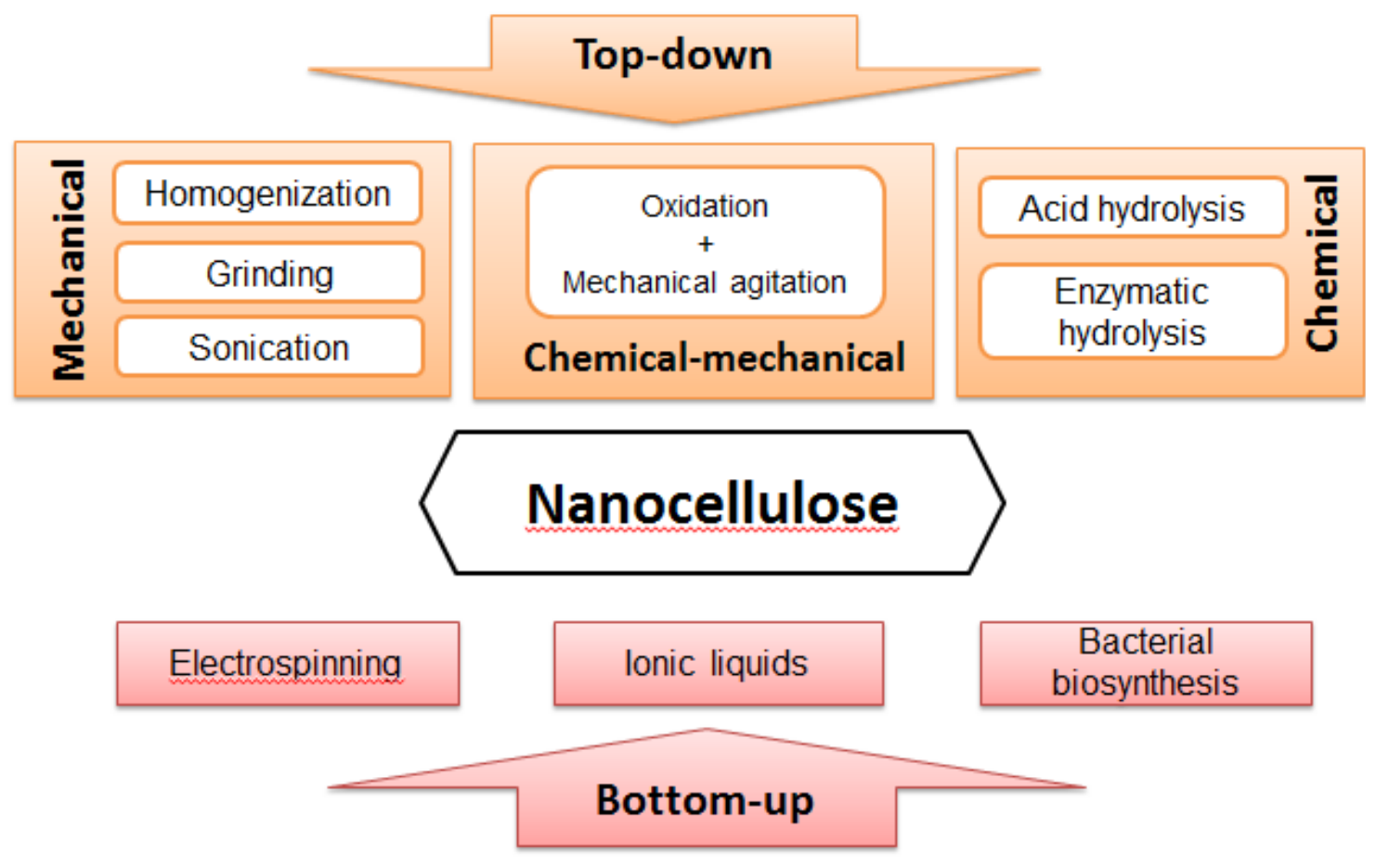

Figure 1.5 Classification of nanocellulose fabrication approaches (adapted from Li, 2012).

\subsubsection{Top-down approaches to produce nanocellulose}

As it comes from the name, the top-down approaches typically start from raw materials like wood chips, pulps, cotton litters. From these sources mechanical, chemical, or combined chemical-mechanical methods would be used to liberate nanocelluloses from their native recalcitrant structures (i.e.: cell wall or large fiber bundles). For high purity raw materials like cotton linter, this means to release individual microfibrils from the large fiber bundles. Whereas for the raw materials existed in the natural composite form like wood chips, the top-down approach means to separate cellulose from its lignin- 
hemicellulose matrix first then isolate individualized nanocellulose particles from large cellulose fiber bundles (Li 2012).

\subsubsection{Mechanical methods to produce nanofibrillated cellulose}

\section{Homogenization:}

Turbak et al. 1983, described the method for the first time. The homogenization method disintegrated wood pulp fibers into NFCs by passing several times a pulp suspension through a homogenizer at high pressure with intensive homogenization treatments with controlled pass numbers and high pressure through a small orifice (55 MPa or $8000 \mathrm{psi}$ ). The predominant end product has a net-like structure, with a diameter ranging from 25-100 nm in dry state. Due to the large increase in surface area, MFC has a dramatically increased water retention rate of more than $400 \%$ (compared to $50-90 \%$ of wood pulps). Large energy consumption (25,000 kWh/t) is the major limitation of commercial success (Klemm et al. 2011; Spence et al. 2011).

Wet grinding:

Abe et al. 2007 introduced the wet grinding method to process lignin containing plant fibers in order to yield uniform nanocellulose fibrils with dimension of 12-16 nm. Before the grinding process, samples were pretreated in order to remove lignin and hemicellulose components from the cell wall matrix. The delignified samples were then passed through the grinder at $1500 \mathrm{rpm}$ in slurry condition (1 wt\%) to isolate the nanocellulose fibrils.

\section{Sonication:}

Wuhrmann et al. 1946, reported the first attempt to produce MFC with a mechanical agitation method, where plant fibers from ramie, hemp, and cotton were subjected to intensive sonication treatment. From the process, fine fibrils in 6-7 nm width were yielded as a function of the mechanical agitation intensity.

\subsubsection{Chemical methods to produce nanocellulose crystals}

Acid hydrolysis 
As mentioned previously, acid hydrolysis is a well-known process for removing accessible regions from native cellulose (amorphous) and produce nanocellulose crystals (NCC). Different cellulosic materials (pulps, plant-based cellulose, bacterial cellulose, tunicate cellulose, etc.) have been treated with concentrated mineral acid (most commonly sulfuric acid or hydrochloric acid) under elevated temperatures withdifferent time periods.The end products are rod-like particles with lateral dimensions ranging from 3-30 nm and hundreds of nanometers in length, depending on the processing conditions as well as the starting materials. The NCC produced by acid hydrolysis usually forms liquid crystalline structures, and exhibit chiral nematic phaseproperties in certain concentration range (Bondeson et al. 2006; Revol et al. 1992).

\subsubsection{Chemical-mechanical methods to produce nanofibrillated cellulose}

In order to reduce the energy consumption during the production of nanocellulose, a combination of chemical and mechanical treatments has been adopted by many research groups around the globe and the most up-to-date research progress has been summarized by Isogai and coworkers (Isogai et al. 2011). Different chemical pretreatments includes oxidation, acid hydrolysis, and enzymatic hydrolysis, while mechanical treatments include sonication, homogenization, blending, etc. TEMPO oxidation, chloroacetic acid etherification,enzymatic hydrolysis, and carboxymethylation are examples of most common chemical methods in which the cellulose would be oxidize or hydrolyze, and then mild to intensive mechanical treatments would be applied to liberate carboxylated microfibrillated cellulose (MFC) or Nanofibrillated cellulose from the raw materials.The final product NFC will have lateral dimensions of single digit nanometers (or even subnanometers) and hundreds of nanometer to several microns in length (Bondeson et al. 2006; Eyholzer et al. 2010; Li and Renneckar 2009; Pääkkö et al. 2007; Saito et al. 2006a; Saito el al. 2006b; Wågberg et al. 1987).

\subsubsection{Bottom-up approaches to produce nanocellulose}

Contrary to the top-down approaches, the bottom-up approaches produce nanocellulose through assembling from its molecular level building blocks not from the raw materials. Some examples include bacterial cellulose synthesis (BC) and Electrospinning process. In the case of bacterial 
cellulose synthesis, nanocellulose is polymerized from UDP-glucose via the bacterial metabolic activity. For electrospinning, the nanocellulosic material is regenerated into solid fibrous form from the solution state (Li 2012).

\subsubsection{Bacterial biosytnthesis}

Bacterial nanocellulose (BNC, bacterial cellulose, microbial cellulose, biocellulose) is produced by certain bacteria's biosynthesis process which converting low-molecularweight sugars or other carbon sources via uridine diphosphate glucose to cellulose molecules. The cellulose fibrils released by the bacteria combined to form ribbons and finally a 3D nanofiber network. BNC formed as described has the same molecular formula as plant cellulose but is fundamentally different because of its nanofiber architecture. After simple purification, BNC contains no impurities and no functional groups other than hydroxy groups (Klemm et al. 2011)

\subsubsection{Electrospinning}

An electrospinning process uses a polymer solution (or polymer melt) to spin continuous fibers under an electrical field under the high voltage. The solution forms because of the repulsion of charges at the surface of the drop. Once the charge repulsion overcomes the surface tension of the polymer, a fine jet is then ejected towards the collector to form the nanocellulose. Electrospinning can produce nanocellulose in non-woven fiber mat form or oriented fiber strands dependent on the configuration of the collector; fiber diameter ranges from single digit nanometers to microns (Rodriguez et al. 2011).

\subsection{Modification of nanocellulose surface properties}

Nanofibrillated cellulose displays two main drawbacks, which are associated with its intrinsic physical properties. The first one is the high number of hydroxyl groups, which lead to strong hydrogen interactions between two nanofibrils and to the gel-like structure once produced. Due to the hydrophilic nature of cellulose, NFC cannot be uniformly dispersed in most non-polar polymer media and its suspension is a gel-like structure at very low concentration and the NFC forms films or aggregates once dried. The second drawback is the high hydrophilicity of this material, which limits its uses in several applications such as 
in paper coating (increase of dewatering effect) or composites (tendency to form agglomerates in petro-chemical polymers). The most feasible solution to this is chemical surface modification to reduce the number of hydroxyl interactions and also to increase the compatibility with several matrices.

Consequently, NFC modification is of interest in order to open-up new applications in a veriety of areas. Compatibility with a wider variety of matrices used in coating colors or in extrusion can be also attempted. NFC surface modification can also help to introduce new functionalities and to produce “active” NFC (Missoum et al. 2013). All chemical functionalizations have been mainly conducted to (1) introduce stable negative or positive electrostatic charges on the surface of cellulose nanocrystals (CNs) to obtain better dispersion (CNs obtained after sulfuric acid hydrolysis introduce labile sulfate moieties that are readily removed under mild alkaline conditions) and (2) tune the surface energy characteristics of CNs to improve compatibility, especially when used in conjunction with nonpolar or hydrophobic matrices in nanocomposites. The main challenge for the chemical functionalization of CNs is to conduct the process in such a way that it only changes the

surface of CNs, while preserving the original morphology to avoid any polymorphic conversion and to maintain the integrity of the crystal (Habibi et al. 2010).

\subsubsection{Surface modification strategies}

The properties of a substrate surface can be modified in several ways to facilitate a certain application by so called functionalization, including introduction of surface charges, attachment of specific surface groups as well as deposition of coatings on top of the substrate. The reactive hydroxyl groups, amply present on the nanocellulose surface, are often used as a starting point for thefunctionalization of nanocellulose (Eichhorn et al. 2010; Tingaut et al. 2012).

Identification of the optimum surface modification is very important for NFC quality and can be done by the following ways:

A. Verifying the treatment regime which should not damage the cellulosis structure. 
B. Optimizing qualities such as impact resistance, modulus of elasticity and strength to breakage by controlling the bonding degree of structural elements and matrix in composite (Khalil et al. 2014).

New functionalities can be introduced already during the nanocellulose preparation as mentioned above, where surface charges are introduced to further the disintegration process or to obtain stable nanocellulose dispersions. Surface charges may also be introduced after the nanocellulose has been extracted. One way to introduce surface charges is through etherification of the hydroxyl groups with monochloroacetic sodium salts, resulting in anionic carboxymethyl groups on the fibril surfaces (Figure 1.6a).Cationic surface charges can also be introduced through etherification, using epoxypropyltrimethylammonium chloride (EPTMAC), yielding quaternary ammonium (hydroxypropyltrimethylammonium) groups on the fibril surfaces (Figure 1.6 (b)).

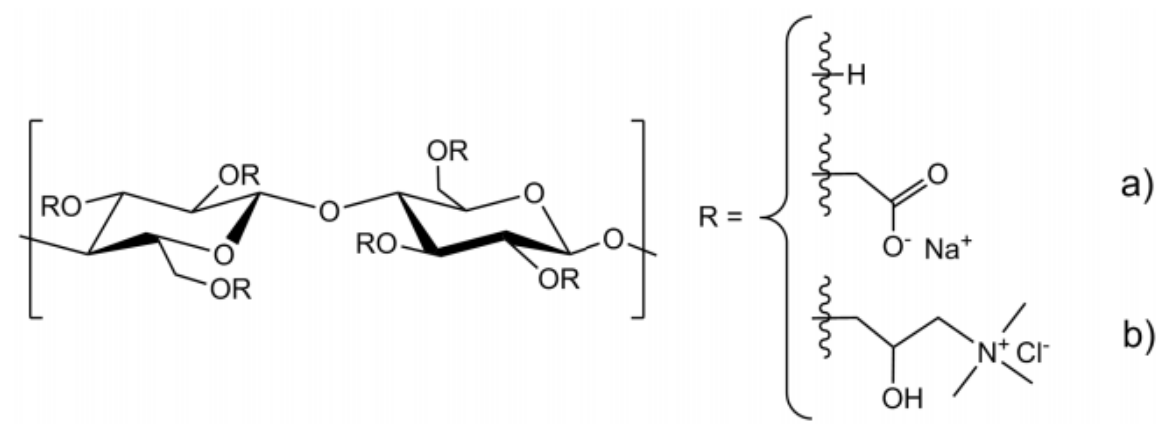

Figure 1.6 Cellulose functionalized with anionic carboxymethyl groups (a) and quaternary ammonium groups (b) (Habibi 2014).

Other types of functionalities than charged groups can also be introduced. For example, hydrophobic properties can be introduced through acetylation and silylation (Figure 1.7). Another example is polymer grafting, where a range of different polymers with various properties have been attached to the surface, either by grafting to or from the surface.Functionalization via non-covalent interactions, where surfactants or polyelectrolytes are adsorbed onto the surface, can also be applied, often with the purpose of improving the nanocellulosedispersibility or to control the assembly of individual fibrils into layered structures. 


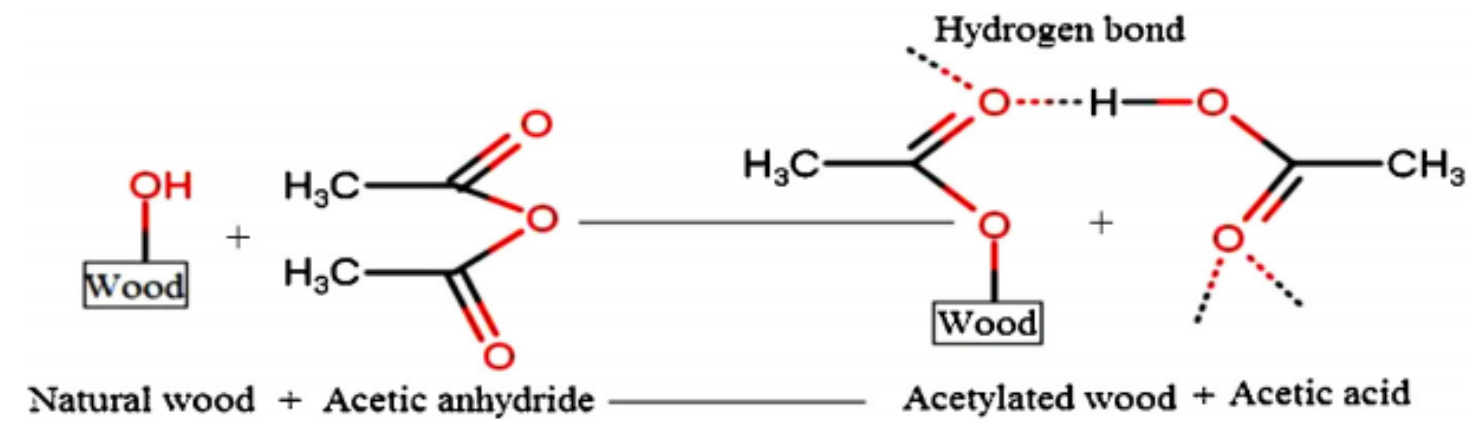

Figure 1.7 A scheme of wood acetylation mechanism (Khalil et al. 2014).

Another way of functionalizing nanocellulose is to coat the fibrils with other materials, thereby forming composite materials. Since the emergence a few years ago, nanocellulose aerogels have been employed as substrates to produce new functional materials with high porosities and large surface areas. For example $\mathrm{TiO} 2$ and silanes have been deposited on aerogels to produce hydrophobic sponges for separation of oil from water. Different ways for chelating (Functionalization) including TEMPO has been presented on following schematic picture (Figure 1.8):

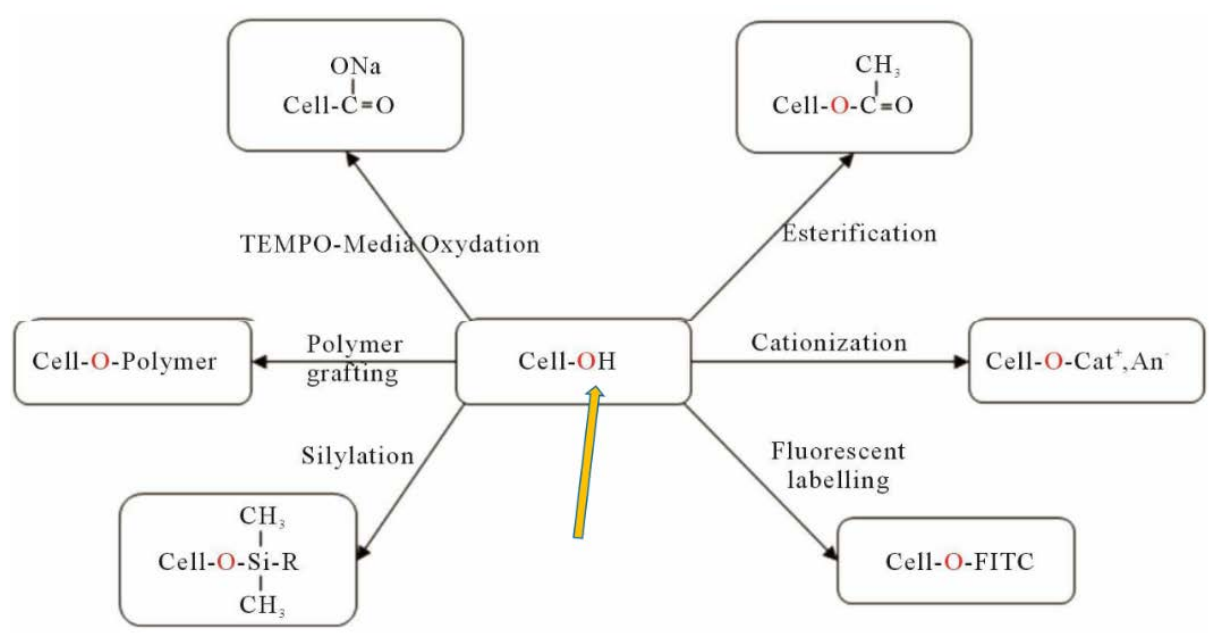

Figure 1.8 Examples of chemical modification of nanocellulose. 


\subsubsection{TEMPO - mediated oxidation}

TEMPO (2,2,6,6-tetramethylpiperidine-1-oxyl) - mediated oxidation is another method of introducing anionic surface charges.

2,2,6,6-Tetramethylpiperidine-1-oxyl (TEMPO) oxidation is a kind of pretreatments that facilitate isolation of nanofibers. This method selectively introduces carboxyl (acidic) groups at the C6 of glucose unit (Iwamoto et al. 2011). In TEMPO oxidation process, an additional catalyst $(\mathrm{NaBr})$ and primary oxidant $(\mathrm{NaClO})$ are applied at $\mathrm{pH}$ 9-11 (Saito et al. 2009).Mechanism of TEMPO oxidization of cellulose is illustrated in Figure 1.9 (Isogai and Kato 1998).

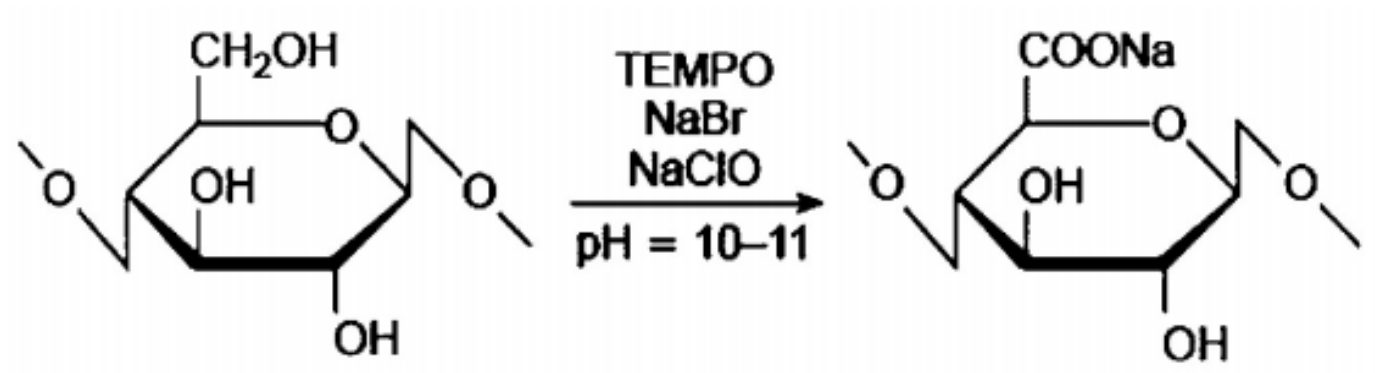

Cellulose

Figure 1.9 TEMPO oxidization of cellulose (Isogai and Kato 1998).

This oxidation reaction, which is highly discriminative of primary hydroxyl groups, is also "green” and simple to implement. It involves the application of a stable nitroxyl radical, the 2,2,6,6 tetramethylpiperidine-1-oxyl (TEMPO), in the presence of $\mathrm{NaBr}$ and $\mathrm{NaOCl}$ (see Figure 1.9).

TEMPO is a radical and a weak oxidizing agent, but can be oxidized to an oxoammoniumcation, which is a much stronger oxidizing agent. Thus, in order to oxidize the TEMPO radical, additional oxidants, such as $\mathrm{NaClO}$ and $\mathrm{NaBrO}$, are used to continuously regenerate the oxoammonium cation form of TEMPO. The oxoammonium ions then selectively oxidize the cellulose C6 hydroxyls to carboxylates via an aldehyde intermediate. Catalytic oxidation by the $\mathrm{NaClO} / \mathrm{NaBr} / \mathrm{TEMPO}$ system was shown in Figure 
1.10. NaClO plays a role as primary oxidant to formNaBrO, which in turn oxidizes TEMPO (a) to form the nitrosoniumcompound (b). The nitrosonium compound (b) oxidizes theprimary hydroxyl to carboxylate via aldehyde structure in water atpH 10 , forming $\mathrm{N}$ hydroxylamine (c). The nitrosonium compound (b) is regenerated from (c) via TEMPO in the catalytic system, and only inexpensive $\mathrm{NaClO}$ and $\mathrm{NaOH}$ are consumed (Iwamoto et al. 2010).

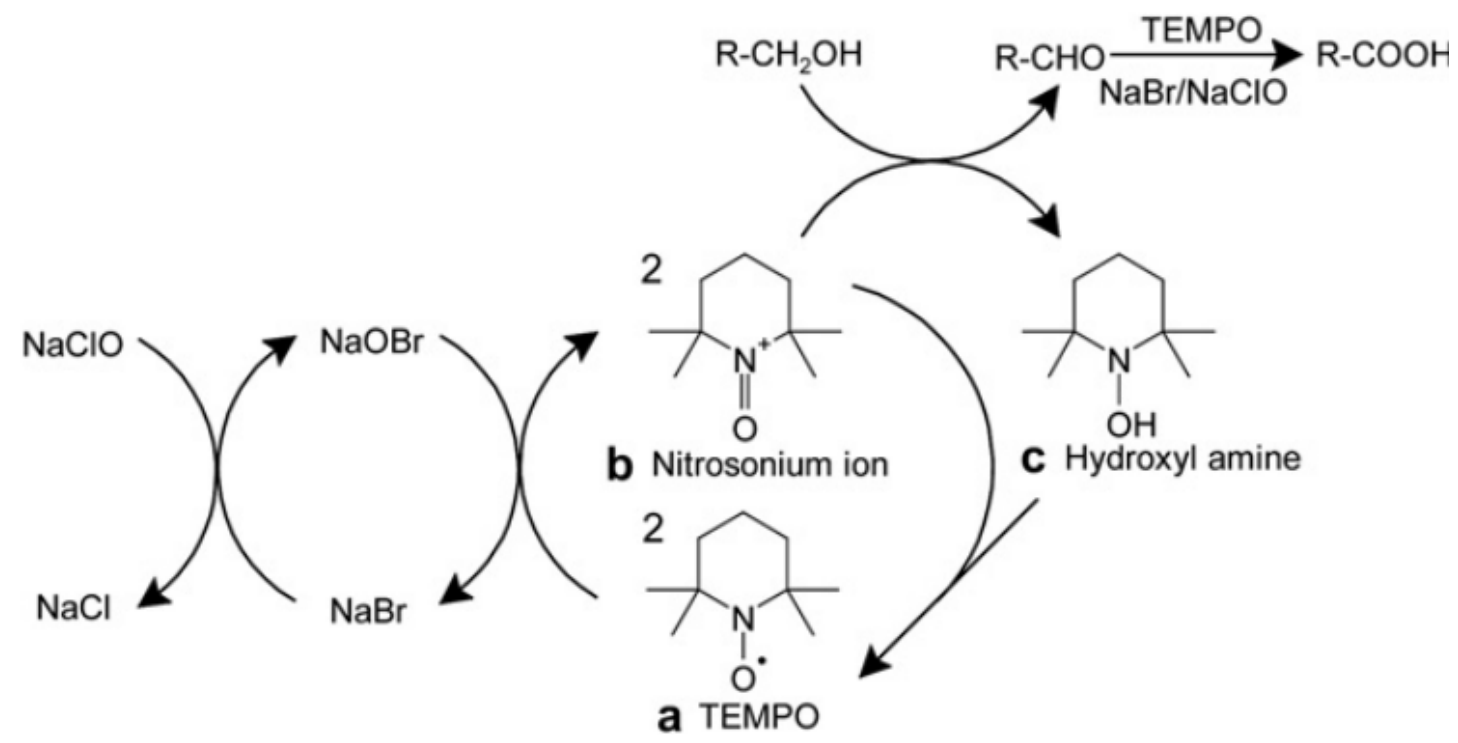

Figure 1.10 Reaction scheme of TEMPO-mediated oxidation of primary hydroxyls (Iwamoto et al. 2010)

The use of this technique has been the subject of a number of reports since it was first introduced by De Nooy et al., who showed that only the hydroxymethyl groups of polysaccharides were oxidized, while the secondary hydroxyls remained unaffected. In fact, TEMPO-mediated oxidation of CNs involves a topologically confined reaction sequence, and as a consequence of the 2-fold screw axis of the cellulose chain, only half of the accessible hydroxymethyl groups are available to react, whereas the other half are buried within the crystalline particle (Figure 1.11)(Habibi 2014). 

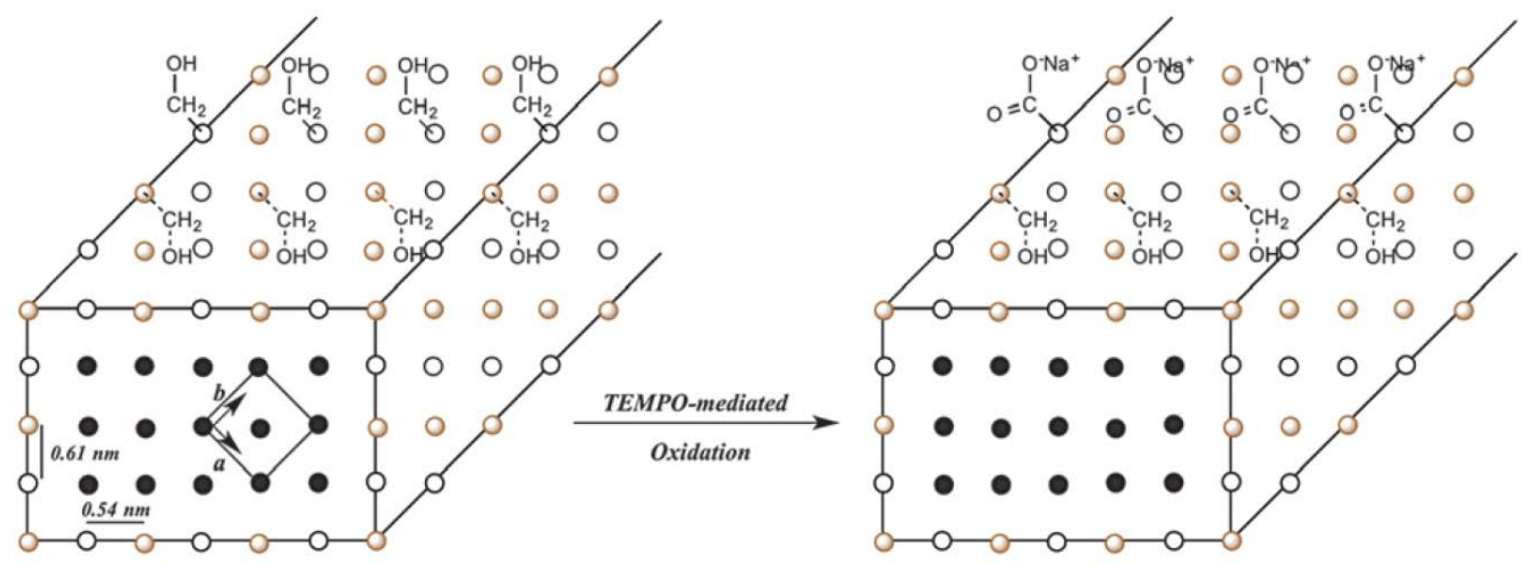

Figure 1.11 Schematic representation of a CNC cross section illustrating the selective TEMPO-mediated oxidation of primary hydroxyl groups at the surface of the crystal (Habibi et al, 2014)

In the case of NFCs, TEMPO-mediatedoxidationismostlyused as a cost-effective chemical treatment prior to the mechanical processing in order to facilitate the individualization of the fibers. Indeed, after impairing their structural potential and softening their rigid structure by breaking the strong intra-fiber hydrogen bond networks, the TEMPO-oxidized cellulose fibers can be converted very easily, utilizing mechanical shearing discussed above (high speed blending, ultrasonication, high mechanical shearing,etc.), into highly crystalline individual nanofibers. This method has been employed for a variety of cellulosic fibers. With the most common TEMPO derivative, the optimal conditions reported are a $\mathrm{pH}$ of 10 and temperature ranging between 0 and 101C. These operating conditions usually yield cellulose nanofibres having 3-20 nm in width and a few microns in length depending on the cellulosic raw material used. It should be noted that TEMPO-mediated oxidation was conducted as an intermediate step to promote the grafting of (macro) molecule entities through amidation. It has also been used in order to introduce stable negative electrostatic charges on the surface of CNCs to obtain better dispersion comparable to that obtained after sulfuric acid hydrolysis. Indeed, TEMPO-mediated oxidation of CNCs, obtained from $\mathrm{HCl}$ hydrolysis of cellulose fibers, was first reported by Arakiet al.1998. The authors demonstrated that after TEMPOmediated oxidation, the CNCs maintained their initial morphological integrity and formed a homogeneous suspension when dispersed in water. The basis for the latter observation was the presence of carboxyl groups that imparted negative charges at the CNC surface and thus 
induced electrostatic stabilization. When dispersed in water, TEMPO-oxidized or carboxylated CNC suspensions display birefringence patterns and do not flocculate or sediment owing to the polyanionic character imparted by the negative charges on the CNC surfaces. Such interesting behavior is only obtained through sulfuric acid hydrolysis and often ascribed to the presence of sulfate ester groups that are negatively charged. In addition to promoting the dispersion of NFCs and CNCs in water, the incorporation of sodium carboxylate groups at their surfaces seems to be beneficial for their dispersion in aprotic organic solvents such as DMAc, DMF, DMI, and NMP particularly after converting the sodium carboxylate groups to free carboxyl groups (Habibi et al. 2010; Isogai and Kato 1998).

Even if the washing step of these tempo-treated fibers is still the main drawback for their industrialization, they have several advantages. Indeed, compared to the energy consumption of repeated cycles of a high pressure homogenizer (700-1400 MJ/kg), TEMPO-mediated oxidation pre-treatment drastically decreases the consumption to values less than $7 \mathrm{MJ} / \mathrm{kg}$. The nanofibrils within the fibers separate from each other more easily due to the repulsive forces of the ionized carboxylate groups, which overwhelm the hydrogen bonds holding them together. TEMPO oxidation pre-treatment is usually followed by a mechanical treatment, which can be performed using the devices mentioned before but also with a simpler system like a mixer. However, it is worth keeping in mind that, one key step in this process is the "post-separation" of smaller NFC and bigger NFC by centrifugation. Only the supernatant (smaller NFC) is usually considered as TOCN. The ensuing TOCN material is shorter and thinner than with enzymatic pretreatment which presents other properties completely different from those obtained by the enzymatic way (Missoum et al. 2013).

\subsection{Potential Applications of TEMPO-oxidized Celluloses}

Because carboxylate and aldehyde groups are present only on cellulose I crystal surfaces and in disordered regions, the TEMPO-mediated oxidation can be regarded as one of the most efficient surface modification methods for native cellulose fibers under aqueous conditions. Araki et al. 1998 employed TEMPO-mediated oxidation to prepare carboxyl functionalized microcrystalline celluloses for subsequent conversion to stabilized colloidal suspensions. by 
grafting amine-terminated polyethylene glycol chains (PEG) onto carboxylic sites of the TEMPO-oxidized microcrystals steric stabilization was readily accomplished. The grafted samples produced stable suspensions in the presence of high concentration electrolyte (2M $\mathrm{NaCl}$ ). The grafted microcrystals could also form stable birefringent suspensions as well as liquid crystalline chiral nematic structures in organic solvents (Araki et al. 2001).

Successful grafting of non-polar groups onto TEMPO-oxidized celluloses has also been reported by other researchers (Follain et al. 2008; Lasseuguette 2008).

It has been found that tensile strength of cellulose sheets soaked in water, i.e. wet strength, was remarkably improved, when TEMPO-oxidized native cellulose fibers were used for sheet making (Saito and Isogai 2006b).

Wet strength is one of the significant functionalities for cellulosic products such as washand-wear shirt, filter paper, tissue paper, paper towel and others. Generally, wet strength resins are added to cellulose slurries to produce wet strength cellulose sheets, where some water-resistant interactions are formed between cellulose fibers through the resin molecules during the sheet-making process. In the case of wet strength development of the TEMPOoxidized cellulose sheets, aldehyde groups formed as intermediate structures by the TEMPOmediated oxidation may contribute to the wet strength development of the sheets. These surface aldehyde groups forms hemiacetal linkages with cellulose hydroxyl groups at the inter-fiber bonds, resulting in the clear wet strength development of the sheets prepared.The TEMPO-mediated oxidation of native cellulose fibers, therefore, allows for introduction ofsurface aldehyde groups into native cellulose fibers, which form inter-fiber hemiacetal linkages in sheet (Figure 1.12) (Saito and Isogai 2006b).

Alila et al. used carboxyl functionalized celluloses (synthesized via TEMPO-mediated oxidation) as substrates in the co-adsorption of cationic surfactants and some organic hydrophobic compounds (Alila et al. 2007).

Surfactant aggregation onto charged solid substrates finds applications in a variety of fields including soil remediation, chemical sensors, synthetic membranes, cosmetic formulation, 
and immobilization of organic pollutants. Resultsfrom their studies showed that the adsorbed amounts of both surfactants and organic solutes strongly depended on the degree of TEMPOoxidation i.e. the concentration of carboxylate groups on the oxidized fiber surfaces.For most organic solutes, adsorbed amounts increased 4 to 6 fold when cellulose fibers were preoxidized with TEMPO (Alila et al. 2005; Aloulou et al. 2004).

In another study transparent, conductive and printable nanocomposites were successfully prepared by mixing single-walled carbon nanotubes (CNTs) with 2,2,6,6tetramethylpiperidine-1-oxyl (TEMPO)-oxidized cellulose nanofibrils (TOCNs) with abundant sodium carboxyl groups on the crystalline nanocellulose surfaces. The surfaceanionic cellulose nanofibrils had reinforcing and nanodispersing effects on the CNTs both in water used as the dispersed medium and in the dried composite film, providing highly conductive and printable nanocomposites with a small amount of CNTs. TOCNs are therefore expected as an effective flexible matrix that can be used as an alternative to conventional polymers for various electrical materials, when nanocomposited with CNTs and also graphene (Koga et al. 2013). 


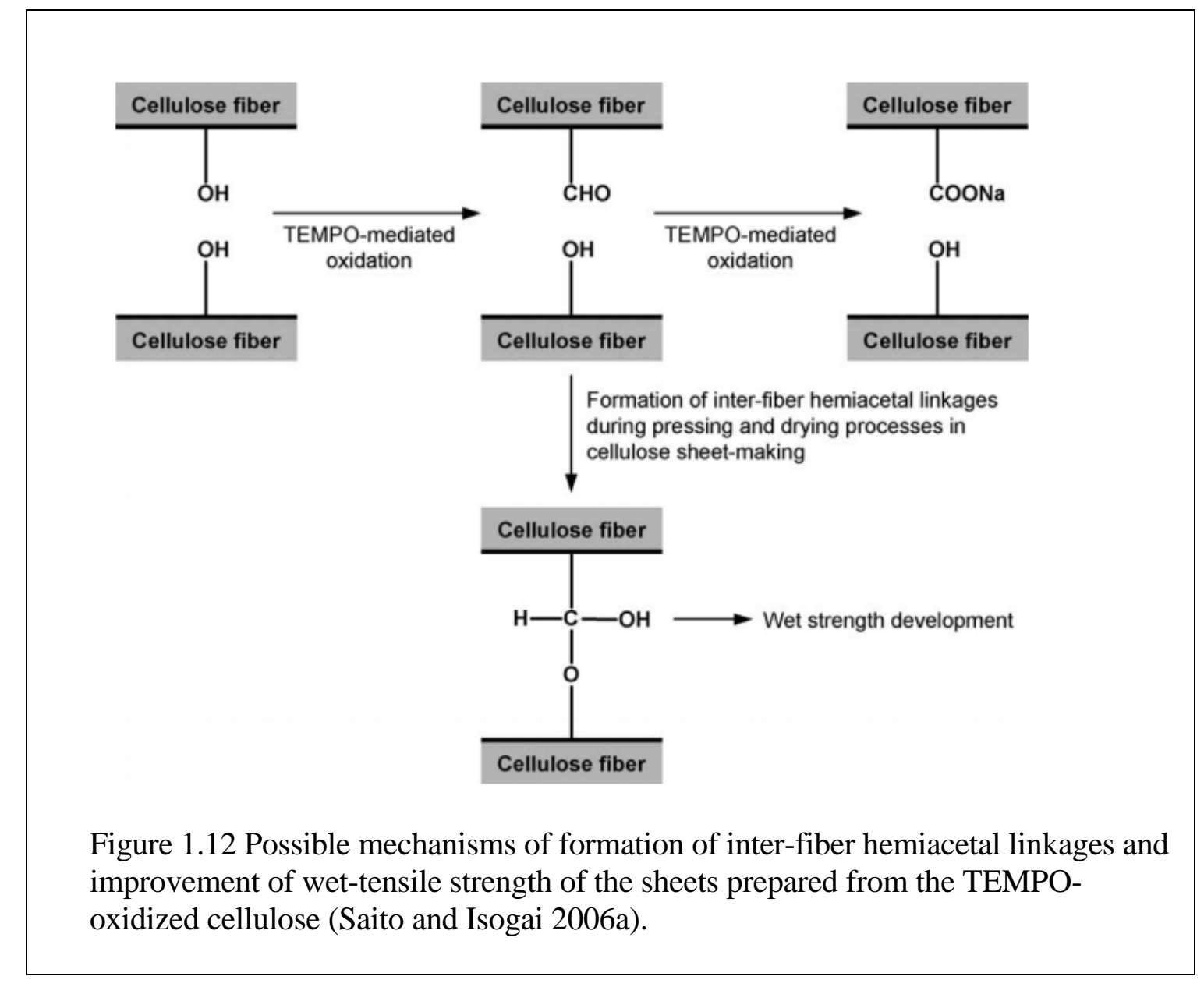

Mechanically robust cellulose nanofibril (CNF) aerogels with ultra-low density $\left(8 \mathrm{mg} \mathrm{cm}^{-3}\right)$, superior porosity (99.5\%), super water absorbency (104 g g-1 water/dried mass), high crystallinity (68.5\%) as well as exceptional wet resilience and water activated shape recovery were facilely fabricated for the first time by ice-crystal templated self-assembly of TEMPO oxidized CNFs via cyclic freezing-thawing (Jiang and Hsieh 2014).

In addition, careful adjustment of the $\mathrm{pH}$ and solvent evaporation in TOCN dispersions produce a wide range of artificial bulk materials with outstanding properties. Examples include unprecedentedly stiff freestanding hydrogels with a water content of $99.9 \%$ and ultralow density and tough aerogels with large surface areas. These materials are expected to be further developed as robust frameworks for polymer nanocomposites or high-capacity supports for catalysts, and the other functional materials. On the other hand, porous TOCN 
networks, similar in appearance to spider webs, have been prepared by direct air drying of TOCN/surfactant dispersions using support materials containing micrometer- or submicrometer-sized pores. These network structures can be categorized as nanoporous materials. The conversion systems used to form bulk materials from TOCN/water dispersions containing self-aligned TOCN elements are summarized in Figure 1.13 (Isogai 2013).

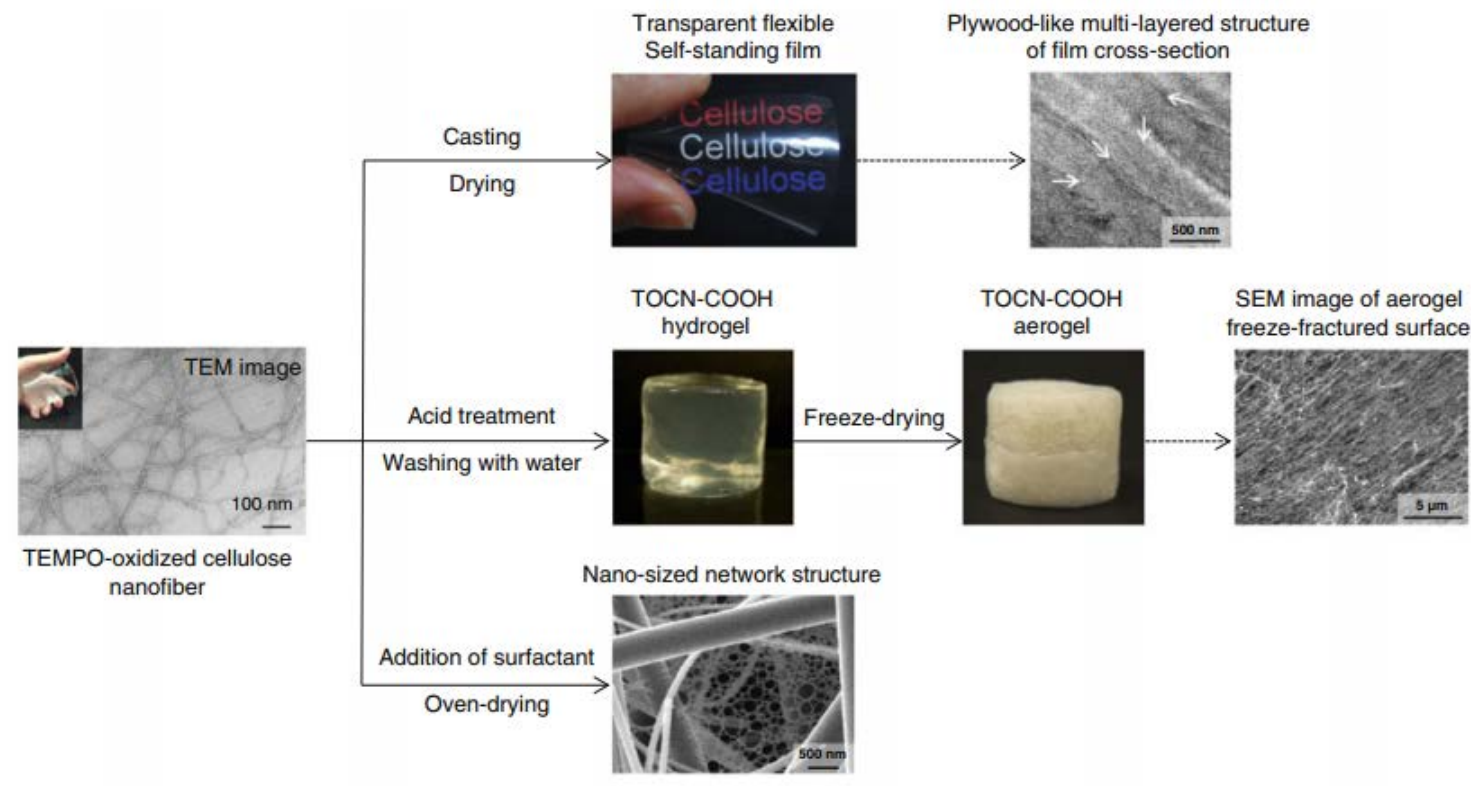

Figure 1.13 Conversion from TOCN/water dispersion to diverse bulk materials (Isogai 2013).

Another interesting application is the use of cellulose nanostructure as a templateand stabilizer for metallic nanoparticles with a goal of use as an antimicrobial nanocomposite for packaging materials.Copper-Carboxymethyl Cellulose Composite Material for packaging purposes has been reported by Zhong et al. 2013 and Zhong et al. 2015a.

A range of different nanomaterials such as metal NPs ( $\mathrm{Au}, \mathrm{Ag}, \mathrm{Pd}, \mathrm{Ni}, \mathrm{TiO}_{2}, \mathrm{CuO}$ ), mineral nanomaterials $\left(\mathrm{CaCO}_{3}, \mathrm{SiO}_{2}\right.$, montmorillonite), and carbonaceous nanomaterials (carbon nanotube, graphene) have been incorporated into or onto nanocellulose substrates which can open a route for different application e.g. for food packaging system if they have antimicrobial properties (Wei et al, 2014).Between them Copper is widely known as a broad- 
spectrum biocide which is less toxic for human being and less expensive and is one of 26 essential elements occurs in our body (Palza 2015).

Antimicrobial bacterial cellulose-silver nanoparticles composite membranes have been obtained by "in situ" preparation of Ag nanoparticles from hydrolytic decomposition of silver nitrate solution using triethanolamine as reducing and complexing agent (Barud et al. 2011).

\subsection{References}

Abe, K., Iwamoto, S. \&Yano, H. (2007). Obtaining cellulose nanofibers with a uniform width of $15 \mathrm{~nm}$ from wood. Biomacromolecules 8(10): 3276-3278.

Alila, S., Aloulou, F., Beneventi, D. \&Boufi, S. (2007). Self-aggregation of cationic surfactants onto oxidized cellulose fibers and coadsorption of organic compounds. Langmuir 23(7): 3723-3731.

Alila, S., Boufi, S., Belgacem, M. N. \&Beneventi, D. (2005). Adsorption of a cationic surfactant onto cellulosic fibers I. Surface charge effects. Langmuir 21(18): 81068113.

Aloulou, F., Boufi, S., Belgacem, N. \&Gandini, A. (2004). Adsorption of cationic surfactants and subsequent adsolubilization of organic compounds onto cellulose fibers. Colloid and Polymer Science 283(3): 344-350.

Araki, J., Wada, M. \&Kuga, S. (2001). Steric stabilization of a cellulose microcrystal suspension by poly (ethylene glycol) grafting. Langmuir 17(1): 21-27.

Araki, J., Wada, M., Kuga, S. \&Okano, T. (1998). Flow properties of microcrystalline cellulose suspension prepared by acid treatment of native cellulose. Colloids and Surfaces A: Physicochemical and Engineering Aspects 142(1): 75-82.

Araki, J., Wada, M., Kuga, S. \&Okano, T. (1998). Flow properties of microcrystalline cellulose suspension prepared by acid treatment of native cellulose. Colloids and Surfaces A: Physicochemical and Engineering Aspects 142(1): 75-82.

Azizi Samir, M. A. S., Alloin, F. \&Dufresne, A. (2005). Review of recent research into cellulosic whiskers, their properties and their application in nanocomposite field. Biomacromolecules 6(2): 612-626.

Barud, H. S., Regiani, T., Marques, R. F., Lustri, W. R., Messaddeq, Y. \&Ribeiro, S. J. (2011). Antimicrobial bacterial cellulose-silver nanoparticles composite membranes. Journal of Nanomaterials 2011: 10.

Beck-Candanedo, S., Roman, M. \&Gray, D. G. (2005). Effect of reaction conditions on the properties and behavior of wood cellulose nanocrystal suspensions. Biomacromolecules 6(2): 1048-1054.

Bondeson, D., Mathew, A. \&Oksman, K. (2006). Optimization of the isolation of nanocrystals from microcrystalline cellulose by acid hydrolysis. Cellulose 13(2): 171-180. 
Brito, B. S., Pereira, F. V., Putaux, J.-L. \&Jean, B. (2012). Preparation, morphology and structure of cellulose nanocrystals from bamboo fibers. Cellulose 19(5): 15271536.

de Souza Lima, M. M. \&Borsali, R. (2004). Rodlike cellulose microcrystals: structure, properties, and applications. Macromolecular Rapid Communications 25(7): 771787.

Debzi, E., Chanzy, H., Sugiyama, J., Takeley P. Excoffier, G. (1991). The I $\alpha \rightarrow$ I $\beta$ transformation of highly crystalline cellulose by annealing by various mediums. Macromolecules 24(26): 6816-6822.

Dufresne, A. (2013). Nanocellulose: a new ageless bionanomaterial. Materials Today 16(6): 220-227.

Eichhorn, S. J., Dufresne, A., Aranguren, M., Marcovich, N., Capadona, J., Rowan, S., Weder, C., Thielemans, W., Roman, M. \&Renneckar, S. (2010). Review: current international research into cellulose nanofibres and nanocomposites. Journal of Materials Science 45(1): 1.

Elazzouzi-Hafraoui, S., Nishiyama, Y., Putaux, J.-L., Heux, L., Dubreuil, F. \&Rochas, C. (2007). The shape and size distribution of crystalline nanoparticles prepared by acid hydrolysis of native cellulose. Biomacromolecules 9(1): 57-65.

Eyholzer, C., Bordeanu, N., Lopez-Suevos, F., Rentsch, D., Zimmermann, T. \&Oksman, K. (2010). Preparation and characterization of water-redispersible nanofibrillated cellulose in powder form. Cellulose 17(1): 19-30.

Fleming, K., Gray, D. G. \&Matthews, S. (2001). Cellulose crystallites. Chemistry-A European Journal 7(9): 1831-1836.

Follain, N., Montanari, S., Jeacomine, I., Gambarelli, S. \&Vignon, M. R. (2008). Coupling of amines with polyglucuronic acid: Evidence for amide bond formation. Carbohydrate polymers 74(3): 333-343.

Fukuzumi, H., Saito, T., Iwata, T., Kumamoto, Y. \&Isogai, A. (2008). Transparent and high gas barrier films of cellulose nanofibers prepared by TEMPO-mediated oxidation. Biomacromolecules 10(1): 162-165.

Gama, M., Gatenholm, P. \&Klemm, D. (2012). Bacterial nanocellulose: a sophisticated multifunctional material. CRC Press.

Habibi, Y. (2014). Key advances in the chemical modification of nanocelluloses. Chemical Society Reviews 43(5): 1519-1542.

Habibi, Y., Lucia, L. A. \&Rojas, O. J. (2010). Cellulose nanocrystals: chemistry, selfassembly, and applications. Chemical reviews 110(6): 3479-3500.

Herrick, F. W., Casebier, R. L., Hamilton, J. K. \&Sandberg, K. R. (1983).Microfibrillated cellulose: morphology and accessibility. In J. Appl. Polym. Sci.: Appl. Polym. Symp.;(United States), Vol. 37: ITT Rayonier Inc., Shelton, WA.

Ioelovich, M. (2012). Optimal conditions for isolation of nanocrystalline cellulose particles. Nanoscience and Nanotechnology 2(2): 9-13.

Isogai, A. (2013). Wood nanocelluloses: fundamentals and applications as new bio-based nanomaterials. Journal of wood science 59(6): 449-459.

Isogai, A. \&Kato, Y. (1998). Preparation of polyuronic acid from cellulose by TEMPOmediated oxidation. Cellulose 5(3): 153-164. 
Isogai, A., Saito, T. \&Fukuzumi, H. (2011). TEMPO-oxidized cellulose nanofibers. Nanoscale 3(1): 71-85.

Iwamoto, S., Kai, W., Isogai, T., Saito, T., Isogai, A. \&Iwata, T. (2010). Comparison study of TEMPO-analogous compounds on oxidation efficiency of wood cellulose for preparation of cellulose nanofibrils. Polymer Degradation and Stability 95(8): 1394-1398.

Jiang, F. \&Hsieh, Y.-L. (2014). Super water absorbing and shape memory nanocellulose aerogels from TEMPO-oxidized cellulose nanofibrils via cyclic freezing-thawing. Journal of Materials Chemistry A 2(2): 350-359.

Khalil, H. A., Davoudpour, Y., Islam, M. N., Mustapha, A., Sudesh, K., Dungani, R. \&Jawaid, M. (2014). Production and modification of nanofibrillated cellulose using various mechanical processes: a review. Carbohydrate Polymers 99: 649665.

Klemm D, Heublein B, Fink HP, Bohn A (2005) Cellulose: fascinating biopolymer and sustainable raw material Angewandte Chemie International Edition 44:3358-3393

Klemm, D., Kramer, F., Moritz, S., Lindström, T., Ankerfors, M., Gray, D. \&Dorris, A. (2011). Nanocelluloses: A new family of nature-based materials. Angewandte Chemie International Edition 50(24): 5438-5466.

Knoshaug, E. P., Shi, B., Shannon, T. G., Mleziva, M. M. \&Pienkos, P. T. (2013). The potential of photosynthetic aquatic species as sources of useful cellulose fibers- $\mathrm{a}$ review. Journal of applied phycology 25(4): 1123-1134.

Koga, H., Saito, T., Kitaoka, T., Nogi, M., Suganuma, K. \&Isogai, A. (2013). Transparent, conductive, and printable composites consisting of TEMPO-oxidized nanocellulose and carbon nanotube. Biomacromolecules 14(4): 1160-1165.

Lasseuguette, E. (2008). Grafting onto microfibrils of native cellulose. Cellulose 15(4): 571-580.

Lavoine, N., Desloges, I., Dufresne, A. \&Bras, J. (2012). Microfibrillated cellulose-Its barrier properties and applications in cellulosic materials: A review. Carbohydrate Polymers 90(2): 735-764.

Lee, K.-Y., Tammelin, T., Schulfter, K., Kiiskinen, H., Samela, J. \&Bismarck, A. (2012). High performance cellulose nanocomposites: comparing the reinforcing ability of bacterial cellulose and nanofibrillated cellulose. ACS applied materials \& interfaces 4(8): 4078-4086.

Li, Q. \&Renneckar, S. (2009). Molecularly thin nanoparticles from cellulose: isolation of sub-microfibrillar structures. Cellulose 16(6): 1025.

Li, Q. Q. (2012). Nanocellulose: Preparation, characterization, supramolecular modeling, and its life cycle assessment (Doctoral Dissertation, Virginia Tech).

Lin, N. \&Dufresne, A. (2014). Nanocellulose in biomedicine: Current status and future prospect. European Polymer Journal 59: 302-325.

Liu, D., Chen, X., Yue, Y., Chen, M. \&Wu, Q. (2011a). Structure and rheology of nanocrystalline cellulose. Carbohydrate Polymers 84(1): 316-322.

Missoum, K., Belgacem, M. N. \&Bras, J. (2013). Nanofibrillated cellulose surface modification: a review. Materials 6(5): 1745-1766. 
Moon, R. J., Martini, A., Nairn, J., Simonsen, J. \&Youngblood, J. (2011). Cellulose nanomaterials review: structure, properties and nanocomposites. Chemical Society Reviews 40(7): 3941-3994.

Nakagaito, A. \&Yano, H. (2004). The effect of morphological changes from pulp fiber towards nano-scale fibrillated cellulose on the mechanical properties of highstrength plant fiber based composites. Applied Physics A 78(4): 547-552.

Nakagaito, A. N. \&Yano, H. (2005). Novel high-strength biocomposites based on microfibrillated cellulose having nano-order-unit web-like network structure. Applied Physics A: Materials Science \& Processing 80(1): 155-159.

Pääkkö, M., Ankerfors, M., Kosonen, H., Nykänen, A., Ahola, S., Österberg, M., Ruokolainen, J., Laine, J., Larsson, P. T. \&Ikkala, O. (2007). Enzymatic hydrolysis combined with mechanical shearing and high-pressure homogenization for nanoscale cellulose fibrils and strong gels. Biomacromolecules 8(6): 19341941.

Pääkkö, M., Vapaavuori, J., Silvennoinen, R., Kosonen, H., Ankerfors, M., Lindström, T., Berglund, L. A. \&Ikkala, O. (2008). Long and entangled native cellulose I nanofibers allow flexible aerogels and hierarchically porous templates for functionalities. Soft Matter 4(12): 2492-2499.

Palza, H. (2015). Antimicrobial polymers with metal nanoparticles. International journal of molecular sciences 16(1): 2099-2116.

Postek, M. T., Vladár, A., Dagata, J., Farkas, N., Ming, B., Wagner, R., Raman, A., Moon, R. J., Sabo, R. \&Wegner, T. H. (2010). Development of the metrology and imaging of cellulose nanocrystals. Measurement Science and Technology 22(2): 024005.

Revol, J.-F., Bradford, H., Giasson, J., Marchessault, R. \&Gray, D. (1992). Helicoidal self-ordering of cellulose microfibrils in aqueous suspension. International journal of biological macromolecules 14(3): 170-172.

Rodríguez, K., Renneckar, S. \&Gatenholm, P. (2011). Biomimetic calcium phosphate crystal mineralization on electrospun cellulose-based scaffolds. ACS applied materials \& interfaces 3(3): 681-689.

Saito, T., Hirota, M., Tamura, N., Kimura, S., Fukuzumi, H., Heux, L. \&Isogai, A. (2009). Individualization of nano-sized plant cellulose fibrils by direct surface carboxylation using TEMPO catalyst under neutral conditions. Biomacromolecules 10(7): 1992-1996.

Saito, T. \&Isogai, A. (2006a). Introduction of aldehyde groups on surfaces of native cellulose fibers by TEMPO-mediated oxidation. Colloids and Surfaces A: Physicochemical and Engineering Aspects 289(1): 219-225.

Saito, T., Nishiyama, Y., Putaux, J.-L., Vignon, M. \&Isogai, A. (2006b). Homogeneous suspensions of individualized microfibrils from TEMPO-catalyzed oxidation of native cellulose. Biomacromolecules 7(6): 1687-1691.

Sakurada, I., Nukushina, Y. \&Ito, T. (1962). Experimental determination of the elastic modulus of crystalline regions in oriented polymers. Journal of Polymer Science 57(165): 651-660. 
Satyamurthy, P., Jain, P., Balasubramanya, R. H. \&Vigneshwaran, N. (2011). Preparation and characterization of cellulose nanowhiskers from cotton fibres by controlled microbial hydrolysis. Carbohydrate Polymers 83(1): 122-129.

Spence, K. L., Venditti, R. A., Rojas, O. J., Habibi, Y. \&Pawlak, J. J. (2011). A comparative study of energy consumption and physical properties of microfibrillated cellulose produced by different processing methods. Cellulose 18(4): 1097-1111.

Tingaut, P., Zimmermann, T. \&Sèbe, G. (2012). Cellulose nanocrystals and microfibrillated cellulose as building blocks for the design of hierarchical functional materials. Journal of Materials Chemistry 22(38): 20105-20111.

Turbak, A. F., Snyder, F.W., Sandberg, K.R. (1983). Microfibrillated cellulose, a new cellulose product: properties, uses, and commercial potential. J Appl Polym Sci 37: 815-827.

Wågberg, L., Winter, L., Ödberg, L. \&Lindström, T. (1987). On the charge stoichiometry upon adsorption of a cationic polyelectrolyte on cellulosic materials. Colloids and surfaces 27(1-3): 163-173.

Wei, H., Rodriguez, K., Renneckar, S. \&Vikesland, P. J. (2014). Environmental science and engineering applications of nanocellulose-based nanocomposites. Environmental Science: Nano 1(4): 302-316.

Wuhrmann, K., Heuberger, A. \&Muhlethaler, K. (1946). Electron-microscopic investigations of cellulose fibers after supersonic treatment. Experientia 2-2012: 105-107.

Yue, Y., Zhou, C., French, A. D., Xia, G., Han, G., Wang, Q. \&Wu, Q. (2012). Comparative properties of cellulose nano-crystals from native and mercerized cotton fibers. Cellulose 19(4): 1173-1187.

Zimmermann, T., Bordeanu, N. \&Strub, E. (2010). Properties of nanofibrillated cellulose from different raw materials and its reinforcement potential. Carbohydrate Polymers 79(4): 1086-1093. 


\section{CHAPTER 2: NANOCELLULOSE CURRENT POTENTIAL APPLICATIONS}

Increased concern about the environment and a consumer and market push for greater sustainability in the use of products and services are making the development and use of renewable materials and products one of the central priorities of this and coming decades. Resource constraints are driving a push toward bio-based products. Cellulose is the earth's most abundant organic substance, with an annual production in the biosphere of about 90 billion tons. Applications of nanotechnology to the manufacture of forest-based products promise new value added features, improved performance attributes, reduced energy intensity, and more efficient use of renewable and nonrenewable materials. Renewable and sustainable materials enabled by nanotechnology have the potential to reduce materials and energy consumption because of unnecessary over construction of products and structures,

arising from our current lack of knowledge of nano-scale structures and interfaces (Shatkin et al. 2014).

Because of their promising characteristics, nanocellulosic fibers have been widely utilized in a variety of applications in various fields, including medical, packaging, paper and coating, electronics and membranes.

De Moura et al. 2012 reported that cellulose based materials offer various advantages, such as edibility, biodegradability, barrier properties, non-toxicity and low cost. Industrial progression would exploit the ecological advantages of converting these valuable biomaterials into value added products for various applications, including the following examples:

\subsection{Nanocellulose-based nanocomposites}

When describing nanocellulose nanocomposite applications, it is focused on their potential environmental applications (e.g., antibacterial, catalytic, sensing, and energy). A range of different guest nanomaterials such as metal NPs ( $\mathrm{Au}, \mathrm{Ag}, \mathrm{Pd}, \mathrm{Ni}, \mathrm{TiO} 2, \mathrm{CuO}$ ), mineral nanomaterials (CaCO3, SiO2, montmorillonite), and carbonaceous nanomaterials (carbon nanotube, graphene) have been incorporated into or onto nanocellulose substrates (Wei et al. 2014). 
Cellulose based electro-active paper (EAPap) has been employed in many smart applications including electromechanical transducers, actuators, and for haptic applications (Kim et al. 2015). To improve the performance, cellulose based EAPap actuators have been characterized with ionic liquids, conducting polymer, SWNT/MWNT, chitosan blends, and metal oxide cellulose nanocomposites. Yang et al. fabricated a wirelessly driven CellulosePolypyrrole-Ionic Liquid (CPIL) nanocomposite actuator by incorporating nanoscale polypyrrole onto cellulose by an in situ polymerization technique followed by activation in a room temperature ionic liquid (Figure 2.1) (Yang et al. 2010)

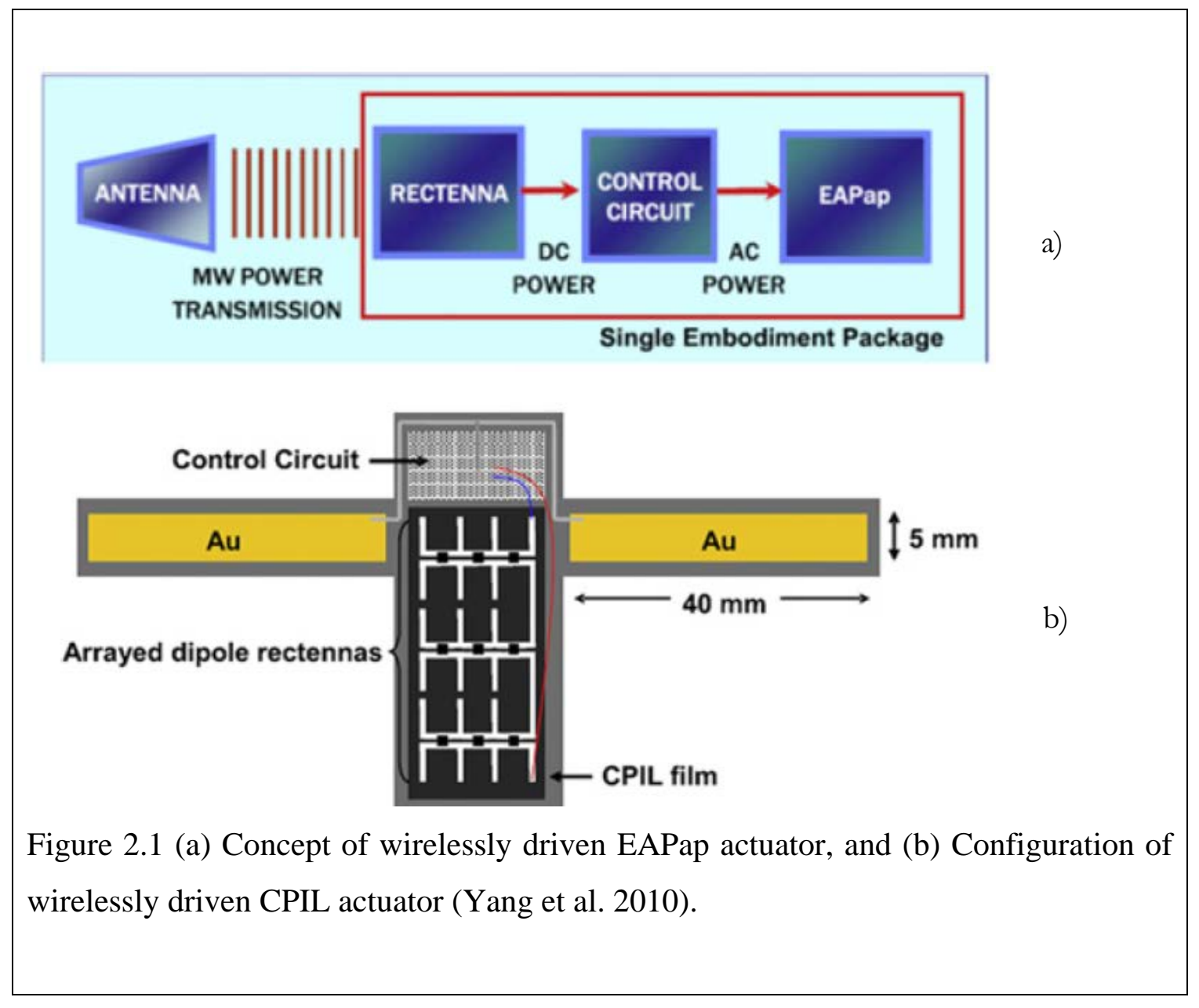

The material properties of cellulose for smart applications can be enhanced by adding metal oxide to the cellulose matrix. 
A high strength elastomeric nanocomposite was prepared by dispersing microcrystalline cellulose in a polyurethane matrix.The resulting large improvements in the stiffness/strength, as well as strain-to-failure, were supposed to be due to the good interaction, caused by both covalent and hydrogen bonds, between the polyurethane and the cellulose nanofibrils. A TiO2-cellulose nanocomposite was synthesized through titanyl sulfate hydrolysis in an acidic medium in the presence of cellulosic fibers.

By hybridizing functional metal oxides with cellulose substrates, nature friendly, cost effective and disposable sensor devices with good sensing performance are possible. A cellulose nanocomposite with high content of $\mathrm{Fe} 3 \mathrm{O} 4$ nanoparticles with regenerated cellulose as a matrix provided a green and facile method for the preparation of bio-based nanocomposite films with excellent magnetic properties.

A hybrid thin film consisting of tin oxide nanoparticles and cellulose was fabricated and characterized. The reduction in the crystalline melting transition temperature due to the $\mathrm{SnO} 2$ nanoparticles showed the potential application of this hybrid configuration as a biodegradable and flexible humidity sensor(Kim et al. 2015).

\subsection{Sensors (medical, environmental, and industrial)}

CNC films are hygroscopic or water-absorbing, but individual crystals do not dissolve or even swell in water. For this reason, is possible to use them in applications involving moisture (for example, humidity sensors) or for real-time contaminant detection. Cellulose nanomaterial-based sensors could help in monitoring structures like bridges to detect elevated stress. Modifying cellulose nanomaterials by double-walled carbon nanotubes and graphite carbon nano-powder expands applications for sensor applications (Wang et al. 2013). The electrical conductance of these materials displays a high sensitivity to strain when tensile stress is applied.

Electrospun PVA nanofibers mat reinforced with CNC have also been produced, which showed a fully reversible mechanical response under the effect of humidity cycles.Scanning electron microscopy (SEM) images revealed that the incorporation of CNs improved the 
morphological stability of the composite fibers even in high humidity environments (Peresin et al. 2010a).

Hybrid materials containing CNC were utilized as electrochemical sensors to selectively detect DNA hybridization. Silver nanoparticles were reported to be obtained onto TEMPOmediated oxidized $\mathrm{CNC}$ by using $\mathrm{NaBH} 4$ as a reducing agent. The presence of CNC proved to be useful in avoiding the aggregation of the nanoparticles (Liu et al. 2011b)

CNCs were used as morphology-inducer and coordinate agent for the synthesis of TiO2 nanocubes, in milder reaction conditions. Photocatalytic and photoelectronic properties of nanosized TiO2 particles, make them very attractive in application areas such as catalysis, gas sensors, solar energy conversions. TiO2 nano-cubes obtained with this method were uniform in size and with a narrow particle size distribution (Zhou et al. 2007).

Cellulose nanoplatelet gels were obtained through grafting amine-monomers on the surface of TEMPO-oxidized CNCby using double-click chemistry.Based on this approach, fluorescent coumarin and anthracene chromophores were attached to TEMPO-oxidized CNC via double-click chemistry. Potential applications of such materials include photo-recording devices, liquid crystal displays and additional photo-sensitive uses (Filpponen et al. 2011)

NC based smart materials display intelligent behavior in response to environmental stimuli such as light, temperature, electrical input, $\mathrm{pH}$, and magnetic force, to design smart materials for many applications. Additionally, due to biocompatible and biodegradable nature, cellulose based stimuli-responsive materials have shown great potential in electro-stimulated targeted drug delivery systems (Zhang et al. 2011).

NFC photo-responsive nature can act as an adaptive filler in a soft polymer matrix, and can also act as a photo-switchable gelator (Kettunen et al. 2011).

The exceptional characteristics of NC such as low density, thermal stability, chemical resistance, high mechanical strength, biocompatibility and bio degradability has allowed to use it as functional material in actuation systems, and sensors for detecting $\mathrm{pH}$, organic vapors, ions and humidity.(Kim et al. 2015) 
NC smart composites can form stimuli responsive materials for sensory and actuation applications, devices for electro-stimulated drug release, and biocompatible energy harvesting. CNCs have been widely used as reinforcement in shape memory materials, which changes their shape upon external stimulus.(Auad et al. 2008)

The rigidity of shape memory polymers by adding small amounts of well-dispersed NC was improved significantly. A stimuli-responsive mechanically adaptive CNCs nanocomposite was inspired by biological sea cucumbers, which has the potential to reversibly change the stiffness of their dermits.(Capadona et al. 2008)

The shape memory properties of smart polyurethane were modified by reinforcing Polyaniline (PANi)-coated cellulose nano fibers.(Auad et al. 2011)

The two-phase structure of the polymer is responsible for the material's ability to remember and autonomously recover its original shape after being deformed in response to an external thermal stimulus. The use of conductive CNFs opens the future possibility of triggering the shape memory response of these cellulose-PU composites through the use of a stimulus other than temperature, although PANi deposition should be optimized for that purpose.

Combined with the ability to accommodate other functional materials, cellulose has many opportunities for applications in electrical, electrochemical, and optical devices. Using nanoscaled cellulose fibers, the advanced application has been suggested and studied. During past decade, research on nanostructures of cellulose has increased dramatically due to the potential applications in electronics, biosensors, and energy storage devices. Development of nanostructured inorganic materials in the form of nanocrystals, nanowires, and nanotubes provides a list of functional inks for integration into paper (Tobjörk and Österbacka 2011) 


\subsection{Electronics}

Cellulose nanofibrils (CNF) are being used in the development of next generation flexible TV screen, computer screen, personal data assistant (PDA) screen, and other advertising display systems (Thavasi et al. 2008)

Considering its high transparency and flexibility, as well as desirable electrical properties, $\mathrm{CNF}$ is an ideal candidate as an alternative and ecofriendly substrate for electronics (Gao et al. 2013; Huang et al. 2013; Nagashima et al. 2014; Zhou et al. 2014).

Optically transparent nano-composite films of bacterial cellulose and/or CNF in combination with resins have been fabricated and used as a substrate for the OLED displays (Ummartyotin et al. 2012, Okahisa et al. 2009). The nanocomposite had appealing features of flexibility, optical transparency, and high light transmittance of up to $80 \%$.

Successful depositions of transparent and conductive materials, including tin-doped indium oxide, carbon nanotubes and silver nano-wires, have been achieved on nano-paper substrates, opening up a wide range of applications in optoelectronics, such as displays, touch screens, and interactive paper (Hu et al. 2013). The biodegradability of nanocellulose might aid in the recyclability of these devices to recover the valuable metallic components.

Cellulose is very interesting for an energy storage system due to its structural advantage. Using liquid electrolytes, ionic species can move between the electrode surfaces due to porosity in cellulose at room temperature condition. As an electrode on cellulose, conducting materials such as conductive metal oxide, graphene, carbon nanotubes (CNTs), metal nanowires, and conducting polymers have been suggested and investigated (Zheng et al. 2013).

As a promising candidate for energy application using $\mathrm{NC}$, the cellulose based flexible energy storage device was made by using cellulose and MWCNT (Pushparaj et al. 2007). 
The simple structure is based on a single sheet of conductive cellulose paper (separator) made from room temperature ionic liquid and CNT (electrode). The as-fabricated flexible lithiumion battery exhibited a specific capacity of $110 \mathrm{mAh} / \mathrm{g}$ by using aqueous $6 \mathrm{M} \mathrm{KOH}$. Figure 2.2 shows the schematic of cellulose based flexible energy storage.

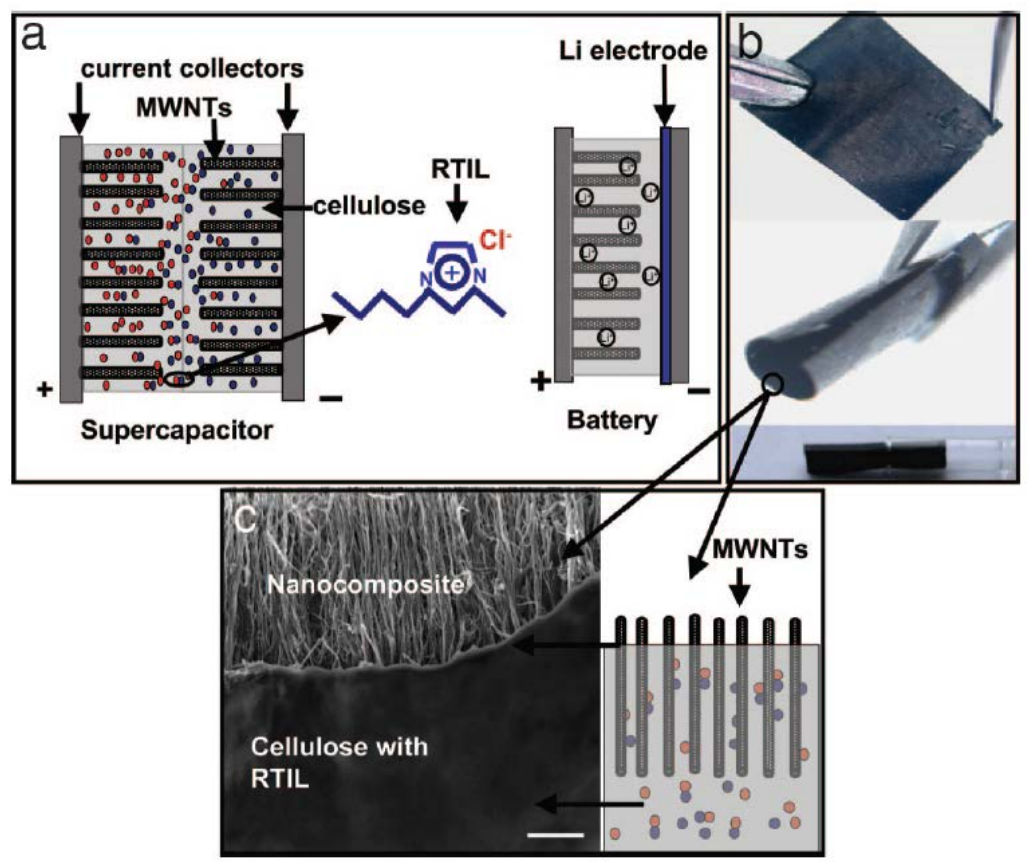

Figure 2.2 Cellulose based supercapacitor and battery (Pushparaj et al. 2007).

Cellulose in available nanofibers, nanocrystals and its derivatives also has been suggested for high power Li-ion battery (LIB) application as separators, electrolytes and electrodes (Jabbour et al. 2013).

Using microfibrillated cellulose as reinforcement for LIB polymer electrolytes, composite membranes with excellent mechanical properties ( $~ \sim 80 \mathrm{MPa}$ ), electrical high ionic conductivity (approaching $103 \mathrm{~S} / \mathrm{cm}$ ), and stable overall electrochemical performances was also reported (Chiappone et al. 2011). 
Very recently, a nanofibrillated cellulose composite with a liquid electrolyte was reported for a very high elastic modulus around $400 \mathrm{MPa}$ while the values of ionic conductivity is about 5×10-5 S/cm for LIB application (Willgert et al. 2014).

Zhu et al. 2013 investigated sodium-ion battery (SIB) using natural wood fiber as electrolyte reservoir. The mesoporous structure of wood fiber as an electrolyte reservoir that allows for ion transport through the outer and inner surface of the fiber. A stable cycling performance with an initial capacity of $339 \mathrm{mAh} / \mathrm{g}$ was successfully demonstrated. The new approach will be utilized for low cost Na-ion based batteries.

Hess et al. reported microelectromechanical systems (MEMS) based on poly(vinyl acetate)CNC reinforced materials towards the development of intracortical neural recording probes (Hess et al. 2011). Chiral nematic suspensions of cellulose were prepared from dissolving grade wood pulp by Edgar et al. On evaporation of the water, the suspension dries down to give iridescent cellulose optically active films. The optical properties of the films can be modified by structural changes to the films and preparation conditions (Edgar and Gray 2001).

CNC has been used as stabilizer due to their smaller size, and inherent higher specific area compared to microcrystalline cellulose which had been utilized for many years as stabilizer of oil in water $(\mathrm{o} / \mathrm{w})$ emulsions for the food industry and also as foam stabilizer. Kalashnikova et al. 2011 used unmodified nanocrystalline cellulose to stabilize hexane/water interface. The emulsions were reported stable for several months, in the form of a monodispersed emulsion with defined (4 $\mu \mathrm{m}$ in diameter) oil in water $(\mathrm{o} / \mathrm{w})$ droplets providing a promising, healthier and environmentally friendlier alternative to synthetic surfactants.

CNC were reported to be used as reinforcing agent of hemicelluloses hydrogels. CNC were first coated with 2-hydroxyethylmethacrylate modified hemicelluloses and methacrylic functional groups were grafted from the surface, serving as a template for polymerization and nextwork formation.Hydrogels with excellent mechanical, recovery, water holding 
capacity were superior to hydrogels obtained by traditional methods and with no CNC addition. Furthermore, some of the measured properties were comparable to natural tissue with hydrogel-like properties, thus this material is envisioned as a potential replacement for articular cartilage (Karaaslan et al. 2011).

Among the different polymeric matrices reinforced by different approaches with CNC, poly(vinyl alcohol) is one of the polymeric matrices more used to be reinforced with CNC, in both cast films and electrospun nonwovensdue to its natural affinity through hydrogen bonding (Peresin et al. 2010).

Barrier Property has been increased by adding CNC or NFC. One factor for controlling barrier properties is increasing crystallinity of the biopolymers.The barrier properties of paper could be improved by adding MFC extracted from wood. More recently, adding NFCs on biodegradable Poly(ethylene oxide) or (PEO) s increased gas barrier properties. By simple solvent casting, crystalline orientation of PEO was improved as well as permeation wall to gas were effectively formed by 5\% NFC addition (Fukuya et al. 2014).

Cellulose nanomaterials are anticipated to be useful as a barrier in grease-proof types of papers and as a wet-end additive to enhance retention of additives, as well as dry and wet strength, in commodity types of paper and board products. Further, the addition of CNF to coatings improves ink adhesion to the surface, allowing papers to be thinner and lighter (Aulin et al. 2010).

\subsection{Antibacterial/Antimicrobial}

In some of the latest research, nanocellulose filters have shown the capacity to remove virus from water at levels that match the best industrial virus filters. One big challenge for the application of filter membranes in water treatment is surface biofouling. Due to their high surface to volume ratio, nanocelluloses possess the capacity to house greater numbers of AgNPs in a more homogeneous state. The addition of antimicrobial materials such as AgNPs to the filter membrane can inhibit biofilm growth and prolong filter life. AgNP decorated MFC films, hydrogels, and aerogels can be readily prepared by UV reduction of Ag+ in the corresponding matrix. Xiao et al. used UV irradiation for the fabrication of AgNP/nanocellulose nano-composites (Xiao et al. 2013) 
This AgNP/MFC nanocomposite can inhibit the growth of Escherichia coli and Staphylococcus aureus whereas the MFC didn't show any inhibition effects (Dong et al. 2013a).

In addition to UV irradiation, the application of reducing agents has been uses an alternative way to reduce Ag+ to AgNPs. Barud et al. incorporated AgNPs on BC membranes by reduction of AgNO3 in the presence of triethanolamine (TEA). In that work TEA acted as both reducing and chelating agent, influencing Ag nucleation and growth. The AgNP/BC nanocomposites displayed strong antimicrobial activity towards S. aureus, P. aeruginosa, and E. coli.(Barud et al. 2011).

Ag+ has also been reduced by the hydroxyl groups on BC at elevated temperature (353 K).The AgNP/BC nanocomposites produced by this technique inhibited the growth of $S$. aureus for $72 \mathrm{~h}$ and thus have potential for many antimicrobial applications (Yang et al. 2012).

Because of the enormous potential of cellulose materials, it is of great interest to develop novel functional cellulose-based biomaterials by introducing antibacterial/antimicrobial activities through the functionalization and/or incorporation of antibacterial/antimicrobial agents. For instance, antimicrobial nanocellulose-based materials have been obtained by the incorporation of N-halamine (Sun et al. 2010) and nanocurcumin (Raghavendra et al. 2014) into the cellulose network and by the chemical grafting of aminoalkyl groups (Fernandes et al. 2013), 2-benzyl-4- chlorophenol (Cassano et al. 2013), L-cysteine (Caldeira et al. 2013), and diclofenac (Cassano et al. 2010) onto the surface of the cellulose backbone.

In one study, an electrospinning technique was successfully used to prepare composite nanofiber fabrics with CA nanofibers and the N-halamine antimicrobial agent of bis(Nchloro-2,2,6,6-tetramethyl- 4-piperidinyl) sebacate (Cl-BTMP) (Sun et al. 2010). The electrospun composite nanofiber fabrics containing Cl-BTMP showed significant antimicrobial activity against Staphylococcus aureus (Gram positive), Escherichia coli (Gram negative), and Pseudomonas aeruginosa (Gram negative) compared to the control 
solution cast films; this was clearly attributed to the aggregation of Cl-BTMP in the solutioncast samples.

More recently, Raghavendra et al. impregnated nanocurcumin (curcumin NPs) into cotton nanocellulose fibers for potential antimicrobial applications. The cumulative release studies indicated that the fibers released all of the nanocurcumin at about $60 \mathrm{~h}$. Moreover, the antimicrobial activity results show an effective antimicrobial against E. coli and S.aureus over a period of $24 \mathrm{~h}$ (Raghavendra et al. 2014).

In 2013, Fernandes et al. developed bio-inspired antimicrobial nanocellulose membranes by the chemical grafting of aminoalkyl groups on the surface of bacterial cellulose for biomedical applications. The aminoalkyl-grafted bacterial nanocellulose (BNC-NH2) membranes were prepared in three steps: (1) hydrolysis of the silane derivative, (2) adsorption of the hydrolyzed species onto BNC nanofibrils, and (3) a chemical condensation reaction. The BNC-NH2 membranes showed a significant reduction in bacterial cells for both E. coli and S.aureus after 24 h, whereas the BNC membranes without functionalization showed no reduction in bacterial viability (Fernandes et al. 2013).

Similarly, Caldeira et al. reported nanocellulosic fibers with effective antibacterial properties obtained by the surface functionalization of 2,2,6,6-tetramethylpiperidine-1-oxyl radical activated cotton cellulose fibers with L-cysteine (Caldeira et al. 2013).

Butchosa et al. reported cellulosebased NCs with antibacterial activity, simply by combining BNC nanofibers and chitin nanocrystals (Butchosa et al. 2013).

In the last 5 years, several approaches have been successfully developed to incorporate metal particles into nanocellulose materials for antibacterial activity, such as the physical blending of NPs with cellulose and in situ sol-gel formation of metal particles within cellulose materials.For example, silver particles have been used as potential agents with a broad antibacterial activity and low presumed toxicity to coat cellulosic materials for biomedical applications. Li et al. reported the microwave-assisted synthesis of silver/cellulose NCs with high antibacterial properties against E. coli (Gram-negative bacteria) and S. aureus (Gram- 
positive bacteria). The silver/cellulose NCs prepared by the formation of Ag NP through the in situ reduction of silver nitrate in ethylene glycol on the surface of microcrystalline cellulose (MCC) (Li et al. 2011).

Although metal NPs have been associated with remarkable antibacterial properties, safer and greener approaches using the reduction of metal slats are still limited in the literature. Recently, researchers have reported different preparations of metal-coated cellulose materials without using toxic chemical reductants such as $\mathrm{NaBH} 4$ as a reducing agent for the metal salts. For instance, Barua et al. recently reported the preparation of copper-copper oxide $(\mathrm{Cu}-\mathrm{CuO}) \mathrm{NP}$-coated $\mathrm{CNFs}$ through a green reductive technique by ethanolic extracts of the Terminalia chebula fruit. The $\mathrm{Cu}-\mathrm{CuO}$ NP-coated cellulose materials exhibited promising antimicrobial activity against Gram-positive and Gram-negative bacteria and fungal species (Barua et al. 2013).

Salmieri et al. demonstrated the strong antimicrobial potential of films prepared by using poly (lactic acid) (PLA) containing cellulose nanocrystals (CNC) coated with nisin as a promising bioactive packaging for protecting fresh food products against foodborne pathogens. The film was prepared using the compression moulding method (Salmieri et al. 2014).

\subsection{Biomedical Applications}

Several research groups have developed BNC-based implants, which conform to blood and tissue compatibility, endothelization, cell ingrowth, surgical handling, and common methods of sterilization (Gatenholm and Klemm 2010). Klemm et al.in a series of investigations have developed prototypes of BNC tubes (brand name BASYC, Bacterial Synthesized Cellulose) with different diameters which can be used for arterial grafting applications. The wall of the BASYC tubes consist of BNC loaded with $90 \%$ water or more. The initial studies showed that the BNC tubes have very good surgical handling and can be sterilized in standard ways. In a follow-up in vivo study with rats, pigs, and sheep, the BNC tubes were successfully used to replace carotid arteries (Klemm et al. 2001; Wippermann et al. 2009). 
The first animal study of the carotid artery-BASYC complex on rats showed good biocompatibility and incorporation into the body without any rejection after 4 weeks (Klemm et al. 2001; Schumann et al. 2009).

Nanocellulosic materials have also been used in other biomedical applications, such as cancer targeting (Dong et al. 2014),cornea replacement (Wang et al. 2010),biological detection (Qin et al. 2014),and biology-device interfaces (Shi et al. 2014).In one example, CNCs were used for the targeted delivery of chemotherapeutic agents to cancer cells.Briefly, Dong et al. synthesized folic acid (FA) grafted CNCs for the first time and explored in vitro their folate-receptor-mediated uptake by human and rat brain tumor cells. The reaction pathway scheme used for the synthesis of FA-grafted CNCs is shown in Figure 2.3 (Dong et al. 2014)

In another example, $\mathrm{Wu}$ et al. reported nanogel complexes made of poly(N-isopropyl acrylamide-co-butyl methacrylate) (PNB) and different contents of BNC with a surfactantfree emulsion polymerization. These nanogel biomaterials showed reversible thermosensitive phase behaviors from a swollen gel to shrunken gel with increasing temperature.These nanogel complexes may be an ideal candidate for a wide range of medical applications, such as injectable biomaterials and vascular embolization interventional therapies (Wu et al. 2013).

Recently, Shi et al. 2014 reported electroactive hydrogels in which conductive polymers and BNC were combined to build a biology-device interface. In this study, polyaniline or polypyrrole were electrochemically polymerized onto the BNC hydrogel surface; this resulted in a biphasic Janus hydrogel with voltage responsive properties. Such hydrogels that respond to voltage changes can provide an interface for integrating microelectronics with biology to build implantable devices for future regenerative medicine. More recently, BNC has also been used as a living membrane system for recombinant bacterial strains (e.g., $E$. coli) for potential applications in biological and chemical detection (Qin et al. 2014). 


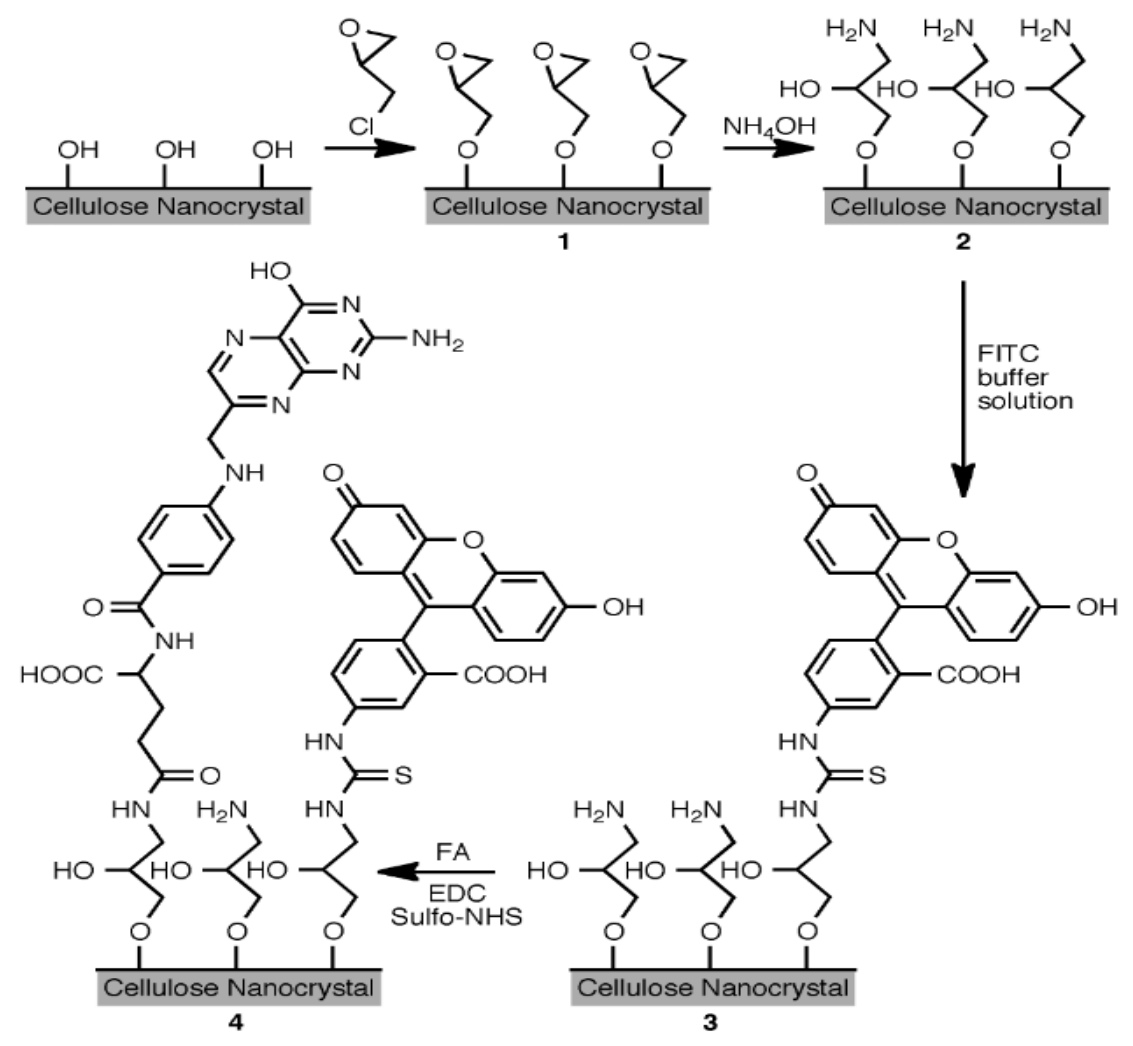

Figure 2.3 Synthesis of FITC-Labeled, Folic Acid-Conjugated CNCs (Dong et al. 2014).

Chang and Wang recently developed hydrogen peroxide (H2O2) and oxygen (O2) releasing microfibrillated cellulose (MFC) based NCs that modulate the growth of mammalian cells. In this investigation, calcium peroxide (CPO) was embedded into highly porous MFC NCs to produce $\mathrm{H} 2 \mathrm{O} 2$, whereas catalase was added to convert the generated $\mathrm{H} 2 \mathrm{O} 2$ to $\mathrm{O} 2$ under physiological conditions [Figure 2.4].In vitro cell culture studies for 1 and 5 days showed that cell attachment was decreased and cell proliferation was delayed in MFC/CPO NCs, clearly because of the toxicity of $\mathrm{H} 2 \mathrm{O} 2$ and/or hydroxyl radicals. On the other hand, cell survival was significantly increased in the case of NCs in contact with CPO and catalase; this indicated the effective conversion of $\mathrm{H} 2 \mathrm{O} 2$ to $\mathrm{O} 2$, which provided nutrition for cell growth and proliferation for up to 5 days (Chang and Wang 2013). 

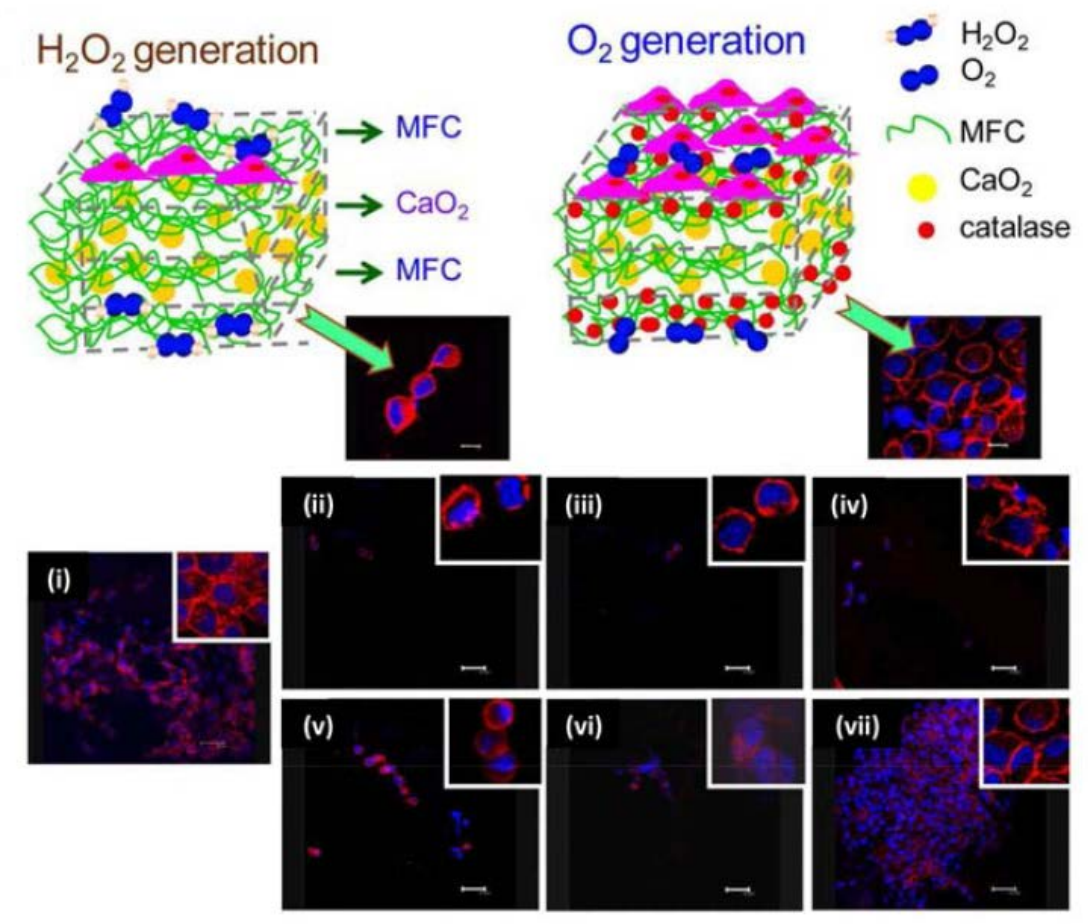

Figure 2.4 Cell morphology of the (i) pristine MFC. MFC/CPO-X with different CPO concentrations: (ii) 5, (iii) 10, and (iv) 15 wt \%. MFC/[CPO1catalase] with different CPO concentrations: (v) 5, (vi) 10, and (vii) 15\% w/w. Scale bar540 lm. Reproduced with permission from (Chang and Wang 2013).

\subsection{Implants}

Nanocellulose can also be used in medical implants. In recent years several metal-based materials have been used for medical implantation applications. The commonest examples of materials being used for medical implantation are silicon, titanium, platinum, gold, iridium oxide, glassy carbon, and stainless steel which still have the widespread use e.g. as cortical implants (Bellamkonda et al. 2012).

It has been supposed that the mechanical mismatch between the implanted electrode and the brain tissue plays a significant role to in the cell-mediated inflammatory response. As mentioned before, most neural interfaces are made from metals that are much stiffer than brain tissue (Capadona et al. 2012). Such stiff probes are easily inserted into the soft 
brain tissue without buckling because of their high stiffness, but micromotion between the probes and brain tissue may significantly increase the risk of tissue damage and trigger an immune response that can result in the formation of an insulting cellular sheath (gliosis), a chronic reactive biological response to the foreign probe; this leads to death of neurons and the encapsulation of the probe (Levesque et al. 2010).

Researchers developed a new class of biologically inspired, mechanically adaptive cellulose nanocomposites (NCs) that can controllably and selectively be switched between stiff and soft states.The design of these materials was inspired by the architecture of the sea cucumber dermis (Capadona et al. 2007; Shanmuganathan et al. 2010). These invertebrates have the fascinating ability to rapidly and reversibly switch the stiffness of their skin (from 5 MPa under normal conditions to 50 MPa under threat; Figure 2.5) (Trotter et al. 2000). This design is achieved through an NC that relies on stiff collagen fibers dispersed throughout a soft fibrillin matrix (Takehana et al. 2014). The sea cucumber dermis behavior offers a rich source of inspiration for the development of a series of polymer NCs with switchable stiffness.

Capadona and colleagues developed the first generation of mechanically adaptive cellulose-based NCs. In this system, CNCs isolated from tunicate sea creatures were integrated into a rubbery ethylene oxide-epichlorohydrin copolymer matrix (Capadona et al. 2008; Capadona et al. 2007) . However, the stiffness of the most rigid ethylene oxideepichlorohydrin/CNC NC was lower ( $800 \mathrm{MPa}$ ) than desirable for the targeted fabrication of electrodes that could be inserted into the brain through the piamater, the membrane surrounding the brain.

Therefore, the second generation of mechanically adaptive materials was designed on the basis of an amorphous polymer, poly(vinyl acetate) (PVAc), and CNCs isolated from tunicates as reinforcing fillers (Shanmuganathan et al. 2009; Shanmuganathan et al. 2010). 
Standard state

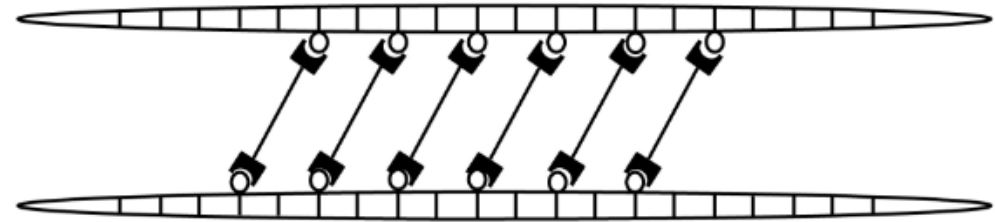

Soft state
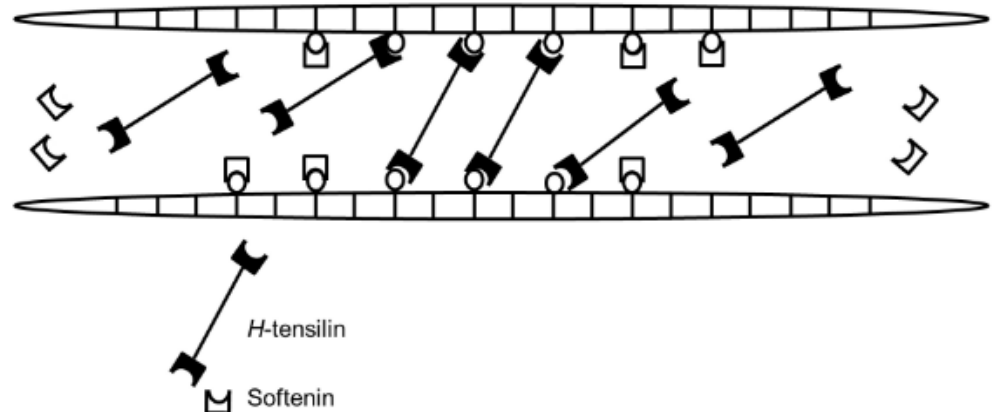

Binding site for $\mathrm{H}$-tensilin and softenin

Collagen fibril

Figure 2.5 Hypothetical model of the stiffness change mechanism in the sea cucumber dermis (Takehana et al. 2014).

Harris et al. reported the first in vivo application of these mechanically switchable materials as substrates for penetrating brain implants; they have shown that the dynamic polymer NC is sufficiently stiff to penetrate the cortical tissue without the need for assistive devices.NC implants consisted of poly(vinyl acetate) and cellulose nanocrystals derived from tunicates (tunicate whiskers or whiskers) that were created by casting films from a dimethylformamide (DMF) solution of the polymer and whiskers. Their study shows that the stiffness of the NC facilitates insertion into the cortex of a living rodent, the NC mechanically morphs within the animal to achieve a compliant state more similar to the mechanical properties of the brain, and the NC can integrate within the cortical tissue over many weeks (Harris et al. 2011).

Ex vivostudies confirmed that the stiffness of initially stiff NCs rapidly decreased when they were implanted into the rodent brain to more closely match the brain tissue 
Jorfi et al. reported mechanically adaptive bionanocomposites based on poly(vinyl alcohol) (PVOH) and cellulose nanocrystals (CNCs) derived from tunicates or cotton as the filler (Jorfi et al. 2013). Jorfi et al. recently developed physiologically responsive, mechanically adaptive polymer implants based on poly(vinyl alcohol) (PVA) and CNC derived from tunicates. The implant had the capability to also locally release the antioxidant curcumin. Their study showed that cellulose-based bionanocomposites could be combined with bioactive molecules to build multicomponent, biologically relevant biomedical implants for specific medical applications (Potter et al. 2014).

In recent years, there has been an increasing amount of interest in the development of nanocellulose-based biomaterials for soft-tissue replacement and reconstruction. The discovery of suitable biomedical materials for soft-tissue replacement and reconstruction applications is an important aspect for the development of medical implants. These medical implants should not only have similar mechanical characteristics as the tissue it replaces but also show improved biocompatibility, nonthrombogenic, sterilizability, durability, life span, lesser degrees of calcification, and good processability for ease of manufacturing (Puskas and Chen 2004). The implant should be biocompatible with the host tissues in terms of chemical, mechanical, surface chemistry, and pharmacological properties.Nondegradable biomaterials based on BC provide durable mechanical properties and long-term chemical stability in contrast to their counterparts, degradable ones.

High tensile mechanical properties, hydrogel-like behavior as cellulose interacts with surrounding media, such as water, nondegradable under physiological conditions and biocompatible key characteristics make BNC an exciting biomaterial candidate for pharmaceutical and biomedical applications, including blood vessel and articular cartilage tissue engineering (Bodin et al. 2007; Czaja et al. 2007; Malm et al. 2012; Petersen and Gatenholm 2011; Svensson et al. 2005). the small pore size of BC provides enough porosity of the material allowing cells to penetrate and migrate throughout the throughout the scaffold , makes BC a good candidate for scaffold applications (Bäckdahl et al. 2008; Bäckdahl et al. 2006; Klemm et al. 2005; Rambo et al. 2008). 
Nimeskern et al. 2013 demonstrated a biofabrication process to produce patient-specific BNC auricular implants; the ear-shaped BNC prototype material, was produced from a negative ear mold with a MRI scanning technique. Before the fabrication, the mechanical properties of native ear cartilage were measured in order to set a preliminary benchmark for ear cartilage replacement materials (Nimeskern et al. 2013). Based on their work, BNC has the capability to reach mechanical properties of relevance for ear cartilage replacement, and can be produced in patient-specific ear shapes.

\subsection{Tissue Engineering}

Disease, injury, or trauma can damage tissues, so treatments are needed to facilitate the repair, replacement, or regeneration of damaged tissues in the human body. In this context, typical treatments include tissue transplantation from one site of the body to another or from one individual to another. However, major problems associated with these techniques includes painful operation, high cost, risk of refusal by the patient's immune system, and risk of introducing infection or disease from the donor to the patient. Tissue engineering aims to regenerate damaged tissues without replacing them by combining body cells on a highly porous scaffold biomaterial, which acts as a template for tissue regeneration. These templates can provide biodegradability, biocompatibility, and other mechanical features. Moreover, the high porosity and interconnected pore structure can facilitate the satisfactory diffusion of nutrients to cells within the template and to the extracellular medium formed by these cells (O'brien 2011).

Due to to the unique three-dimensional (3D) network formed by cellulose and its mechanical properties and potential biocompatibility, cellulose is an ideal material candidate for a variety of tissue engineering applications (Helenius et al. 2006; Svensson et al. 2005; Watanabe et al. 1993).

Although, diverse cellulose species have been used to fabricate bionanocomposites containing hydroxy-apatite (HA), it seems that BNC is the most promising material for potential tissue engineering, mostly because of its low cytotoxicity and high porosity. Therefore, the majority of recent studies for tissue engineering applications have used BNC, 
a cellulosic material with unique properties among other biomaterials that is used in tissue engineering scaffolds. Watanabe et al. investigated the biocompatibility of BNC in cell cultures. In this study, a new mammalian cell culture substrate was developed with anAcetobacter acetiproduced BNC. In this research, the authors showed that a serum-soaked BNC mem-brane was an effective substrate for use in tissue engineering (Watanabe et al. 1993).

Berti et al. 2013 reported that immortalized human vein endothelial cells presented a different behavior when they were cultured on two distinctly different BNC surfaces. The results show that although both BNC surfaces maintained viable endothelial cells, the porous BNC surface sustained more viable cells compared to the entangled surface at 20 days of culturing; this suggested that the fiber network arrangement or density was the responsible factor for endothelial cell differential behavior. Highly porous cellulose scaffolds based on pectin, CMC, and MFC were also fabricated by a lyophilization method. In vitro studies exhibited highest cell viability for these porous composite scaffolds (Ninan et al. 2013).

Polymer-based scaffolds for bone tissue engineering often fail to maintain high mechanical strength stability and mostly require chemical crosslinking to stabilize such scaffolds. Therefore, it is often a challenge to create load-bearing scaffolds for bone tissue engineering applications. The key attractive advantages of cellulose for bone tissue engineering are its mechanical properties (to tolerate local forces) and biocompatibility (to integrate with host tissue without immune responses).

Recently, Kumbar and coworkers, in a series of contributions, developed mechanically competent cellulose scaffold materials for bone tissue engineering applications (Aravamudhan et al. 2013; Kumbar et al. 2011). In this study, CA and ethyl cellulose microspheres were fabricated via an oil-in-water emulsion-sol-vent evaporation technique and were then sintered together into 3D porous scaffolds with a solvent-nonsolvent sintering approach. These scaffolds showed mechanical properties in the midrange of human trabecular bone and were superior to several current polymer-based bone tissue engineering 
scaffolds with similar pore properties under both dry and physiological (wet) conditions. These nanocellulose-based scaffolds functionalized with collagen exhibited better human osteoblast adhesion, proliferation, and alkaline phosphatase expression compared to control PLGA-based scaffolds and, thereby, may serve as potential alternatives to other polymerbased scaffolds for bone tissue engineering (Aravamudhan et al. 2013).

HA is a well-known bone replacement biomaterial because of its properties, including its biocompatibility with the human body, bioactivity, osteoconductivity, and noninflammatory properties. HA/cellulose NCs have been extensively investigated in recent years for potential tissue engineering applications (Grande et al. 2009; Jia et al. 2012; Mucalo et al. 2009; Rodríguez et al. 2011; Shi et al. 2009; Zimmermann et al. 2011).

For example, HA/BNC NC scaffolds were prepared by a biomimetic approach for bonehealing applications (Zimmermann et al. 2011). In this study, CMC was adsorbed onto the BNC surface to induce nucleation of calcium-deficient HA and then treated with simulated body fluid (SBF) over a one-week period.In vitro experiments indicated that the presence of calcium-deficient HA crystals on the BNC surfaces increased cell attachment and the alkaline phosphatase (ALP) activity of bone cells. In a similar approach, Rodriguez et al. reported electrospun regenerated cellulose (RC)-based scaffolds with the ability to nucleate bioactive calcium phosphate crystals for potential bone-healing applications (Rodríguez et al. 2011).

Saska et al. developed compo-sites based on BNC and type I collagen (COL) for potential bone tissue engineering, in which collagen was homogeneously and covalently introduced into the BNC network to improve the biological properties of BNC for potential medical applications, including tissue engineering and regeneration (Saska et al. 2012).

High-aspect-ratio CNCs derived from the tunicate Halocynthia roretzi were deposited on to glass substrates by a simple spin-coating technique. It was found that myoblasts (muscle cells) were able to effectively sense the CNC surface topography and orientate relative to the bulk direction of the CNC orientation (Dugan et al. 2010). 
TEMPO-oxidized CNC were grafted with single stranded oligonucleotides with an amino modifier. After this and using the molecular recognition ability of the oligomeric base pairs, they were duplexed with complementary oligonucleotides grafted onto separate CNC batch. With this study, the concept of self-assembly of CNC with alternative bio-nanoparticles via DNA-coupling is proven. Such hybrid materials have a wide potential in the areas of tissue engineering and bio-medicine (Mangalam 2008).

\subsection{Wound Healing}

Bacterial nanocellulose (BNC) has been shown to be a superior candidate for conventional wound-dressing materials. BNC-based dressing materials, such as XCell, Bioprocess, and Biofill, are already commercially on the market for topical application in wound healing (Petersen and Gatenholm 2011).

The applications of BNC-based materials for skin-tissue repair have been reviewed already elsewhere (Fu et al. 2013a). The results of many studies indicate that topical applications of BNC improve the healing process of burns and chronic wounds. Czaja et al. used BNC membranes to treat patients with severe second degree burns. This study showed that the skin of the patients whose burns were covered with a BNC membrane healed faster than the wounds of patients who received conventional wound dressings. Czaja et al. discovered that never-dried BNCs showed remarkable conformability to various body counters, maintained a proper water balance, and significantly reduced wound pain (Czaja et al. 2007). More recently, animal studies by Fuet al. also confirmed the faster tissue regeneration, better healing effect, and lower inflammatory response of BNC-based dressing materials(Fu et al. 2013b).

In a recent study by Fu et al. 2012, BNC has been used as a potential skin-tissue repair material in vivo to replace conventional gauze dressings. Pathological studies showed better and faster healing effects and less inflammatory response in the thick-BNC group after 14 days compared to the other groups (i.e., the control and thin-BNC groups). Histological studies exhibited significant tissue regeneration, capillary formation, and cell proliferation in 
the wound area in the thick-BNC group on day 7 compared to the other groups.On the basis of this study, the wound healing was faster in the thick-BNC group than in the thin-BNC groups. This indicated the influence of the BNC thickness when it acted as a wound-dressing material (Fu et al. 2012).

CMC also has potential for use in wound-dressing materials. Fan et al. showed that a in situ crosslinked hydrogel made through the crosslinking of oxidized carboxymethylcellulose (OCMC) and carboxymethyl chitosan were capable of healing second-degree burns without any significant adverse reactions at 14 days post wounding with a rat model (Fan et al. 2013).

\subsection{Pharmaceuticals- Excipients}

Nanocellulose drug carriers may be produced in a bid to fight various types of illness-causing bacteria such as the ones that are resistant to antibiotics. The biocompatibility and biodegradability of nanocellulose is behind innovations to incorporate it into drugs and pharmaceutical delivery (Kolakovic et al. 2011). Powdered cellulose nanomaterials have also been suggested as an excipient in pharmaceutical compositions.

\subsection{Personal care - Cosmetics}

Cellulose nanomaterials may be used as a hydrating agent and non-allergenic rheology modifier, as well as for composite coating agents, in cosmetics (e.g., for hair, eyelashes, eyebrows, or nails). Demand for natural products, as well as high-performance colorants, increase the demand for these products.

\subsection{Nanocellulose paper and film}

The method for preparation of nanocellulose paper is built upon the common methods for paper production employed by the paper industry. Generally speaking, a nanocellulose suspension is filtered through a porous substrate and then the cellulose mat obtained on the substrate is pressed and dried to make paper. The only differences between nanocellulose 
paper and common cellulose paper are the diameter of the fibers used and the interstices (e.g., porosity) of the resultant paper. Because the nanocellulose diameter and the interstices in the nanocellulose paper are much smaller than the wavelength of visible light, less scattering occurs when light illuminates nanocellulose paper. For this reason, transparent nanocellulose papers can be readily produced (Wei et al. 2014) .

Sehaqui et al. 2010 made nanocellulose papers by filtration of $0.2 \%$ MFC suspension through a $0.65 \mu \mathrm{m}$ pore size filter membrane. The wet gel cake was clamped between woven metal cloth and carrier board and then dried at $363 \mathrm{~K}$ and $70 \mathrm{mbar}$ in a Rapid-Kothen semiautomatic sheet former. The papers obtained were flat and transparent and show greater mechanical strength than those pre-pared by suspension casting methods. Hu et al. carried out the first study on the optical properties of nanopaper substrates by a similar approach to make a transparent nanocellulose paper(Hu et al. 2013).

Henriksson et al. used different molar mass nanocelluloses to form nanocellulose papers and found that the best mechanical properties resulted from high molar mass nanofibrils obtained from carboxymethylation pretreatment of wood pulp fibers followed by mechanical disintegration using a high shear resulted in the highest tensile strength (214 MPa) and strainto-failure (10.1\%) (Henriksson et al. 2008). By mixing and drying a TEMPO-oxidized nanocellulose and montmorillonite nanoplatelet dispersion, Wu et al. 2012 prepared a transparent nanocellulose film.The resultant composite film showed a very high tensile strength of $509 \mathrm{MPa}$ and the capacity to resist oxygen permeability (Wu et al. 2012).

In addition to MFCs, BC is an alternative starting material for nanocellulose paper production, when produced, BC naturally forms membranes, so the filtration step can be omitted (Iguchi et al. 2000). BC papers have been obtained with $60 \mu \mathrm{m}$ thick by pressing the $\mathrm{BC}$ hydrogel and exhibited cavities that were collectively one-third of its volume. Afterwards, by infiltrating a liquid resin or dissolved polymer (e.g., epoxy) into these cavities, BC based nanocomposites have been created. Due to the reinforcement of BC, the mechanical strength of the nanocomposite paper was significantly increased, while the transparency remained roughly the same (Yano et al. 2005). 
The mechanical and optical properties of the cellulose nanomaterials make them an interesting material for reinforcing plastics. Cellulose nanomaterials have been reported to improve the performance of, for example, thermosetting resins, starch-based matrices, soy

protein, rubber latex, and poly (lactide) (Yu et al. 2006). The composite applications may be for use as coatings and films, paints, foams, and packaging. CNC can be aligned to produce tunable optical properties, including transparency color changes.

The super water absorbency of cellulose nanomaterials makes them ideal biodegradable water retention filler in incontinence pads and diapers (Larsson et al. 2012).

\subsection{Packaging}

Various research and development activities have been performed by researchers to promote the use of bio-degradable and eco-friendly packaging materials to replace existing conventional packaging materials available in the market, such as conventional plastic or glass packages.

Cellulosic fibers have traditionally been used in packaging for a wide range of food categories such as dry food products, frozen or liquid foods, beverages and fresh foods (Yousefi et al. 2013).

Compared to other packaging applications, industrial packaging applications are slowly evolving with a few large corporations, including Honeywell, Mitsubishi Gas and Chemical, Bayer, Triton Systems and Nanocor which are currently acting as pioneers in CNF based packaging applications (Youssef et al. 2012).

Most challenging aspect of packaging research is to develop and promote the use of renewable and biodegradable "bio-plastic" which can commercially replace petroleum based plastics and thus help in reducing waste disposal problem. However, biopolymers based packaging has relatively poor mechanical and barrier properties than non-biodegradable counterparts which currently limit their industrial use. Various chemical and physical methods have been proposed in past to improve the mechanical and barrier properties of biopolymer based packaging. Researchers currently suggest that the inherent limitations of 
biopolymer-based packaging materials may be overcome through nanocomposite technology (Arora and Padua 2010).

Cellulose nanomaterial-based foams are being studied for packaging applications in order to replace polystyrene-based foams. The advantage of using cellulose nanomaterials instead of wood-based pulp fibers is that the CNF can rein-force the thin cells in the starch foam, replacing a polymer produced from fossil fuel with a renewable material that decreases weight (Svagan et al. 2008).

Starch and chitosan based nanoparticle is used in biopolymer based plastic with special emphasis on cellulose based nanofibers (Venkateshwarlu and Nagalakshmi 2013). According to Youssef et al.(Youssef et al. 2012), there is a possibility to produce packages with stronger mechanical, barrier and thermal properties by adding an appropriate nanoparticle in food packaging. Beside improvement in properties of food packaging nanomaterials will also prevent the invasion of bacteria and microbes into packed food products through packaging. Some examples of antimicrobial immobilization into cellulose nanofibers are shown in Table 2.1.

Liu et al. 2010 also reported that the preparation of composites with nanoscale fillers has been considered a promising method for improving the gas barrier and mechanical properties without affecting transparency for packaging application. Polymers incorporated with clay nanoparticles were among the first polymer based nanocomposites to emerge on the market with improved materials for food packaging and are already used in packaging for carbonated drinks and in thermo-formed containers for industrial purposes (Siqueira et al. 2010).

Polymers with cellulosic fibre/nanoclay based hybrid materials would provide high barrier, short life, easy disposal and environmentally compatible properties for food packaging materials. Active and intelligent packaging includes advances in delayed oxidation and microbial growth rate, and controlled respiration and moisture migration rate. Intelligent packaging also includes time-temperature indicators, ripeness indicators, biosensors and radio frequency identification (Restuccia et al. 2010). 
Table 2.1 Some examples of antimicrobial immobilization into cellulose nanofibers (Rezaei et al. 2015).

\begin{tabular}{ll}
\hline \multicolumn{1}{c}{ Antimicrobial agent } & \multicolumn{1}{c}{ Function } \\
\hline $\begin{array}{l}\text { ZnO incorporated into the cellulose acetate } \\
\text { nanofiber }\end{array}$ & $\begin{array}{l}\text { Exhibited strong antibacterial activity } \\
\text { against the S. aureus, E. Coli and } \\
\text { Citrobacter. }\end{array}$ \\
$\begin{array}{l}\text { Silver nitrate (particle size ranging } \\
\text { from10-20 nm) incorporated into the } \\
\text { cellulose acetate nanofiber. }\end{array}$ & $\begin{array}{l}\text { Very strong antimicrobial activity against } \\
\text { S. aureus, K. pneumoniae, E. coli and P. } \\
\text { aeruginosa. }\end{array}$ \\
$\begin{array}{l}\text { Silver nanoparticles (average size of 21nm) } \\
\text { incorporated into the cellulose acetate }\end{array}$ & $\begin{array}{l}\text { Excellent antibacterial action against } \\
\text { Gram-positive S. aureus and Gram- } \\
\text { nanofiber. }\end{array}$ \\
$\begin{array}{l}\text { Silver nanopartive E. coli, K. pneumonia and P. } \\
\text { aeruginosa. }\end{array}$ \\
$\begin{array}{l}\text { bacterial cellulose nanofibers. } \\
\text { T4 bacteriophage incorporated into } \\
\text { core/shell electrospun fibers of poly } \\
\text { (ethylene oxide) (PEO), cellulose diacetate } \\
\text { (CDA), and their blends. }\end{array}$ & $\begin{array}{l}\text { Strong antimicrobial potential against E. } \\
\text { coli and S. saureus bacteria }\end{array}$ \\
\hline
\end{tabular}

A recent study by Vargas et al. explored the potential of cereal straws (oats, maize, rapeseed, barley and wheat) to be used in biodegradable packaging applications. The process used to obtain cellulose pulp was having high yields. The yield of wheat straw was (70\%) was highest than other cereal trawls (Vargas et al. 2012).

By mixing $\mathrm{CNC}$ with amorphous calcium carbonate (ACC), hard and transparent hybrids with high content of inorganic matter were obtained. Such materials can be of potential interest in packaging and labeling industries (Gebauer et al. 2011).

\subsection{Construction: Soundproofing and thermal insulation}

Over the past years, advancements have been made towards creating cheaper, thinner, more breathable insulating materials with higher R-values. There still is no perfect insulation, but aerogel is one of the top contenders. This new material has the lowest bulk density of any known porous solid, as well as significant insulating qualities. This is in the low-volume category because while aerogel can make a highly insulating material with low energy inputs, it does not re-quire much cellulose nanomaterial. 
Cellulose aerogels can be fabricated from bacterial cellulose, wood/paper pulps, or cellulosic wastes. The aerogels become water-repellent after being treated with silane reagents via a chemical vapor deposition (CVD) method. They show highly porous structures with good flexibility, high stability, and extremely low thermal conductivities. These characteristics make them promising for thermal insulation applications(Duong and Nguyen 2016; Shatkin et al. 2014).

\subsection{Construction: cement composites}

Nanofibrillated cellulose (NFC) has been evaluated as a potential reinforcement for cement mortar composites. Concrete can be reinforced with a combination of cellulose nanomaterials and micro-cellulose fibrils to increase the toughness of an otherwise brittle material. The addition of up to 3\% micro- and nanofibrils in combination increased the fracture energy by more than 50\% relative to the unreinforced material. Benefits include reducing the volume of cement needed, which will lower material and labor costs and result in reduction in associated greenhouse gas emissions (Ardanuy Raso et al. 2012).

\subsection{Structural: Automotive and Aerospace}

NFC can be used as stable, extremely reactive raw materials for technical applications while having the additional advantages over extraction-based products of being renewable, biologically produced, and biodegradable. Such applications include reinforcing (bio-) polymers to create very promising, environmentally safe, lightweight construction materials for the car industry.

$\mathrm{CNC}$ has been incorporated in polyethylene (PE), polypropylene (PP), and biopolymers (PLA and PHA), where it enhances mechanical and barrier properties, as well as abrasion resistance. CNC is envisioned as improving prospects for using bio-plastics in interior automotive parts. Further, the ability to create aerogels and structural foams can result in development of lightweight decorative and interior panels such as the dashboard and door panels dimensional stability, structural strength, thermal resistance, chemical resistance, weight reduction, and electrical conductivity are Typical properties making new efforts underway to develop and apply sustainable nanocomposites that improve structural 
properties for applications in the aerospace industry (Kiziltas et al. 2013; Lyne 2013; RODEANU et al. ; Shatkin et al. 2014).

\subsection{Water purification}

Nanofibrillated cellulose has been used for separation of heavy metal and oil from water (Cervin et al. 2012; Sehaqui et al. 2014). As an example, A highly porous (> 99\%) cellulose nanomaterial aerogel with high structural flexibility and robustness is first formed by freezedrying an aqueous dispersion of the cellulose nanomaterials to achieve a novel type of sponge-like material for the separation of mixed oil and water liquids.The hydrophobic lightweight aerogels are able to selectively absorb oil from water, with a capacity to absorb up to 45 times their own weight in oil. The oil can also be drained from the aerogel, and the aerogel can then be reused for a secondabsorption cycle (Cervin et al. 2012). Additionally, commercial companies are designing, developing and optimizing novel bio-based foams/filters/membranes/adsorbent materials with high and specific selectivity using combinations of cellulose nanomaterials/nano-chitin for decentralized industrial and domestic water treatment. Researchers are developing novel water purification approach combining the physical filtration process and the adsorption process exploring the capability of the cellulose nanomaterials and/or nano-chitin to selectively adsorb, store and desorb contaminants from industrial water and drinking water while passing through a highly porous or permeable membrane (Shatkin et al. 2014).

\subsection{Textiles - clothing}

The development of electrospinning techniques that produce continuous fibers can be applied to develop nanocellulose composite fabrics (Postek et al. 2013).Recently, scientists have developed a highly processed form of cellulose nanomaterials. Once mass produced, this will give engineers stronger, lighter, more durable textile materials to make clothing. Reversible formation and disruption of a cellulose nano-whisker (CNW) percolation network in an elastomeric thermoplastic polyurethane (TPU) matrix has been reported by Zhu et al. which leads to a rapidly switchable shape-memory effect (SME) as a result of temperature change and water immersion. Such rapid switchable water sensitive shape-memory materials 
could broaden the application of CNC in smart textiles, e.g. breathable clothing (Zhu et al. 2012)

\subsection{References}

Aravamudhan A et al. (2013) Cellulose and collagen derived micro-nano structured scaffolds for bone tissue engineering Journal of biomedical nanotechnology 9:719-731

Ardanuy Raso M, Claramunt Blanes J, Arévalo Peces R, Parés Sabatés F, Aracri E, Vidal Lluciá T (2012) Nanofibrillated cellulose (NFC) as a potential reinforcement for high performance cement mortar composites BioResources 7:3883-3894

Arora A, Padua G (2010) Review: nanocomposites in food packaging Journal of Food science 75 (1): 12-21

Auad ML, Contos VS, Nutt S, Aranguren MI, Marcovich NE (2008) Characterization of nanocellulose-reinforced shape memory polyurethanes Polymer International 57:651-659

Auad ML et al. (2011) Polyaniline-modified cellulose nanofibrils as reinforcement of a smart polyurethane Polymer International 60:743-750

Aulin C, Gällstedt M, Lindström T (2010) Oxygen and oil barrier properties of microfibrillated cellulose films and coatings Cellulose 17:559-574

Bäckdahl H, Esguerra M, Delbro D, Risberg B, Gatenholm P (2008) Engineering microporosity in bacterial cellulose scaffolds Journal of tissue engineering and regenerative medicine 2:320-330

Bäckdahl H, Helenius G, Bodin A, Nannmark U, Johansson BR, Risberg B, Gatenholm P (2006) Mechanical properties of bacterial cellulose and interactions with smooth muscle cells Biomaterials 27:2141-2149

Barua S, Das G, Aidew L, Buragohain AK, Karak N (2013) Copper-copper oxide coated nanofibrillar cellulose: a promising biomaterial RSC Advances 3:14997-15004

Barud HS, Regiani T, Marques RF, Lustri WR, Messaddeq Y, Ribeiro SJ (2011) Antimicrobial bacterial cellulose-silver nanoparticles composite membranes Journal of Nanomaterials 2011:10

Bellamkonda RV, Pai SB, Renaud P (2012) Materials for neural interfaces MRS bulletin 37:557-561

Berti FV, Rambo CR, Dias PF, Porto LM (2013) Nanofiber density determines endothelial cell behavior on hydrogel matrix Materials Science and Engineering: C 33:46844691

Bodin A, Ahrenstedt L, Fink H, Brumer H, Risberg B, Gatenholm P (2007) Modification of nanocellulose with a xyloglucan-RGD conjugate enhances adhesion and 
proliferation of endothelial cells: implications for tissue engineering Biomacromolecules 8:3697-3704

Butchosa N, Brown C, Larsson PT, Berglund LA, Bulone V, Zhou Q (2013) Nanocomposites of bacterial cellulose nanofibers and chitin nanocrystals: fabrication, characterization and bactericidal activity Green Chemistry 15:3404-3413

Caldeira E, Piskin E, Granadeiro L, Silva F, Gouveia IC (2013) Biofunctionalization of cellulosic fibres with l-cysteine: Assessment of antibacterial properties and mechanism of action against Staphylococcus aureus and Klebsiella pneumoniae Journal of biotechnology 168:426-435

Capadona JR, Shanmuganathan K, Tyler DJ, Rowan SJ, Weder C (2008) Stimuli-responsive polymer nanocomposites inspired by the sea cucumber dermis Science 319:13701374

Capadona JR, Tyler DJ, Zorman CA, Rowan SJ, Weder C (2012) Mechanically adaptive nanocomposites for neural interfacing MRS bulletin 37:581-589

Capadona JR, Van Den Berg O, Capadona LA, Schroeter M, Rowan SJ, Tyler DJ, Weder C (2007) A versatile approach for the processing of polymer nanocomposites with selfassembled nanofibre templates Nature Nanotechnology 2:765-769

Cassano R, Trombino S, Ferrarelli T, Barone E, Arena V, Mancuso C, Picci N (2010) Synthesis, characterization, and anti-inflammatory activity of diclofenac-bound cotton fibers Biomacromolecules 11:1716-1720

Cassano R, Trombino S, Ferrarelli T, Nicoletta FP, Mauro MV, Giraldi C, Picci N (2013) Hemp fiber (Cannabis sativa L.) derivatives with antibacterial and chelating properties Cellulose 20:547-557

Cervin NT, Aulin C, Larsson PT, Wågberg L (2012) Ultra porous nanocellulose aerogels as separation medium for mixtures of oil/water liquids Cellulose 19:401-410

Chang C-W, Wang M-J (2013) Preparation of microfibrillated cellulose composites for sustained release of h2o2 or o2 for biomedical applications ACS Sustainable Chemistry \& Engineering 1:1129-1134

Chiappone A et al. (2011) Microfibrillated cellulose as reinforcement for Li-ion battery polymer electrolytes with excellent mechanical stability Journal of Power Sources 196:10280-10288

Czaja WK, Young DJ, Kawecki M, Brown RM (2007) The future prospects of microbial cellulose in biomedical applications Biomacromolecules 8:1-12

De Moura MR, Mattoso LH, Zucolotto V (2012) Development of cellulose-based bactericidal nanocomposites containing silver nanoparticles and their use as active food packaging Journal of Food Engineering 109:520-524

Dong H, Snyder JF, Tran DT, Leadore JL (2013) Hydrogel, aerogel and film of cellulose nanofibrils functionalized with silver nanoparticles Carbohydrate Polymers 95:760767 
Dong S, Cho HJ, Lee YW, Roman M (2014) Synthesis and cellular uptake of folic acidconjugated cellulose nanocrystals for cancer targeting Biomacromolecules 15:15601567

Dugan JM, Gough JE, Eichhorn SJ (2010) Directing the morphology and differentiation of skeletal muscle cells using oriented cellulose nanowhiskers Biomacromolecules 11:2498-2504

Duong HM, Nguyen ST (2016) Nanocellulose aerogels as thermal insulation materials. In: Nano and Biotech Based Materials for Energy Building Efficiency. Springer, pp 411427

Edgar CD, Gray DG (2001) Induced circular dichroism of chiral nematic cellulose films Cellulose 8:5-12

Fan L et al. (2013) Preparation, characterization and the effect of carboxymethylated chitosan-cellulose derivatives hydrogels on wound healing Journal of Applied Polymer Science 128:2789-2796

Fernandes SC et al. (2013) Bioinspired antimicrobial and biocompatible bacterial cellulose membranes obtained by surface functionalization with aminoalkyl groups ACS applied materials \& interfaces 5:3290-3297

Filpponen I, Sadeghifar H, Argyropoulos DS (2011) Photoresponsive cellulose nanocrystals Nanomaterials and nanotechnology 1:7

Fu L, Zhang J, Yang G (2013a) Present status and applications of bacterial cellulose-based materials for skin tissue repair Carbohydrate Polymers 92:1432-1442

Fu L et al. (2012) Skin tissue repair materials from bacterial cellulose by a multilayer fermentation method Journal of Materials Chemistry 22:12349-12357

Fu L, Zhou P, Zhang S, Yang G (2013b) Evaluation of bacterial nanocellulose-based uniform wound dressing for large area skin transplantation Materials Science and Engineering: C 33:2995-3000

Fukuya MN, Senoo K, Kotera M, Yoshimoto M, Sakata O (2014) Enhanced oxygen barrier property of poly (ethylene oxide) films crystallite-oriented by adding cellulose single nanofibers Polymer 55:5843-5846

Gao K, Shao Z, Li J, Wang X, Peng X, Wang W, Wang F (2013) Cellulose nanofibergraphene all solid-state flexible supercapacitors Journal of Materials Chemistry A 1:63-67

Gatenholm P, Klemm D (2010) Bacterial nanocellulose as a renewable material for biomedical applications MRS bulletin 35:208-213

Gebauer D, Oliynyk V, Salajkova M, Sort J, Zhou Q, Bergström L, Salazar-Alvarez G (2011) A transparent hybrid of nanocrystalline cellulose and amorphous calcium carbonate nanoparticles Nanoscale 3:3563-3566

Grande CJ, Torres FG, Gomez CM, Bañó MC (2009) Nanocomposites of bacterial cellulose/hydroxyapatite for biomedical applications Acta Biomaterialia 5:16051615 
Harris J, Hess AE, Rowan SJ, Weder C, Zorman CA, Tyler DJ, Capadona JR (2011) In vivo deployment of mechanically adaptive nanocomposites for intracortical microelectrodes Journal of Neural Engineering 8:046010

Helenius G, Bäckdahl H, Bodin A, Nannmark U, Gatenholm P, Risberg B (2006) In vivo biocompatibility of bacterial cellulose Journal of Biomedical Materials Research Part A 76:431-438

Henriksson M, Berglund LA, Isaksson P, Lindstrom T, Nishino T (2008) Cellulose nanopaper structures of high toughness Biomacromolecules 9:1579-1585

Hess AE et al. (2011) Development of a stimuli-responsive polymer nanocomposite toward biologically optimized, MEMS-based neural probes Journal of Micromechanics and Microengineering 21:054009

Hu L et al. (2013) Transparent and conductive paper from nanocellulose fibers Energy \& Environmental Science 6:513-518

Huang J, Zhu H, Chen Y, Preston C, Rohrbach K, Cumings J, Hu L (2013) Highly transparent and flexible nanopaper transistors Acs Nano 7:2106-2113

Iguchi M, Yamanaka S, Budhiono A (2000) Bacterial cellulose-a masterpiece of nature's arts Journal of Materials Science 35:261-270

Jabbour L, Bongiovanni R, Chaussy D, Gerbaldi C, Beneventi D (2013) Cellulose-based Liion batteries: a review Cellulose 20:1523-1545

Jia N, Li S-M, Ma M-G, Sun R-C, Zhu J-F (2012) Hydrothermal fabrication, characterization, and biological activity of cellulose/CaCO 3 bionanocomposites Carbohydrate Polymers 88:179-184

Jorfi M, Roberts MN, Foster EJ, Weder C (2013) Physiologically responsive, mechanically adaptive bio-nanocomposites for biomedical applications ACS applied materials \& interfaces 5:1517-1526

Kalashnikova I, Bizot H, Cathala B, Capron I (2011) New Pickering emulsions stabilized by bacterial cellulose nanocrystals Langmuir 27:7471-7479

Karaaslan MA, Tshabalala MA, Yelle DJ, Buschle-Diller G (2011) Nanoreinforced biocompatible hydrogels from wood hemicelluloses and cellulose whiskers Carbohydrate Polymers 86:192-201

Kettunen M et al. (2011) Photoswitchable superabsorbency based on nanocellulose aerogels Advanced Functional Materials 21:510-517

Kim J-H et al. (2015) Review of nanocellulose for sustainable future materials International Journal of Precision Engineering and Manufacturing-Green Technology 2:197-213

Kiziltas A, Erbas Kiziltas E, Boran S, Gardner DJ Micro-and nanocellulose composites for automotive applications. In: Proceedings of SPE automotive composites conference and exhibition (ACCE), September, 2013. pp 11-13

Klemm D, Heublein B, Fink HP, Bohn A (2005) Cellulose: fascinating biopolymer and sustainable raw material Angewandte Chemie International Edition 44:3358-3393 
Klemm D, Schumann D, Udhardt U, Marsch S (2001) Bacterial synthesized celluloseartificial blood vessels for microsurgery Progress in Polymer Science 26:1561-1603

Kolakovic R, Peltonen L, Laaksonen T, Putkisto K, Laukkanen A, Hirvonen J (2011) Spraydried cellulose nanofibers as novel tablet excipient Aaps Pharmscitech 12:1366-1373

Kumbar S et al. (2011) Novel mechanically competent polysaccharide scaffolds for bone tissue engineering Biomedical Materials 6:065005

Larsson K, Berglund LA, Ankerfors M, Lindström T (2012) Polylactide latex/nanofibrillated cellulose bionanocomposites of high nanofibrillated cellulose content and nanopaper network structure prepared by a papermaking route Journal of Applied Polymer Science 125:2460-2466

Levesque $\mathrm{S}$ et al. (2010) Reactive microgliosis: extracellular $\mu$-calpain and microgliamediated dopaminergic neurotoxicity Brain 133:808-821

Li S-M, Jia N, Ma M-G, Zhang Z, Liu Q-H, Sun R-C (2011) Cellulose-silver nanocomposites: Microwave-assisted synthesis, characterization, their thermal stability, and antimicrobial property Carbohydrate Polymers 86:441-447

Liu H, Yang S, Ni Y (2010) Effect of Pulp Fines on the Dye- Fiber Interactions during the Color-Shading Process Industrial \& Engineering Chemistry Research 49:8544-8549

Lyne B (2013) Market Prospects for NanoCellulose The Royal Institute of Technology, Alberta Biomaterials Development Centre, Edmunton, AB, Canada

Malm CJ, Risberg B, Bodin A, Bäckdahl H, Johansson BR, Gatenholm P, Jeppsson A (2012) Small calibre biosynthetic bacterial cellulose blood vessels: 13-months patency in a sheep model Scandinavian Cardiovascular Journal 46:57-62

Mangalam AP (2008) DNA based artificial nanostructures: directed assembly of cellulose nanocrystals into advanced nanomaterials.

Mucalo MR, Kato K, Yokogawa Y (2009) Phosphorylated, cellulose-based substrates as potential adsorbents for bone morphogenetic proteins in biomedical applications: a protein adsorption screening study using cytochrome $\mathrm{C}$ as a bone morphogenetic protein mimic Colloids and Surfaces B: Biointerfaces 71:52-58

Nagashima K et al. (2014) Cellulose nanofiber paper as an ultra flexible nonvolatile memory Sci Rep 4:5532

Nimeskern L, Ávila HM, Sundberg J, Gatenholm P, Müller R, Stok KS (2013) Mechanical evaluation of bacterial nanocellulose as an implant material for ear cartilage replacement Journal of the Mechanical Behavior of Biomedical Materials 22:12-21

Ninan N, Muthiah M, Park I-K, Elain A, Thomas S, Grohens Y (2013) Pectin/carboxymethyl cellulose/microfibrillated cellulose composite scaffolds for tissue engineering Carbohydrate Polymers 98:877-885

O'brien FJ (2011) Biomaterials \& scaffolds for tissue engineering Materials today 14:88-95

Okahisa Y, Yoshida A, Miyaguchi S, Yano H (2009) Optically transparent wood-cellulose nanocomposite as a base substrate for flexible organic light-emitting diode displays Composites Science and Technology 69:1958-1961 
Peresin MS, Habibi Y, Vesterinen A-H, Rojas OJ, Pawlak JJ, Seppälä JV (2010) Effect of moisture on electrospun nanofiber composites of poly (vinyl alcohol) and cellulose nanocrystals Biomacromolecules 11:2471-2477

Petersen N, Gatenholm P (2011) Bacterial cellulose-based materials and medical devices: current state and perspectives Applied microbiology and biotechnology 91:1277

Postek MT, Moon RJ, Rudie AW, Bilodeau MA (2013) Production and applications of cellulose. Tappi Press. Peachtree Corners,

Potter KA, Jorfi M, Householder KT, Foster EJ, Weder C, Capadona JR (2014) Curcuminreleasing mechanically adaptive intracortical implants improve the proximal neuronal density and blood-brain barrier stability Acta Biomaterialia 10:2209-2222

Pushparaj VL et al. (2007) Flexible energy storage devices based on nanocomposite paper Proceedings of the National Academy of Sciences 104:13574-13577

Puskas JE, Chen Y (2004) Biomedical application of commercial polymers and novel polyisobutylene-based thermoplastic elastomers for soft tissue replacement Biomacromolecules 5:1141-1154

Qin G, Panilaitis BJ, Kaplan ZSDL (2014) A cellulosic responsive "living” membrane Carbohydrate Polymers 100:40-45

Raghavendra GM, Jayaramudu T, Varaprasad K, Ramesh S, Raju KM (2014) Microbial resistant nanocurcumin-gelatin-cellulose fibers for advanced medical applications RSC Advances 4:3494-3501

Rambo C, Recouvreux D, Carminatti C, Pitlovanciv A, Antônio R, Porto L (2008) Template assisted synthesis of porous nanofibrous cellulose membranes for tissue engineering Materials Science and Engineering: C 28:549-554

Restuccia D et al. (2010) New EU regulation aspects and global market of active and intelligent packaging for food industry applications Food Control 21:1425-1435

Rezaei A, Nasirpour A, Fathi M (2015) Application of cellulosic nanofibers in food science using electrospinning and its potential risk Comprehensive Reviews in Food Science and Food Safety 14:269-284

Rodeanu MS, Bastiurea M, Andrei G, Dumitru D. (2013). Properties and applications of nanocellulose polymer composites. A short review. Synthesis 7(11):12

Rodríguez K, Renneckar S, Gatenholm P (2011) Biomimetic calcium phosphate crystal mineralization on electrospun cellulose-based scaffolds ACS applied materials \& interfaces 3:681-689

Salmieri S et al. (2014) Antimicrobial nanocomposite films made of poly (lactic acid)cellulose nanocrystals (PLA-CNC) in food applications: part A-effect of nisin release on the inactivation of Listeria monocytogenes in ham Cellulose 21:1837-1850

Saska S, Teixeira LN, de Oliveira PT, Gaspar AMM, Ribeiro SJL, Messaddeq Y, Marchetto $\mathrm{R}$ (2012) Bacterial cellulose-collagen nanocomposite for bone tissue engineering Journal of Materials Chemistry 22:22102-22112 
Schumann DA et al. (2009) Artificial vascular implants from bacterial cellulose: preliminary results of small arterial substitutes Cellulose 16:877-885

Sehaqui H, de Larraya UP, Liu P, Pfenninger N, Mathew AP, Zimmermann T, Tingaut P (2014) Enhancing adsorption of heavy metal ions onto biobased nanofibers from waste pulp residues for application in wastewater treatment Cellulose 21:2831-2844

Sehaqui H, Liu A, Zhou Q, Berglund LA (2010) Fast preparation procedure for large, flat cellulose and cellulose/inorganic nanopaper structures Biomacromolecules 11:21952198

Shanmuganathan K, Capadona JR, Rowan SJ, Weder C (2009) Stimuli-responsive mechanically adaptive polymer nanocomposites ACS applied materials \& interfaces 2:165-174

Shanmuganathan K, Capadona JR, Rowan SJ, Weder C (2010) Biomimetic mechanically adaptive nanocomposites Progress in polymer science 35:212-222

Shatkin JA, Wegner TH, Bilek E, Cowie J (2014) Market projections of cellulose nanomaterial-enabled products-Part 1: Applications TAPPI J 13:9-16

Shi S, Chen S, Zhang X, Shen W, Li X, Hu W, Wang H (2009) Biomimetic mineralization synthesis of calcium-deficient carbonate-containing hydroxyapatite in a threedimensional network of bacterial cellulose Journal of Chemical Technology and Biotechnology 84:285-290

Shi Z, Li Y, Chen X, Han H, Yang G (2014) Double network bacterial cellulose hydrogel to build a biology-device interface Nanoscale 6:970-977

Siqueira G, Bras J, Dufresne A (2010) Cellulosic bionanocomposites: a review of preparation, properties and applications Polymers 2:728-765

Sun X, Zhang L, Cao Z, Deng Y, Liu L, Fong H, Sun Y (2010) Electrospun composite nanofiber fabrics containing uniformly dispersed antimicrobial agents as an innovative type of polymeric materials with superior antimicrobial efficacy ACS applied materials \& interfaces 2:952-956

Svagan AJ, Samir MA, Berglund LA (2008) Biomimetic foams of high mechanical performance based on nanostructured cell walls reinforced by native cellulose nanofibrils Advanced Materials 20:1263-1269

Svensson A, Nicklasson E, Harrah T, Panilaitis B, Kaplan D, Brittberg M, Gatenholm P (2005) Bacterial cellulose as a potential scaffold for tissue engineering of cartilage Biomaterials 26:419-431

Takehana Y, Yamada A, Tamori M, Motokawa T (2014) Softenin, a novel protein that softens the connective tissue of sea cucumbers through inhibiting interaction between collagen fibrils PloS one 9:e85644

Thavasi V, Singh G, Ramakrishna S (2008) Electrospun nanofibers in energy and environmental applications Energy \& Environmental Science 1:205-221

Tobjörk D, Österbacka R (2011) Paper electronics Advanced Materials 23:1935-1961 
Trotter J et al. (2000) Towards a fibrous composite with dynamically controlled stiffness: lessons from echinoderms. Portland Press Limited,

Ummartyotin S, Juntaro J, Sain M, Manuspiya H (2012) Development of transparent bacterial cellulose nanocomposite film as substrate for flexible organic light emitting diode (OLED) display Industrial Crops and Products 35:92-97

Vargas F, González Z, Sánchez R, Jiménez L, Rodríguez A (2012) Cellulosic pulps of cereal straws as raw material for the manufacture of ecological packaging BioResources 7:4161-4170

Venkateshwarlu G, Nagalakshmi K (2013) Developments in bionanocomposite films: prospects for eco-friendly and smart food packaging Biotechnology and Development:51

Wang J, Gao C, Zhang Y, Wan Y (2010) Preparation and in vitro characterization of BC/PVA hydrogel composite for its potential use as artificial cornea biomaterial Materials Science and Engineering: C 30:214-218

Wang M et al. (2013) Modifying native nanocellulose aerogels with carbon nanotubes for mechanoresponsive conductivity and pressure sensing Advanced Materials 25:24282432

Watanabe K, Eto Y, Takano S, Nakamori S, Shibai H, Yamanaka S (1993) A new bacterial cellulose substrate for mammalian cell culture Cytotechnology 13:107-114

Wei H, Rodriguez K, Renneckar S, Vikesland PJ (2014) Environmental science and engineering applications of nanocellulose-based nanocomposites Environmental Science: Nano 1:302-316

Willgert M, Leijonmarck S, Lindbergh G, Malmström E, Johansson M (2014) Cellulose nanofibril reinforced composite electrolytes for lithium ion battery applications Journal of Materials Chemistry A 2:13556-13564

Wippermann J, Schumann D, Klemm D, Kosmehl H, Salehi-Gelani S, Wahlers T (2009) Preliminary results of small arterial substitute performed with a new cylindrical biomaterial composed of bacterial cellulose European Journal of Vascular and Endovascular Surgery 37:592-596

Wu C-N, Saito T, Fujisawa S, Fukuzumi H, Isogai A (2012) Ultrastrong and high gas-barrier nanocellulose/clay-layered composites Biomacromolecules 13:1927-1932

Wu L, Zhou H, Sun H-J, Zhao Y, Yang X, Cheng SZ, Yang G (2013) Thermoresponsive bacterial cellulose whisker/poly (NIPAM-co-BMA) nanogel complexes: synthesis, characterization, and biological evaluation Biomacromolecules 14:1078-1084

Xiao W, Xu J, Liu X, Hu Q, Huang J (2013) Antibacterial hybrid materials fabricated by nanocoating of microfibril bundles of cellulose substance with titania/chitosan/silvernanoparticle composite films Journal of Materials Chemistry B 1:3477-3485

Yang G, Xie J, Deng Y, Bian Y, Hong F (2012) Hydrothermal synthesis of bacterial cellulose/AgNPs composite: a "green" route for antibacterial application Carbohydrate Polymers 87:2482-2487 
Yang SY, Mahadeva SK, Kim J (2010) Wirelessly driven electro-active paper actuator made with cellulose-polypyrrole-ionic liquid and dipole rectenna Smart Materials and Structures 19:105026

Yano H, Sugiyama J, Nakagaito AN, Nogi M, Matsuura T, Hikita M, Handa K (2005) Optically transparent composites reinforced with networks of bacterial nanofibers Advanced Materials 17:153-155

Yousefi H, Faezipour M, Hedjazi S, Mousavi MM, Azusa Y, Heidari AH (2013) Comparative study of paper and nanopaper properties prepared from bacterial cellulose nanofibers and fibers/ground cellulose nanofibers of canola straw Industrial Crops and Products 43:732-737

Youssef AM, El-Samahy MA, Rehim MHA (2012) Preparation of conductive paper composites based on natural cellulosic fibers for packaging applications Carbohydrate Polymers 89:1027-1032

Yu L, Dean K, Li L (2006) Polymer blends and composites from renewable resources Progress in polymer science 31:576-602

Zhang Z et al. (2011) Thermo-and pH-responsive HPC-g-AA/AA hydrogels for controlled drug delivery applications Polymer 52:676-682

Zheng G, Cui Y, Karabulut E, Wågberg L, Zhu H, Hu L (2013) Nanostructured paper for flexible energy and electronic devices MRS bulletin 38:320-325

Zhou Y, Ding E-Y, Li W-D (2007) Synthesis of TiO 2 nanocubes induced by cellulose nanocrystal (CNC) at low temperature Materials Letters 61:5050-5052

Zhou Y et al. (2014) Efficient recyclable organic solar cells on cellulose nanocrystal substrates with a conducting polymer top electrode deposited by film-transfer lamination Organic Electronics 15:661-666

Zhu $\mathrm{H}$ et al. (2013) Tin anode for sodium-ion batteries using natural wood fiber as a mechanical buffer and electrolyte reservoir Nano letters 13:3093-3100

Zhu Y et al. (2012) Rapidly switchable water-sensitive shape-memory cellulose/elastomer nano-composites Soft Matter 8:2509-2517

Zimmermann KA, LeBlanc JM, Sheets KT, Fox RW, Gatenholm P (2011) Biomimetic design of a bacterial cellulose/hydroxyapatite nanocomposite for bone healing applications Materials Science and Engineering: C 31:43-49 


\title{
CHAPTER 3: NANOFIBRILLATED CELLULOSE FROM APPALACHIAN HARDWOODS LOGGING RESIDUES
}

\begin{abstract}
TEMPO nanofibrillated cellulose (TNFC) from two underutilized Appalachian hardwoods, Northern Red oak (Quercus rubra) and Yellow poplar (Liriodendron tulipifera), was prepared to determine its feasibility to be used as template for antimicrobial metallic copper particles. In addition, a comparison of the TNFC from the two species in terms of their morphological, chemical, thermal and mechanical properties was also performed. The woody biomass was provided in the form of logging residue from Preston County, West Virginia. A traditional kraft process was used to produce the pulp followed by a five-stage bleaching. Bleached pulps were then subjected to a TEMPO-oxidation process using the TEMPO/NaBr$/ \mathrm{NaClO}$ system to facilitate the final mechanical fibrillation process and surface incorporation of metallic copper. The final TNFC diameters for Red oak and Yellow poplar presented similar dimensions, $3.8 \pm 0.74 \mathrm{~nm}$ and $3.6 \pm 0.85 \mathrm{~nm}$, respectively. The TNFC films fabricated from both species exhibited no statistical differences in both the Young's modulus and the final strength properties. Likely, after the TEMPO oxidation process both species exhibited similar carboxyl group content, of approximately $0.8 \mathrm{mmol} / \mathrm{g}$, and both species demonstrated excellent capability to incorporate antimicrobial copper on their surfaces.
\end{abstract}




\author{
Abbreviations \\ NFC Nanofibrillated cellulose \\ TNFC Tempo nanofibrillated cellulose \\ TEMPO 2,2,6,6-tetramethylpiperidine-1-oxyl radical \\ XRDX-ray diffraction \\ R.O Red oak \\ Y.P Yellow poplar \\ TOWP TEMPO oxidized (bleached) wood pulp \\ TEM Transmission electron microscopy \\ SEM Scanning electron microscopy \\ DP Degree of Polymerization
}




\subsection{Introduction}

The production of nanofibrilated cellulose (NFC) from woody biomass has been the subject of intensive investigation in recent years, primarily, due to their unique properties including: non-toxicity, biodegradability, renewability, biocompatibility, high specific surface area, high mechanical performance, and feasibility to be chemically modified (Saito et al. 2007). These properties are opening new opportunities for NFC applications in areas that include the food industry, papermaking, pharmaceutical/medical, and composites/nanocomposites among others (Jin et al. 2013; Pääkkönen et al. 2016).

NFC is nano-sized cellulosic chains with dimensions less than 50nm wide and lengths in the order of several micrometers (Meng et al. 2014; Syverud et al. 2011). NFC can be produced by applying a mechanical treatment such as high-pressure homogenization, microfluidization, grinding and ultra-sonication, among others, to bleached pulps (Pääkkönen et al. 2016). One important disadvantage of any mechanical treatment is their high energy consumption which has been addressed applying a preliminary pretreatment. Enzymatic and/or chemical pretreatment have demonstrated to decrease the energy demand because they will weaken the bonds that hold the cellulosic chains together making it easier to break them into nanocellulose or nanofibrilated cellulose (Siró and Plackett 2010). The chemical pretreatment TEMPO-mediated oxidation process will facilitate the breakup of the fiber network and release the nanofibrils through electrostatic repulsion and osmotic effects.

TEMPO or 2,2,6,6-tetramethylpiperidine-1-oxyl is a highly stable nitroxyl radical which is used extensively in the selective oxidation of primary alcohols to corresponding aldehydes and carboxylic acids. In aqueous environments, TEMPO catalyzes the conversion of carbohydrate primary alcohols to carboxylate (COO-) functional groups in the presence of a primary oxidizing agent e.g. sodium hypochlorite $(\mathrm{NaOCl})$. In particular, wood fibers can be converted to individual nanofibers 3-4 nm wide with several microns length by TEMPOmediated oxidation and successive mild disintegration in water (Isogai et al. 2011; Rodionova et al. 2012). During this reaction significant amounts of C6 carboxylate groups are selectively formed on each cellulose microfibril surface with minimum changes of the original crystallinity of the cellulosic material. The negative charged carboxylate groups 
introduced by a TEMPO-mediated oxidation will facilitate the subsequent fibrillation process due to the electrostatic forces created among microfibrils (Meng et al. 2014).

To date, some studies have compared NFC characteristics from softwood and hardwood biomass sources (Syverud et al. 2011; Besbes et al. 2011; Brodin et al. 2013; Rodionova et al. 2013; Stelte and Sanadi 2009; Zimmermann et al. 2010). Part of the available information has been focused on the evaluation of the final NFC morphology using only a mechanical fibrillation treatment (Stelte and Sanadi 2009; Zimmermann et al. 2010); the other part has been focused not only in the final NFC morphology but also in its carboxylic acid availability when a preliminary TEMPO-mediated oxidation process is applied to facilitate the mechanical fibrillation (Syverud et al. 2011; Besbes et al. 2011; Brodin et al. 2013; Rodionova et al. 2013). To the knowledge of the authors there is no previous or ongoing research regarding to the use of the modified nanofibrillated surfaces to attach antimicrobial metal particles in addition to the research performed so far at West Virginia University, where we demonstrated that copper nanoparticles can be effectively synthesized on softwoods TEMPO nanofibrillated cellulose (TNFC) (Jiang et al. 2016). Likewise, the effectiveness of this TNFC-supported copper in terms of antimicrobial performance and controlled release from thermoplastic films has been also demonstrated previously (Jiang et al. 2016; Zhong et al. 2015a).

Currently, in the Appalachian region there is a vast amount of low-value, low quality hardwood that can be used as feedstock for novel bio-products. West Virginia is the third most heavily forested state in the nation. The harvesting process yields approximately 2.41 million dry tons of wood residues annually, including 1.34 million dry tones logging residue of which Red oak is the predominant specie, followed by Yellow-poplar and maple species (Wu et al. 2010). Even though these species constitute a potential source for NFC production, to the extent of the authors' knowledge, no study in NFC preparation from any of the mentioned hardwood species has been performed to date. Only one study have been reported in West Virginia regarding to the use of Appalachian underutilized hardwoods as raw material for nanocrystalline cellulose (NCC) production (Jacobson PM 2011). Consequently, the two hardwood species, Red oak (Quercusrubra) and Yellow poplar (Liriodendron 
tulipifera) which possess similar chemical composition (Jin et al. 2013), but different anatomical properties are of interest to evaluate their properties at a nanoscale. Red oak is a ring porous hardwood with prominent and conspicuous rays and thick-walled latewood vessels. Yellow poplar is a diffuse porous hardwood, with small and solitary pores and fine rays.

The goals of this study therefore include: (i) to evaluate the feasibility of obtaining cellulose nanofibers from highly available underutilized logging residues from Red oak and Yellow poplar; (ii) to compare their changes in chemical composition, morphology, carboxyl content, thermal and mechanical properties after TEMPO mediated oxidation; and (iii) to evaluate TNFCs feasibility to synthesize metallic copper for further antimicrobial applications.

\subsection{Materials and Methods}

\subsubsection{Materials}

Northern Red oak (Quercus rubra)(R.O) and Yellow poplar (Liriodendron tulipifera)(Y.P) samples were collected in 2014 at WVU Research Forest, Preston County, West Virginia, in the form of logging residue. The samples were chipped and screened. The fraction retained between $1 / 2$ inch and $3 / 8$ inch were used for pulping.

\subsubsection{Methods}

\subsubsection{Pulping}

Small screened samples of R.O and Y.P were oven dried to determine the moisture content as $12 \%$.kraft pulp from the oven dried material was produced using a M/K System 6-L laboratory digester. The conditions for the pulping process are presented in Table 3.1. All pulping chemical reagents, including effective alkali, $\mathrm{EA}$, $\left(\mathrm{NaOH}+1 / 2 \mathrm{Na}_{2} \mathrm{~S}\right)$, were expressed in terms of $\mathrm{Na}_{2} \mathrm{O}$. Sulfidity was expressed as a percentage of the EA, and the expected $\mathrm{H}$-factor was 750 . The samples were subjected to the maximum temperature of $165^{\circ} \mathrm{C}$ for $50 \mathrm{~min}$, after which the temperature was reduced rapidly. The final pulp was 
disintegrated by vigorous agitation, screened on a Valley flat screen (0.008 inch slot), washed, and then collected in a 200 mesh screen box. The screened wet samples were used for the next step (bleaching) while some small samples were oven and air dried for different analysis. The oven dried pulps were characterized in terms of their lignin content via Kappa number determination. The Kappa numbers of the two species were determined by measuring the consumption of potassium permanganate according to T $236 \mathrm{~cm}-85$ standard method. The percentage of lignin was estimated using the following equation:

Lignin $(\%)=$ Kappa number $\times 0.15 \quad$ Eq.1

\subsubsection{Bleaching}

A five-stage bleaching sequence $\mathrm{DEpDEpD}$ was performed, where $\mathrm{D}$ denotes Chlorine dioxide and Ep represents peroxide reinforced alkaline extraction. The pulps were thoroughly washed between stages. After the completion of all stages, the fibers were washed and oven dried. Table 3.2 shows the conditions of the bleaching processes.

\subsubsection{TEMPO-Mediated Oxidation}

Approximately $30 \mathrm{~g}$ of oven dry basis bleached pulp was added into $3 \mathrm{~L}$ of water that contained TEMPO ( $0.016 \mathrm{~g}$ per $\mathrm{g}$ pulp) and sodium bromide $(0.1 \mathrm{~g} / \mathrm{g}$ pulp). The reaction was started by adding $\mathrm{NaClO}$ solution (5.0 mmol NaClO per g of pulp) with stirring at $500 \mathrm{rpm}$ at room temperature. The $\mathrm{pH}$ was maintained at 10 by adding $0.5 \mathrm{M} \mathrm{NaOH}$ using a $\mathrm{pH}$ stat until no $\mathrm{NaOH}$ consumption was observed. Additional $\mathrm{NaClO}$ was added into each sample as long as no changes in $\mathrm{pH}$ were observed. The TEMPO-oxidized cellulose was thoroughly washed with water by filtration and stored at $4{ }^{\circ} \mathrm{C}$ before homogenization[1]. The yield has been measured based on weight of dried final material and weight of diluted samples which results in approximately 90\% for both TOWP samples.

Samples of each specie were air dried, then used to characterize the degree of polymerization (DP) and carboxylic acid group determination. The rest of the TEMPO-oxidized sample was diluted to $1 \%$ solid content, before homogenization. Mechanical fibrillation was performed by passing the samples twice through an M-110EH-30 Microfluidizer (Microfluidics, 
Newton, MA). In the first step, the material passed through a $200 \mu \mathrm{m}$ chamber and in the second step the suspension passed through 200- and 87- $\mu \mathrm{m}$ chambers in series.

\subsubsection{Chemical analysis}

Samples from kraft and bleached pulps were dried, and ground in a Willey mill to pass a 20 mesh screen (model No.2, Arthur H. Thomasco). These samples along with untreated samples were used for the chemical composition analyses that included Klason lignin, acid soluble lignin, hemicellulose and cellulose determination. High performance anion exchange chromatography (Dionex ICS-3000 system) and the procedure developed by Davis MW (1998) were used for the carbohydrate determination. Lignin was determined based on the amount of sulfuric acid-insoluble Klason lignin, in accordance with the standard of the Technical association of pulp and paper industry (TAPPI) TAPPI standards-T222 om 88. The results are presented in Table 3.3.All the analyses, with the exception of acid soluble lignin, were performed in duplicated.Error! Reference source not found.

\subsubsection{Determination of intrinsic viscosity and degree of polymerization}

Viscosity of the resulting nanofibrillated cellulose was measured according to TAPPI Standard Method T230 om-99. Oven dried cellulosic solids of $0.1 \mathrm{~g}$ were dispersed in 10 $\mathrm{mL}$ distilled water and then added to $10 \mathrm{~mL}$ of $1 \mathrm{M}$ cupriethylene diamine solution $(0.5 \%$ solid concentration). The viscosity of the resultant solution was determined with a capillary viscometer. The degree of polymerization (DP) was estimated by the following equation:

$\mathrm{DP}=120 \times[\eta]^{1.11}$

Where $\eta$ is the measured viscosity; the results are presented in Table 3.4.

\subsubsection{Carboxyl content of the pulp}

Determination of carboxyl content of the samples before and after the TEMPO-mediated oxidation process was performed according to T 237 om-88, modified as indicated below to account for the high acid content of the fiber with some reagent concentration modifications. The pulps were converted to the acid form by soaking them in $0.01 \mathrm{M} \mathrm{HCl}$ and then washing with deionized water. This acidified pulp was reacted with a standardized $0.1 \mathrm{M} \mathrm{NaHCO} 3$ with $0.25 \mathrm{M} \mathrm{NaCl}$ solution. The amount of residual $\mathrm{NaHCO}_{3}$ was determined by titration with 
$0.1 \mathrm{M} \mathrm{HCl}$, to a methyl red endpoint. The carboxyl content was calculated in mili-equivalents (meq) per $100 \mathrm{~g}$ of oven-dry pulp and then converted to $\mathrm{mmol} / \mathrm{g}$ and the results are presented in Table 3.5.

\subsubsection{Fourier transform infra-red spectroscopy (FTIR)}

Fourier transform infrared spectra were recorded for the raw material, bleached pulps, TEMPO-oxidized pulps and the final TEMPO-nanofibrillated cellulose (TNFC)samples using a Perkin-Elmer Spectrum 2000 FTIR spectrometer. The samples were analyzed from 600 to $4,000 \mathrm{~cm}^{-1}$. The spectrum was obtained from dry samples using 16 scans at a $4 \mathrm{~cm}^{-1}$ resolution and a $1 \mathrm{~cm}^{-1}$ interval at room temperature. The results are presented in Figure 3.1.

\subsubsection{X-ray diffraction (XRD) characterization}

$\mathrm{X}$-ray diffraction (XRD) analysis was performed on the raw material, TEMPO-oxidized pulps, and the final TEMPO nanofibrillated cellulose (TNFC) samples. The analysis was performed on a X-Ray diffractometer (PANalyticalX'Pert Pro XRD) in the range of $10^{\circ}$ to $40^{\circ} 2$. $\mathrm{Cu}-\mathrm{k} \alpha 18047.2 \mathrm{eV}$ source and a maximum X-Ray power of $45 \mathrm{kV}$ and $40 \mathrm{~mA}$ were used. The crystallinity index(CI) after the different treatments was determined using the peak height method and calculated by the Segal empirical method (Segal et al. 1959) after subtraction of the background signal:

$$
\mathrm{CI}(\%)=\frac{\mathrm{I} 200-\mathrm{Iam}}{\mathrm{I} 200} \times 100 \% \quad \text { Eq.3 }
$$

Where $\mathrm{I}_{200}$ is the peak intensity corresponding to crystalline cellulose I at $2 \theta=22.6^{\circ}$ and Iam the intensity minimum between the 200 and 110 peaks $\left(2 \theta=18^{\circ}\right)$

The results are presented in Table 3.6 and Figure 3.2.

\subsubsection{Thermogravimetric analysis (TGA)}

Thermogravimetric analysis was performed for the raw material and the final TEMPOnanofibrillated cellulose (TNFC) using 4-6 mg sample. The data was obtained using a TA Q50 thermogravimetric analyzer (Delaware, USA). The temperature was set from $25{ }^{\circ} \mathrm{C}$ to 
$600{ }^{\circ} \mathrm{C}$ at a heating rate of $10{ }^{\circ} \mathrm{C} / \mathrm{min}$ under a nitrogen atmosphere at a flow rate of 20 $\mathrm{mL} / \mathrm{min}$. The results of this analysis are presented in Table 3.7 and and Figure 3.3.

\subsubsection{Scanning electron microscopy (SEM)}

Scanning electron microscopy (SEM) was used to evaluate the effect of mechanical fibrillation on the samples morphology. TEMPO oxidized wood pulp (TOWP) and TEMPO nanofibrillated cellulose (TNFC) were freeze dried after a preliminary solvent exchange process using ethanol and tert-butanol[20]. Freeze drying was conducted on a VirTis Genesis Freeze Dryer (Warminster, PA, USA) at $-40{ }^{\circ} \mathrm{C}$ for 1 week. The morphological analyses of the samples were performed using a Hitachi S-4700 FESEM-EDX. Before the FESEM-EDX analyses, all the samples were coated with gold-palladium using a sputtering process to provide adequate conductivity. The diameter of different samples after bleaching and TEMPO oxidation were measured and calculated using the ImageJ Manipulation Program (Ferreira and Rasband 2012) for at least 30 fiber for each sample. Results are presented in Figure 3.4.

\subsubsection{Transmission electron microscope (TEM)}

For TEM analysis TNFC samples of R.O and Y.P were used as their original undiluted suspension form. Undiluted TNFC suspensions were deposited on a glow-discharged copper grid with formvar and carbon film (400 mesh) for 5 min, and then rinsed thoroughly using a $1 \%$ aqueous uranyl acetate stain followed by blotting dry. Samples were imaged using Philips CM-100 TEM (Philips/FEI Corporation, Eindhoven, Holland) operated at $100 \mathrm{kV}$,

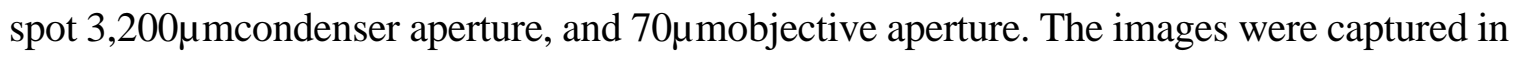
digital form directly on the microscope using GatanOrius SC200-1 2Mpixel CCD camera (Gatan, Inc. Pleasanton, CA) with 19k, 46k and 92k magnification. The diameter of different NFCs were measured and calculated from TEM images using the ImageJ Manipulation Program (Ferreira and Rasband 2012) for at least 50 nanofibers in each sample. The results are presented in Figure 3.5. 


\subsubsection{Preparation and characterization of TNFC films}

TNFC hydrogel films were prepared by filtering TNFC suspensions which were dried afterward. The filtration was performed by first diluting the TNFC suspension to $0.2 \%$ solid by weight with reverse-osmosis-treated water. Following the dilution, the suspensions were filtered using a 142-mm Millipore ultrafiltration system (Millipore Corporation, Billerica, MA, USA) under 0.55 MPa of air pressure. Omnipore TM filter membranes with a pore size of 0.1 $\mu \mathrm{m}$ (JVWP14225, JV, Millipore Corporation, USA) were supported on filter paper in the ultrafiltration system. The wet films (hydrogels) were peeled from the membrane, processed, stacked, and placed between an assembly of waxy coated papers, absorbent blotter paper, and two metal caul plates. The assembly was air-dried at room temperature for $24 \mathrm{~h}$ and then oven-dried at $60^{\circ} \mathrm{C}$ for $8 \mathrm{~h}$ under a load of approximately $250 \mathrm{~N}$. The blotter and filter papers were replaced several times over the first $24 \mathrm{~h}$ to minimize wrinkling of the films (Quing et al. 2015).

The tensile strength of different NFC films were tested using an Instron 5865 universal material testing apparatus (Instron Engineering Corporation, MA, USA) with a $500 \mathrm{~N}$ load cell, according to ASTM D638-10. The specimens were cut according to ASTM D638-10 type V dog bone shape using a special die (Qualitest, FL, USA) and were subsequently conditioned at $50 \% \mathrm{RH}$ and $23^{\circ} \mathrm{C}$ for at least 1 week prior to testing, also at $50 \% \mathrm{RH}$ and $23^{\circ} \mathrm{C}$. The specimens were pre-loaded with a $5 \mathrm{~N}$ force to remove slack and the tests were performed with a crosshead speed of $1 \mathrm{~mm} / \mathrm{min}$. At least 6 specimens were tested for each sample. A LX 500 laser extensometer (MTS Systems Corporation, MN, USA) was used to determine the displacement with sampling frequency of $10 \mathrm{~Hz}$. The laser recorded the displacement between two strips of reflective tape initially placed $8 \mathrm{~mm}$ apart on the neckeddown region of the dog-bone specimens. Strain was calculated from the displacement and initial gage length (Quing et al. 2013). The stress-strain curves were fit to a hyperbolic tangent and the tensile modulus was taken as the slope of the fit curve at zero strain. The statistical significance was determined using a two-tailed unpaired Student's t-test and pvalue $<0.05$ was considered statistically significant. The maximum tensile strength and the corresponding tensile modulus are presented in Figure 3.6. 


\subsubsection{Preparation of bybrid TNFC and copper nanoparticles}

The hybrids TNFC-metallic copper particles were prepared according to Jiang et al. 2016[14] and Zhong et al. 2015a) (Zhong et al. 2015a). Upon dispersing the hybrid materials into deionized water, they were dried using a preliminary solvent exchange process with ethanol/tert-butanol and then freeze dried as described by Zhong et al. 2015b [20].

\subsubsection{Copper determination on TNFC from Red oak and Yellow poplar}

Copper determination of TNFC-metallic copper particles was conducted using Energy Dispersive X-ray (EDX) analysis and the results are presented in Table 3.9. The morphological analysis of the TNFC-metallic copper particles from Red oak and Yellow poplar were performed using a Hitachi S-4700 FE-SEM; the samples were previously coated with gold-palladium using a sputtering process to provide adequate conductivity. An example of the final morphology of these samples with the presence of copper crystals is presented in Figure 3.7.

\subsection{Results and Discussion}

\subsubsection{Chemical analysis}

The content of the primary constituents (cellulose, hemicellulose and lignin) of the untreated woody samples and bleached pulps are presented in Table 3.3. According to these results, bleached samples of YP contained slightly more cellulose content and less hemicellulose than R.O. For RO and YP, carboxylic content were nearly identical (Table 3.5), and might be one reason indicating that the difference in hemicellulose content between two samples is negligible to effect on carboxyl content. It has also been reported that high content of hemicelluloses facilitates the release of nanofibrils during the mechanical treatment of the pulp (Iwamoto et al. 2008), however as mentioned above, the difference in hemicellulose content in RO and YP does not look significant based on results of carboxyl contents.

In terms of lignin, no differences between R.O and Y.P were determined for both, untreated and bleached pulps. The kappa number (KN) of the kraft pulp was 13.25 and 20 giving the 
lignin percentage of 1.72 and 2.6 for R.O and Y.P after the kraft pulping, respectively. The kappa number varies according to the wood species and delignification procedure during pulping (Li et al. 2002).

\subsubsection{Degree of polymerization (DP)}

The degree of polymerization as a function of the viscosity of bleached and TEMPOoxidized wood pulp (TOWP) from R.O and Y.P is presented in Table 3.4. The viscosity is an indirect measurement of the cellulose polymer chain length and can be used to indicate chemical degradation of the pulps due to chemical or mechanical treatments.

The viscosity and correspondent DP result are higher for R.O bleached and TOWP samples. This result might be related to the previous difference in terms of the hemicellulose content. The TEMPO-mediated oxidation and especially the use of $\mathrm{NaClO}$ inevitably lead to depolymerization of the starting material in both samples of R.O and Y.P, but showed a slight reduction in Y.P samples.

\subsubsection{Carboxyl content}

The carboxylic group content was determined forR.O. and Y.P. bleached pulps and for the R.O. and Y.P.TEMPO-oxidized samples. As presented in Table 3.5 the carboxylic group increased from $0.05-0.07 \mathrm{mmol} / \mathrm{g}$ for the bleached pulps to $0.80-0.82 \mathrm{mmol} / \mathrm{g}$ after the

oxidation process. The increased in the carboxylic content after the TEMPO-mediated oxidation process was similar for both hardwood species.

\subsubsection{FTIR spectroscopy analysis}

The analysis of functional groups through FTIR is presented in Figure 3.1 a) for R.O. and 1b) for Y.P., where (I) corresponds to the initial woody raw material; (II) are the bleached samples; (III) are the TEMPO-oxidized samples (TOWP) and (IV)corresponds to the TEMPO nanofibrillated material (TNFC).

The absorption peaks at $3300 \mathrm{~cm}^{-1}$ and $2920-2800 \mathrm{~cm}^{-1}$ were attributed to of hydrogen bonded hydroxyl $(\mathrm{OH})$ groups of cellulose and absorbed water, and aliphatic saturated $\mathrm{C}-\mathrm{H}$ 
stretching vibration, respectively (Chen et al. 2011; Morais et al. 2013; Poddar et al. 2015; Wicaksono et al. 2013; Wulandari et al. 2016). These peaks increased in intensity after bleaching and Tempo-oxidation.

The bands present at 1730 and $1739 \mathrm{~cm}^{-1}$ which are possible to observe only for the untreated raw material for both R.O and Y.P can be attributed to $\mathrm{C}=\mathrm{O}$ stretching of the acetyl and uronic ester groups of hemicellulose or the ester linkage of carboxylic groups of ferulic and p-coumaric acids of lignin and/or hemicellulose (Alemdar and Sain 2018; Sun et al. 2005). This band was no longer present in the FTIR spectra of R.O and Y.P after bleaching and subsequent TEMPO oxidation treatments. The disappearance of this band is largely due to the removal of hemicellulose and lignin from R.O and Y.P fibers during the chemical extraction (Alemdar and Sain 2018; Jonoobi et al. 2009). The peaks at 1,509-1,504 $\mathrm{cm}^{-1}$ in the spectrums of raw samples of R.O and Y.P represent the $-\mathrm{C}=\mathrm{C}-$ stretch of the aromatic rings of lignin (Sain and Panthapulakkal 2006; Le Troedec 2008). The lower amount of this peak in the treated samples is attributed to the removal of lignin during chemical procedures.

Two strong absorption bands at around 1638-1600 and 1412-1402 $\mathrm{cm}^{-1}$, which correspond to carbonyl groups $-\mathrm{C}=\mathrm{O}$, are present in both spectra in response to the TEMPO-mediated oxidation process. As expected after the TEMPO oxidation process hydroxyl groups at the C6 position of cellulose molecules were converted to sodium carboxylate. The intensity of $\mathrm{C}=\mathrm{O}$ peaks for both hardwood species present the following trend TNFC=TOWP $>$ bleached $>$ raw material individually, whereas, comparing R.O and Y.P graphs these areas have shown same intensity.

The peaks observed in the spectra of the fibers around $1225 \mathrm{~cm}^{-1}$ is due to the C-O stretching of the aryl group in lignin (Le Troedec 2008). Disappearance of this peak from the spectra of the bleached pulp fibers as well as from those of the nanofibers is explained by the removal of lignin due to chemical treatments. 
The stretching of $\mathrm{C}-\mathrm{O}$ and $\mathrm{O}-\mathrm{H}$ and bending vibration of the $\mathrm{C}-\mathrm{O}-\mathrm{C}$ pyranose ring in cellulose molecules (Lani et al. 2014; Liu et al. 2016) can be observed as peaks around 1070$1010 \mathrm{~cm}^{-1}$ for all samples. The peak at $1029 \mathrm{~cm}^{-1}$ for both hardwood species seems to be much higher for TNFC in following the trend TNFC> TOWP>bleached $>$ raw material in R.O and Y.P meaning more carbohydrate content after treatment.

To summarize, the typical absorption band that appears in R.O and Y.P were similar, and there were no significant differences between them after different treatments.

\subsubsection{Crystallinity determination}

The XRD patterns of the raw material, oxidized samples by TEMPO (TOWP) and nanofibrillated cellulose (TNFC) from Red oak and Yellow poplar are presented in Figure 3.2 a) and 2b) respectively. The resultant Crystallinity index for those samples is displayed in Table 3.6.

All samples had diffraction peaks at $2 \theta=16.5^{\circ}$ and $22.5^{\circ}$, which is considered to represent the typical cellulose I pattern (Nishiyama et al. 2002). The crystallinity index (CI) of the original samples was 37 and $34.4 \%$ for R.O and YP, respectively. After oxidation, the apparent crystallinity of samples increased to 61.7 and 59.8\% in R.O and Y.P, respectively owing to the removal of hemicellulose and lignin. This increase was expected, but not that significant. Some artifacts in the measurement of CI might be affecting the results, but since the increase was similar for both species, the values were accepted. The crystallinity went through significant reduction upon the homogenization process to a degree of 39\% in TNFC species of R.O and Y.P, respectively. Mechanical fibrillation appears to affect importantly the final crystallinity of TNFC samples, but once again, caution must be considered here because of possible artifacts associated with the measurements of crystallinity of TEMPO oxidized pulps. Some researchers determined a reduction in crystallinity after a high level homogenization of TEMPO-oxidized pulps due to either breakage or peeling-off of the cellulose (Besbes et al. 2011; Wang et al. 2012; Zimmermann et al. 2010). Considering that even though differences in the crystallinity index after the TEMPOoxidation stage and after 
the fibrillation process were observed, the differences were similarbetween the two hardwood species in study.

\subsubsection{Thermal gravimetric analysis}

Thermogravimetric (TGA) and derivative thermogravimetric (DTG) analyses were performed to compare the thermal degradation characteristics of the untreated and chemomechanical treated R.O and Y.P. Details of the major decomposition temperatures and the corresponding percentage of weight losses are summarized in Table 3.7. The thermal behaviour of lignocellulosic materials depends on their chemical composition, structure, degree of crystallinity and the molecular interactions between the different macromolecules (Maiti et al. 2013). Between the temperatures of $150-500^{\circ} \mathrm{C}$, the degradation process begins in the cellulose, hemicellulose and the associated linked water.

In the TGA curve represented by weight (\%) (Figure 3.3 (a)), thermal degradation of untreated raw samples begins at temperatures 304.5 and $312{ }^{\circ} \mathrm{C}$ (Tonset), whereas in TEMPO oxidized NFCs the Tonset began at 224 and $225^{\circ} \mathrm{C}$ for R.O and Y.P, respectively. In the DTG curve represented by deriv. weight $\left(\% /{ }^{\circ} \mathrm{C}\right.$ ) (Figure 3.3 (b)), both TEMPO-oxidized and untreated samples showed a prominent pyrolysis process, with a clear double steps degradation for TNFC samples (stage I and ॥). The maximum weight loss was observed in the temperature range from $230-290{ }^{\circ} \mathrm{C}$ and $285-366^{\circ} \mathrm{C}$, respectively.

As shown in the DTG curves in Figure 3.3 (b) the untreated R.O showed a small broadening or shoulder at $285^{\circ} \mathrm{C}$ on the left side of the main peak (366 ${ }^{\circ} \mathrm{C}$ ), which may have been due to the decomposition of hemicellulose as hemicelluloses has lower thermal stability than lignin and cellulose (Chirayil et al. 2014).In the case of the untreated Y.P, the DTG curve showed a less sharp peak on the left side of the main peak, which may be explained by the lower content of hemicellulose. This probably resulted in better thermal properties for the untreated Y.P than R.O (304.5 versus 312 in Table 3.7 for raw R.O and raw Y.P, respectively)since the decomposition of hemicellulose begins at much lower temperatures, compared to cellulose (Kabir et al. 2013). The final major decomposition peak observed at 
the high temperature range $\left(300-400^{\circ} \mathrm{C}\right)$ is accounted for the pyrolysis of cellulose. This peak was higher in Y.P compared to R.O in both raw fibers and TNFC samples (stage II of Table 3.7) and might be explain by the fact that Y.P showed a higher cellulose content than R.O by carbohydrate measurement (Table 3.7). As expected, the degradation temperature of TEMPO oxidized NFCs for both R.O and Y.P, were lower than those of untreated samples, because the carboxylic acid groups initiate thermal decomposition at lower temperatures (Deepa 2015). These results are consistent with the results obtained from FTIR and XRD measurements. As presented in Table 3.7, the char residues (CY\%) from TNFC samples were higher than that of raw samples. The large amount of char residue form TEMPO oxidized NFCs is due to the high sodium ion which will hold carbonate, so the high sodium content after neutralization results in a high ash (char) content in of TEMPO treated pulps (TOWP).In summary, based on TGA analysis, TNFC from YP and RO have similar thermal stability.

\subsubsection{Morphology of the Treated and Untreated samples}

Figure 3.4 (a) and (b) are SEM images of bleached samples of R.O and Y.P, respectively. Before the analysis the samples were dried in a conventional laboratory oven at $103^{\circ} \mathrm{C} \pm 2^{\circ}$ C overnight. The average in diameter for R.O fiber was $9.16 \pm 1.06 \mu \mathrm{m}$ and $15.5 \pm 1.04 \mu \mathrm{m}$ for Y.P. According to the SEM images.

Figure 3.4 (c) and (d) represent SEM images of TEMPO oxidized fibers (TOWP) of R.O, and Y.P, respectively. Before the analysis the samples were dried in a conventional laboratory oven at $103^{\circ} \mathrm{C} \pm 2^{\circ} \mathrm{C}$ overnight. The resultant average in diameter for TOWP of R.O is of $7.99 \pm 1.75 \mu \mathrm{m}$ and $17.69 \pm 2.12 \mu \mathrm{m}$ for TOWP of Y.P. The TEMPO oxidation process has not affected the dry fiber diameters;

Figure 3.4 (e) and (f) are SEM images of TEMPO nanofibrillated cellulose (TNFC) from R.O and Y.P, respectively. Before the analysis the samples were dried using a solvent exchange process previous a freeze drying stage (Zhong et al. 2015b). The preparation method aims to produce a material with high specific surface area. As presented in both images a fibrillar and porous network structure resulted for both, Red oak and Yellow poplar 
species. The magnification for these samples was different compared to the previous ones, to highlight the high porous structure resultant for both species after their mechanical fibrillation and drying processes.

In Figure 3.5 (a) and (b) are presented TEM images of TEMPO nanofibrillated cellulose (TNFC) from R.O and Y.P, respectively. As mentioned earlier, the diameter of these TNFCs were measured from TEM images using the Imagej Manipulation Program (Ferreira and Rasband 2012). The average diameter for TNFC from R.O resulted of $3.8 \pm 0.74 \mathrm{~nm}$ and of $3.6 \pm 0.85 \mathrm{~nm}$ for TNFC from Y.P. The maximum diameter was 5-6 $\mathrm{nm}$ with $6 \%$ distribution in both species.

The distribution of diameter percentages of nanofibrils are presented in Figure 3.5 (c) and (d) for TNFC R.O and TNFC Y.P, respectively. This information indicates that there are $10 \%$ more nanofibrils with diameters in the range of 3.0-4.0 nm for R.O compared with Y.P, and also 10\% more nanofibrils with diameters in the range of 5.0-6.0 nm for R.O compared to Y.P. No nanofibrils with diameters in the range 1.0-2.0 nm were found for R.O. In the nanofibril diameter range between 2.0-3.0 nm Y.P resulted $14.5 \%$ higher than R.O. The introduction of negative carboxyl groups on nanofibril surface generated strong repulsive forces, which resulted in separated individual nanofibrils with narrow diameter distribution (Saito et al. 2009).

\subsubsection{Mechanical properties of NFC films}

TNFC films of R.O and Y.P were produced and their mechanical properties were measured. Ten randomly chosen films were used for these measurements. The average film thickness was 127 micrometer for both NFC samples.

The tensile modulus (modulus of elasticity or MOE) of the various NFC films was calculated based on the initial linear region of the stress-strain curve and is shown in Figure 3.6(a). Y.P and R.O showed to be very close in MOE (12.35 \pm 1.02 GPa versus $13.6 \pm 2.09)$ with no significant p-value. Previous studies by other groups showed that tensile modulus strongly depends on the crystallinity of nanofibrils as (Fukuzumi et al. 2013; Qing et al. 2013; Retegi 
A. 2010). The results of MOE in both species was in accordance with XRD analysis by which both TNFC samples showed close range of crystallinity after homogenization (Table 3.6). The strain at break for the various NFC films also demonstrated no significant difference between films of RO $(7.75 \% \pm 1.4)$ compared to Y.P $(6.41 \% \pm 2.04)$ (Figure 3.6 (b) and Table 3.8).

The maximum tensile stress (strength) of films prepared from different NFCs is shown in Figure 3.6 (c) and Table 3.8. It can be seen the TEMPO films from Y.P had almost the same mechanical strength with a small increase of approximately $135.15 \pm 14.2 \mathrm{MPa}$ compared to R.O (129.8 $\pm 16.36 \mathrm{MPa}$ ). This difference is not significant according to the t-test (p-value> than 0.05)

Henriksson et al. (2008) pointed out that the tensile strength, toughness, and strain to failure of nanofiber films correlate with the average molar mass of the nanofibers. Qing et al.(2013) reported similar results from Eucalyptus TNFC samples, showing higher tensile strength of approximately 220 MPa caused mainly because of the higher DP of the oxidized cellulose, while the crystallinity of their TNFC samples were in the range of our samples. Saito et al. (2009) also reported that TEMPO/ $\mathrm{NaClO} / \mathrm{NaClO}_{2}$ system provides $\mathrm{CNF}$ with up to approximately 1.5 times higher tensile strength (312 MPa) compared to those CNF from TEMPO/NaBr/NaClO, which was again attributed to the higher DP of the final CNF. Moreover, Rodionova et al. (2012) reported that the final refining and homogenization process results in significantly higher defects and shorter lengths for hardwood nanofibers compared to softwood nanofibers; so, the correspondent tensile strengths of the softwood nanofiber films were significantly higher than the strengths of the hardwood nanofiber films.

\subsubsection{Copper determination}

As mentioned earlier, samples of TNFC from Red oak and Yellow poplar after the copper synthesis step (TNFC-Cu) were characterized by Energy-dispersive X-ray Analysis (EDX). The results show that both nanofibrillated hybrid materials (from red Oak and yellow Poplar) have similar copper weight percentage present after the synthesis process. This result can be attributed to the similar carboxylate content for both TNFC species, sites where copper is reduced from salts to the correspondent metallic state. This result is confirmed through the 
SEM analysis, presented in Figure 3.7, where the presence of copper is represented as crystals on the surface of TNFC-Cu from Red oak. Similar pictures were found for Yellow poplar. The presence of copper in the nanofibrillated hybrid material make possible to assume that the final hybrid material will possess antimicrobial properties as demonstrated in a previous research (Zhong et al. 2013).

\subsection{Conclusions}

Underutilized woody biomass composed by Red oak (Quercusrubra) and Yellow poplar (Liriodendron tulipifera) was used for producing TEMPO nanofibrillated cellulose (TNFC) using a chemi-mechanical treatment. The morphological analysis performed by TEM on TNFC indicated a final average fibril width between 3- $4 \mathrm{~nm}$ for both species. The carboxylate content after the TEMPO oxidation process resulted in approximately 0.8 $\mathrm{mmol} / \mathrm{g}$ for both species. No difference in the lignin composition trend was observed after the pulping and bleaching process for both woody species; hemicellulose content was higher for Red oak in all treated and untreated samples but showed no impact on the final morphology, carboxylate content or mechanical properties of the final TNFC. Model films prepared from Red oak and Yellow poplar evidenced similar thermo-mechanical properties, however, the strength of the two species seemed to be lower than the values published elsewhere. The low strength of films achieved in this research is probably due to the reduced DP after the mechanical treatment. Overall, both TNFC from Red oak and Yellow poplar, presented the same capability to support metallic copper to be used as hybrid materials in thermoplastic films for applications in the packaging and medical/pharmacy fields.

\section{ACKNOWLEDGEMENTS}

Funding for this work has been provided by NIFA McStennis WVA00098 "Efficient utilization of biomass for biopolymers in central Appalachia”, and USDA NIFA Grant No. 2013-34638-21481 "Development of novel hybrid cellulose nanocomposite film with potent biocide properties utilizing low quality Appalachian hardwoods". Special thanks to Changle Jiang, PhD student Forest Resources Science, for performing the copper determination analysis on TNFCs (EDX-SEM). 


\subsection{References}

Alemdar A, Sain M (2008) Isolation and characterization of nanofibers from agricultural residues-Wheat straw and soy hulls. Bioresource technology 99:1664-1671

Besbes I, Vilar MR, Boufi S (2011) Nanofibrillated cellulose from alfa, eucalyptus and pine fibres: preparation, characteristics and reinforcing potential. Carbohydrate Polymers 86:1198-1206

Brodin FW, Theliander H (2013) A comparison of softwood and birch kraft pulp fibers as raw materials for production of TEMPO-oxidized pulp, MFC and superabsorbent foam. Cellulose 20:2825-2838

Chen W, Yu H, Liu Y (2011) Preparation of millimeter-long cellulose I nanofibers with diameters of 30-80nm from bamboo fibers. Carbohydrate Polymers 86:453-461

Chirayil CJ, Joy J, Mathew L, Mozetic M, Koetz J, Thomas S (2014) Isolation and characterization of cellulose nanofibrils from Helicteres isora plant. Industrial Crops and Products 59:27-34

Deepa B. (2015) Utilization of various lignocellulosic biomass for the production of nanocellulose: a comparative study. Cellulose 22:1075-1090

Ferreira T, Rasband W (2012) ImageJ User Guide/IJ 1.46 r. Bioimage Informatics.

Fukuzumi H, Saito T, Isogai A (2013) Influence of TEMPO-oxidized cellulose nanofibril length on film properties. Carbohydrate Polymers 93:172-177

Henriksson M, Berglund LA, Isaksson P, Lindstrom T, Nishino T (2008) Cellulose nanopaper structures of high toughness. Biomacromolecules 9:1579-1585

Isogai A, Saito T, Fukuzumi H (2011) TEMPO-oxidized cellulose nanofibers. Nanoscale 3:71-85

Iwamoto S, Abe K, Yano H (2008) The effect of hemicelluloses on wood pulp nanofibrillation and nanofiber network characteristics. Biomacromolecules 9:10221026

Jacobson PM (2011) Properties of polyvinyl alcohol nanocomposites reinforced with cellulose nanocrystals of red oak residues. Master thesis at West Virginia University

Jiang C, Oporto GS, Zhong T, Jaczynski J (2016) TEMPO nanofibrillated cellulose as template for controlled release of antimicrobial copper from PVA films. Cellulose 23:713-722

Jin W, Singh K, Zondlo J (2013) Pyrolysis kinetics of physical components of wood and wood-polymers using isoconversion method. Agriculture 3:12-32

Jonoobi M, Niska KO, Harun J, Misra M (2009) Chemical composition, crystallinity, and thermal degradation of bleached and unbleached kenaf bast (Hibiscus cannabinus) pulp and nanofibers. BioResources 4:626-639

Kabir M, Wang H, Lau K, Cardona F (2013) Effects of chemical treatments on hemp fibre structure. Applied Surface Science 276:13-23 
Lani N, Ngadi N, Johari A, Jusoh M (2014) Isolation, characterization, and application of nanocellulose from oil palm empty fruit bunch fiber as nanocomposites. Journal of Nanomaterials 2014:13

Liu D, Liu Y, Sui G (2016) Synthesis and properties of sandwiched films of epoxy resin and graphene/cellulose nanowhiskers paper. Composites Part A: Applied Science and Manufacturing 84:87-95

Le Troedec $\mathrm{M}$ et al. (2008) Influence of various chemical treatments on the composition and structure of hemp fibres. Composites Part A: Applied Science and Manufacturing 39:514-522

Li J, Sevastyanova O, Gellerstedt G (2002) The relationship between kappa number and oxidizable structures in bleached kraft pulps. Journal of Pulp and Paper Science (JPPS) 28:262-266

Morais JPS, de Freitas Rosa M, Nascimento LD, do Nascimento DM, Cassales AR (2013) Extraction and characterization of nanocellulose structures from raw cotton linter. Carbohydrate Polymers 91:229-235

Maiti S, Jayaramudu J, Das K, Reddy SM, Sadiku R, Ray SS, Liu D (2013) Preparation and characterization of nano-cellulose with new shape from different precursor . Carbohydrate Polymers 98:562-567

Mark W. Davis (1998) A Rapid Modified Method for Compositional Carbohydrate Analysis of Lignocellulosics by High $\mathrm{pH}$ Anion-Exchange Chromatography withPulsed Amperometric Detection (HPAEC/PAD), Journal of Wood Chemistry and Technology,18:2, 235-252, DOI: 10.1080/02773819809349579.

Meng Q, Li H, Fu S, Lucia LA (2014) The non-trivial role of native xylans on the preparation of TEMPO-oxidized cellulose nanofibrils. Reactive and Functional Polymers 85:142-150

Nishiyama Y, Langan P, Chanzy H (2002) Crystal structure and hydrogen-bonding system in cellulose I $\beta$ from synchrotron X-ray and neutron fiber diffraction. Journal of the American Chemical Society 124:9074-9082

Pääkkönen T, Dimic-Misic K, Orelma H, Pönni R, Vuorinen T, Maloney T (2016) Effect of xylan in hardwood pulp on the reaction rate of TEMPO-mediated oxidation and the rheology of the final nanofibrillated cellulose gel. Cellulose 23:277-293

Qing Y, Sabo R, Zhu J, Agarwal U, Cai Z, Wu Y (2013) A comparative study of cellulose nanofibrils disintegrated via multiple processing approaches. Carbohydrate Polymers 97:226-234

Poddar PK, Gupta A, Jamari SS, Kim NS, Khan TA, Sharma S, Aziz MAA (2015) Synthesis of Nanocellulose from Rubberwood Fibers via Ultrasonication Combined with Enzymatic and Chemical Pretreatments. Asian Journal of Applied Sciences (ISSN: 2321-0893) 3 
Qing Y, Sabo R, Wu Y, Zhu J, Cai Z (2015) Self-assembled optically transparent cellulose nanofibril films: effect of nanofibril morphology and drying procedure. Cellulose 22:1091-1102

Retegi A. (2010) Bacterial cellulose films with controlled microstructure-mechanical property relationships. Cellulose 17:661-669

Rodionova G, Eriksen Ø, Gregersen Ø (2012) TEMPO-oxidized cellulose nanofiber films: effect of surface morphology on water resistance. Cellulose 19:1115-1123

Rodionova G, Saito T, Lenes M, Eriksen Ø, Gregersen Ø, Kuramae R, Isogai A (2013) TEMPO-mediated oxidation of Norway spruce and Eucalyptus pulps: preparation and characterization of nanofibers and nanofiber dispersions. Journal of Polymers and the Environment 21:207-214

Sain M, Panthapulakkal S (2006) Bioprocess preparation of wheat straw fibers and their characterization. Industrial Crops and Products 23:1-8

Saito T, Kimura S, Nishiyama Y, Isogai A (2007) Cellulose nanofibers prepared by TEMPO-mediated oxidation of native cellulose. Biomacromolecules 8:2485-2491

Saito T, Hirota M, Tamura N, Kimura S, Fukuzumi H, Heux L, Isogai A (2009) Individualization of nano-sized plant cellulose fibrils by direct surface carboxylation using TEMPO catalyst under neutral conditions. Biomacromolecules 10:1992-1996

Segal L, Creely J, Martin Jr A, Conrad C (1959) An empirical method for estimating the degree of crystallinity of native cellulose using the X-ray diffractometer Textile Research Journal 29:786-794

Siró I, Plackett D (2010) Microfibrillated cellulose and new nanocomposite materials: a review. Cellulose 17:459-494

Stelte W, Sanadi AR (2009) Preparation and characterization of cellulose nanofibers from two commercial hardwood and softwood pulps. Industrial \& Engineering Chemistry Research 48:11211-11219

Sun X, Xu F, Sun R, Fowler P, Baird M (2005) Characteristics of degraded cellulose obtained from steam-exploded wheat straw. Carbohydrate Research 340:97-106

Syverud K, Chinga-Carrasco G, Toledo J, Toledo PG (2011) A comparative study of Eucalyptus and Pinusradiata pulp fibres as raw materials for production of cellulose nanofibrils. Carbohydrate Polymers 84:1033-1038

Wu J, Sperow M, Wang J (2010) Economic feasibility of a woody biomass-based ethanol plant in central Appalachia. Journal of Agricultural and Resource Economics:522-

Wang Q, Zhu J, Gleisner R, Kuster T, Baxa U, McNeil S (2012) Morphological development of cellulose fibrils of a bleached eucalyptus pulp by mechanical fibrillation. Cellulose 19:1631-1643

Wicaksono R, Syamsu K, Yuliasih I, Nasir M, Street K (2013) Cellulose nanofibers from cassava bagasse: Characterization and application on tapioca-film. Chem Mater Res 3(13)79-87 
Wulandari W, Rochliadi A, Arcana I Nanocellulose prepared by acid hydrolysis of isolated cellulose from sugarcane bagasse. In: IOP Conference Series: Materials Science and Engineering, 2016. IOP Publishing, p 012045

Zhong T, Oporto GS, Jaczynski J, Tesfai A, Armstrong J (2013). Antimicrobial properties of the hybrid copper nanoparticles-carboxymethyl cellulose. Wood Fiber Sci. 45(2) 1-8

Zhong T, Oporto GS, Jaczynski J, Jiang C (2015a) Nanofibrillated cellulose and copper nanoparticles embedded in polyvinyl alcohol films for antimicrobial applications. BioMed research international Article ID 456834.

Zhong T, Oporto GS, Peng Y, Xie X, Gardner DJ (2015b) Drying cellulose-based materials containing copper nanoparticles. Cellulose 22:2665-2681

Zimmermann T, Bordeanu N, Strub E (2010) Properties of nanofibrillated cellulose from different raw materials and its reinforcement potential. Carbohydrate Polymers 79:1086-1093 
TABLES

Table 3.1 Conditions of pulping process, kappa number and screen yield after pulping.

\begin{tabular}{cc}
\hline Kraft pulping & \\
\hline Active alkali, \% Na2O & 24 \\
Maximum temperature & $165^{\circ} \mathrm{C}$ \\
Rise to maximum temperature & $1 \% \mathrm{~min}$ \\
Time at maximum temperature & $50 \mathrm{~min}$ \\
Liquor to solid ratio & 4 \\
Sulfidity & 25 \\
H-factor & 750 \\
Kappa number (after pulping) & RO: $13.25 ;$ YP: 20 \\
Screen yield pulping & RO: $51 \% ;$ YP: $52.4 \%$ \\
\hline
\end{tabular}

Table 3.2 Conditions of bleaching process.

\begin{tabular}{|c|c|c|c|c|c|}
\hline Stage & $\mathrm{D}$ & $\begin{array}{c}\text { Ep } \\
\text { (Alkaline } \\
\text { extraction } \\
\text { Stage 1) }\end{array}$ & $\begin{array}{c}\text { D } \\
\text { (Second } \\
\text { chlorine } \\
\text { dioxide) }\end{array}$ & Ep & $\mathrm{D}$ \\
\hline $\begin{array}{l}\text { Chemical } \\
\text { charge }\end{array}$ & $\begin{array}{l}\mathrm{CLO}_{2} \\
(1.7 \%) \\
\mathrm{H}_{2} \mathrm{SO}_{4} \\
(0.2 \%)\end{array}$ & $\begin{array}{c}\mathrm{NaOH}(2 \%) \\
\mathrm{H}_{2} \mathrm{O}_{2} \\
(0.38 \%)\end{array}$ & $\mathrm{ClO}_{2}(0.8 \%)$ & $\begin{array}{c}\mathrm{H}_{2} \mathrm{O}_{2} \\
(0.197 \%) \\
\mathrm{NaOH} \\
(1 \%)\end{array}$ & $\begin{array}{c}\mathrm{ClO}_{2} \\
(0.3 \%)\end{array}$ \\
\hline $\begin{array}{l}\text { Pulp } \\
\text { consistency }\end{array}$ & $10 \%$ & $10 \%$ & $10 \%$ & $10 \%$ & $10 \%$ \\
\hline End pH & 2.5 & 11 & 3.5 & 11 & \\
\hline Temperature & $70^{\circ} \mathrm{C}$ & $70^{\circ} \mathrm{C}$ & $70^{\circ} \mathrm{C}$ & $70{ }^{\circ} \mathrm{C}$ & $70^{\circ} \mathrm{C}$ \\
\hline Time & $45 \mathrm{~min}$ & $60 \mathrm{~min}$ & $80 \mathrm{~min}$ & $130 \mathrm{~min}$ & $60 \mathrm{~min}$ \\
\hline
\end{tabular}

D: Chlorine dioxide, E: peroxide reinforced alkaline extraction.

Table 3.3 Chemical characterization of untreated wood (Raw) and bleached pulps (B).

\begin{tabular}{ccccc}
\hline Sample & Cellulose\% & Hemicellulose\% & $\begin{array}{c}\text { Klason lignin } \\
(\%)\end{array}$ & $\begin{array}{c}\text { ASL* }^{*} \\
(\%)\end{array}$ \\
\hline RaWR.O & $53.8 \pm 0.6$ & $19.34 \pm 0.8$ & $22.5 \pm 0.2$ & 4.41 \\
RaWy.P & $56.8 \pm 0.7$ & $17.7 \pm 1.1$ & $22.3 \pm 0.5$ & 3.30 \\
BR.O & $78.4 \pm 1.6$ & $20.5 \pm 2.4$ & $1.1 \pm 0.7$ & 0.16 \\
B.P & $81.5 \pm 1.7$ & $17.6 \pm 2.1$ & $1.1 \pm 0.6$ & 0.15 \\
\hline
\end{tabular}

* ASL: Acid soluble lignin was measured just one time. 
Table 3.4 Viscosity and degree of polymerization of:Bleached samples and samples after TEMPO oxidation process (TOWP). Note: TOWP: TEMPO-oxidized wood pulp.

\begin{tabular}{ccc}
\hline Sample(s) & Viscosity(mPa s) & DP \\
\hline Bleached $_{(\text {R.O) }}$ & $19.4 \pm 0.4$ & $3152-3300$ \\
Bleached $_{(\text {Y.P) }}$ & $10.9 \pm 0.2$ & $1666-1736$ \\
TOWP(R.O) $_{\text {TOWP(Y.P) }}$ & $2.9 \pm 0.2$ & $361-421$ \\
TOW $_{\text {(Y) }}$ & $2.4 \pm 0.1$ & $302-332$ \\
\hline
\end{tabular}

Table 3.5 Carboxylic contents of Red oak and Yellow poplar species for bleached and Tempo oxidized samples (TOWP).

\begin{tabular}{cc}
\hline Samples & COOH $(\mathrm{mmol} / \mathrm{g})$ \\
\hline Bleached R.O & 0.05 \\
Bleached Y.P & 0.07 \\
TOWP.O & 0.82 \\
TOWPY.P & 0.80 \\
\hline
\end{tabular}

Table 3.6 Crystallinities of raw material, TEMPO-oxidized wood pulp (TOWP) and TEMPOoxidized cellulose nanofibers (TNFC).

\begin{tabular}{cc}
\hline Sample & Degree of Crystallinity (\%) \\
\hline RaW R.O & $37.0 \pm 3.26$ \\
RaW Y.P & $34.4 \pm 2.5$ \\
TOWPR.O & $61.7 \pm 1.66$ \\
TOWP Y.P & $59.85 \pm 2.2$ \\
TNFC R.O & $39.3 \pm 0.9$ \\
TNFCY.P & $39.4 \pm 0.5$ \\
\hline
\end{tabular}


Table 3.7 Onset degradation temperature (Tonset), maximum thermal degradation temperature (Tmax), maximum weight loss (WLmax) and CY (Char Yield) (\%): (Char residueafter $575{ }^{\circ} \mathrm{C}$ ).

\begin{tabular}{|c|c|c|c|c|c|c|}
\hline \multirow[t]{2}{*}{ Samples } & \multirow{2}{*}{$\begin{array}{c}\text { T onset }{ }^{\circ} \\
\text { C) }\end{array}$} & \multicolumn{2}{|c|}{ Stage I } & \multicolumn{2}{|c|}{ Stage ॥ } & \multirow{2}{*}{$\begin{array}{l}\text { CY } \\
(\%)\end{array}$} \\
\hline & & $\mathrm{T}_{\max }\left({ }^{\circ} \mathrm{C}\right)$ & $\begin{array}{c}\mathrm{WL}_{\max }(\% / \mathrm{o} \\
\mathrm{C})\end{array}$ & $\mathrm{T}_{\max }\left({ }^{\circ} \mathrm{C}\right)$ & $\begin{array}{c}\mathrm{WL}_{\max }(\% / \mathrm{o} \\
\mathrm{C})\end{array}$ & \\
\hline Raw R.o & 304.5 & 285 & 0.45 & 358 & 1.12 & 14.0 \\
\hline Raw Y.P & 312 & - & - & 366 & 1.17 & 13.4 \\
\hline $\begin{array}{c}\text { TNFC }_{R} . \\
0\end{array}$ & 224 & 234 & 0.58 & 288 & 0.68 & 20.0 \\
\hline $\begin{array}{c}\text { TNFCY. } \\
\text { P }\end{array}$ & 225 & 232 & 0.6 & 290 & 0.7 & 19.5 \\
\hline
\end{tabular}

Table 3.8 Mechanical Properties of Transparent Films of the Oxidized Cellulose Fibrils.

\begin{tabular}{|l|l|l|l|}
\hline Samples & $\begin{array}{l}\text { Tensile modulus ( } \\
\text { Gpa) }\end{array}$ & $\begin{array}{l}\text { Max tensile stress( } \\
\text { Mpa) }\end{array}$ & $\begin{array}{l}\text { Strain at } \\
\text { break (\%) }\end{array}$ \\
\hline TNFC $_{\text {RO }}$ & $12.35 \pm 1.02$ & $129.8 \pm 16.36$ & $7.75 \pm 1.4$ \\
\hline TNFC $_{Y P}$ & $13.6 \pm 2.09$ & $135.15 \pm 14.2$ & $6.41 \pm 2.04$ \\
\hline
\end{tabular}

Table 3.9 Copper determination using EDX of hybrid material TNFC-CuNPs based on TNFC from Red oak and Yellow poplar (three repetitions).

\begin{tabular}{ll}
\hline Samples & Cu (wt \%) \\
\hline TNFC $_{\text {R.O }}$ & $18.9 \pm 2.8$ \\
TNFC $_{Y . P}$ & $20.2 \pm 4.1$ \\
\hline
\end{tabular}


FIGURES

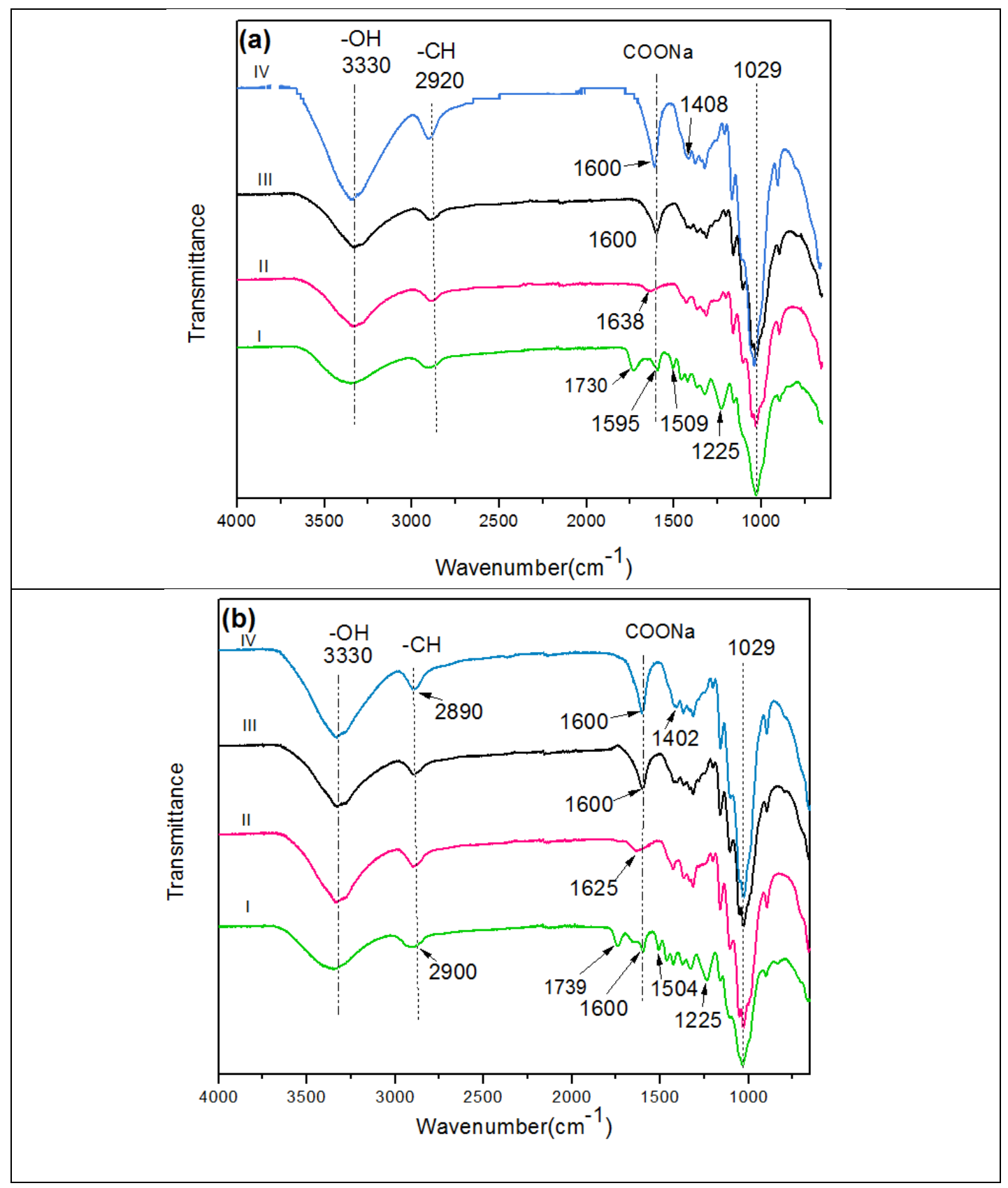

Figure 3.1 (a) FT-IR spectra of Red oak: (I)Raw material, (II)Bleached, (III)Tempo-oxidized Wood pulp (TOWP) and (IV)Tempo-oxidized cellulose nanofibers (TNFC). (b) FT-IR spectra of Yellow poplar: (I)Raw material, (II)Bleached, (III)Tempo-oxidized wood pulp (TOWP) and (IV)Tempooxidized cellulose nanofibers (TNFC). 

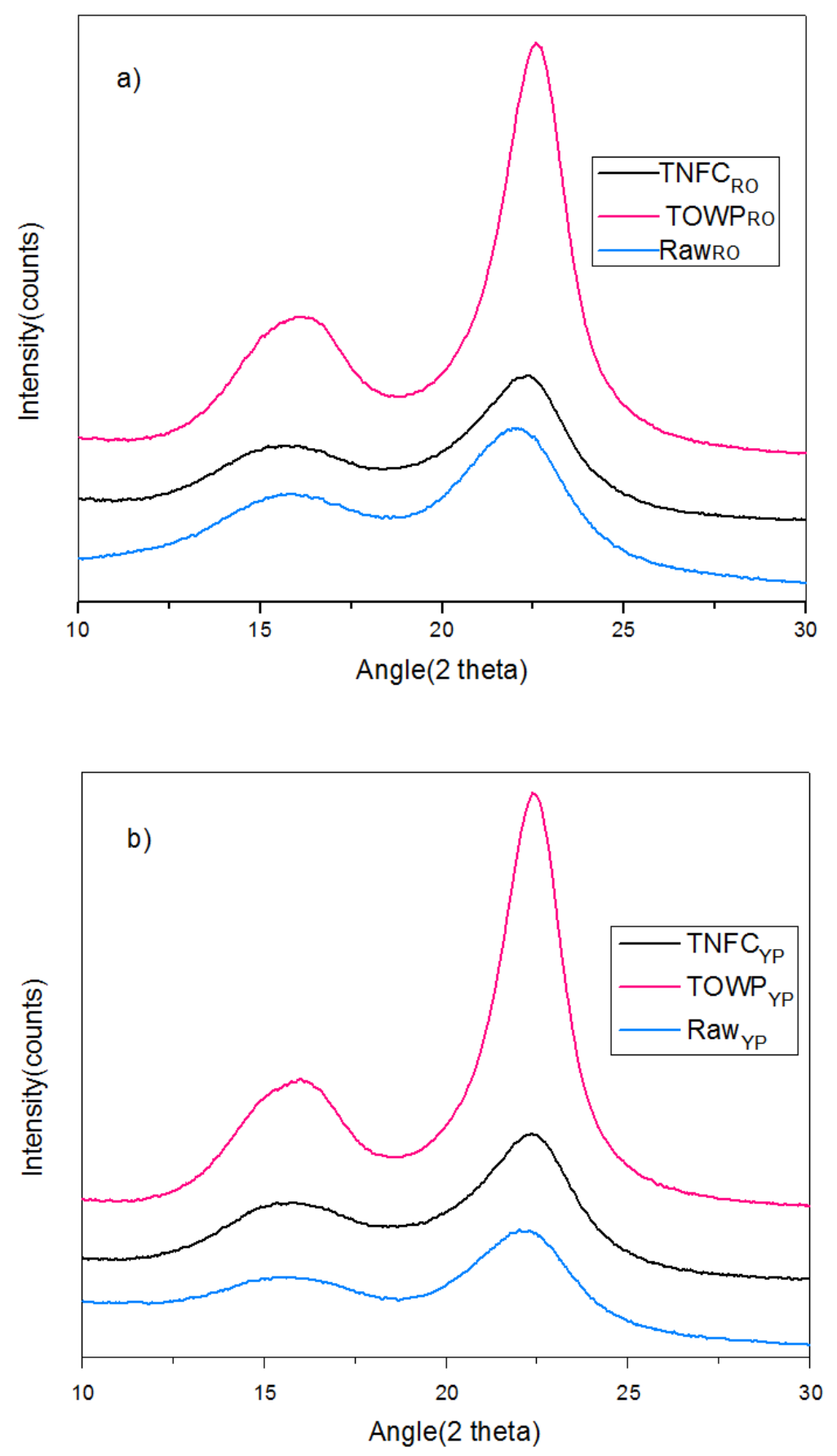

Figure 3.2 X-ray diffraction patterns of: a) Raw R.O,TOWPR.O, and TNFCR.O

; b) Raw Y.P, b: TOWPY.P, c: TNFCY.P 

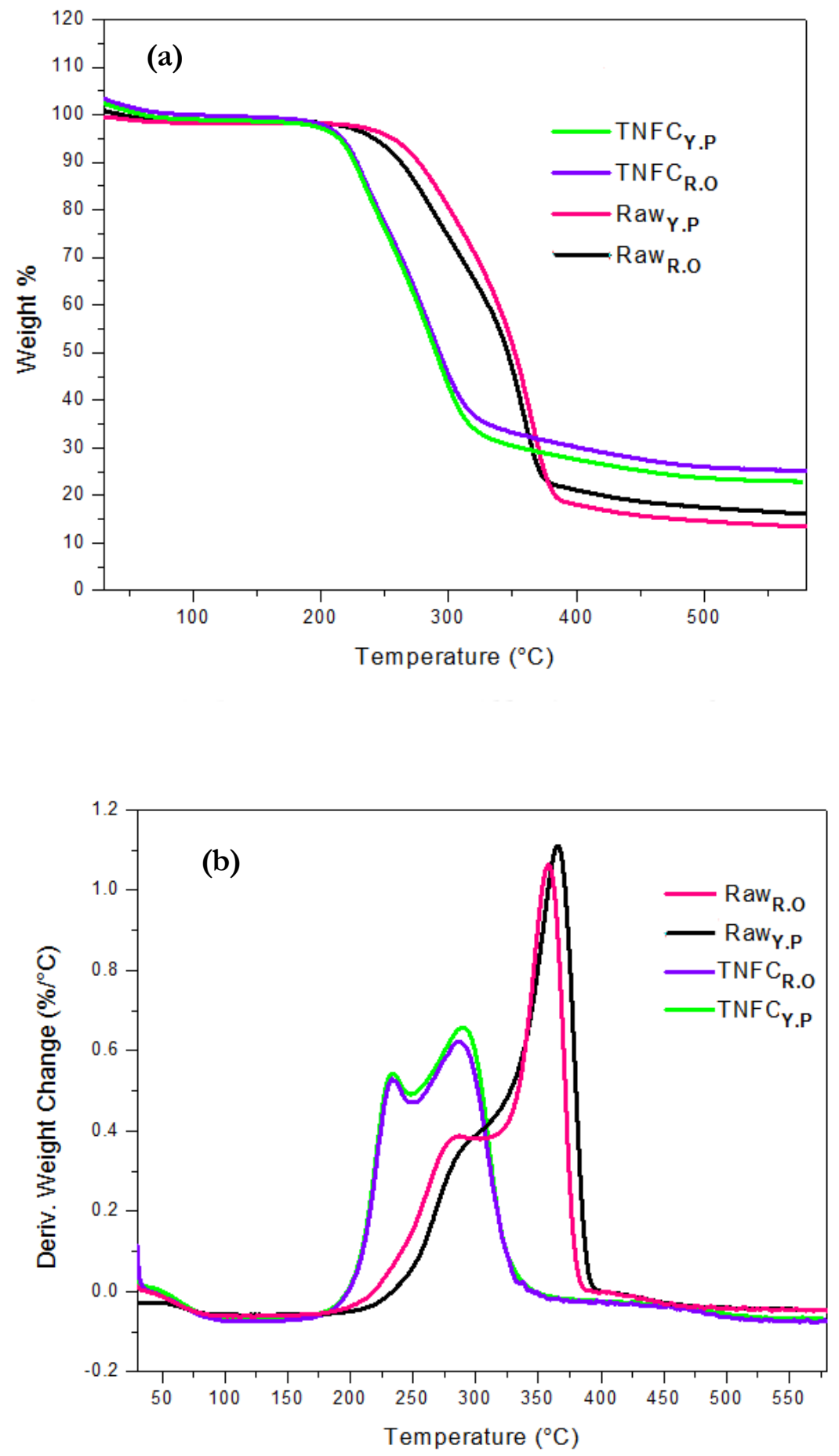

Figure 3.3 Thermogravimetric (TGA (a)) and the corresponding derivative thermogravimetric (DTG) (DTG (b)) curves of the raw material and TEMPO-oxidized cellulose nanofibers (TNFC) 


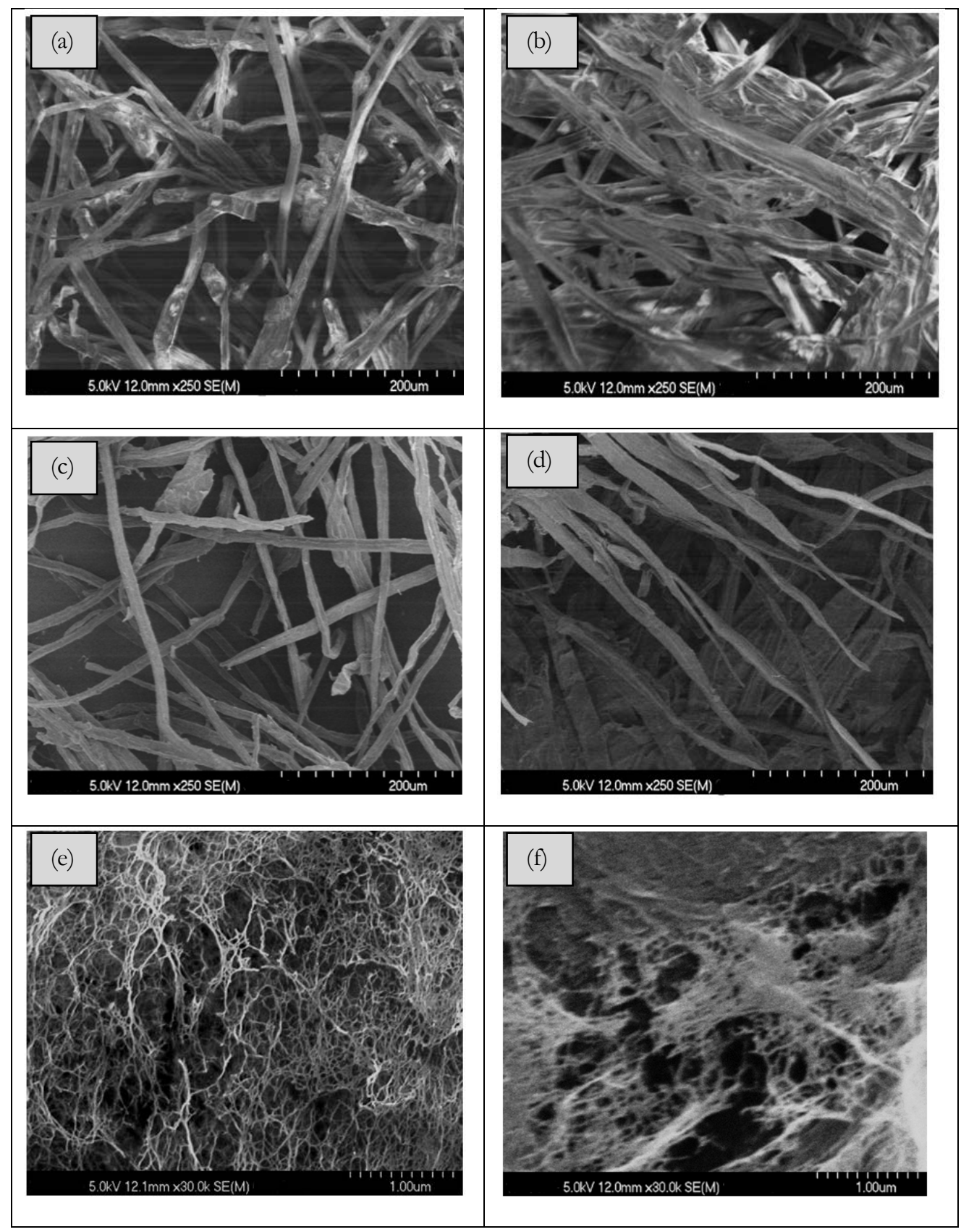

Figure 3.4 FE-SEM micrographs of the bleached R.O (a) and Y.P (b);TEMPO oxidized wood pulp (TOWP) of R.O (c) and Y.P (d); TEMPO nanofibrillated cellulose (TNFC) of R.O (e) and Y.P (f) samples after a solvent exchange(E/tert-B-FD treatment) followed by freeze drying. 


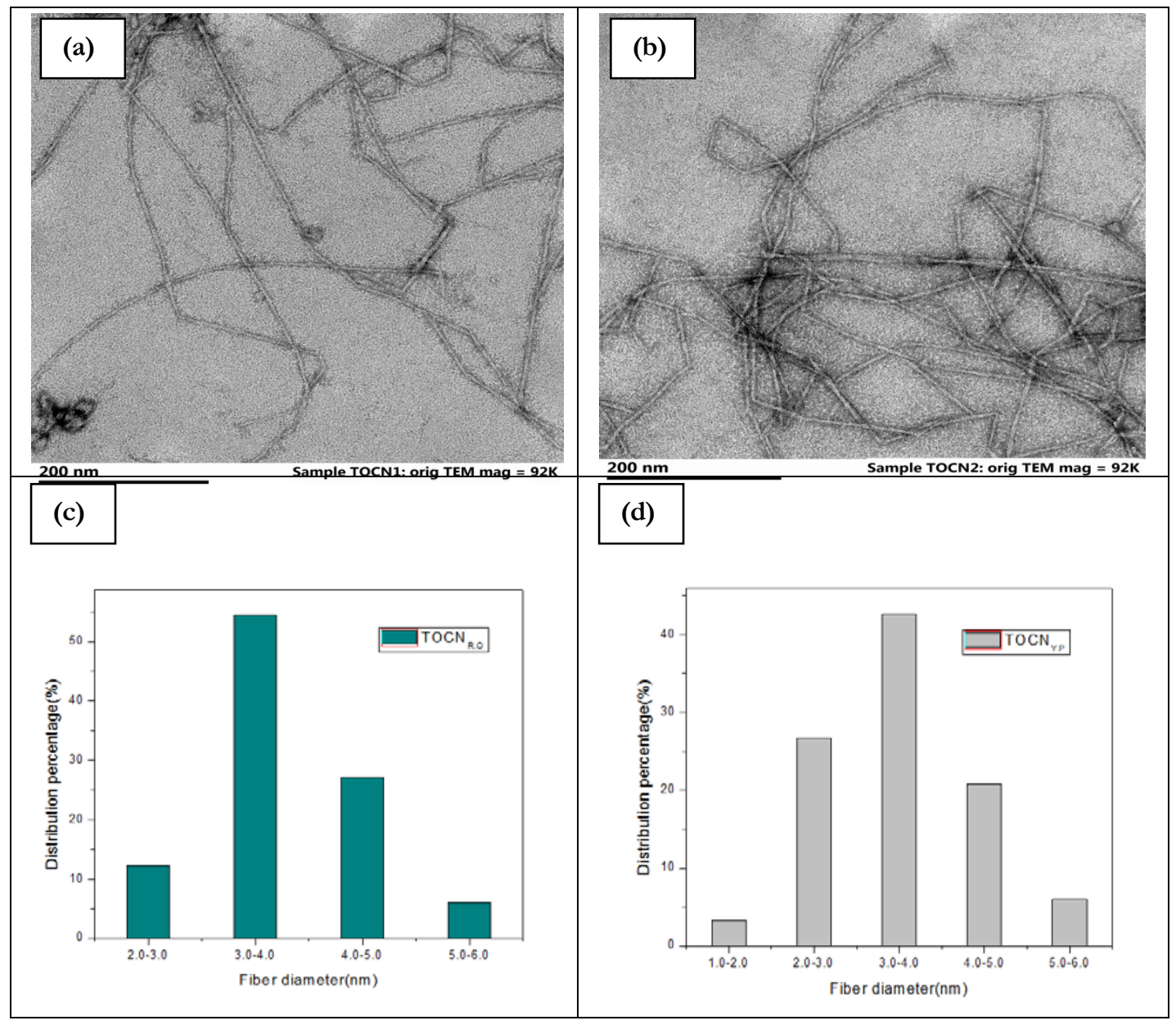

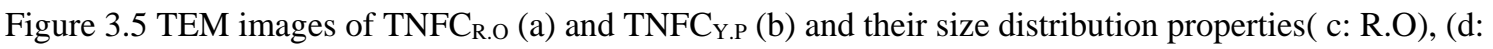
Y.P). 

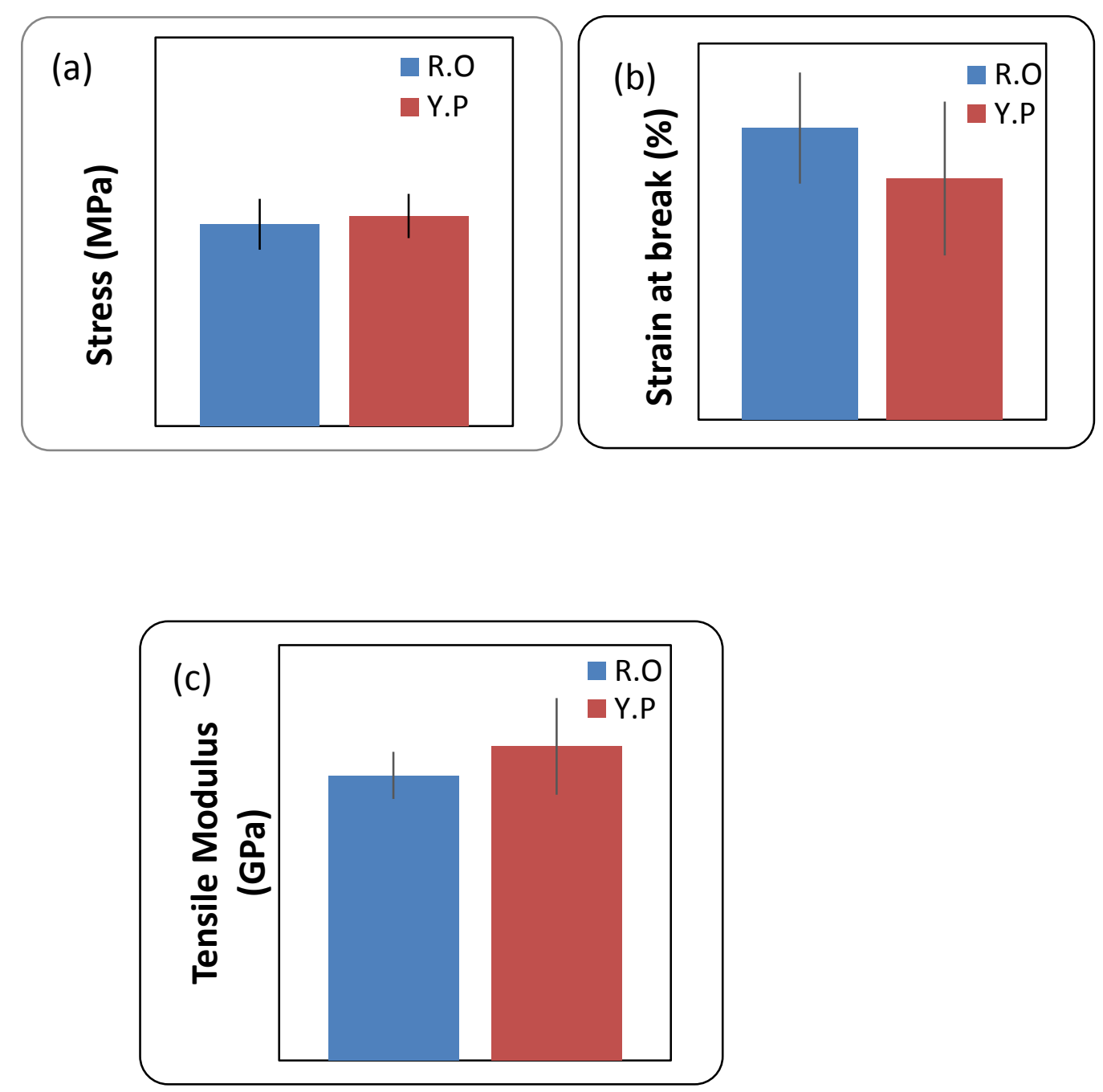

Figure 3.6 Main tensile properties of NFC films prepared from cellulose nanofibrils of R.O (R.O) and Y.P (Y.P). (a) Stress; (b) Strain at break; (c) Tensile modulus. 


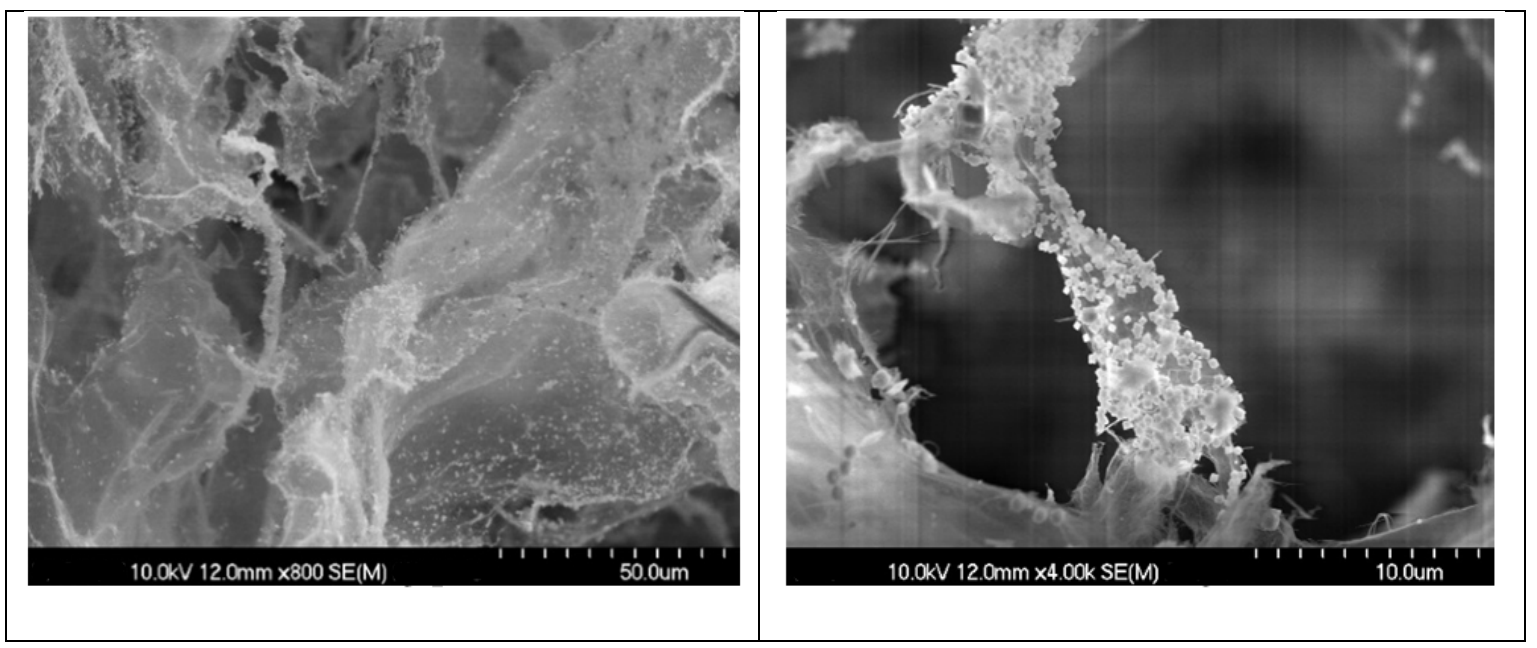

Figure 3.7 SEM images of dried TNFCRO after the treatment with copper sulfate and reducing agent sodium borohydride (TNFCR.O-Cu). 


\section{CHAPTER 4: MODIFIED NANOFIBRILLATED FROM THE APPALACHIAN HARDWOOD LOGGING RESIDUES}

\section{ABSTRACT}

The main goal of this research was to increase the carboxylic acid availability of TEMPO nanofibrillated cellulose and evaluate its effect on copper nanoparticles availability on the final nanocellulosic material. The raw materials used were from two dominant Appalachian hardwoods in West Virginia: Northern red oak (Quercus rubra) and Yellow poplar (Liriodendron tulipifera). Kraft pulps were obtained using the same procedure described in Chapter 3 and the bleaching procedure was modified to increase the carboxylic acid availability on the cellulosic material. This modification included the use of higher concentration of $\mathrm{NaClO}$. After the bleaching process, nanofibrillated cellulose was chemically modified using a TEMPO/NaBr/NaClO system and then metallic copper was incorporated on the cellulosic backbone through a chemical reduction. The carboxyl content of the oxidized cellulose was determined using titration method according to T237 om-88. The characterization of the untreated and treated samples was performed using X-ray diffraction (XRD), Fourier transform infrared spectroscopy (FTIR), transmission electron microscopy (TEM), scanning electron microscopy (SEM), SEM-EDX, and tensile strength determinations of the corresponding films. Red oak showed higher hemicellulose content than yellow poplar before the treatments, while after bleaching the hemicellulose content reached the same. Yellow poplar presented higher crystallinity, thermal stability and mechanical properties. The final Tempo-oxidized cellulose nanofibers (TNFC) presented similar morphology and diameter dimensions for both species (an average diameter of 3.2 $\mathrm{nm}$ in Red oak and Yellow poplar). The carboxyl content in both species was increased from $0.8 \mathrm{~m} \mathrm{~mol} / \mathrm{g}$ to 1.37 and $1.45 \mathrm{~m} \mathrm{~mol} / \mathrm{g}$ in Red oak and Yellow poplar. After increasing the carboxylic content on the cellulosic nanomaterial, there was no significant difference between the two species in terms of their copper availability. Both, Red oak and Yellow poplar copper content was approximately $20 \mathrm{wt} \%$ at the lower and higher carboxylic group concentration. 


\author{
Abreviations \\ CT Conductometric Titration \\ DP Degree of Polymerization \\ NFC Nanofibrillated cellulose \\ o.d.p Oven dried pulp \\ R.O Red oak \\ SEMscanning electron microscopy \\ TEMPO 2,2,6,6-tetramethylpiperidine-1-oxyl radical \\ TOWP TEMPO Oxidized Wood Pulp \\ TNFC TEMPO oxidized cellulose nanofibers \\ TEMtransmission electron microscopy \\ XRDX-ray diffraction \\ Y.P Yellow poplar
}




\subsection{Introduction}

As mentioned in Chapter 3, nanofibrillated cellulose (NFC) consists of long, flexible and entangled cellulose nanofibres, containing alternating crystalline and amorphous domains (Kalia et al. 2014). NFC key properties are their high specific area, ability to establish high hydrogen bonding, good mechanical strength, and high aspect ratio (Missoum et al. 2013). Nanofibrillated cellulose (NFC) can be disintegrated from cellulose pulp through a waterbased disintegration process, which yields fibrils with a diameter in the range 5-100 nm. Such fibrils provide excellent forming ability and large surface area for an effective functionalization (Sehaqui et al. 2015).

Nanofibrillated cellulose (NFC) is a promising material to be used in several fields that include, among others, the packaging area (as oxygen barrier coating), biomedical (Kalia et al. 2014), food additive (Rodionova et al. 2013), heavy metal ion adsorbent (Isobe et al. 2013), regenerative medicine (De Carvalho et al. 2016) etc.

The surface properties of NFC can be changed depending on the specific application from hydrophilic to hydrophobic or from anionic to cationic. In particular, carboxylic cellulosic nanofibers with uniform diameters from 3 to $5 \mathrm{~nm}$ can be obtained through a 2,2,6,6tetramethyl-1-piperidinyloxy free radical (TEMPO) mediated oxidation pretreatment of the cellulose pulp before disintegration, a selective treatment which converts the primary alcohol of the glucose ring to negatively charged carboxylate entity (Sehaqui et al. 2015). TEMPO is a highly stable nitroxyl radical which is used extensively in the selective oxidation of primary alcohols to corresponding aldehydes and carboxylic acids (Kalia et al. 2014).

The NFCs obtained by TEMPO-mediated oxidation show properties of high crystallinity, mostly uniform widths (3-4 nm), and large aspect ratios (>100). Moreover, the presence of negatively charged sodium carboxylate groups on the surface of the CNFs makes it possible to completely disperse them at the individual nanofibril level in water by electrostatic repulsion and/or osmotic effects (Carlsson et al. 2014). Figure 4.1 shows the chemical mechanism of cellulose surface modification during TEMPO-mediated oxidation, where also the intermediate oxidation product, aldehyde groups, is shown. The oxidation is selective to 
the primary $\mathrm{O}(6)$ hydroxyls on the surface of cellulose nanofibrils, and, if conducted under controlled conditions, does not result in dissolution of cellulose or morphological and structural changes (Carlsson et al. 2014; Saito and Isogai 2004).
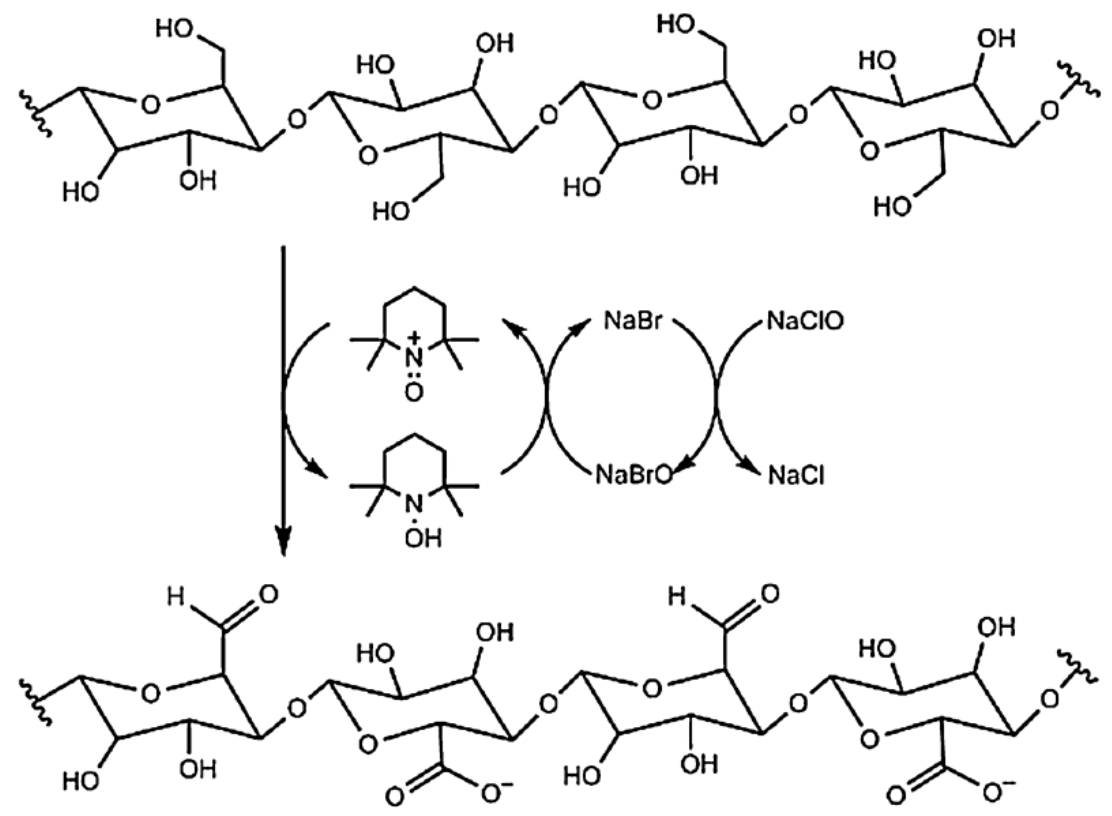

Figure 4.1 Reaction scheme for surface modification of cellulose with TEMPO-mediated oxidation (Carlsson et al. 2014).

The carboxylate content on the surface of NFC can be tailored by various amounts of $\mathrm{NaClO}$ as the primary oxidant (Liu et al. 2016b).

In this context, Saito et al.2007 examined associations between the amount of $\mathrm{NaClO}$ added as the co-oxidant and either the carboxylate content of the TEMPO-oxidized cellulose or the oxidation time for never-dried and once-dried celluloses used as the starting materials. Here, 1 mol of the C6 primary hydroxyl group was converted to 1 mol of C6 carboxylate group via C6 aldehyde by consuming $2 \mathrm{~mol}$ of $\mathrm{NaClO}$. The amount of carboxylate groups formed from the primary hydroxyl groups of cellulose increased with the amount of $\mathrm{NaClO}$ added.

Dong et al. (2013) introduced carboxylated groups onto the surface of NFCs and used them to prepare hydrogels by triggering strong ionic interactions between the carboxylate groups and cationic metal salts. The cellulose nanofibril-based hydrogels may have a variety of 
biomedical and other applications, taking advantage of their biocompatibility, high porosity, high surface area, and durability in water and organic solvents (Dong et al. 2013b).

Negatively charged carboxyl groups provided by TEMPO can also be considered as binding sites for various macromolecules such as peptides and enzymes (Paukkonen et al. 2017), polyelectrolytes and nanoparticles (Sehaqui et al. 2014), adsorbed surfactants on cellulose surface and may facilitate fiber homogenization process (Rodionova et al. 2013). The very large surface area and negative charge of NFCs also suggests that large amounts of drugs might be bound to the surface of this material with the potential for high payloads and optimal control of dosing (Paukkonen et al. 2017).

In Chapter 3 we demonstrate a feasible procedure to produce TEMPO nanofibrillated cellulose using highly available hardwoods logging residues from West Virginia. In the present work we focused our research in a methodology to increase the amount of carboxylic groups on the nanocellulosic material and evaluating the effect of copper availability after this chemical modification. Oxidized nanofibers were prepared from logging residues of Red oak and Yellow poplar using the TEMPO-mediated oxidation approach. By applying more aggressive bleaching process and more concentration of oxidant agent in TEMPO-mediated oxidation process, the carboxylate groups introduced on the nanofibers surface has been doubled. Our hypothesis is that the increase of carboxylate groups (negative sites) will favour the electrostatic attraction of positive copper ions $\left(\mathrm{Cu}^{2+}\right)$.

\subsection{Materials and methods}

\subsubsection{Fiber samples}

Northern red Oak (Quercusrubra) and yellow Poplar (Liriodendron tulipifera) collected in 2014 at WVU Research Forest, Preston County, West Virginia were used in the form of logging residue. The samples were chipped into short fibers and screened. The fraction retained between $1 / 2$ inch opening and 3/8 inch was used for pulping. 


\section{Pretreatments}

\subsubsection{Pulping procedure}

The process for TNFC production including pulping procedure was performed at Forest Product Laboratory, Madison, Wisconsin. The fraction passing through a screen with 1/2inch openings and retained by a 3/8-inch screens was used. Pulping was carried out in an M/ K System 6-L laboratory digester using liquors prepared from reagent grade sodium hydroxide and sodium sulphide. The ratio of cooking liquid to fibres was 4:1. The maximum cooking temperature was set to $165^{\circ} \mathrm{C}$, and this temperature was reached after $50 \mathrm{~min}$. All pulping chemical charges, including $\mathrm{EA}(\mathrm{NaOH}+1 / 2 \mathrm{Na} 2 \mathrm{~S})$, are expressed in terms of $\mathrm{Na} 2 \mathrm{O}$. Sulfidity is expressed as a percentage of active alkali $(\mathrm{NaOH}+\mathrm{Na} 2 \mathrm{~S})$, and the expected H-factor would be 750 .

Table 4.1 lists the conditions of the pulping process. After $50 \mathrm{~min}$ at the maximum temperature, the temperature was reduced rapidly. The cooked samples were fibrillated, and then washed using vacuum filtration.

After pulping, the material was disintegrated by vigorous agitation and screened on a Valley flat screen (0.008 inch slot) then washed and collected in an additional 200 mesh screen. The screened samples were used for next step (bleaching). The resulted oven dried pulps were characterized in terms of their lignin content via Kappa number determination. The Kappa numbers of the two species were determined by measuring the consumption of potassium permanganate according to T $236 \mathrm{~cm}-85$ standard method. The percentage of lignin for the resultant kraft pulps was determined using the following equation:

Lignin level $(\%)=$ Kappa number $\times 0.15 \quad$ Eq.1

The kappa number (KN) of the kraft pulp was 17.5 and 35.5 giving the lignin percentage of 2.62 and 5.32 for R.O and Y.P after kraft pulping, respectively. 


\subsubsection{Bleaching}

Pulps were bleached by $\mathrm{D}_{0} \mathrm{EpD}_{1} \mathrm{EpD}_{2}$ bleaching sequences. (Where $\mathrm{D}$ explains Chlorine dioxide (ClO2), E refers to peroxide reinforced alkaline extraction). In the first stage $\left(\mathrm{D}_{0}\right)$ $\mathrm{ClO}_{2}$ charge was 1.7 and 2.8\% for R.O and Y.P, respectively which has been set based on their Kappa number content. Bleaching experiment was performed in sealed plastic jars. The pulp was thoroughly washed between stages. After all stages the fibers were washed and oven dried.

Table 4.2 shows the conditions of the bleaching processes.

\subsubsection{TEMPO-Mediated Oxidation}

Bleached samples were used for the TEMPO- mediated oxidation process. Around $30 \mathrm{~g}$ oven dried of bleached samples were added into 3 (lit) water that contains TEMPO (0.016 g per gr pulp) and sodium bromide (0.1 g/gr pulp). The TEMPO-mediated oxidation reaction started by adding $\mathrm{NaClO}$ solution (6.0 mmolNaClO per gram of pulp), and continued at room temperature by stirring at $500 \mathrm{rpm}$. The $\mathrm{pH}$ was maintained at 10 by adding $0.5 \mathrm{M} \mathrm{NaOH}$ using a pH stat until no $\mathrm{NaOH}$ consumption was observed. Extra amount of $\mathrm{NaClO}$ was added into each sample as long as no changes in $\mathrm{pH}$ happened, meaning that no consumption and no hydroxyl group remained. The TEMPO-oxidized cellulose was thoroughly washed with water by filtration and stored at $4{ }^{\circ} \mathrm{C}$ before homogenization(Saito et al. 2007).

Samples of each species were air dried, then used for characterization such as DP (degree of polymerization), and CT (Conductometric Titration). The rest of the TEMPO- Oxidized samples were diluted to have $1 \%$ solid content before homogenization.

\subsubsection{Production of nanofibrils}

Mechanical fibrillation was performed by passing the treated material (around 1wt\% suspension of TEMPO oxidized cellulose) through an M-110EH-30 Microfluidizer (Microfluidics, Newton, MA). Three passes through a 100 micron diamond orifice at 138 MPa feed pressure was done on each oxidized pulp sample.

Characterizations of the fibers and nanofiber suspensions 


\subsubsection{Fiber chemical composition}

Samples of kraft and bleached pulp were first dried, and then milled by 20 mesh willey mill (model No.2, Arthur H.Thomasco). These samples together with untreated samples were used for chemical composition analyses. The procedure used for cellulose determination was given by Davis (Davis 1998). Anion exchange chromatography Dionex instrument (a ICS3000 system) were applied for sugar contents of hydrolysates. Lignin, were determined based on the amounts of sulfuric acid-insoluble Klason lignin, in accordance with the standard of the Technical Association of Pulp and Paper Industry (TAPPI) TAPPI standards-T222 om 88.

\subsubsection{Intrinsic viscosity and degree of polymerization}

Viscosity of resulting nanofibrillated cellulose was measured according to TAPPI Standard Method T230 om-99. Oven dried cellulosic solids of $0.1 \mathrm{~g}$ was first dispersed with $10 \mathrm{~mL}$ distilled water, and then added $10 \mathrm{~mL}$ of $1 \mathrm{M}$ cupriethylenediamine solution $(0.5 \%$ solid concentration). The viscosity of the resultant solution was determined with a capillary viscometer. The degree of polymerization (DP) was estimated by the following equation:

$\mathrm{DP}=120 \times[\eta]^{1.11}$

Where $\eta$ is the measured viscosity (Alexander et al. 1957); Results are presented in Table 4.4 .

\subsubsection{Carboxyl content of the pulp}

Determination of carboxyl content of the samples before and after the TEMPO-mediated oxidation process was performed according to T 237 om-88, modified as indicated below to account for the high acid content of the fiber with some reagent concentration modifications. The pulps were converted to the acid form by soaking them in $0.01 \mathrm{M} \mathrm{HCl}$ and then washing with deionized water. This acidified pulp was reacted with a standardized $0.1 \mathrm{M} \mathrm{NaHCO} 3$ with $0.25 \mathrm{M} \mathrm{NaCl}$ solution. The amount of residual $\mathrm{NaHCO}_{3}$ was determined by titration with 0.1 M HCl, to a methyl red endpoint. The carboxyl content was calculated in milliequivalents 
(meq) per $100 \mathrm{~g}$ of oven-dry pulp and then converted to $\mathrm{mmol} / \mathrm{g}$ and the results are presented in Table 4.5.

\subsubsection{Fourier transform infra-red spectroscopy (FTIR)}

Fourier transform infrared spectra were recorded using a Perkin-Elmer Spectrum 2000 FTIR spectrometer. The samples were analyzed from 600 to $4,000 \mathrm{~cm}^{-1}$. The spectrum obtained from a dry sample using 16 scans at a $4 \mathrm{~cm}^{-1}$ resolution and a $1 \mathrm{~cm}^{-1}$ interval at room temperature.

\subsubsection{X-ray diffraction (XRD) characterization}

X-ray diffraction (XRD) analysis was performed on the raw material, TEMPO-oxidized pulps, and the final TEMPO nanofibrillated cellulose (TNFC) samples. The analysis was performed on a X-Ray diffractometer (PANalyticalX'Pert Pro XRD) in the range of $10^{\circ}$ to $40^{\circ} 2 \theta$. Cu-ka1 $8047.2 \mathrm{eV}$ source and a maximum X-Ray power of $45 \mathrm{kV}$ and $40 \mathrm{~mA}$ were used. The crystallinity index(CI) after the different treatments was determined using the peak height method and calculated by the Segal empirical method (Eq.3)(Segal et al. 1959) after subtraction of the background signal:

$$
\mathrm{CI}(\%)=\frac{\mathrm{I} 200-\mathrm{I} \mathrm{am}}{\mathrm{I} 200} \times 100 \quad \text { Eq.3 }
$$

Where $I_{200}$ is the peak intensity corresponding to crystalline cellulose $\mathrm{I}$ at $2 \theta=22.6^{\circ}$ and $\mathrm{I}_{\mathrm{am}}$ the intensity minimum between the 200 and 110 peaks $\left(2 \theta=18^{\circ}\right)$

\subsubsection{Thermogravimetric analysis (TGA)}

Thermogravimetric analysis was carried out with 4-6 mg sample size to determine the thermal decomposition of the fibers after each treatment. The thermal stability data were obtained on a TA Q50 thermogravimetric analyzer (Delaware, USA), under linear temperature conditions. The temperature was set from $25^{\circ} \mathrm{C}$ to $600{ }^{\circ} \mathrm{C}$ at a heating rate of 10 ${ }^{\circ} \mathrm{C} / \mathrm{min}$ under a nitrogen atmosphere with nitrogen at a flow rate of $20 \mathrm{ml} / \mathrm{min}$.

\subsubsection{Morphological characterization}

Scanning electron microscopy (SEM) was used to study the effects of different treatments on the fiber morphology. Bleached, TOWP (TEMPO-Oxidized wood pulp)samples of each 
species were air-dried while TNFC (TEMPO-Oxidized cellulose nanofibers after homogenization) samples were freeze-dried after a preliminary solvent exchange process using ethanol and tert-butanol (Zhong et al. 2015).

TNFC samples were solvent exchanged from water to the less-polar solvent ethanol and tertbutanol in order to reduce the amount of hydrogen bonding between the fibers and the likelihood of aggregate formation during drying. TNFC Samples were collected after each processing stage and were separated from water by centrifugation at $3500 \mathrm{~g}$ for $10 \mathrm{~min}$. These steps were repeated three times for ethanol and three times for tert-butanol to replace most of the water with alcohol. The obtained suspensions of cellulose in alcohol were frozen in liquid nitrogen and dried in high vacuum using a freeze-dryer.The morphological analyses of the samples were characterized using a Hitachi S-4700 FE-SEM to study the effect of the treatments on the fiber morphology; all the samples were coated with gold-palladium using a sputtering process to provide adequate conductivity.

For TEM analysis, NFC samples of R.O and Y.P were diluted to 0.1\%, diluted NFC suspension was deposited to the glow-discharged copper grid with formvar and carbon film (400 mesh). The droplet was maintained on the grid for 2 min, and then rinsed thoroughly using a $2 \%$ aqueous uranyl acetate stain followed by blotting dry. Samples wereimaged using Philips CM-100 TEM (Philips/FEI Corporation, Eindhoven, Holland) operated at 100 kv, spot 3,200-lm condenser aperture, and 70-lm objective aperture. The images were captured using a SIA L3C 4-2M pixel CCD camera (Scientific Instruments and Application, Duluth, GA, USA) with $92 \mathrm{k}$ magnification. The diameter of different CNFs was measured and calculated from TEM images using the ImageJ Manipulation Program(Ferreira and Rasband 2012).

\subsubsection{Preparation of bybrid TNFC and copper nanoparticles}

The hybrid TNFC-copper nanoparticles (TNFC-CuNPs) were prepared according to (Jiang et al. 2016; Zhong et al. 2015a). Upon dispersing TNFC-CuNPs into deionized water, it was dried using a preliminary solvent exchange process with ethanol/tert-butanol and then freeze dried as described in (Zhong et al. 2015b). 


\subsubsection{Copper determination on TNFC from Red oak and Yellow poplar}

Copper determination of TNFC-CuNPs was conducted using Energy Dispersive X-ray (EDX) analysis and the results are presented in Table 4.9. The morphological analysis of the TNFC-CuNPs from red oak and yellow poplar were performed using a Hitachi S-4700 FESEM; the samples were previously coated with gold-palladium using a sputtering process to provide adequate conductivity. The morphology of the samples was analyzed by SEM and is presented in Figure 4.4 (e) and (f).

\subsection{Results and discussion}

\subsubsection{Chemical analysis}

The modification of plant fibers may involve the swelling of the crystalline region and the removal of the hydrophilic hydroxyl groups. This can be commonly achieved by a gradual decrease of amorphous components like lignin and hemicellulose from a raw fiber to a bleached sample. A five-stage bleaching sequence (DEpDEpD) was performed in order to remove the residual lignin. Multi-stage bleaching sequences are customarily divided into delignification and bleaching segments. The D-stages were meant to oxidize the lignin, making fragments smaller, more soluble and oxidize the chromophores, in turn making the pulp brighter/whiter. The alkali extraction was meant to further enhance lignin solubility, as well as to activate the remaining lignin for a more effective bleaching in the next D-stage. The dissociation of hydrogen peroxide in alkaline media generates the peroxide anion (HHO-), which is mainly responsible for the reduction in the concentration of chromospheres, leading to brighter bleached fibers (Bufalino et al. 2015). The successful pretreatments of the raw materials are attested by the changes in carbohydrate contents (Table 4.3). As expected, the results indicate a notorious difference before and after kraft pulping and bleaching. Based on the chemical composition analysis, a majority of the lignin was removed from the bleached pulps of the two species.

The cellulose content increased from 54\% and 57\% in R.O and Y.P raw materials to 78.7\% and $80.7 \%$ after bleaching, whereas the lignin contents decreased from $22.3 \%$ and $21.9 \%$ in 
R.O and Y.P to $0.7 \%$ and 1.1\%followed by bleaching step, respectively. In all treated and untreated samples, cellulose content was higher in Y.P.

Lignin is a complex organic compound with an alkali soluble character; hence the percentage of lignin decreases from a raw fiber to bleached fiber due to alkali extraction in the bleaching step.

The kappa number (KN) of the kraft pulp was 17.62 and 35.5 resulting to 2.65 and 5.32 lignin percentage for R.O and Y.P, respectively. The kappa number includes acid soluble lignin as well as other groups that react with the permanganate, such as hydroxyl methylfurfural. The kappa number varies according to the wood species and delignification procedure during pulping. Other compounds than lignin oxidized by $\mathrm{KMnO} 4$ like hexenuronic acid will increase its consumption, and therefore the Kappa number (Li et al. 2002).

It should be noticed that the hemicellulose content was not changed after kraft and bleaching process in both samples but when compared to each other, The RO had more hemicellulose than YP.

Taking into account that the purification was performed to remove non-cellulose components, mainly lignin, the purification process has reached its goal due to the low content of lignin and high content of cellulose in the purified samples.

\begin{tabular}{|l|l|l|l|l|l|l|l|l|l|}
\hline Sample & KlasonLig. & $\begin{array}{l}\text { Lig. } \\
\text { Ash }\end{array}$ & Arabanan & Galactan & Glucan & Xylan & Mannan & $\begin{array}{l}\text { Tot. } \\
\text { Carb. }\end{array}$ & $\begin{array}{l}\text { Tot. } \\
\text { Yield }\end{array}$ \\
\hline $\begin{array}{l}\text { RAW } \\
\text { RO }\end{array}$ & $22.3 \%$ & $0.1 \%$ & $0.55 \%$ & $1.66 \%$ & $37.63 \%$ & $17.15 \%$ & $2.21 \%$ & $59.21 \%$ & $81.6 \%$ \\
\hline $\begin{array}{l}\text { RAW } \\
\text { YP }\end{array}$ & $21.9 \%$ & - & $0.60 \%$ & $0.60 \%$ & $43.01 \%$ & $15.53 \%$ & $2.39 \%$ & $62.13 \%$ & $83.9 \%$ \\
\hline RO-K & $1.5 \%$ & $0.1 \%$ & & & & & & & \\
\hline YP-K & $4.7 \%$ & $0.3 \%$ & ND & $0.56 \%$ & $58.05 \%$ & $15.22 \%$ & $1.13 \%$ & $74.96 \%$ & $79.9 \%$ \\
\hline RO-B & $0.7 \%$ & $0.1 \%$ & ND & ND & $55.95 \%$ & $19.50 \%$ & $1.03 \%$ & $76.48 \%$ & $77.3 \%$ \\
\hline YP-B & $1.1 \%$ & $0.1 \%$ & ND & ND & $67.12 \%$ & $16.59 \%$ & $1.51 \%$ & $85.22 \%$ & $86.4 \%$ \\
\hline
\end{tabular}

The RO bleached samples has a larger fraction of xylose and less glucose compared to the YP. This trend has been seen before in our previous work. Alkaline treatment not surprisingly is able to significantly remove xylans from pulps. This indicated that a portion of the xylans 
was alkali-stable likely due to strong hydrogen bonding between xylans and cellulose fibrils, a result in accordance to what was observed by Pejicet al.

It has been reported that part of the xylans can precipitate or resorb onto the fiber surface during kraft pulping. Furthermore, a small amount of stable hemicellulose cannot be removed by alkali, the so-called intra-microfibrillar xylan that most likely is located inside the microfibrils and between elemental fibrils (Syverud et al. 2011) .

High xylan content is together with easier fibrillation (Meng et al. 2014; Syverud et al. 2011). Obviously, xylans are the clearly most predominant hemicellulose in R.O. All other sugars such as galactan, and arabinan were well below the detection limits after bleaching.

During conventional kraft cooking, part of the hemicellulose dissolves in the liquor. The hemicellulose dissolves steadily throughout cooking, and at the same time the dissolved hemicellulose undergoes degradation. For hardwood, theconcentration of xylan in the cooking liquor reaches a maximum (about $8 \%$ of the wood weight) and then slowly decreases as the cooking is continued. For softwood, the maximum concentration is reached at a later stage of cooking and the concentration of dissolved xylan is lower compared with hardwood. An earlier study on radial distribution of hemicellulose through the cell wall of unbleached and bleached spruce kraft pulps concluded that the major portion of kraft hemicellulose, especially xylan, were located in the outermost layers of the fiber (Shin and Stromberg 2007).

\subsubsection{Degree of polymerization}

Table 4.4 gives the estimated DP of the bleached and TEMPO-oxidized would pulp (TOWP) samples of the two applied species. The results indicate that the DP is consequently higher for R.O fibers in bleached samples and the corresponding Tempo-oxidized cellulose, compared to the Y.P samples. The DP of the bleached R.O and Y.P pulps dropped within 1.5 h of oxidation, from 3207 to 380 and from 1718 to 302, respectively. The TEMPO-mediated oxidation and especially the increase of the $\mathrm{NaClO}$ content inevitably lead to proportional de-polymerization of the starting material in both samples of R.O and Y.P, but showed more decrease in Y.P samples.Change of degree of oxidation has been found to show an obvious effect on the size of NFC. DPv values and average lengths of TEMPO-oxidized cellulose nanofibrils were decreased with increasing addition of $\mathrm{NaClO}$ in the TEMPO-mediated 
oxidation stage (Kalia et al. 2014) which is in coincidence with our previous and current results.

\subsubsection{Carboxyl content}

Table 4.5 shows that the TOWP contained higher carboxylate content ,the increase of $\mathrm{NaClO}$ concentration over the pretreatment range of $6 \mathrm{M}$ caused an increase in carboxylate contents in TEMPO oxidized fibers from 0.80 to 1.37 and $1.45 \mathrm{mmol} / \mathrm{g}$ o.d.pfor R.O and Y.P, respectively.

As these species showed very little difference between their hemicellulose content ( 20.53\% and $18.1 \%$ in RO and YP bleached pulp, respectively), which in turn may explain one reason for the similarity between the two pulps with respect to the amount of charged groups (Syverud et al. 2011).

However, the slight difference in carboxylate content can be attributed to the Xylan level after bleaching in two species. The amounts of Xylan after bleaching were 19.5 and 16.7\% in RO and YP, respectively. This might be one reason for having higher carboxyl content in YP compared to RO. As reported by Meng et al. xylans without the C6 primary hydroxyls are not oxidized during TEMPO oxidation(Meng et al. 2014).

\subsubsection{FTIR spectroscopy analysis}

The analysis of functional groups through FTIR is presented in Figure 4.2 a) for R.O. and 1b) for Y.P., where (a) corresponds to the initial woody raw material; (b) are the bleached samples; (c) corresponds to the TEMPO nanofibrillated material (TNFC).

More intensive peaks have been observed in raw samples in following ranges:

1730( $\mathrm{C}=\mathrm{O}$ stretching of the acetyl and uronic ester groups of hemicellulose or the ester linkage of carboxylic groups of ferulic and p-coumaric acids of lignin and/or hemicellulose (Alemdar and Sain 2008; Bufalino et al. 2015; Sun et al. 2005) ), 1593 (stretching of aromatic rings of lignin (Bufalino et al. 2015),1503 (the $-\mathrm{C}=\mathrm{C}$ - stretch of the aromatic rings of lignin (Nacos et al. 2006; Sain and Panthapulakkal 2006; Sun et al. 2005), this peak is the most characteristic bond for lignin; 1456 (attributed to lignin (Nacos et al. 2006), 1230(C-O stretching of the aryl group in lignin (Le Troedec et al. 2008). 
The lower amount or disappearance of this peak from the spectra of the bleached pulp fibers as well as from those of the nanofibers could be explained by the removal of lignin due to chemical treatments.

More intensive peaks in 3335 and 2899,1029, 898 after treatments and new peaks in 1601, $1406 \mathrm{~cm}^{-1} 1$ have been observed in TNFC samples of both RO and YPwhichare described as below:

3335: hydrogen bonded hydroxyl $(\mathrm{OH})$ groups of cellulose and absorbedwater, 2899: aliphatic saturated C-H stretching vibration (Poddar et al. 2015; Wicaksono et al. 2013; Wulandari et al. 2016). These peaks showed to be more intensive after bleaching and Tempooxidation in each species compared to before treatments.

Two strong absorption bands at around 1638-1600 and 1412-1402 $\mathrm{cm}^{-1}$, derived from the carbonyl groups $-\mathrm{C}=\mathrm{O}$ present in response to the TEMPO-mediated oxidation of cellulose nanofibers, indicated that hydroxyl groups at the C6 position of cellulose molecules were converted to sodium carboxylate.These two ranges were higher after TEMPO mediated oxidation in the order of TNFC=TOWP $>$ bleached $>$ raw material samples in R.O and Y.P separately, while it showed no special difference between the two species(Nacos et al. 2006).A strong carboxylate contribution is observed at about $1615-1611 \mathrm{~cm}^{-1}$, as expected (Nacos et al. 2006).

The peak at $1029 \mathrm{~cm}-1$ for both hardwood species seems to be much higher for TNFC in following the trend TNFC > bleached > raw material in R.O and Y.Pmeaning more carbohydrate content after treatment.This peak represents the stretching bond of C-O and O$\mathrm{H}$ and bending vibration of the $\mathrm{C}-\mathrm{O}-\mathrm{C}$ pyranose ring in cellulose molecules (Lani et al. 2014; Liu et al. 2016a).

A very characteristic peak at $898 \mathrm{~cm}^{-1}$ indicates the dominance of b-glycosidic links in the of the $\beta$-glycosidic link in cellulose (Jiang and Hsieh 2013).

Overall, comparing Figure 4.2 (a) and (b), YP bleach represented 1730-40(lignin), while RO bleach represented more 3335 bond showing higher content of cellulose, and the typical absorption band that appears in R.O and Y.P were in accordance with other experiments such as chemical composition content. 


\subsubsection{Crystallinity change}

Crystalline structure of cellulose in the cellulose fibrils is not uniform. The crystalline regions are extensively distributed throughout the fiber and are connected through less ordered (amorphous) inter-linking regions. Crystallinity of the cellulose sample can vary, depending on themethod of production as well as characterization techniques (Rambabu et al. 2016).

Figure 4.3 shows the diffractograms of the bleached, TEMPO-Oxidized fibers and the fibers obtained after different 3 numbers of passes through the microfluidizer.

The crystallinityindex (CI) of the bleached samples was52- $53 \%$ for both samples, respectively. After oxidation, the apparent crystallinity of samples increased to about $62 \%$ in R.O and Y.P, respectively owing to the removal of lignin. The crystallinity after TEMPO oxidation washigher than the bleached samples due to the oxidation which might be due to the removal of hemicellulose.The little increasing in the crystallinity after TEMPO oxidization due to partial loss of the disordered regions during the washing process , was also observed by Saito \&Isogai (Cao et al. 2012).

However, the crystalline degree went through significant reduction upon the homogenization process to a degree of 44 and $48 \%$ in TNFC species of R.O and Y.P, respectively. Mechanical fibrillation appears to break apart the crystalline and amorphous region of cellulose, resulting in decreasing crystallinity for TNFC samples. This breakage of cellulose crystals is believed to contribute to the separation of microfibrils and its bundles. The TEMPO-oxidized cellulose was subjected to three passes through the microfluidizerand previous reports showed upon extensive mechanical fibrilation cellulose crystallinity decreases due to the destruction of cellulose crystals because of high shear forces on the crystal surface, thus reducing the crystal size. Reduction of the cellulose crystal size leads to lower crystal volume and hence to a decrease in crystallinity (Rambabu et al. 2016).

\subsubsection{Thermal gravimetric analysis}

In order to have an estimation of thermal degradation characteristics of the untreated and chemo-mechanical treated R.O and Y.P, we performed Thermogravimetric (TGA) and 
derivative thermogravimetric (DTG) analyses. Figure 4.6 presents details of the major decomposition temperatures and weight losses percentage.

Figure 4.6(a), shows the TGA curve represented by weight (\%). The thermal degradation of untreated raw samples begins at temperatures 304.5 and $312{ }^{\circ} \mathrm{C}$ ( $\mathrm{T}_{\text {onset }}$ ), whereas in TEMPO oxidized NFCs the Tonset began at $216^{\circ} \mathrm{C}$ for R.O and 222 for Y.P. (Figure 5.(b)) shows the DTG curve represented by deriv. weight $\left(\% /{ }^{\circ} \mathrm{C}\right)$. Based on that, both TEMPO-oxidized and untreated samples showed a prominent pyrolysis process, with one step degradation for TNFC samples. The maximum weight loss was observed in the temperature range from 233$285^{\circ} \mathrm{C}$ and $358-366^{\circ} \mathrm{C}$, respectively.

The untreated R.O showed a small broadening or shoulder at $285^{\circ} \mathrm{C}$ on the left side of the main peak ( $366^{\circ} \mathrm{C}$ ), as shown in the DTG curves in Figure 4.6(b) which may have been due to the decomposition of hemicellulose. Hemicellulose has lower thermal stability than lignin and cellulose (Chirayil et al. 2014)so would be degraded before cellulose and lignin. the DTG curve showed a less sharp peak on the left side of the main peak, In the case of the untreated Y.P, which may be explained by the less content of hemicellulose. This probably resulted in better thermal properties for the untreated Y.P than R.O (304.5 versus 312 in Table 4.7 for raw R.O and raw Y.P, respectively) as the decomposition of hemicellulose beginning at much lower temperatures, compared to cellulose (Kabir et al. 2013).

Pyrolysis of cellulose is the last major decomposition peak observed at the temperature range between $300-400^{\circ} \mathrm{C}$. This peak was higher in Y.P compared to R.O in raw fibres andTNFC samples and might be explained by the fact that Y.P showed a higher cellulose content than R.O by carbohydrate measurement (Table 4.3); the trend is also in accordance with crystallinity where TNFC Y.P showed higher crystallinity resulting to have higher thermal degradation.

As expected, the degradation temperature of TEMPO oxidized NFCs for both R.O and Y.P, were less than those of untreated samples, because most of the lignin and hemicellulose were removed during the previous pulping and bleaching processes and because the carboxylic 
acid groups initiate thermal decomposition at lower temperatures (Deepa et al. 2015; Fukuzumi et al. 2008). These results are consistent with the results obtained from FTIR and XRD measurements.

Compared to preious work, the Tonset for TNFC samples of two species is lower this time, that might be because of higher amount of carboxyl group in the present work (Hassanzadeh et al. 2017)( 1.37 and $1.45 \mathrm{mmol} / \mathrm{g}$ Compared to 0.8 in previous paper).

Also, as presented in Table 4.7, the amounts of char residues (CY\%) from TNFC samples were higher than that of raw samples. The large amount of char residue form TEMPO oxidized NFCs may be due to their nano-sized fibres and higher number of carboxylate groups on their surface (Soni and Mahmoud 2015). In our first attempt (Hassanzadeh et al. 2017)the CY\% of the two samples were lower than the current same samples probably due to having higher degree of carboxyl content.

\subsubsection{Morphology of the treated and untreated samples}

SEM images of bleached samples of R.O and Y.P, and TEMPO oxidized fibers (TOWP) of R.O, and Y.P, are presented in Figure 4.4 (a) and (b)(c) and (d), respectively. Before the analysis the samples were dried in a conventional laboratory oven at $103^{\circ} \mathrm{C} \pm 2^{\circ} \mathrm{C}$ overnight. According to the SEM images of bleached samples (3(a), (b)), Y.P fibers seem to be more agglomerated than the correspondent R.O fibers which is in accordance with chemical analysis as klason lignin is almost twice after bleaching in $\mathrm{YP}(1.1 \%)$ compared to R.O $(0.7 \%)$. Based on Figure 4.4 (c) and (d) the TEMPO oxidation process has not affected the fibers diameter; however, qualitatively it is possible to observe less aggregation of fibers after the TEMPO oxidation process compared to the bleaching.

Figure 4.4 (e) and (f) are SEM images of TNFC forms of R.O and Y.P loaded with copper, respectively. Before the analysis the samples were dried using a solvent exchange process previous a freeze drying stage (Zhong et al. 2015b).

TEM images of TEMPO nanofibrillated cellulose (TNFC) from R.O and Y.P respectively are presented in Figure 4.5 (a) and (b). The diameter of these TNFCs was measured from 
TEM images using the imagej Manipulation Program (Ferreira and Rasband 2012). The average diameter for TNFC from R.O resulted of $3.2 \pm 0.66 \mathrm{~nm}$ and of $3.3 \pm 0.74 \mathrm{~nm}$ for TNFC from Y.P. that is less than the previous work (Hassanzadeh et al. 2017)which can be because of a different mechanical disintegration procedure which we applied (three passes through microfluidizer compared to two passes in previous attempt).

The distribution of diameter percentages of nanofibrils are presented in Figure 4.5 (c) and (d) for TNFC R.O and TNFC Y.P, respectively. Both samples had the most distribution percentage of 51-52\% for fibrils in 3-4 nm diameters, while 41 and 27\% of nanofibrils were in 2-3 nm range for R.O and Y.P, respectively.

\subsubsection{Mechanical test results}

The tensile modulus (modulus of elasticity or MOE) of the various NFC films was calculated based on the initial linear region of the stress-strain curve and is shown in Table 4.8. Y.P showed more MOE (22.2 \pm 1.8 GPa versus $14.9 \pm 4.66$ in R.O). Previous studies by other groups showed that tensile modulus strongly depends on the crystallinity of nanofibrils.The results of MOE in both species was in accordance with XRD analysis by which TNFC in Y.P samples showed more crystallinity after homogenization (Table 4.6).

The strain at break for the various NFC films was $2.45 \pm 0.8$ and $2.17 \pm 0.4$ for RO and YP, Respectively. The maximum tensile stress (strength) of films prepared from different NFCs is shown in Table 4.8. It can be seen the TEMPO films from Y.P had higher mechanical strength with 178.2 \pm 7.9 MPa compared to R.O (116.7 $\pm 25.5 \mathrm{MPa})$.

Rodionova et al. 2012 reported that the final refining and homogenization process results in significantly higher defects and shorter lengths for hardwood nanofibers compared to softwood nanofibers; so the correspondent tensile strengths of the softwood nanofiber filmswere significantly higher than the strengths of the hardwood nanofiber films.

\subsubsection{Copper determination}

Energy-dispersive X-ray Analysis (EDX) was used to estimate the concentration of copper on TNFC surfaces. The copper percentage was 23 and 17\% for R.O and Y.P, respectively (Table 4.9). Compared to the previous work with carboxylic content of $0.8 \mathrm{mmol} / \mathrm{l}$ there has not been an increase in copper percentage. The reason might be the same amount of copper 
which has been used for both sample compared to the first trial. In this context, Liu at al.(Liu et al. 2016b), found that TNFC loaded with 0.6 and TNFC $1.5 \mathrm{mmol} / \mathrm{g}$ carboxylic acid, have almost the same copper adsorption amount when the copper concentration remains at low levels (initial $\mathrm{Cu}(\mathrm{II}) \leq 10 \mathrm{mg} / \mathrm{g}$ ), which is likely due to the relative abundance of the carboxylate groups functioning as metal-binding groups for biosorption. Copper adsorption increased when the initial copper concentration was more than $20 \mathrm{mg} / \mathrm{g}$. In our study, the copper concentration was the same for 0.8 and $1.4 \mathrm{mmol} / \mathrm{g}$ carboxylate content $(\leq 10 \mathrm{mg} / \mathrm{g})$ which might explain while the copper percentage did not change significantly with increasing carboxylate content. This study may continue to see the effect of the initial concentration of copper on surface absorption.

\subsection{Conclusions}

The carboxyl content for Red oak and Yellow poplar increased from $0.8 \mathrm{mmol} / \mathrm{g}$ to 1.37 and $1.45 \mathrm{~m} \mathrm{~mol} / \mathrm{g}$.

XRD results revealed that chemical treatment also improved crystallinity of fibers to almost $62 \%$ after TEMPO but reduced to 44 and $48 \%$ in RO and YP cellulose nanofibres by homogenization, respectively. Higher crystallinity value of the fiber is expected to provide better reinforcement and therefore better properties of the composite films. In this context, the maximum tensile strength and Young's modulus of the nanocellulose film were $178 \mathrm{MPa}$ and 22.2 GPa for YP.

The average diameter for TNFC from R.O resulted of $3.2 \pm 0.66 \mathrm{~nm}$ and of $3.3 \pm 0.74 \mathrm{~nm}$ for TNFC from Y.P. that is less than the previous work (Chapter 3). This might be produced because of a different mechanical disintegration procedure. In this work three passes through the microfluidizer were used instead of two passes in previous work.

Compared to previous work, the $\mathrm{T}_{\text {onset }}$ for TNFC samples of two species is lower this time that might be because of higher amount of carboxyl group in the present work (1.37 and 1.45 $\mathrm{mmol} / \mathrm{g}$ Compared to 0.8 in previous paper). 
In Chapter 3 the $\mathrm{CY} \%$ of the two samples was lower than the current same samples probably due to having higher degree of carboxyl content.

As these species showed very little difference between their hemicellulose content (20.53\% and $18.1 \%$ in RO and YP bleached pulp, respectively), which in turn may explain one reason for the similarity between the two pulps with respect to the amount of charged groups. The amounts of Xylan after bleaching were 19.5 and $16.7 \%$ in RO and YP, respectively. This might be one reason for having higher carboxyl content in YP compared to RO as reported by Meng et al.

$\mathrm{DPv}$ values and average lengths of TEMPO-oxidized cellulose nanofibrils were decreased with increasing addition of $\mathrm{NaClO}$ in the TEMPO-mediated oxidation stage (Kalia et al. 2014) which is in coincidence with our previous and current results.

The cellulose content increased from 54\% and 57\% in R.O and Y.P raw materials to 78.7\% and $80.7 \%$ after bleaching, whereas the lignin contents decreased from $22.3 \%$ and $21.9 \%$ in R.O and Y.P to $0.7 \%$ and $1.1 \%$ followed by bleaching step, respectively. In all treated and untreated samples, cellulose content was higher in Y.P.

The RO bleached samples has a larger fraction of xylose and less glucose compared to the YP. This trend has been seen before in our previous work (chapter 3). Alkaline treatment not surprisingly is able to significantly remove xylans from pulps. This indicated that a portion of the xylans was alkali-stable likely due to strong hydrogen bonding between xylans and cellulose fibrils, a result in accordance to what was observed by Pejicet al.

For copper determination on the final TEMPO nanofibrillated cellulose, and considering the EDX standard deviation, there was no significant difference between the two species in terms of copper availability. However more variability was found in red oak samples. Also, compared to the previous work, the copper was not much affected by the carboxylic group 
availability. Both, red oak and yellow poplar copper content was approximately $20 \mathrm{wt} \%$ at the lower and higher carboxylic group content.

\section{Acknowledgements}

Funding for this work has been provided by NIFA McStennis WVA00098 "Efficient utilization of biomass for biopolymers in central Appalachia”, and USDA NIFA Grant No. 2013-34638-21481 "Development of novel hybrid cellulose nanocomposite film with potent biocide properties utilizing low quality Appalachian hardwoods”.

Table 4.1 Conditions of pulping process.

\begin{tabular}{cc}
\hline Kraft pulping & \\
\hline Active alkali, \% Na2O & 24 \\
Maximum temperature & $165^{\circ} \mathrm{C}$ \\
Rise to maximum temperature & $1 \% \mathrm{~min}$ \\
Time at maximum temperature & $50 \mathrm{~min}$ \\
Liquor to solid ratio & 4 \\
Sulfidity & 25 \\
H-factor & 750 \\
\hline
\end{tabular}

Table 4.2 Conditions of bleaching process.

\begin{tabular}{|c|c|c|c|c|c|}
\hline Stage & D0 & $\begin{array}{c}\text { Ep(Alkaline } \\
\text { Extraction } \\
\text { Stage 1) }\end{array}$ & $\begin{array}{c}\text { D1 (Second } \\
\text { Chlorine } \\
\text { Dioxide) }\end{array}$ & Ep & D2 \\
\hline $\begin{array}{l}\text { Chemical } \\
\text { charge }\end{array}$ & $\begin{array}{l}\mathrm{CLO}_{2} \\
2.8 \%) \\
\mathrm{H}_{2} \mathrm{SO}_{4} \\
(0.2 \%)\end{array}$ & $\begin{array}{c}\mathrm{NaOH}(2 \%) \\
\mathrm{H}_{2} \mathrm{O}_{2} \\
(0.38 \%)\end{array}$ & $\mathrm{ClO}_{2}(1 \%)$ & $\begin{array}{c}\mathrm{H}_{2} \mathrm{O}_{2}(0.197 \%) \\
\mathrm{NaOH}(1 \%)\end{array}$ & $\mathrm{ClO}_{2}(0.6 \%)$ \\
\hline $\begin{array}{l}\text { Pulp } \\
\text { consistency }\end{array}$ & $10 \%$ & $10 \%$ & $10 \%$ & $10 \%$ & $10 \%$ \\
\hline End pH & 2.5 & 11 & 3.5 & 11 & \\
\hline Temperature & $70^{\circ} \mathrm{C}$ & $70^{\circ} \mathrm{C}$ & $70^{\circ} \mathrm{C}$ & $70^{\circ} \mathrm{C}$ & $70^{\circ} \mathrm{C}$ \\
\hline Time & $45 \mathrm{~min}$ & $60 \mathrm{~min}$ & $80 \mathrm{~min}$ & $130 \mathrm{~min}$ & $60 \mathrm{~min}$ \\
\hline
\end{tabular}


Table 4.3 Chemical characterization of Kraft pulps (K), Bleached pulps (B), and untreated samples (Ref) of Red Oak: R.O , Yellow poplar: Y.P.

\begin{tabular}{|c|c|c|c|}
\hline Sample & $\begin{array}{c}\text { Klason } \\
\text { lignin (\%) }\end{array}$ & Hemicellulose (\%) & Cellulose (\%) \\
\hline RefR.O $_{\text {R. }}$ & 22.3 & 18.75 & 54 \\
\hline Ref..P & 21.9 & 16.9 & 57 \\
\hline Kraftro & 1.5 & 1.83 & 96.5 \\
\hline KraftyP & 4.7 & 1.69 & 93.3 \\
\hline Bleached RO & 0.7 & 1.03 & 98.2 \\
\hline BleachedYP & 1.1 & 1.51 & 97.3 \\
\hline
\end{tabular}

Table 4.4 Viscosity and degree of polymerization of: TOWPR.o, TOWPY.P: Samples after TEMPO oxidation, and Bleached samples. Note: TOWP: TEMPO-oxidized wood pulp.

\begin{tabular}{ccc}
\hline Sample(s) & Viscosity(mPa s) & DP \\
\hline Bleached(R.O) $_{\text {Bleached(Y.P) }}$ & $19.3 \pm 0.3$ & 3207.4 \\
TOWP(R.O) $_{\text {TOWP(Y.P) }}$ & $11 \pm 0.1$ & 1718.4 \\
TOW $_{(}$ & $2.83 \pm 0.15$ & 380.8 \\
\hline
\end{tabular}

Table 4.5 Carboxylic contents of R.Oak and Y.Poplar species in bleached and Tempo oxidized samples.

\begin{tabular}{cc}
\hline Samples & COOH $(\mathrm{mmol} / \mathrm{g})$ \\
\hline Bleached R.O & 0.05 \\
Bleached Y.P & 0.07 \\
TOC R.O & 1.37 \\
TOC $_{\text {Y.P }}$ & 1.45 \\
\hline
\end{tabular}

Table 4.6 Cristallinities of cellulose fibers at different stages; TNFC: TEMPO nanofibrillated cellulose, TOWP: Tempo-oxidized wood pulp, Raw: Original samples of Red Oak and Yellow Poplar.

\begin{tabular}{cc}
\hline Sample & Degree of Crystallinity (\%) \\
\hline Bleach R.O & $53 \pm 1.4$ \\
Bleach Y.P & $52.5 \pm 3.5$ \\
TOWP R.O & $61.5 \pm 2.12$ \\
TOWP Y.P & $62.5 \pm 3.5$ \\
TNFC R.O & $44.23 \pm 3.7$ \\
TNFC Y.P & $48.8 \pm 1.03$ \\
\hline
\end{tabular}


Table 4.7 Onset degradation temperature ( $\left.T_{\text {onset}}\right)$, maximum thermal degradation temperature $\left(\mathrm{T}_{\max }\right)$, maximum weight loss (WLmax) and CY (Char Yield) (\%): (Char residueafter $575^{\circ} \mathrm{C}$ )

\begin{tabular}{|c|c|c|c|c|c|c|}
\hline \multirow[t]{2}{*}{ Samples } & \multirow[t]{2}{*}{$\mathbf{T}_{\text {onset }}\left({ }^{\circ} \mathrm{C}\right)$} & \multicolumn{2}{|c|}{ Stage I } & \multicolumn{2}{|c|}{ Stage II } & \multirow[t]{2}{*}{ CY (\%) } \\
\hline & & $\mathrm{T}_{\max }\left({ }^{\circ} \mathrm{C}\right)$ & $\mathrm{WL}_{\max }\left(\%^{p} \mathrm{C}\right)$ & $\mathrm{T}_{\max }\left({ }^{\circ} \mathrm{C}\right)$ & $\mathrm{WL}_{\max }(\% / \mathrm{C})$ & \\
\hline Raw R.O & 304.5 & 285 & 0.45 & 358 & 1.12 & 14.0 \\
\hline Raw Y.P & 312 & - & - & 366 & 1.17 & 13.4 \\
\hline TNFC $_{\text {R.O }}$ & 216.3 & 234 & 0.66 & - & - & 27.0 \\
\hline TNFCy.P & 222 & 237 & 0.58 & - & - & 21.5 \\
\hline
\end{tabular}

Table 4.8 Mechanical Properties of Transparent Films of the Oxidized Cellulose Fibrils

\begin{tabular}{|l|l|l|l|}
\hline Samples & Tensile modulus ( Gpa) & Max tensile stress( Mpa) & $\begin{array}{l}\text { Strain at break } \\
(\mathbf{\%})\end{array}$ \\
\hline TNFC $_{\mathbf{R O}}$ & $14.9 \pm 4.66$ & $116.7 \pm 25.5$ & $2.45 \pm 0.8$ \\
\hline TNFC & $22.2 \pm 1.8$ & $178.2 \pm 7.92$ & $2.17 \pm 0.4$ \\
\hline
\end{tabular}

Table 4.9 Copper determination using EDX of hybrid material TNFC-CuNPs based on TNFC from Red oak and Yellow poplar (three repetitions).

\begin{tabular}{cc}
\hline Samples & Cu (wt \%) \\
\hline TNFC $_{\text {R.O }}$ & $23.41 \pm 4.12$ \\
TNFC $_{Y . P}$ & $17.53 \pm 0.5$ \\
\hline
\end{tabular}




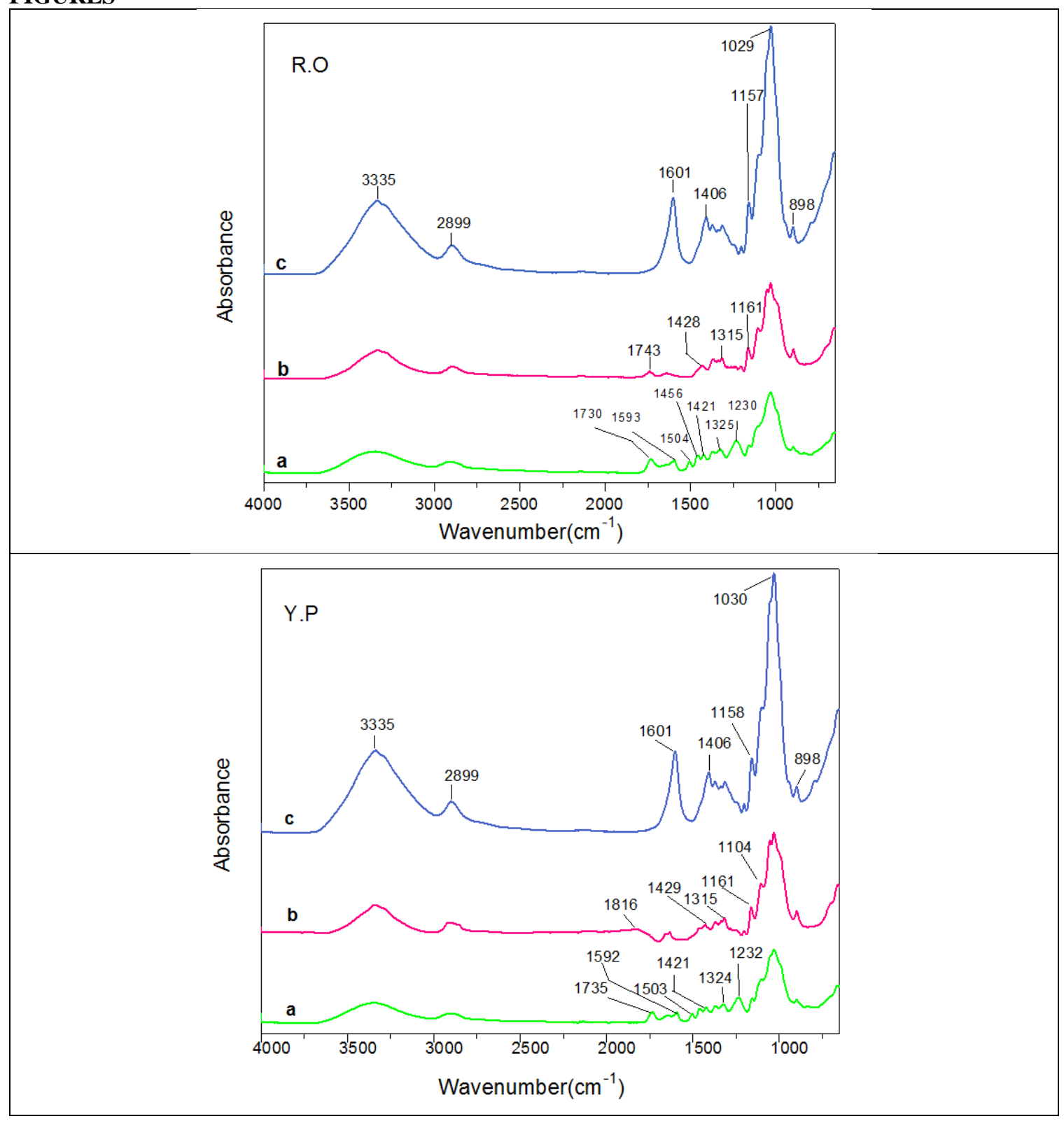

Figure 4.2 Upper figure: FT-IR spectra of: (a)Raw material, (b) Bleached, (c) Tempooxidized cellulose nanofibers (TNFC) of Red oak (R.O.); Lower figure: FT-IR spectra of: (a) Raw material, (b) Bleached, (c) Tempo-oxidized cellulose nanofibers (TNFC) of Yellow poplar (Y.P.) 


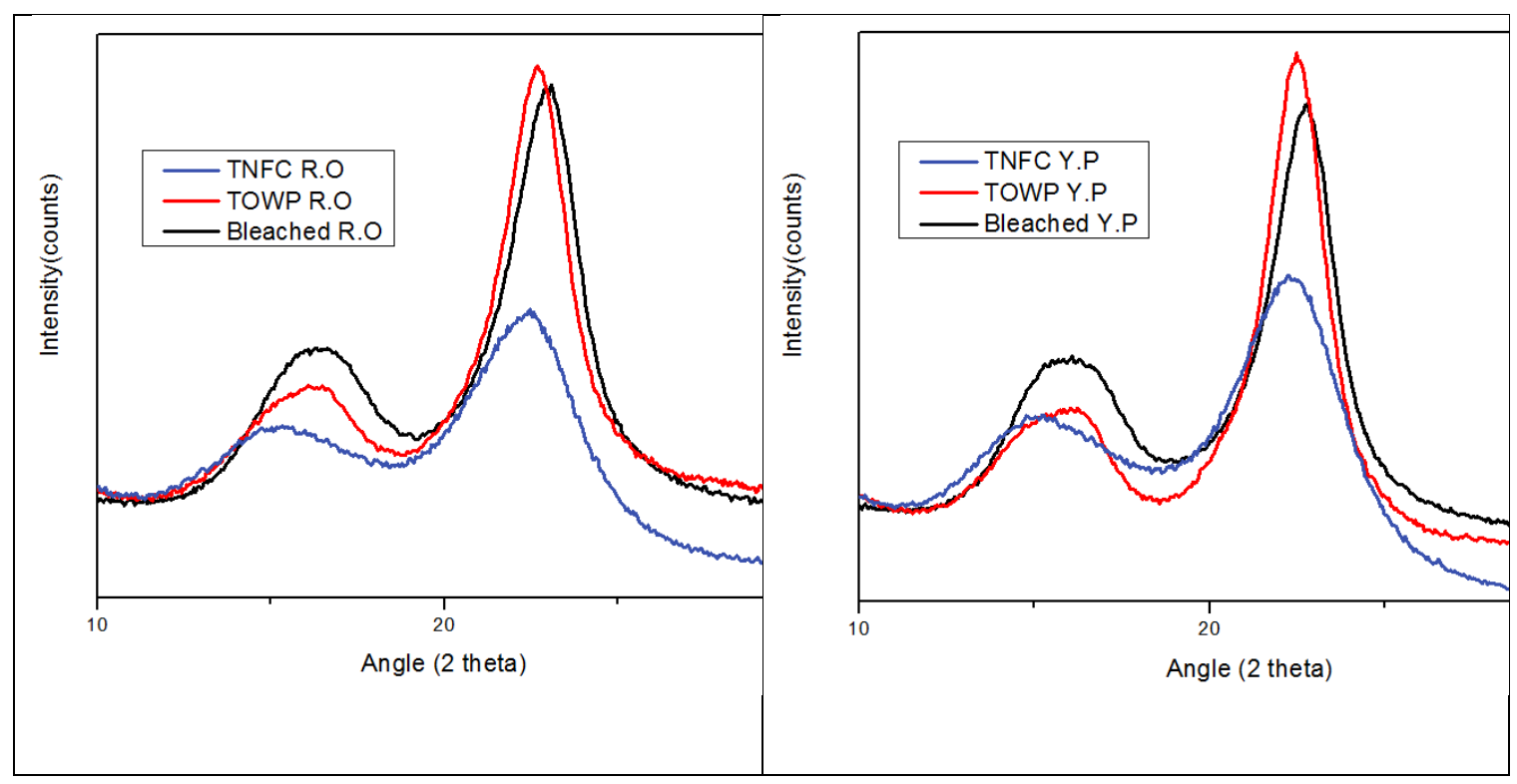

Figure 4.3 X-ray diffraction patterns of: Bleached, TOWP, and TNFC samples of R.O (left) and Y.P (right) 


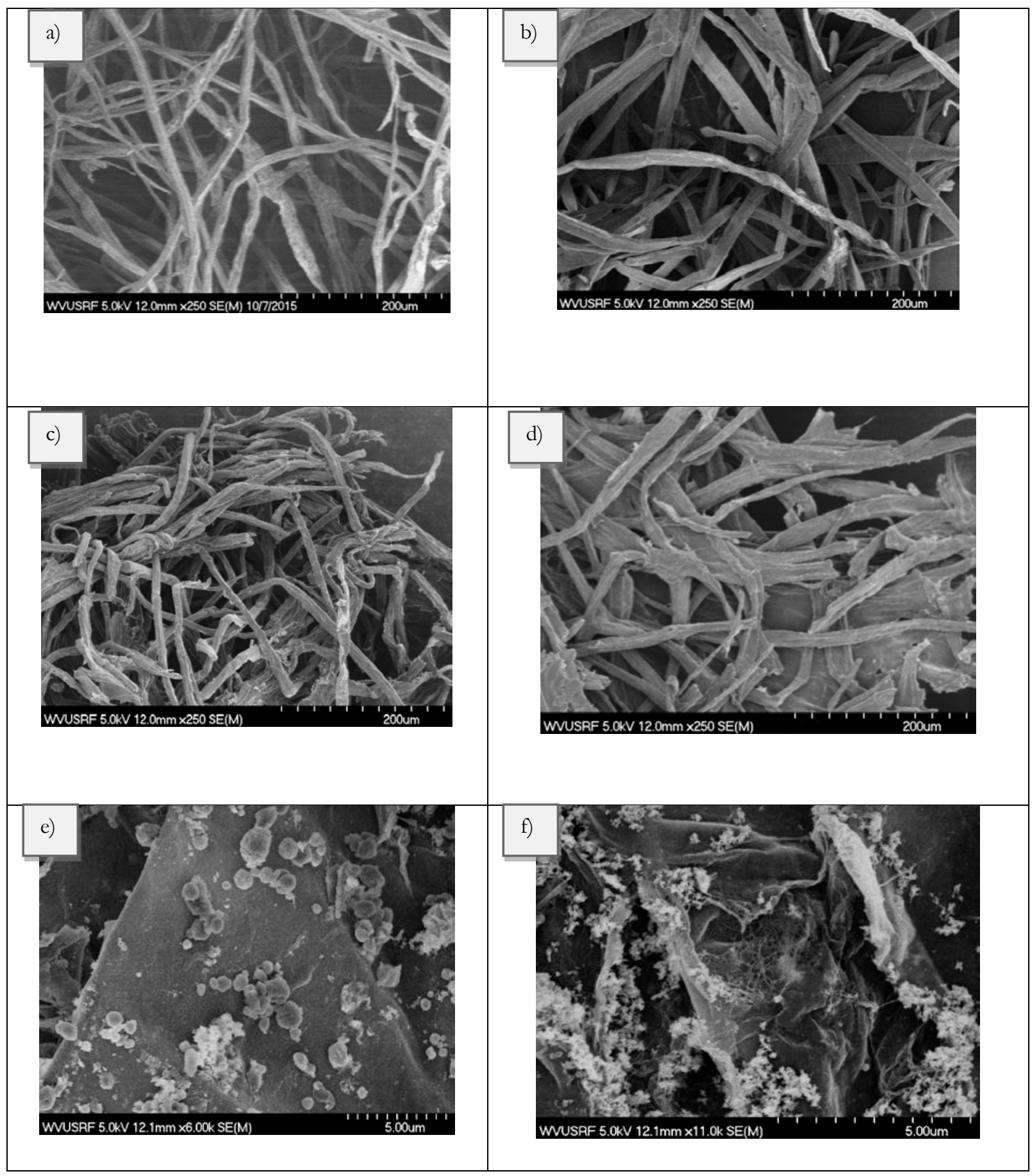

Figure 4.4 FE-SEM micrographs of the bleached R.O (a) and Y.P (b);TEMPO-oxidized wood pulp (TOWP) of R.O (c) and Y.P (d); TEMPO nanofibrillated cellulose and Copper $\left(\mathrm{TNFC}_{\mathrm{RO}}+\mathrm{Cu}\right)$ of R.O (e) and Y.P (f) samples. 


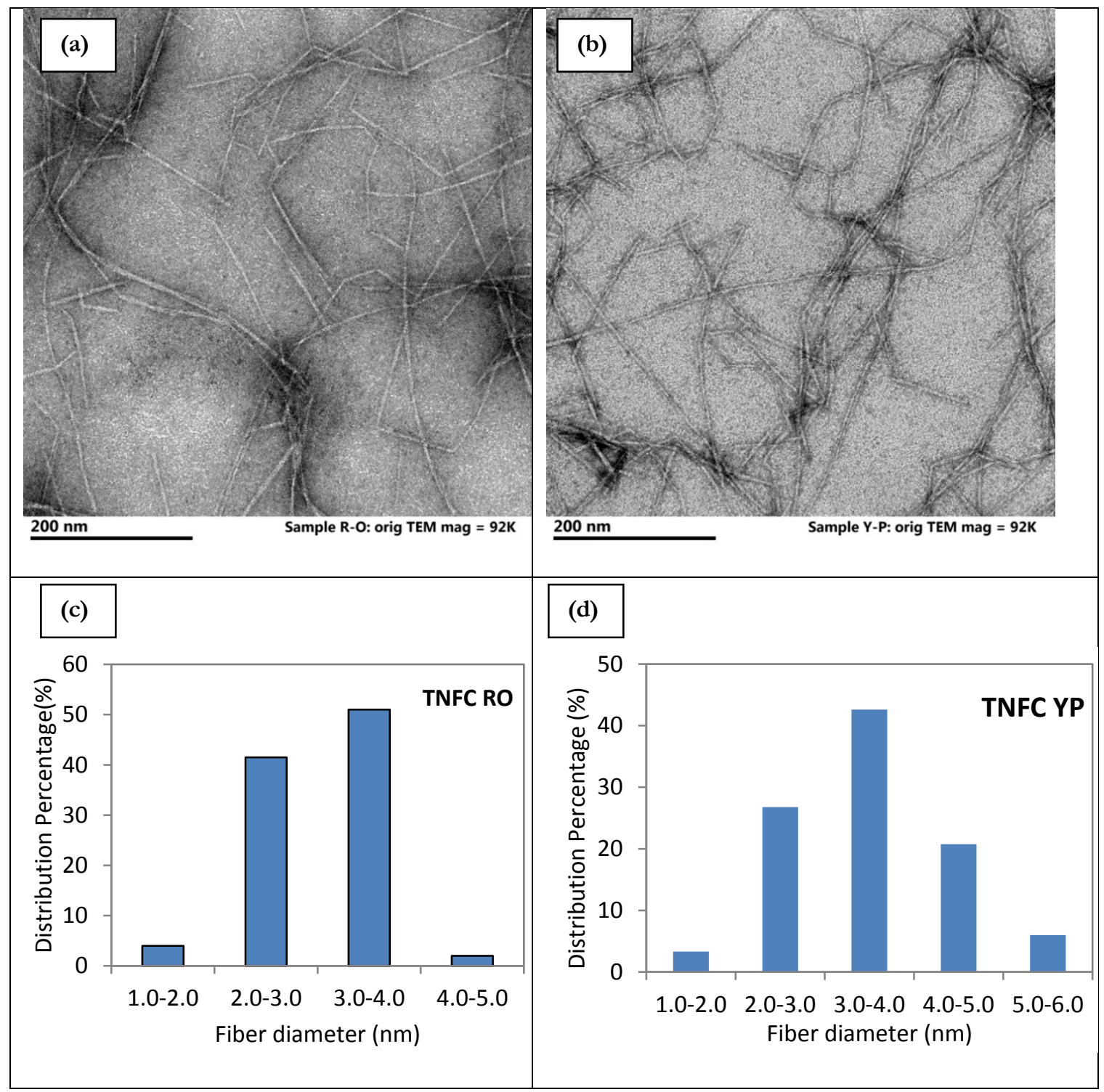

Figure 4.5 TEM images of TNFCR.o (a) and TNFCY.P (b) and their size distribution properties(c: R.O), (d: Y.P). 


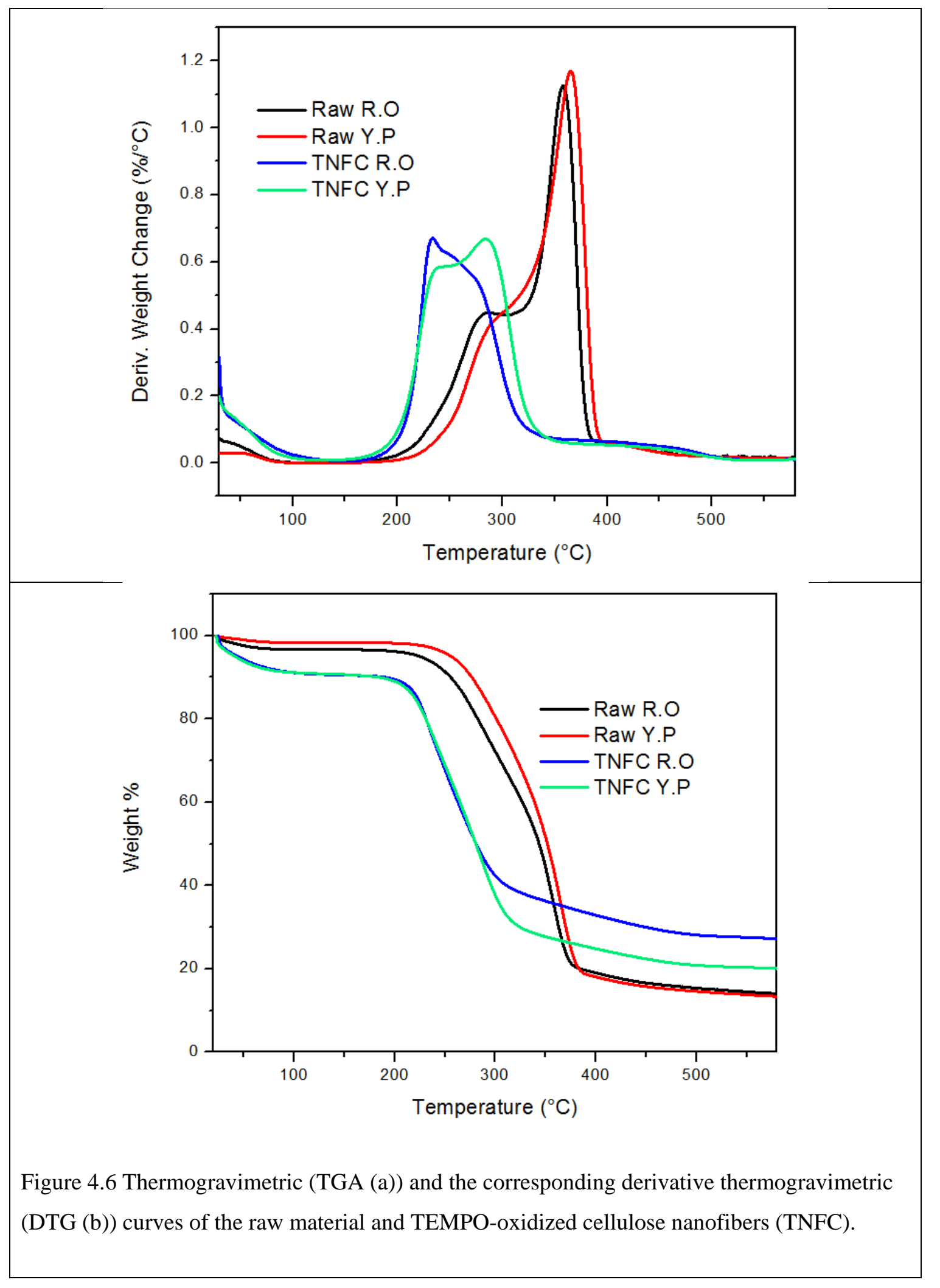




\subsection{References}

Alexander W, Goldschmid O, Mitchell R (1957) Relation of intrinsic viscosity to cellulose chain length. Degree of polymerization range below 400 Industrial \& Engineering Chemistry 49:1303-1306

Bufalino L et al. (2015) How the chemical nature of Brazilian hardwoods affects nanofibrillation of cellulose fibers and film optical quality Cellulose 22:3657-3672

Cao X, Ding B, Yu J, Al-Deyab SS (2012) Cellulose nanowhiskers extracted from TEMPOoxidized jute fibers Carbohydrate Polymers 90:1075-1080

Carlsson DO, Hua K, Forsgren J, Mihranyan A (2014) Aspirin degradation in surfacecharged TEMPO-oxidized mesoporous crystalline nanocellulose International Journal of Pharmaceutics 461:74-81

Chirayil CJ, Joy J, Mathew L, Mozetic M, Koetz J, Thomas S (2014) Isolation and characterization of cellulose nanofibrils from Helicteres isora plant Industrial Crops and Products 59:27-34

Davis MW (1998) A rapid modified method for compositional carbohydrate analysis of lignocellulosics by high $\mathrm{pH}$ anion-exchange chromatography with pulsed amperometric detection (HPAEC/PAD) Journal of Wood Chemistry and Technology 18:235-252

De Carvalho RA, Veronese G, Carvalho AJF, Barbu E, Amaral AC, Trovatti E (2016) The potential of TEMPO-oxidized nanofibrillar cellulose beads for cell delivery applications Cellulose 23:3399-3405

Deepa B et al. (2015) Utilization of various lignocellulosic biomass for the production of nanocellulose: a comparative study Cellulose 22:1075-1090

Dong H, Snyder JF, Williams KS, Andzelm JW (2013) Cation-induced hydrogels of cellulose nanofibrils with tunable moduli Biomacromolecules 14:3338-3345

Ferreira T, Rasband W (2012) ImageJ User Guide/IJ 1.46 r. Bioimage Informatics.

Hassanzadeh M, Sabo R, Rudie A, Reiner R, Gleisner R, Oporto GS (2017) Nanofibrillated Cellulose from Appalachian Hardwoods Logging Residues as Template for Antimicrobial Copper Journal of nanomaterials. Volume 2017, Article ID 2102987.

Isobe N, Chen X, Kim U-J, Kimura S, Wada M, Saito T, Isogai A (2013) TEMPO-oxidized cellulose hydrogel as a high-capacity and reusable heavy metal ion adsorbent Journal of Hazardous Materials 260:195-201

Jiang F, Hsieh Y-L (2013) Chemically and mechanically isolated nanocellulose and their self-assembled structures Carbohydrate Polymers 95:32-40

Kabir M, Wang H, Lau K, Cardona F (2013) Effects of chemical treatments on hemp fibre structure Applied Surface Science 276:13-23

Kalia S, Boufi S, Celli A, Kango S (2014) Nanofibrillated cellulose: surface modification and potential applications Colloid and Polymer Science 292:5-31 
Lani N, Ngadi N, Johari A, Jusoh M (2014) Isolation, characterization, and application of nanocellulose from oil palm empty fruit bunch fiber as nanocomposites Journal of Nanomaterials 2014:13

Le Troedec M. (2008) Influence of various chemical treatments on the composition and structure of hemp fibres Composites Part A: Applied Science and Manufacturing 39:514-522

Li J, Sevastyanova O, Gellerstedt G (2002) The relationship between kappa number and oxidizable structures in bleached kraft pulps Journal of Pulp and Paper Science (JPPS) 28:262-266

Liu D, Liu Y, Sui G (2016a) Synthesis and properties of sandwiched films of epoxy resin and graphene/cellulose nanowhiskers paper Composites Part A: Applied Science and Manufacturing 84:87-95

Liu P, Oksman K, Mathew AP (2016b) Surface adsorption and self-assembly of $\mathrm{Cu}$ (II) ions on TEMPO-oxidized cellulose nanofibers in aqueous media Journal of Colloid and Interface Science 464:175-182

Meng Q, Li H, Fu S, Lucia LA (2014) The non-trivial role of native xylans on the preparation of TEMPO-oxidized cellulose nanofibrils Reactive and Functional Polymers 85:142150

Missoum K, Belgacem MN, Bras J (2013) Nanofibrillated cellulose surface modification: a review Materials 6:1745-1766

Nacos M, Katapodis P, Pappas C, Daferera D, Tarantilis P, Christakopoulos P, Polissiou M (2006) Kenaf xylan-A source of biologically active acidic oligosaccharides Carbohydrate Polymers 66:126-134

Paukkonen H, Ukkonen A, Szilvay G, Yliperttula M, Laaksonen T (2017) Hydrophobinnanofibrillated cellulose stabilized emulsions for encapsulation and release of BCS class II drugs European Journal of Pharmaceutical Sciences 100:238-248

Poddar PK, Gupta A, Jamari SS, Kim NS, Khan TA, Sharma S, Aziz MAA (2015) Synthesis of nanocellulose from rubberwood fibers via ultrasonication combined with enzymatic and chemical pretreatments Asian Journal of Applied Sciences (ISSN: 2321-0893) 3

Rambabu N, Panthapulakkal S, Sain M, Dalai A (2016) Production of nanocellulose fibers from pinecone biomass: evaluation and optimization of chemical and mechanical treatment conditions on mechanical properties of nanocellulose films Industrial Crops and Products 83:746-754

Rodionova G, Eriksen Ø, Gregersen Ø (2012) TEMPO-oxidized cellulose nanofiber films: effect of surface morphology on water resistance Cellulose 19:1115-1123

Rodionova G, Saito T, Lenes M, Eriksen Ø, Gregersen Ø, Kuramae R, Isogai A (2013) TEMPO-mediated oxidation of Norway spruce and eucalyptus pulps: preparation and characterization of nanofibers and nanofiber dispersions Journal of Polymers and the Environment 21:207-214 
Sain M, Panthapulakkal S (2006) Bioprocess preparation of wheat straw fibers and their characterization Industrial Crops and Products 23:1-8

Saito T, Isogai A (2004) TEMPO-mediated oxidation of native cellulose. The effect of oxidation conditions on chemical and crystal structures of the water-insoluble fractions Biomacromolecules 5:1983-1989

Saito T, Kimura S, Nishiyama Y, Isogai A (2007) Cellulose nanofibers prepared by TEMPOmediated oxidation of native cellulose Biomacromolecules 8:2485-2491

Segal L, Creely J, Martin Jr A, Conrad C (1959) An empirical method for estimating the degree of crystallinity of native cellulose using the X-ray diffractometer Textile Research Journal 29:786-794

Sehaqui H, de Larraya UP, Liu P, Pfenninger N, Mathew AP, Zimmermann T, Tingaut P (2014) Enhancing adsorption of heavy metal ions onto biobased nanofibers from waste pulp residues for application in wastewater treatment Cellulose 21:2831-2844

Sehaqui H, Gálvez ME, Becatinni V, cheng Ng Y, Steinfeld A, Zimmermann T, Tingaut P (2015) Fast and Reversible Direct CO2 Capture from Air onto All-Polymer Nanofibrillated Cellulose. Polyethylenimine Foams Environmental Science \& Technology 49:3167-3174

Shin NH, Stromberg B Xylan's impact on eucalyptus pulp yield and strength-Myth or reality. In: International Colloquium on Eucalyptus Pulp, 2007.

Soni B, Mahmoud B (2015) Chemical isolation and characterization of different cellulose nanofibers from cotton stalks Carbohydrate polymers 134:581-589

Sun X, Xu F, Sun R, Fowler P, Baird M (2005) Characteristics of degraded cellulose obtained from steam-exploded wheat straw Carbohydrate Research 340:97-106

Syverud K, Chinga-Carrasco G, Toledo J, Toledo PG (2011) A comparative study of Eucalyptus and Pinus radiata pulp fibres as raw materials for production of cellulose nanofibrils Carbohydrate Polymers 84:1033-1038

Wicaksono R, Syamsu K, Yuliasih I, Nasir M, Street K (2013) Cellulose nanofibers from cassava bagasse: Characterization and application on tapioca-film Cellulose 3:79-87

Wulandari W, Rochliadi A, Arcana I Nanocellulose prepared by acid hydrolysis of isolated cellulose from sugarcane bagasse. In: IOP Conference Series: Materials Science and Engineering, 2016. IOP Publishing, p 012045

Zhong T, Oporto GS, Jaczynski J, Jiang C (2015a) Nanofibrillated cellulose and copper nanoparticles embedded in polyvinyl alcohol films for antimicrobial applications Biomed Research International Article ID 456834.

Zhong T, Oporto GS, Peng Y, Xie X, Gardner DJ (2015b) Drying cellulose-based materials containing copper nanoparticles Cellulose 22:2665-2681 
CHAPTER 5: PREPARATION OF NANOCELLULOSE USING IONIC LIQUIDS A REVIEW

\begin{abstract}
In recent decades, dependence on fossil fuels resources has shifted into the derivation of cellulose based materials to replace the non-renewable resources. Cellulose is the most abundant bio-based polymer available in large amounts anywhere on earth and is one of the promising renewable and sustainable resources.
\end{abstract}

Cellulose nanofiber, which is obtained from cellulose, is creating a revolution in bio-based materials for diverse applications. cellulose nanofibers (NFC) is equipped with various superior characteristics including nanoscale dimension, high surface area, unique optical properties, high crystallinity, and stiffness together with the biodegradability and renewability. Its nano-scale dimensions and its capacity to form a strong entangled nanoporous network have encouraged the emergence of new high-value applications. It is worth noting that chemical surface modification of this material can be a key factor to achieve a better compatibility with matrices. In order to increase the compatibility in different matrices or to add new functions, surface chemical modification of NFC appears to be the prior choice to conserve its intrinsic nanofibre properties.

Due to the drawbacks associated with the current methodologies used to dissolve and process cellulose, environmentally friendly and more efficient solvents are required. In the last decade, ionic liquids have emerged as effective and green solvents, mainly due to their high thermal and chemical stability, nonflammable nature and miscibility with many other solvent systems. These solvents are often fluid at room temperature, and consist entirely of ionic species. In this report, a short literature review of Ils, their benefits, and drawbacks together with some examples of methods of separation of nanocellulose using ionic liquids would be discussed. 


\subsection{Problem statement}

Cellulose is the most abundant biopolymer available on earth. Its abundance, biodegradability, recyclability and renewability make cellulose one of the best alternative raw materials to oil-based sources. The last decade has focused on obtaining efficient materials from cellulose fibers with an exponential interest in nano-scaled cellulose-based elements (Missoum et al. 2012). A biomass pretreatment step is necessary to ensure the separation of cellulose component from the tight bond of polymeric constituents (cellulose, hemicellulose, and lignin) in lignocellulosic biomass. The main intention of this fractionation treatment is to increase the accessibility of cellulose fiber to chemical attack prior to mild hydrolysis of isolated cellulose, by cleaving the ether bond between glucose chain in order to produce nanosize cellulose intermediate. However, biomass fractionation is a very complex process as high recovery of polysaccharides (cellulose, hemicellulose, and lignin) is required so that all three components can be fully converted into useful end products.

At a first glance, cellulose looks very simple. Nevertheless, dissolution of cellulose is in fact quite complicated. Many factors contribute to make cellulose insoluble in most common solvents. Native cellulose has a high DP, which leads to a decrease in solubility due to a reduction in entropic gain in the dissolution process. Glucose, cellobiose, or any oligomer of cellulose structure upto a DP around ten are soluble in simple solvents, such as water. However, cellulose is not, so the length of the polymer is obviously a relevant factor (Liebert et al. 2008).

An additional challenge is the interactions between the cellulose molecules. Three hydroxyl groups per AGU make complex patterns of hydrogen bonds possible. To break these bonds, solvents with a high capacity for hydrogen bonding are necessary. Water alone cannot dissolve cellulose, yet both water - water, carbohydrate -water and carbohydrate carbohydrate hydrogen bond strengths are around $5 \mathrm{kcal} / \mathrm{mol}$ (Olsson 2014). Thus, hydrogen bonding is not the only relevant factor for a suitable solvent but other types of interaction must be taken into consideration. As shown in Figure 5.1, hydrogen bonds arrange cellulose chains in sheets, but stacking of these sheets into the three dimensional structure of the cellulose material involves hydrophobic interactions. 

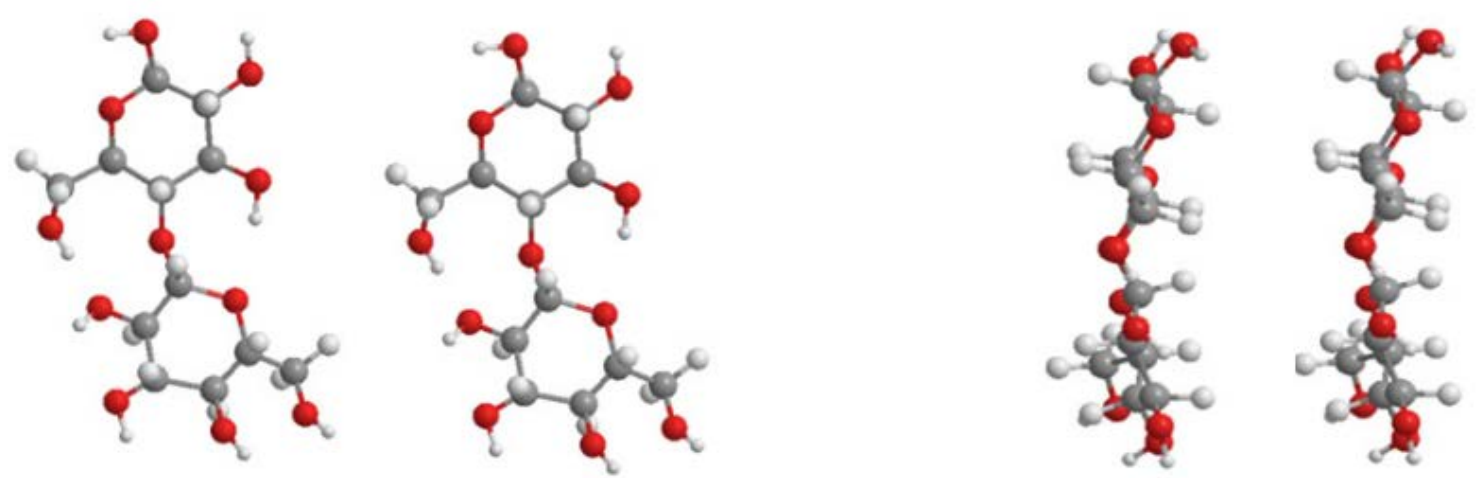

Figure 5.1 Cellulose simplified as a cellobiose. Equatorial hydrogen bonds arrange cellulose chains in sheets that are stacked onto each other in three-dimensional crystals by means of hydrophobic interactions (Olsson 2014).

Swelling of cellulose is a process in which a liquid molecule penetrates the fiber structure by interacting with the cellulose polymer to a certain extent, leaving the volume and physical properties of the cellulose significantly changed, although the solid or semi-solid state remains. This occurs, for instance, in water and dilute lye. Dissolution, on the other hand, completely destroys the supramolecular structure (e.g. the fiber) due to a superior interaction between the solvent and the polymer, and results in a clear solution in which the polymers are molecularly dispersed. In the last ten years, research on cellulose solubility has, to a large extent, been dominated by research on ionic liquids.

Processing and derivatization of cellulose are also rather difficult due to the complex structure of biopolymeric network and presence of numerous noncovalent interactions between cellulose molecules. Besides that, partially crystalline, highly ordered structure as well as the stiff molecules results from the closely-packed chains via extended network of inter- and intramolecular hydrogen bonds and rigid linkages lead to insolubility of cellulose in water and other common organic solvents (Kokol et al. 2015; Xu et al. 2010). In this case, these constitute a serious problem for cellulose dissolution and activity as well as obstruct the application of cellulose.

A number of methods such as acid hydrolysis, steam explosion, mechanical process, TEMPO-mediated oxidation, thermal hydrolysis and pyrolysis have been used to breakdown the cellulose molecule into nanocellulose. However, these methods not only destroy the 
pristine structure of cellulose but also added impurities such as halide and metal ions into the final products and are difficult controllable processes as there are propensity of over degradation of cellulose (Tang et al. 2011).

Chemical treatments are the most popular pretreatment technologies in the isolation of cellulose fibers to nanocellulose (Lee et al. 2014). Based on the type of treatment, several types of nanocellulose can be found in the literature: the cellulose nanocrystals (or whiskers or $\mathrm{CNC}$ ) obtained by acid hydrolysis of cellulose and cellulose microfibrils or nanofibrils (NFC) obtained by mechanical treatments usually combined with enzymatic and/or chemical pre-treatment of cellulosic materials (Missoum et al. 2013). Cellulose microfibrils (MFC, NFC) firstly obtained by Herrick et al. and Turbak et al. in 1983 using mechanical disintegration of wood pulp. The diameter of nanofibrils is in the range of 10 to $50 \mathrm{~nm}$, whereas the typical length is several micrometers. The obtained NFCs show improved properties (e.g. mechanical, barrier) and allow efficient bionanomaterials to be made. Unfortunately, NFCs tend to form an aqueous gel at very low concentration (2\% wt) due to their important specific surface area and consequently the high number of hydrogen bonds arising from surface hydroxyl groups. This feature is the main drawback of their use in several applications, such as coating formulations (low solid content and high viscosity). The aggregation or the film-forming capacity of NFCs in their dried form is another inconvenience, mainly for composite applications. To overcome these issues, different solutions are studied but the most developed are the surface chemical modification, transforming hydroxyl groups into other functions thus limiting (or even totally avoiding) the formation of hydrogen bonds(Missoum et al. 2012).

Surface treatment of NFCs is important to tailor NFCs surfaces for application in specific polymer matrices, in terms of both compatibility and controlling their aggregation and is still an ongoing research(Croitoru and Patachia 2016).

Different techniques can be used for such NFC modifications as trimethylsilylation, ring opening polymerization of poly-(3-caprolactone), cerium induced grafting, surface 
acetylation, carboxymethylation. To perform these reactions, large quantities of organic solvents as liquid media for chemical reactions, for extraction or in the formulation are overused. In most cases, these dispersing organic media are hazardous to health, flammable and volatile.

The choice of solvent is paramount as it will determine the greenness of the final material. So-called "green solvents" should: (i) be non-toxic and easily biodegradable, thereby providing safe work conditions, (ii) be efficiently recycled in order to avoid their propagation in ecosystems and (iii) integrate a low energy production cycle. The last two points have the additional benefit of making the production cycle more cost-effective (Duchemin et al. 2009).

To date, numerous solvent systems have been identified such as $\mathrm{N}$-methylmorpholine oxide (NMMO), N,N-dimethylacetamide/ lithium chloride (DMAc/LiCl), N,Ndimethylformamide/nitrous tetroxide (DMF/N2O4), dimethyl sulfoxide/tetrabutylammonium fluoride (DMSO/TBAF), 1,3-dimethyl-2-imidazolidinone/ lithium chloride ( $\mathrm{DMI} / \mathrm{LiCl}$ ) and some molten salt hydrates such as $\mathrm{LiClO} 4 \cdot 3 \mathrm{H} 2 \mathrm{O}$, LiSCN·2H2O and other aqueous metal complexes are able to dissolve cellulose effectively (Tian et al. 2014; Xu et al. 2013). Those methods and solvents systems proposed are worthy to mention several shortcomings might be occurred including toxicity, high cost, volatility, generation of poisonous gas, difficulty in solvent recovery and process instability (Lan et al. 2011). In addition, multistep treatment and complicated separation process are usually required, followed by prolonged reaction time up to several hours to days.

To limit the use of such organic solvents, a novel type of "green”" solvents has been studied during these past decades, i.e.: the ionic liquids (ILs) (Missoum et al. 2012; Oksman et al. 2016).

ILs are capable to solubilize cellulose by causing the disruption to the hydrogen bonding network of cellulose. Besides that, atmospheric solvent loss and flammability hazard can be minimized via the application of ILs because they have low vapor pressure and better thermal 
stability. Moreover, they are ease of recovering and the ability to recycle and reusable contributes to the lower operating cost of the catalytic process. In overall, the substitution of acids by ILs can act as an environmentally friendly approach to synthesis the nanocellulose (Tan 2016).

\subsection{Ionic Liquids (ILs)}

As early as in the 1930s, it was found that liquefied quaternary ammonium salts, alone or diluted in suitable solvents, can dissolve cellulose (Graenacher 1934). This was the first successful direct dissolution of cellulose in organic salt. However, at that time, these findings did not attract much attention. It took almost 70 years for this idea to blossom. In the early 2000s, it was found that neat 1-butyl-3-methylimidazolium chloride (BMIMCl) can dissolve cellulose quickly and without derivatization (Swatloski et al. 2002). This finding would set the spark to a formidable explosion in the so called ionic liquid research related to cellulose.

Ionic liquids or “'molten salts"' are in general defined as liquid electrolytes composed entirely of ions. One of the most important features of ILs is their non-measurable vapor pressure. They are defined as “green" solvents mainly because of the absence of volatile organic compounds (VOC) emission. Also in terms of inhalation and vapor of the solvent, the risk is limited in comparison to other volatile organic solvents in spite of IL toxicity. Furthermore, ILs have other attractive properties, such as high chemical and thermal stability nonflammability, as well as high ionic conductivity(Blake et al. 2006; Chiappe and Pieraccini 2005; Zhang et al. 2006). They could be also easily recyclable and reused due to their low melting point(comprising " $-60^{\circ} \mathrm{C}$ to $+60^{\circ} \mathrm{C}$ ), just by decreasing the temperature or by distillation. There is a wide variety of ILs, each of them is composed of a cation (e.g. imidazolium or pyridinium salts) and an anion (e.g. chloride, tetrafluoroborate, hexafluorophosphate.).

Ionic liquids (ILs) represent chemicals simply defined as organic salts with a melting point below $100^{\circ} \mathrm{C}$. If an ionic liquid has a melting point below room temperature, it is often called room temperature ionic liquid (RTIL)(Olsson 2014). 
ILs consist entirely of ions (when adding a molecular co-solvent, the term "electrolyte solution” is more applicable (Hummel et al. 2015) where one or both the ions are large, and the cation has a low degree of symmetry. These factors tend to reduce the lattice energy of the crystalline form of the salt, and hence lower the melting point (Earle and Seddon 2000),thus forming stable liquids at temperatures below 100C (Tan 2016).

In most ionic liquids bulky organic cations and weakly coordinating anions are used to create molten salts at low temperature. Also, the alkyl chain length of the cation adds a conformational entropic drop in the melting point(Thunberg 2015).

Although ionic liquids (ILs) have been known for many decades, significant research activity in this field started only in the early 1990s. Their unique properties and a broad spectrum of application possibilities have promoted intensive research activity (Hummel et al. 2015).

Ionic liquids come in two main categories, namely simple salts (made of a single anion and cation) and binary ionic liquids (salts where equilibrium is involved). For example, [EtNH3][NO3] is a simple salt whereas mixtures of aluminum(III) chloride and 1,3dialkylimidazolium chlorides (a binary ionic liquid system) contain several different ionic species, and their melting point and properties depend upon the mole fractions of the aluminum(III) chloride and 1,3-dialkylimidazolium chloride present.

Ionic liquids have been described as designer solvents, and this means that their properties can be adjusted to suit the requirements of a particular process. Properties such as melting point, viscosity, density, and hydrophobicity can be varied by simple changes to the structure of the ions(Earle and Seddon 2000) .

The cations of ILs are often featuring an aromatic or cyclic structure and long alkyl chain. Common cations are alkylimidazolium, alkylpyridinium and quaternary ammonium based. Whereas the anions of ILs can be varied from chloride, formate, acetate, bromide, tetrafluoroborate, hexafluorophosphate or methanesulfonate (Gericke et al. 2012). Some common examples of cations and anions for ILs with their molecular structures are presented 
in Figure 5.2. In fact, various combinations of cations and anions will contribute to the variety types of ILs.As such, task-specific ILs (TSILs) are arise for particular applications with desirable properties.

The dissolution mechanism of cellulose in ionic liquids has long been argued to be all about hydrogen bond interactions. Several studies have confirmed that the anion of the ionic liquid penetrates the native cellulose structure and dissembles it by means of competitive hydrogen bonding(Olsson 2014) . The bonding patterns depend on which anion that is used. Some, like acetate, can bond not only to one but possibly to two sites along the cellulose chain (Rabideau et al. 2014). Others, like chloride, have instead been suggested to interfere with the cellulose disintegration mechanism and actually hinder further dissolution by bridging between two adjacent cellulose chains (Youngs et al. 2011).

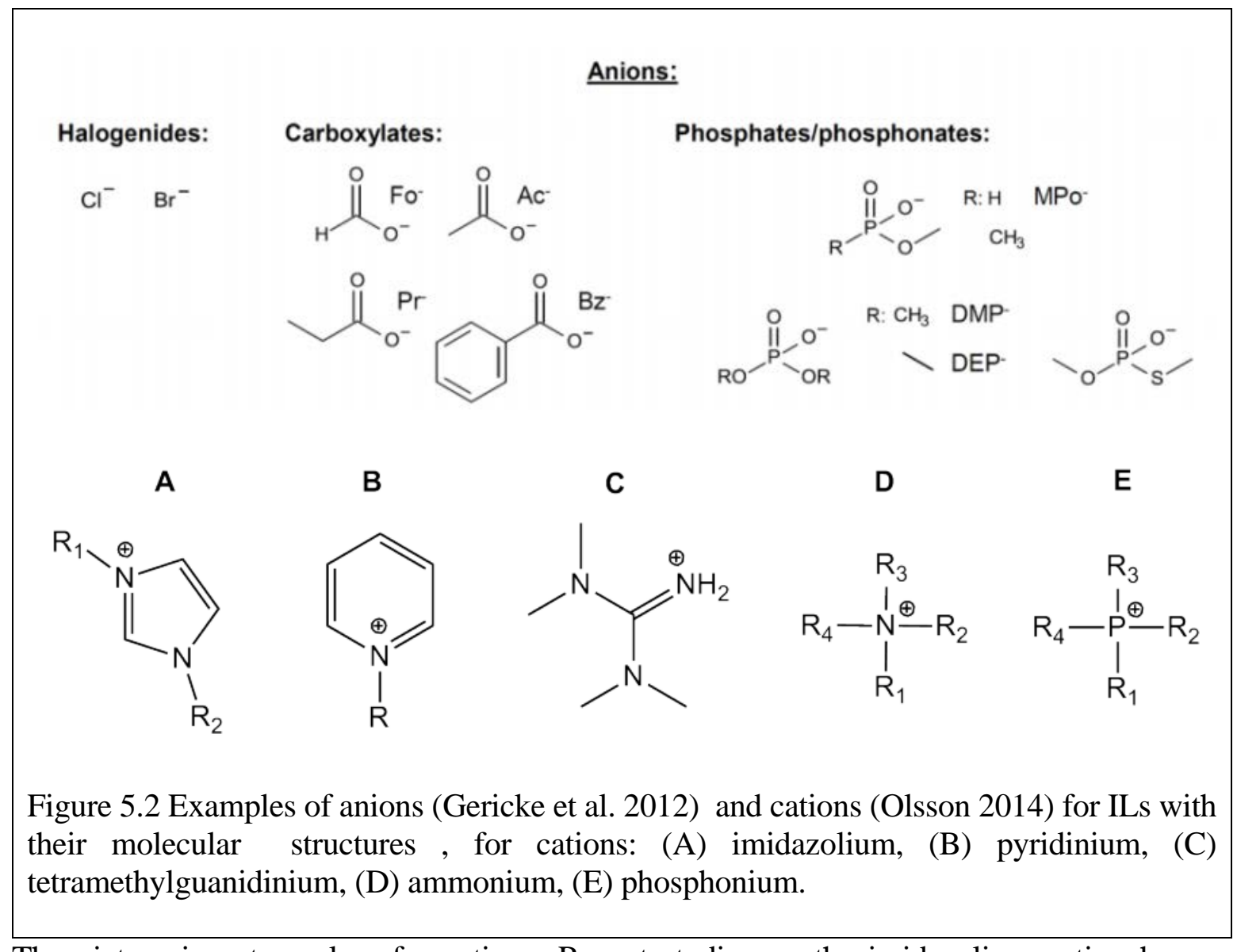

The picture is not as clear for cations. Recent studies on the imidazolium cation have explained, in detail, the effect of cation asymmetry and show effects on glass temperature, 
viscosity and ion mobility (Zheng et al. 2011). Although the common view now is that it is the anion that is mainly responsible for the dissolution of cellulose, the number of carbons in the alkyl chains of the imidazolium cations actually influences the dissolution ability of the corresponding ionic liquid. Longer alkyl chains in the cation leads to a decreased cellulose solubility (Vitz et al. 2009).

Cellulose is the most widely investigated lignocellulosic biomass component for IL dissolution(Reddy 2015). The first attempt to use ionic liquids for processing cellulose can be found in a patent from 1934. Graenacher used N-ethylpyridinum as the cation and chloride as the anion to dissolve cellulose (Thunberg 2015). Large scale dissolution of cellulose with $\mathrm{N}$-ethylpyridinium chloride never saw application. Renewed interest in cellulose processing using molten salts occurred in the 2000s when imidazolium based ionic liquids were shown to readily dissolve cellulose at room temperature(Thunberg 2015).

Swatloski et al. have shown that 1-butyl-3-methylimidazolium chloride (BMIMCl) can dissolve up to $25 \%$ weight cellulose with microwave heating. Swatloski and co-workers also demonstrated that the dissolution of cellulose in BMIMCl was mainly attributed to the interaction between anions of IL with cellulose (Swatloski et al. 2002).

The capability to dissolve large amounts of cellulose has also been shown in other imidazolium based ionic liquids (Kosan et al. 2008).

$\mathrm{Li}$ et al. for first time coupled pretreatment by 1- butyl-3-methylimidazolium chloride ([Bmim]Cl) as an ILs to isolate NFC from sugarcane bagasse. The ILs dissolved cellulose and easily passed through homogenizer without clogging. Afterward, cellulose was precipitated by adding water and was regenerated the NFC by freeze drying. They found that solubilization of cellulose was affected by the reaction temperature, the power of microwave and weight ratio of cellulose to Ils(Li et al. 2012).

The regeneration of the dissolved cellulose in an amorphous form was also demonstrated through the use of anti-solvents (such as water) which were IL soluble. This work showcased 
the ability of ILs to not only dissolve cellulose but also to modify its crystalline structure (Reddy 2015).

The cellulose dissolution in IL is facilitated as cellulose is swelled due to the separation of hydroxyl groups of different cellulose chains. In overall, cellulose dissolution in IL can be explained due to the interaction between anions of IL with the hydroxyl groups of cellulose, though the IL cations might have a minor role (Figure 5.3)(Tan 2016).

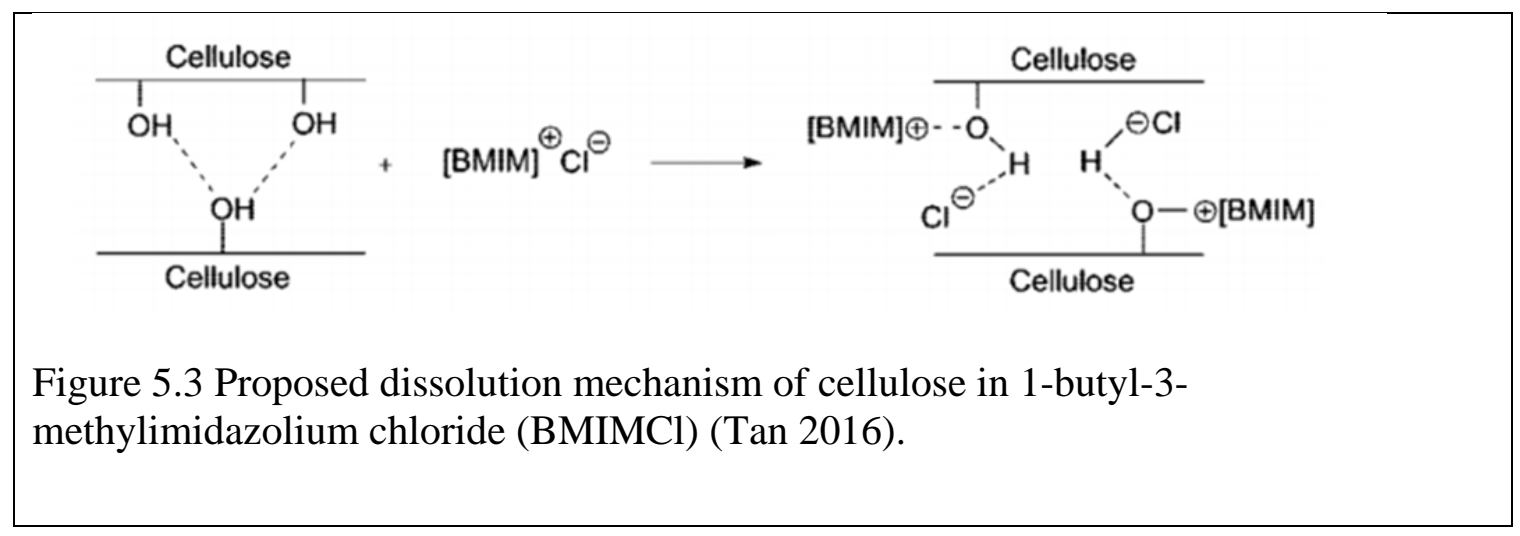

Thus, hydrogen bonding acceptor ability (basicity) of anions of IL has an important role in the solubility of cellulose. In general, anions with good hydrogen bond acceptors are more effective to dissolve cellulose. Examples are chloride, formate, acetate, phosphate and phosphonate anions. The solubility of cellulose in ILs is dependent on the hydrogen bond accepting ability of the anions. The higher the hydrogen bond basicity or accepting ability, the higher the ability of that particular anion of ILs to dissolve cellulose(Tan 2016). It was found out that chloride, as a small hydrogen bond acceptor, was the most effective anion to dissolve cellulose in comparison to large, non-coordinating anions (Isik et al. 2014).

To date, only a few ILs are known that serve as cellulose solvents. These so-called first generation spinning ILs typically comprise an imidazoliumcation with chloride as the anion. However, their high melting point and the corrosive character of halides toward metal processing equipment have promoted the implementation of alternative anions such as acetate and dialkylphosphate(Hummel et al. 2015). Figure 5.4 gives an overview of the structure of anions (a,b,c,d) and cations(1,2,3). 


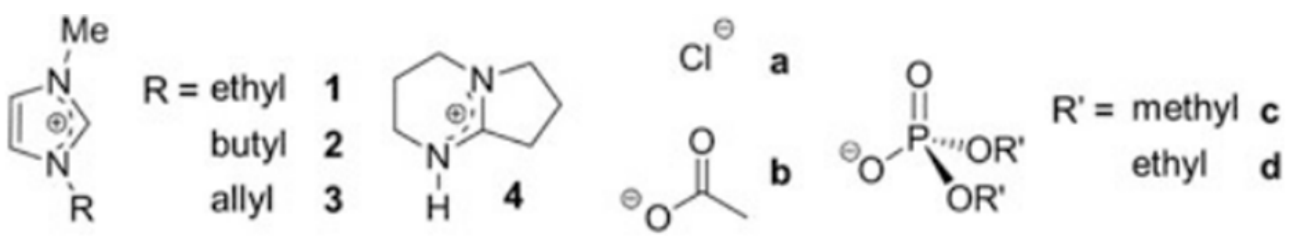

Figure 5.4 Constituent ions of ILs used for fiber spinning:11-ethyl-3methylimidazolium,21-butyl-3-methylimidazolium, 3 1-allyl-3-methylimidazolium, 4 1,5-diazabicyclo[4.3.0]non-5-enium (Hummel et al. 2015).

Also shown are typical ions, including chloride, acetate, dimethylphosphate, and diethylphosphate (a, b, c, and d, respectively). ILs are denoted in the text by a digit representing a cation (1-4) followed by a letter (a-d) representing the counter-ion (Hummel et al. 2015).

ILs makes the catalytic sites highly access the $\beta$-glycosidic bonds, which facilitates the reaction of biomass fractionation and hydrolysis of cellulose. An ideal ILs for lignocellulosic biomass pretreatment process should possess the following: (i) high dissolution capacity for different component by varying organic cation; (ii) low melting point; (iii) low viscosity; (iv) low/no toxicity; and (v) high stability (Lee et al. 2014). Figure 5.5 shows possible routes after dissolution of cellulose using Ils.

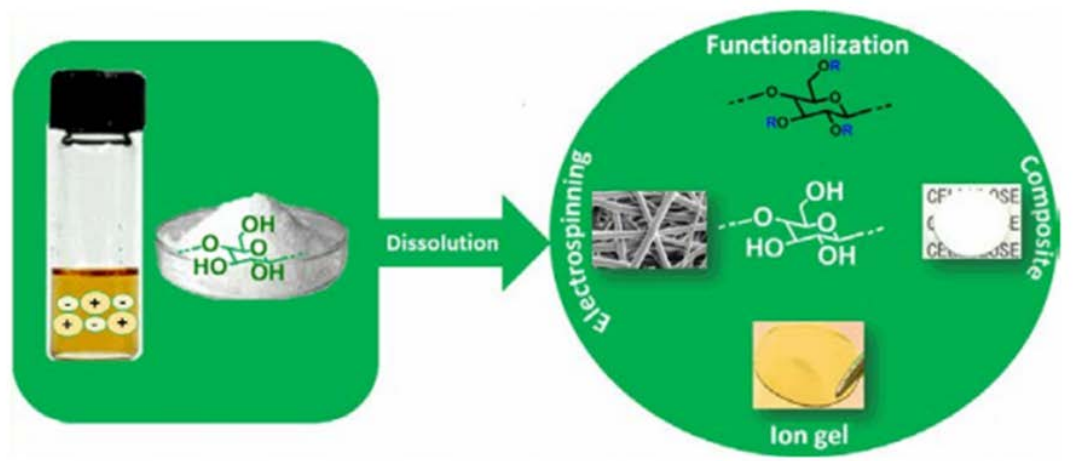

Figure 5.5 Possible materials and technologies generated from cellulose dissolved in ionic liquids (Isik et al. 2014). 


\subsubsection{Advantages of ILs}

Traditional cellulose dissolution processes, including the cuprammonium and xanthate processes are often cumbersome or expensive and require the use of unusual solvents, typically with high ionic strength and use relatively harsh conditions. Moreover, these processes sometimes cause serious environmental problems because these solvents cannot be recovered and reused. In recent years, the “green”' comprehensive utilization of cellulose resources has drawn much attention from the governments and researchers. Traditional cellulose extraction and dissolution processes are facing challenges because of the energy and environmental problems. Therefore, to make full use of cellulose resources, it is necessary to develop “green”' cellulose extraction methods and suitable cellulose dissolution approaches (Zhu et al. 2006).

As they are made up of at least two components which can be varied (the anion and cation), the solvents can be designed with a particular end use in mind, or to possess a particular set of properties. Hence, the term "designer solvents" has come into common use (Earle and Seddon 2000).

Other than biomass degradation to isolate cellulose product, ILs pretreatment could reduce the crystallinity of cellulose to amorphous nature. Cellulose in the form of fibrils poorly hydrolyses or depolymerize under mild condition due to its intermolecular hydrogen linkages between polysaccharide chains.Ils is capable of disrupting the hydrogen bonds by forming another hydrogen bond between anion of IL with cellulose (sugar hydroxyl protons) in a 1 : 1 ratio. This will break up the cellulose hydrogen bonded structure, thus decreasing the compactness of cellulose and making it more amorphous and susceptible to depolymerisation process (Lee et al. 2014).

ILs can dissolve cellulose, hemicellulose, and lignin under considerably mild conditions without degrading the chain's structure(Lee et al. 2014).

A positive safety aspect of ionic liquids is that they are usually not flammable because of its negligible' volatility under ambient conditions as most volatile organic solvents are. Also, 
the polarity of ionic liquids is mainly a function of the anion, which can be altered. Another interesting property of ionic liquids is that the vapor pressure is zero or at least negligible. The non-volatility of ionic liquids effectively reduces the risk for environmental release and contamination, in contrast to volatile organic solvents. Ionic liquids have been called green solvents because of these properties (Thunberg 2015). In this context, They have a host of potential applications from thermal energy storage to pharmaceuticals(Reddy 2015). Also in terms of inhalation and vapor of the solvent, the risk is limited in comparison to other volatile organic solvents(Missoum et al. 2012).

Ionic liquids have been called designer solvents because of the many combinations of cations and anions possible in their structure(Thunberg 2015).

Using a "green"' solvent (ionic liquid) could give rise to an efficient grafting of nanoscaled cellulosic substrates without affecting their morphological properties. Moreover, the ionic liquids can be recovered and reused after cellulose regeneration due to their low melting point. Various methods, such as evaporation, ionic exchange, reverse osmosis and salting out are used to recover the ionic liquid (Zhu et al. 2006). Several techniques to assess the quality of the recycled IL were performed and showed that the resulting recycled solvent is quite pure and ready to be used for the next cycle of chemical grafting(Missoum et al. 2012).

Furthermore, ILs have other attractive properties, such as high chemical and thermal stability, as well as high ionic conductivity (Missoum et al. 2012).

The Ionic liquid can dissolve both carbohydrates and lignin, which disrupt the intricate network of noncovalent interactions between these polymers. This treatment can reduce lignin content and cellulose crystallinity(Lee et al. 2014).

With the potential number of ILs that can be synthesized estimated as at least a billion, coupled with the tunable nature of their physicochemical properties, the opportunities for designing task-specific ILs are abundant (Reddy 2015). 
The dissolution of the lignocellulosic biomass in the IL pretreatment allows for the cellulose and other lignocellulosic components to be accessible to external reagents and catalysts (chemical and biological) dissolved or dispersed in the same medium for conversion processes (Reddy 2015).

Dissolution of cellulose with ionic liquids allows the comprehensive utilization of cellulose by combining two major green chemistry principles: using environmentally preferable solvents and bio-renewable feedstock(Frone et al. 2011).

\subsubsection{Dis-advantages of ILs}

Ionic liquids that are able to dissolve cellulose have high viscosity, and dissolution of cellulose makes the ionic liquid/cellulose solution even more viscous. One approach to decreasing viscosity and improve dissolution dynamics has been adding a co-solvent to the ionic liquid. For instance, adding N-methylimidazole, the precursor for the methylimidazolecation, as co-solvent in methylimidazolium ionic liquids decreases dissolution time and reduces the viscosity of cellulose/EMIMAc solutions (Olsson et al. 2014). The molecular structure for common imadizoliumcations in ionic liquids for cellulose dissolution is shown in Figure 5.6.
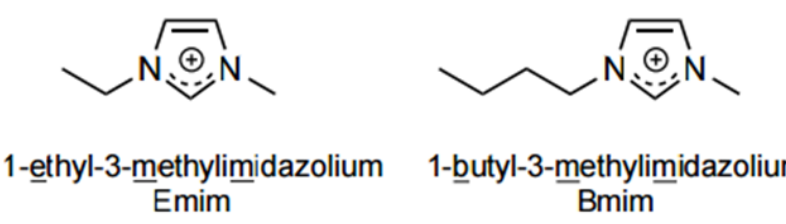

Bmim

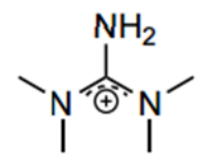

Figure 5.6 Common ionic liquid cations used for cellulose dissolution. 1-ethyl-3methylimidazolium acetate is abbreviated EMIMAc (Thunberg 2015).

The alleged non-derivatizing property of EMIMAc as cellulose solvent has been challenged and EMIMAc has been shown to react with cellulose end-groups, and also to react with cellulose hydroxyl groups under certain conditions (Thunberg 2015).

Additionally, the upscaling of ILs from being mere laboratory curiosities to wide-scale commercially viable technologies has remained to date a significant challenge. This challenge is primarily a result of the high costs of ILs and difficulties in addressing the 
conundrum of structure-property relationships(Reddy 2015). Another disadvantage associated when applying ionic liquids with halide anions which bring relatively high viscosities lead to processing difficulties during the dissolution process. However ionic liquids with anions such as acetate, formate, and phosphate possess lower viscosities that facilitate their use for various applications (Isik et al. 2014)

\subsubsection{ILs Applications}

ILs have industrial applications in as widespread areas as pharmaceutics, analytical chemistry, extraction, materials science and as electrolytes in batteries. ILs have shown great versatility in the field of cellulose technology, including dissolution for regeneration purposes (Kosan et al. 2008; Viswanathan et al. 2006), homogeneous derivatization (Gericke et al. 2012), and biomass processing including wood component separation (Froschauer et al. 2013; Sun et al. 2009). Some of them are more detailed as follows:

\subsubsection{Cellulose Based Ion Gels}

1-butyl-3-methylimidazolium chloride (BMIMCl) is one of the most effective ionic liquid solvents for breaking down the strong intra- and inter-molecular interactions; when cellulose is dissolved in BMIMCl an unexpected gel formation could be observed after some days ( Figure 5.7).

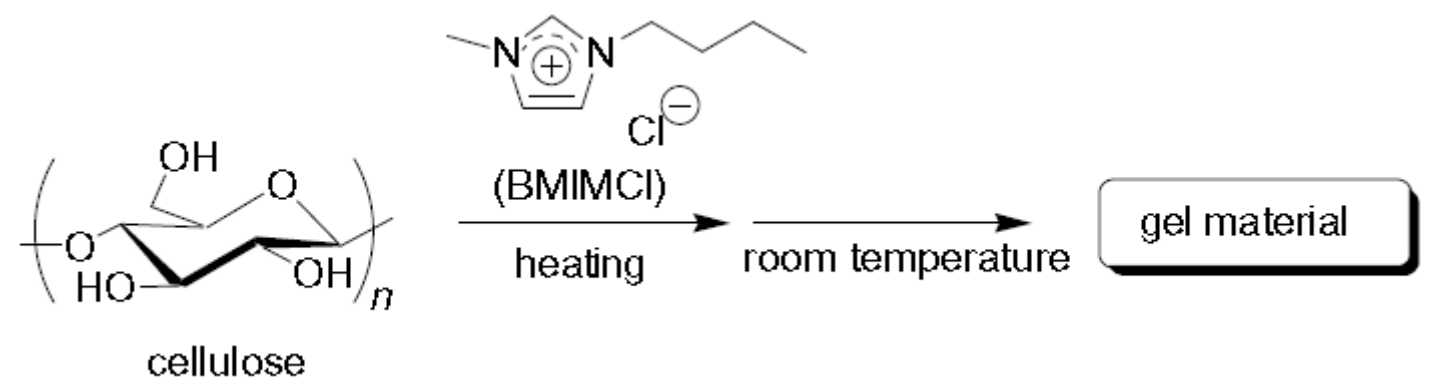

Figure 5.7 Procedure for the preparation of gel material from a solution of cellulose in BMIMCl (Kadokawa et al. 2008).

This has been explained by the development of noncrystalline cellulose aggregates in the solution upon the gradual absorption of water due to strong hygroscopic nature of BMIMCl. When the gel was heated up to $120^{\circ} \mathrm{C}$, it became soft and gradually turned into a fluid at $150^{\circ} \mathrm{C}$. The soft material that is formed at $120^{\circ} \mathrm{C}$ can be transformed into a gel by standing 
it at room temperature for 2 days. The regenerated gel displays better transparency because of more homogeneous distribution of the nano-domains with the heat treatment (Kadokawa et al. 2008).

Novel ion-gels bearing lithium borate were synthesized by Matsumi et al via condensation between cellulose and boric acids in IL to give materials with improved conductivity (Matsumi et al. 2009).Cellulose was first dissolved in the ionic liquid and the condensation reaction was carried out in the solution with the addition of boric acid. The measured conductivities of the ion gels were comparable to the ionic liquid itself, meaning that the immobilization of the ionic liquid in the solid matrix did not induce a significant decrease in the conductivity. A general pathway to prepare cellulose based ion gels is displayed in Figure 5.8.

Ion gels combines the properties of hydrogels (ionic character) and organogels which. have many applications in the fields of electrolytic membranes, lithium-ion batteries, fuel cells, electrochemical- and bio-sensors, actuators and separation membranes (Kawasaki and Iwasa 2012; Néouze et al. 2006).

Zhu et al reviewed the dissolution of cellulose with ionic liquids and its application(Zhu et al. 2006). Based on their review, cellulose can be dissolved, without derivation, in some hydrophilic ionic liquids, such as 1-butyl-3-methylimidazolium chloride (BMIMCl) and 1allyl-3-methylimidazolium chloride (AMIMCl). 


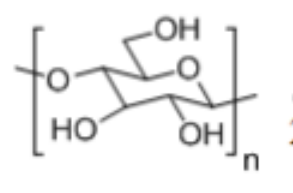

1-Dissolution in ionic liquid

2- Hydration at ambient environment
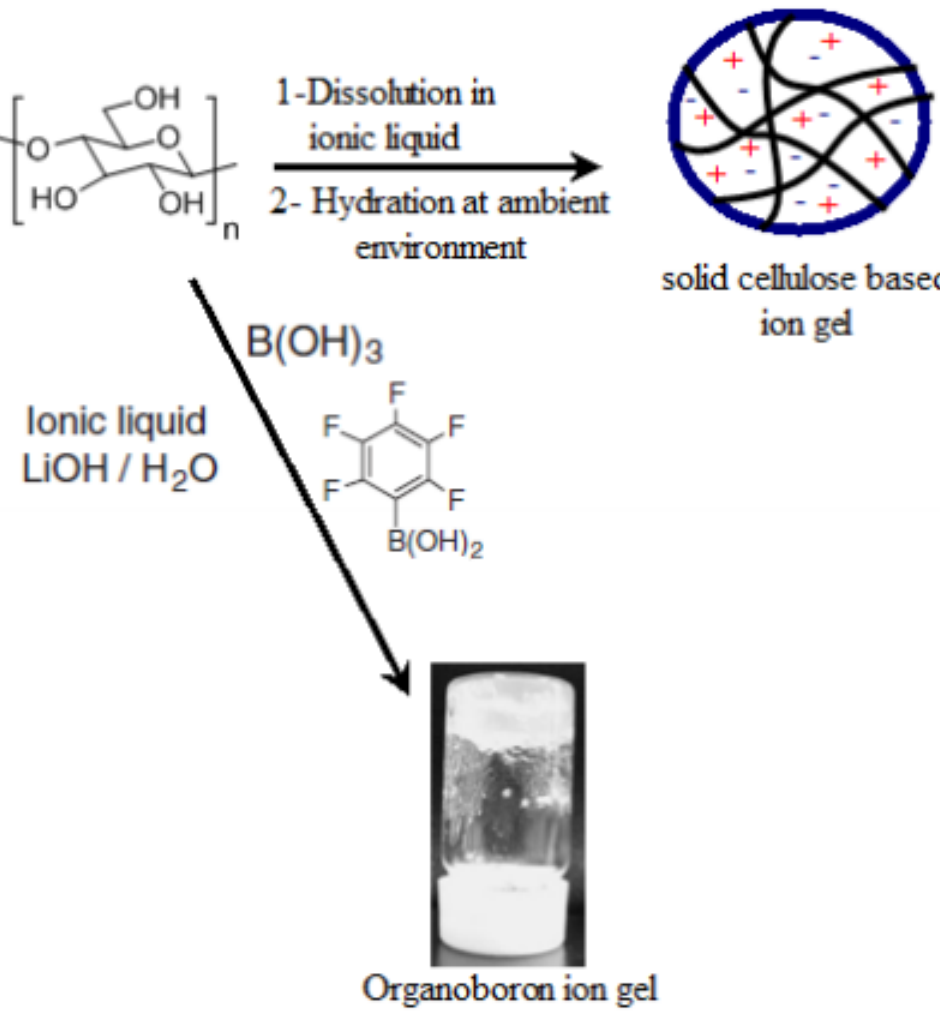

solid cellulose based

ion gel

Figure 5.8 Two different approaches to produce cellulose containing ion gels.

Using microwave heating the dissolution process is significantly accelerated. Cellulose can be easily regenerated from its ionic liquid solutions by addition of water, ethanol or acetone. After its regeneration, the ionic liquids can be recovered and reused (Figure 5.9).

Ethanol, Latic Acid

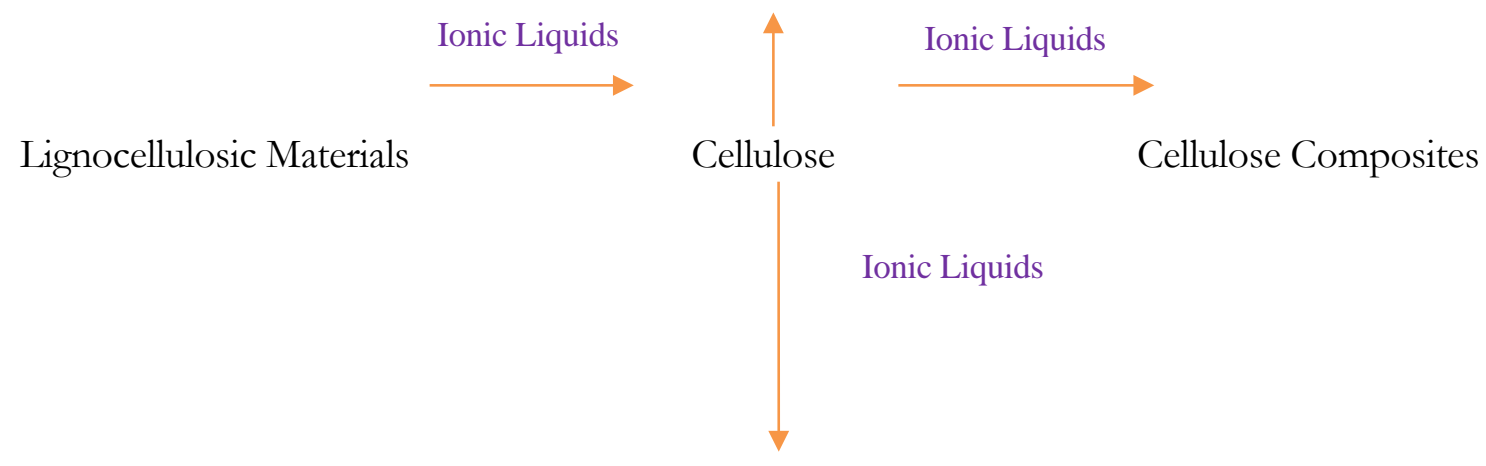

Cellulose Derivatives

Figure 5.9 Dissolution of cellulose with ionic liquids and its application 


\subsubsection{Fraction of lignocellulosic materials}

Based on the fact that cellulose can be dissolved in ionic liquids and it can be easily regenerated from the ionic liquids, Myllymaki and Aksela have found a facile method of fraction of lignocellulosic materials although their objective is delignification from lignocellulosic materials for pulping process (Myllymäki and Aksela 2005).

Lignocellulosic materials, such as wood and straw, are directly dissolved in BMIMCl under microwave irradiation and/or pressure. Then cellulose is precipitated from the BMIMCl solution by the addition of water, and other organic compounds, such as lignin and extractives, still remain in the solution. A similar approach is also used to extract cellulose from lignocellulosic materials. Results have shown that conversion this extracted cellulose into ethanol has a much higher yield than other refined cellulose, such as from lignocellulosic materials pretreated by steam explosion or chemical pretreatment

\subsubsection{Preparation of cellulose derivatives}

Turner et al. reported that the homogeneous acetylation of cellulose could be carried out in AMIMCl without any catalysts and cellulose acetates with a wide range of degree of substitution could be obtained (Turner et al. 2004).

Abbott et al. found the efficient O-acetylation of cellulose could be accomplished using a zinc based ionic liquid(Abbott et al. 2005) .

Acylation and carbanylation of cellulose in BMIMCl without any catalysts under mild conditions, low excess of reagent and a short reaction time has been also carried out(Barthel and Heinze 2006). In all these reports, the reaction media (ionic liquids) could be easily recycled and reused.

\subsubsection{Preparation of cellulose composites}

Preparation of cellulose composites using ionic liquids has broadened the conventional cellulose application scope. The incorporated functional additives can be either dissolved (e.g., dyes, complexants, other polymers) or dispersed (e.g., nano-particles, clays, enzymes) 
in the ionic liquids before or after dissolution of the cellulose. Xie at al. have prepared wool keratin/cellulose composite materials such as fiber and membrane using BMIMCl as a solvent (Xie et al. 2005).

Ionic liquids are ideal to dissolve cellulose; hence they have been extensively employed for the preparation of cellulose-polymer blends.First of all, cellulose and the polymer are dissolved together in the ionic liquid. Afterwards, the resulting homogeneous solution is precipitated to recover the blended polymeric mixture and to remove the ionic liquid. Similarly, polyamines were incorporated into cellulose with a similar preparation method in which cellulose solutions were mixed with different amounts of polyamines to form a surface functionalized cellulose composite (Turner et al. 2005). The prepared cellulose-polyamine composite films and beads provided high loading of primary amines on the surface allowing direct one-step bioconjugation of active species using an ionic liquid (IL) dissolution and regeneration process. Films and bead architectures were prepared and used as immobilization supports for laccase as a model system. The applicability of these materials for use as sensing devices to detect various compounds including polyphenols, aromatic amines, and aminophenols and as solid support materials for enzyme-catalyzed transformations has also been established (Turner et al. 2005)(Figure 5.10).

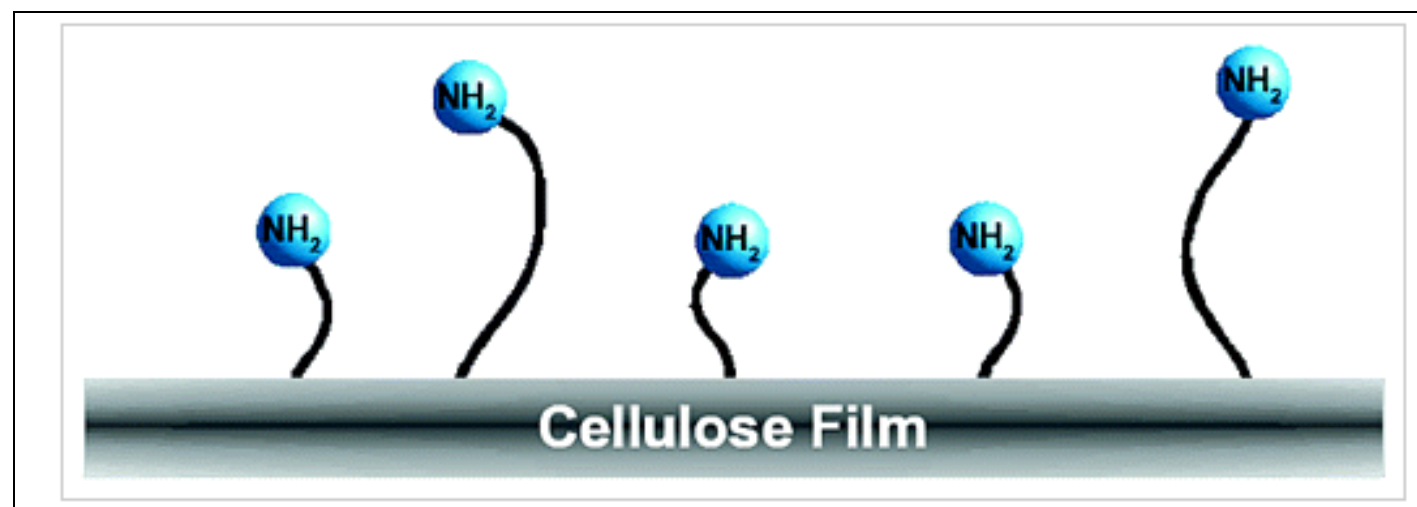

Figure 5.10 Schematic representation of cellulose-polyamine films with protruding primary amines (Turner et al. 2005).

In another study, natural biopolymer wool was blended with cellulose by dissolving both materials in a mutual ionic liquid solvent such as 1-butyl-3-methylimidazolium chloride 
(BMIMCl).The solution containing both materials was precipitated in water giving a composite material with better thermal stability than the individual components.Moreover, the blend films exhibited an increasing trend of tensile strength with increase in cellulose content in the blends which could be used for the development of wool-based materials with improved mechanical properties. It is also worth mentioning that the ionic liquidsolvent was recycled with high yield and purity after composite preparation (Hameed and Guo 2010).

\subsubsection{Polymerized Ionic Liquid-Cellulose Composites}

Aside from the utilization of ionic liquids as solvent, cellulose composites can also be prepared by direct polymerization of ionic liquid monomers in the presence of cellulose as depicted in Figure 5.11. Imidazolium-based ionic liquid monomers are ideal candidates to combine with cellulose due to their high affinity and compatibility (Swatloski et al. 2002). Imidazolium type ionic liquid monomer, 1-(3-acryloyloxybutyl)-3-methylimidazolium bromide (AcMIMBr) was prepared by Murakami et al. A cellulose-polymerized ionic liquid composite was prepared by in-situ polymerization method using two kinds of ionic liquids, which dissolved cellulose as a solvent and had a polymerizable acrylate group, respectively. The results of the IR spectrum and elemental analysis indicated that the isolated product was a composite consisting of cellulose and the polymerized ionic liquid. Thermal findings of the composite material indicate that the cellulose was properly compatibilized with the polymerized ionic liquid system (Murakami et al. 2007). 

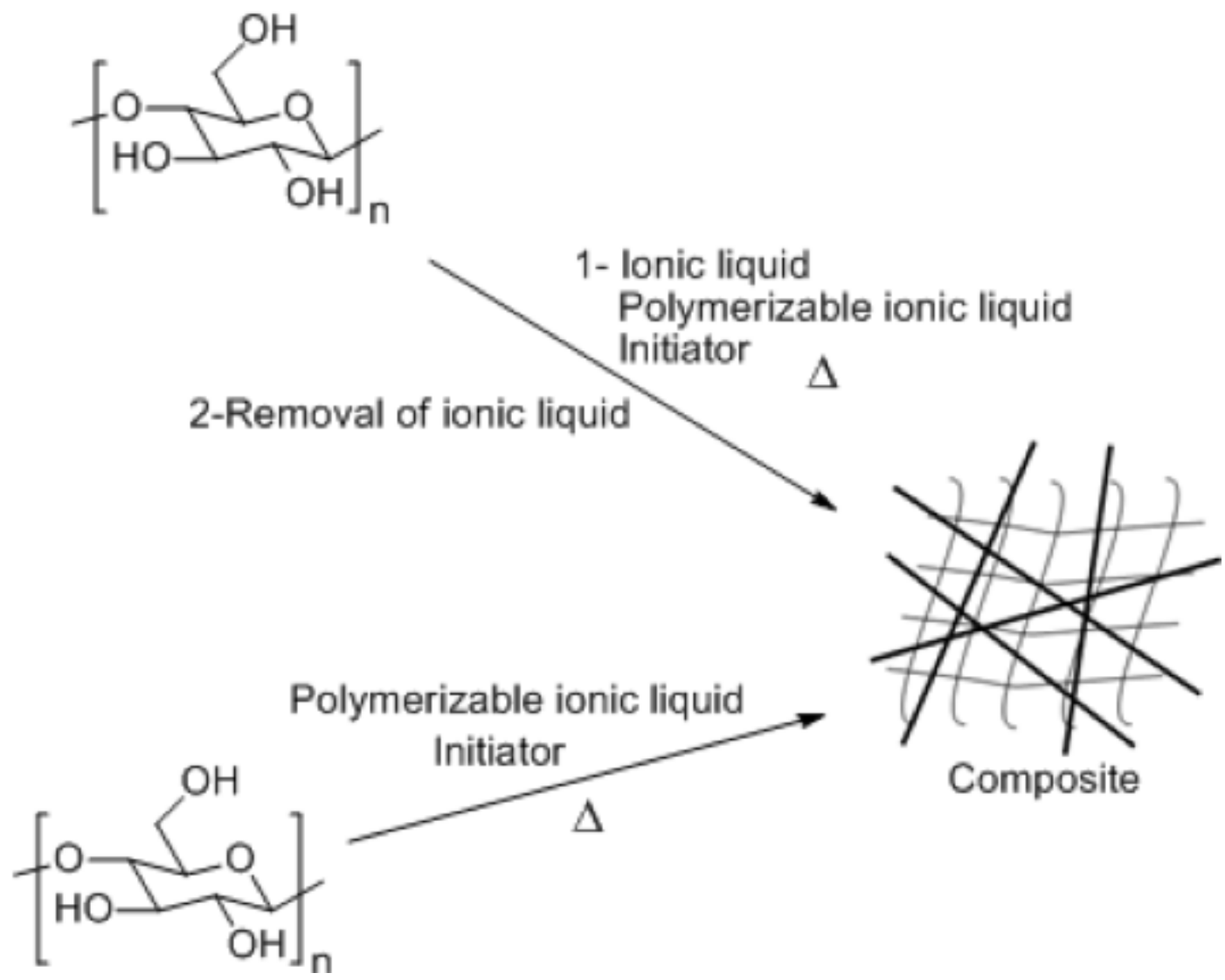

Figure 5.11 Two main approaches to obtain cellulose-poly (ionic liquid) composites. $\Delta=$ heat given to the system (Isik et al. 2014).

Prasad et al.2010 obtained a porous cellulose polymeric ionic liquid based material using templating technique with oil/ionic liquid emulsion system. In the first step, the cellulose was dissolved in BMIMCl and AcMIMBr, 1-(3-acryloyloxypropyl)-3-vinylimidazolium bromide (AcVIMBr) monomer mixture (Figure 5.12).After, the mixture was in-situ polymerized at elevated temperature. Then, the synthesized system was mixed with corn oil and sorbitan monooleate prior to the sonication process. The sonicated sample was treated with methanol, acetone, and hexane solvent system to produce the porous composite material. The composite displayed nonhomogeneous pore size distribution, pore sizes being in the range of $0.15-1.3 \mu \mathrm{m}$ together with smaller sizes of 30-70 nm. 


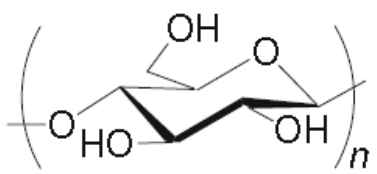

Cellulose

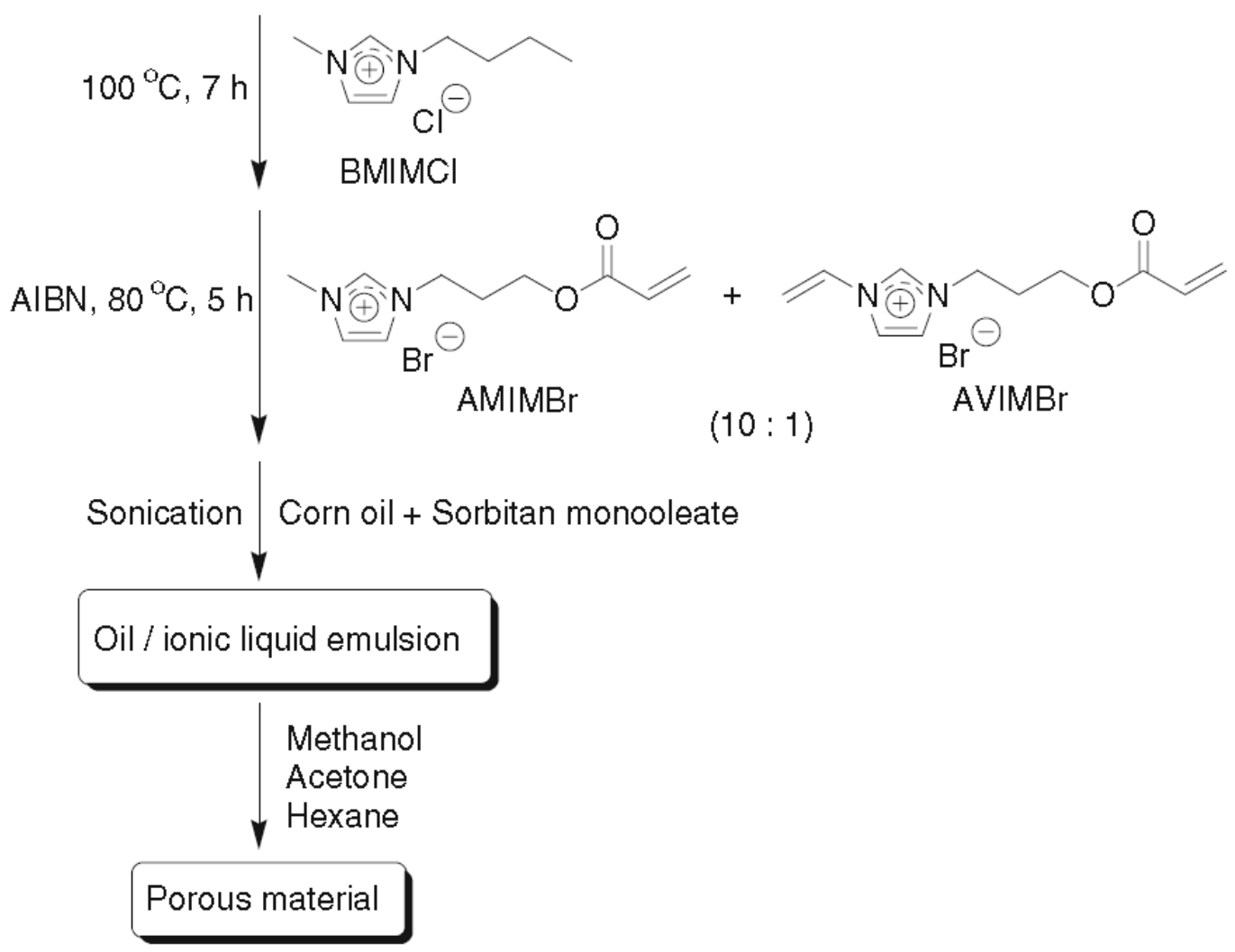

Figure 5.12 Preparation procedure of cellulose-based ionic porous material compatibilized with polymeric ionic liquid.

Recently a facile in situ polymerization method to produce cellulose-polymerized ionic liquid composite coatings has been developed. In this work, cellulose, treated with a quaternary ammonium ionic liquid monomer at room temperature, was applied onto a surface and photopolymerized in the presence of a photoinitiator. The resulting coating with 5 wt \% of cellulose displayed good transparency indicating a good compatibility between the ionic liquid monomer and cellulose for preparation of poly(ionic liquid)/cellulose composite (Isik et al. 2013). 


\subsubsection{All-cellulose composites}

Fully bio-based and biodegradable all-cellulose composites were prepared in the form of films by partial dissolution of two cellulose sources: a commercially available microfibrillated cellulose (MFC) and filter paper (FP). The solvent selected for this work was the ionic liquid 1-butyl-3-methylimidazolium chloride ([C4mim] Cl). Both cellulose sources were partially dissolved at $80 \mathrm{C}$ and consolidated by partial dissolution, resulting in excellent mechanical properties.

The cellulose I crystalline structure was retained after the partial dissolution, and high mechanical properties were achieved. They also showed that penetration of the ionic liquid was limited to the surface, forming a skin-core structure (Duchemin et al. 2009).

\subsubsection{Other ILs applications}

Mathew et al. used ionic liquids to partially dissolve the cellulose nanofiber network for ligament applications(Mathew et al. 2012). The partially dissolved networks were regenerated in water and were cleaned by extensive washing with distilled water and consolidation at $60 \mathrm{C}$. Ligament-type prototypes were prepared by rolling the nanopaper sheets into tubules after the surface selective dissolution and regeneration in water. Figure 5.13 shows the ligament proto-types prepared and the microstructure with nanofibers embedded in the cellulose matrix.
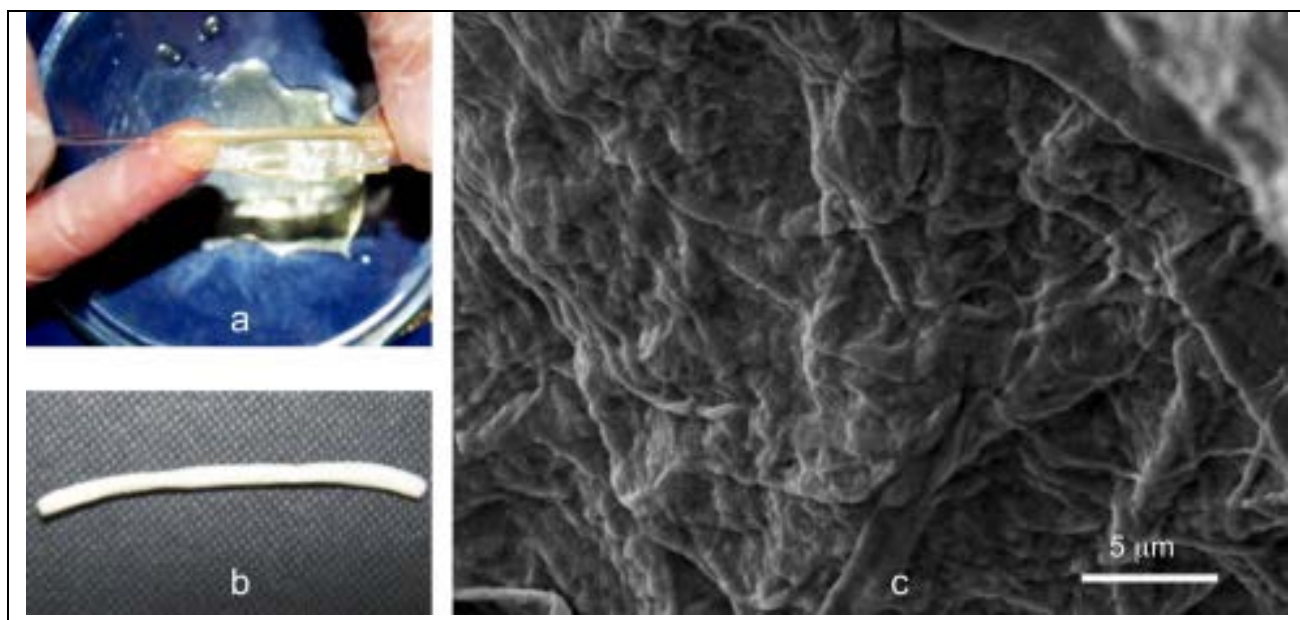

Figure 5.13(a) ligament prototype processing using ionic liquid dissolution, (b) the prototype in tubule form and (c) the microstructure of the prototype walls after partial dissolution (Oksman et al. 2016). 
Fibrous cellulose nanocomposites scaffolds were developed and evaluated for their potential as ligament or tendon substitute. The nanocomposites were prepared by partial dissolution of cellulose nanofiber networks using ionic liquid at $80^{\circ} \mathrm{C}$ for different time intervals.

Mashkour et al. report the production and properties of a strong anisotropic superparamagnetic cellulose nanocomposite (ASPCNC) film with high in-plane anisotropy prepared using ionic liquid (IL)-based peeling and in situ welding processes.The results show highly significant relationships between increasing partial dissolution time as the development index of the IL-based peeling and in situ welding procedures and improvements in the mechanical properties and structural, mechanical, and magnetic in-plane anisotropy of the ASPCNCs .The process resulted in flexible, anisotropic and super magnetic nanocomposite films that are expected to have advanced applications as storage devices, magnetic micro-robots and micro switches.(Mashkour et al. 2014).

\subsubsection{Fiber Spinning}

Nanocellulose (NC) represents a new class of cellulose-based material that may have potential in continuous fibers. It can be extracted from trees, plants, some marine creatures such as tunicates, and certain bacteria or algae (Moon et al. 2011). NC is not a single material type but a family of materials with very different characteristics, largely due to differences in preparation methodology and source. Here, we focus on plant-derived nanocelluloses. These nanocelluloses are projected to be less expensive than many other nanomaterials and have the potential to be produced in large volumes (Hansen et al. 2014). They also have an impressive strength-to-weight ratio and have so far shown few environmental, health, and safety concerns in their unmodified state (Hansen et al. 2014).

Spinning of continuous fibers from NC is an intriguing option because it has the potential to effectively use the one-dimensional nature and very fine dimensions of NC while maintaining its Cellulose I structure.

Unlike the cellulose-based fibers previously mentioned (natural fibers, regenerated cellulose), NC can be made into continuous fibers of submicron diameter. This reduction in fiber diameter results in continuous nanofibers with intriguing characteristics such as very 
large surface area, transparency, and fine, controllable porosity when made into mats (Giesa and Buehler 2013). Because of these characteristics, nanofibers are being considered in applications such as filters, sensors, optical electronics, catalysts, and tissue scaffolding (Medeiros et al. 2009).

However, efficient and cost-effective preparation of continuous NC fibers is challenging and a variety of approaches have been investigated that either spin fibers from 100\% NC dispersions or from NC combined with polymers. To date most of the research on continuous NC fibers has focused on monofilaments or electrospun fibrous mats, either directly spinning fiber from NC dispersions or combining NC with a polymer. Wet-, dry-, melt- and electrospinning methods have been used as well as specialty processes (e.g., microfluidics) to produce continuous fibers. The various approaches used with NC are summarized schematically in Figure 5.14 and mostly involves solution spinning methods (e.g., wet spinning, dry spinning), melt spinning, and electrospinning.

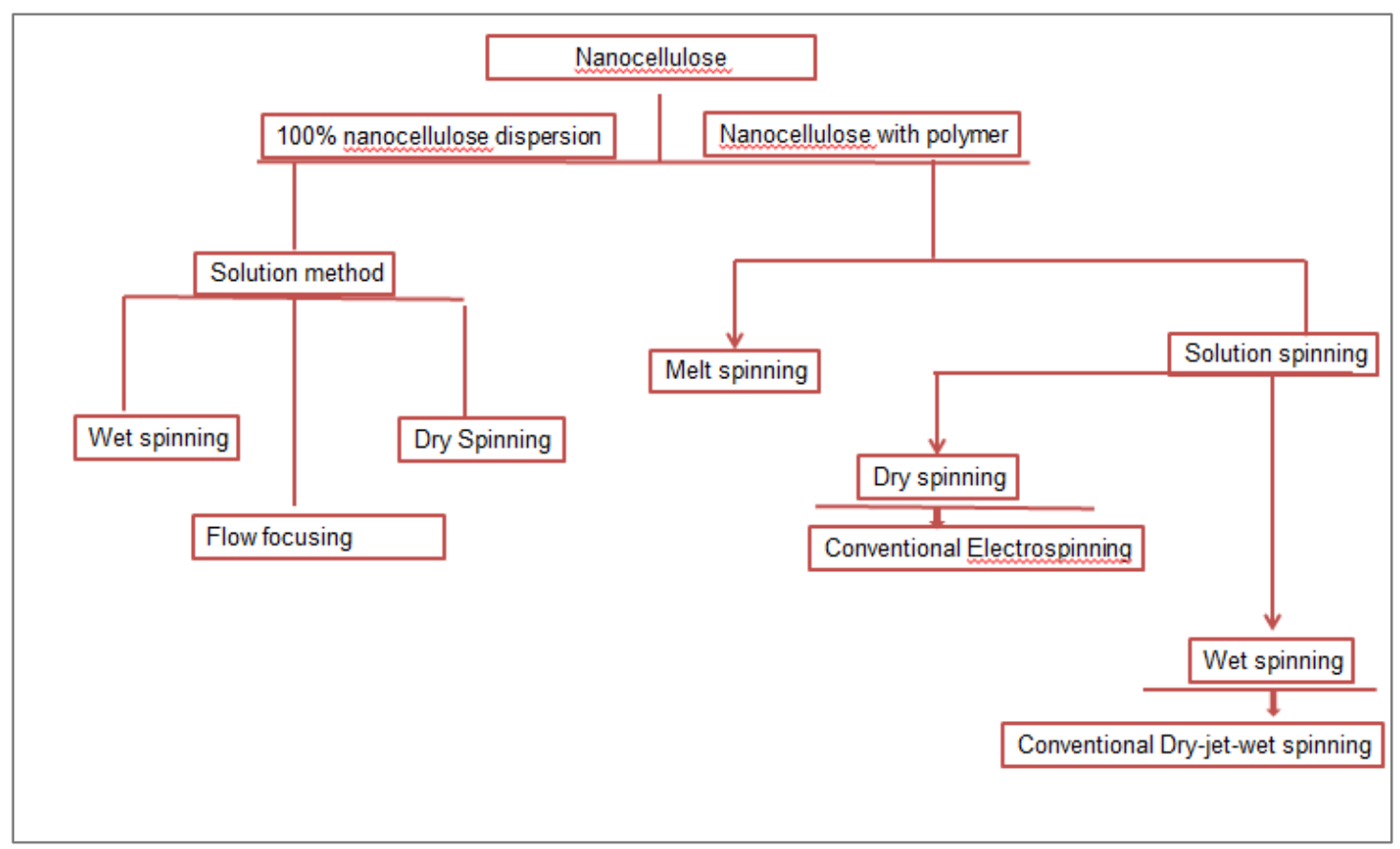

Figure 5.14 various approach for preparing continuous fibers from nanocellulose for different applications. 
Melt spinning is the most common method for commercial production of polymer fibers and is often the most economical. Not surprisingly, it is used in spinning most commodity plastics. In melt spinning, molten plastic is pumped under high pressure through the spinneret. In solution spinning methods (e.g., wet spinning or dry spinning), a viscous solution of polymer (sometimes called a spinning dope) is used rather than a melt.

In melt spinning, molten plastic is pumped under high pressure through the spinneret. In solution spinning methods (e.g., wet spinning or dry spinning), a viscous solution of polymer (sometimes called a spinning dope) is used rather than a melt. While the solution spinning methods tend to be more expensive than melt spinning methods, they often result in greater mechanical performance by carefully controlling the level of molecular entanglement, which can result in more highly drawn fibers. In dry spinning, the dope is forced through the spinneret and solvent is removed by evaporation, often in a hot atmosphere. In wet spinning, the material leaving the spinneret is submerged in a liquid where the solvent is either removed through diffusion or through precipitation by chemical reaction (Carley 1993). The fiber is then dried to remove remaining liquid.

In electrospinning, a large electric field is applied between the spinneret and a conductive collector, resulting in uniaxial elongation of a jet of polymer melt, emulsion or (more often) a solution or dispersion (Persano et al. 2013).

Preparation of NC results in aqueous dispersions. These dispersions, particularly those of CNFs, are somewhat analogous to the polymer solutions (spinning dopes) used to spin fibers. Rather than long polymer molecules dissolved in a solvent, CNFs are dispersed in water. Therefore, it is perhaps not surprising that some research to date has centered on directly spinning these NC dispersions.Researchers also have noted that wet-spinning is a promising alternative technique for fast and efficient dehydration of CNF dispersions.Rather than CNCs, CNFs have exclusively been used to spin 100\% NC dispersions because of the high aspect ratio and fibril entanglement/interaction that is necessary. 
Walther et al. also wet spun 100\% TEMPO CNFs and demonstrated how the fibers could be functionalized for hydrophobicity, conductivity, and magnetism. These early fibers showed evidence of some porosity (Walther et al. 2011).

While some work has been performed on melt spinning NC-polymer blends, most investigations have centered on solution spinning methods. Solution spinning can avoid challenges such as the low thermal stability of NC and difficulties in dispersing dry NC. Additionally, techniques such as electrospinning can be used to prepare fibers of nanoscale diameter, and fiber mats with beneficial characteristics such as high surface area and a fine, porous structure. Such nanofibers are of great interest and can potentially enable nanocellulose use in new application areas such as filtration, catalysis, and tissue scaffolding (Medeiros et al. 2009).However, spinning with solutions has its own challenges. The effect of CNFs is especially large due to its entangled network structure, which leads to high viscosity and formation of gels even at low concentrations. As a result, less CNFs are usually added to spinning dopes and more liquid needs to be removed than if CNCs are used (Xu et al. 2014).

\subsubsection{Electrospinning application}

Aside from being a conceptually simple and versatile technique, electrospinning offers several other potential advantages from a fiber preparation standpoint with regard to NC (Teo and Ramakrishna 2006). The large electrostatic fields and the shear forces in the liquid jet during electrospinning help induce alignment of both polymer chains and NC. CNCs have almost exclusively been used as the NC component due, in part, to the fact that the large viscosity increases with CNF addition make electrospinning more difficult and tend to result in larger diameter fibers.A variety of polymers have been investigated as matrices. For example, CNCs have been added to clear polymers such as polystyrene (Rojas et al. 2009) and poly(methyl methacrylate) (Dong et al. 2012a) to try and improve performance in applications where transparency is important. Use of biobased polymers, such as poly(lactic acid) (Liu et al. 2012) and cellulose acetate (Herrera et al. 2011; Vallejos et al. 2012), results in $100 \%$ biobased composites. 
In other studies, adding $\mathrm{NC}$ to biocompatible polymers (e.g., poly( $\varepsilon$-caprolactone) (Zoppe et al. 2009), poly(acrylic acid) (Lu and Hsieh 2009), poly(ethylene oxide) (Motaung and Mokhena 2015; Park et al. 2007), poly(vinyl alcohol) (Medeiros et al. 2008; Peresin et al. 2010b) positively impact spinning and performance of electrospun mats for biomedical applications (e.g., tissue engineering).

Others have shown how stable CNC-inorganic particle dispersions could be electrospun with polyvinyl alcohol into magnetically active mats that could potentially be used in separation applications (Nypelö et al. 2014).

Much of the research has centered on the effects of CNC on the improvement of mechanical properties, particularly in biocompatible and/or water-soluble polymer matrices. This may prove useful for biomedical applications where improvements in matrix properties are often needed and the economic feasibility of electrospun mats is considered more possible. In this contextLee and Deng showed that adding 15\% CNCs to a highly hydrolyzed polyvinyl alcohol doubled the tensile modulus and increased the strength by $86 \%$ in random electrospun mats (Lee and Deng 2012) .

Electrospinning of cellulose from conventional solvent systems has been complicated because of their toxicity and harsh conditions required for dissolution.Hence, solubility of cellulose in ionic liquids facilitated the electrospinning process of this material to obtain fibers with tunable properties(Isik et al. 2014)

$\mathrm{Xu}$ et al. used a 1-allyl-3-methylimidazolium chloride (AMIMCl) and dimethylsulfoxide (DMSO) solvent system in order to produce electrospun cellulose fibers. The fibers were collected onto a rotating copper-wire drum under a high humidity and the remaining ionic liquid was eliminated resulting in a self-standing solid electrospun network (see Figure 5.15). XRD analysis revealed that the cellulose fibers were almost entirely amorphous after the electrospinning process (Xu et al. 2008). 

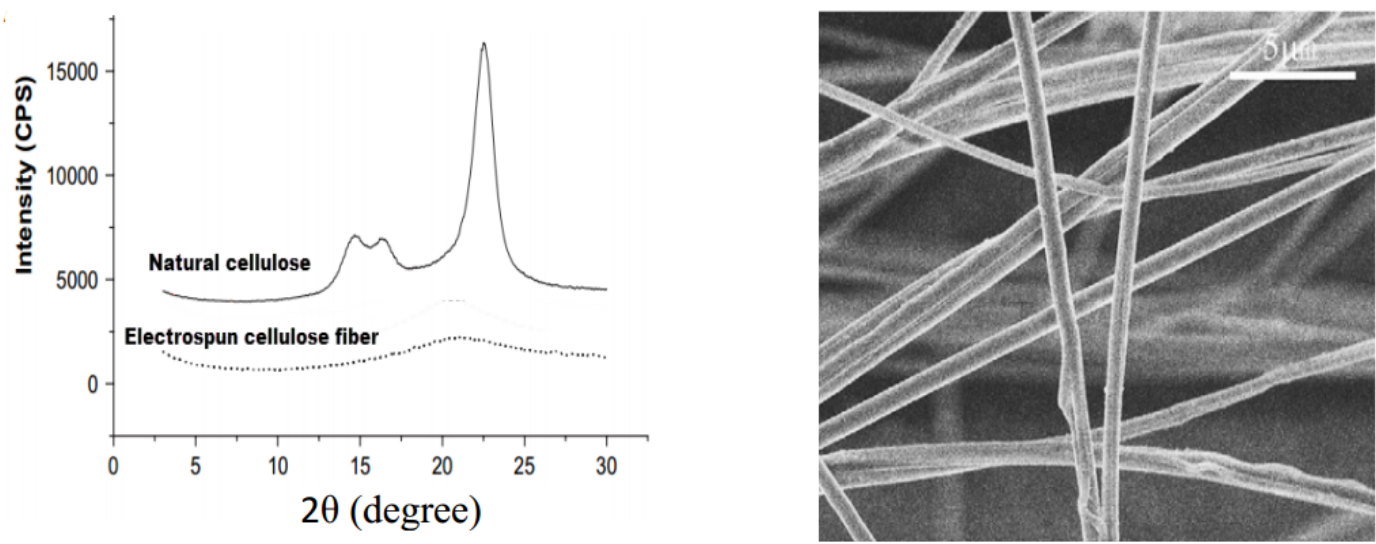

Figure 5.15 (Left) XRD (X-ray diffraction) patterns of cellulose and electrospun fibers from AMIMCl; (Right) SEM (scanning electron microscope) image of fibers produced from 5 wt \% solution; reprinted from (Xu et al. 2008).

Another study was performed by Ahn et al. to investigate the effect of cosolvents on both electrospinnability and the resulting fiber morphology.1-ethyl-3-methylimidazolium acetate was used as the solvent to dissolve cellulose and dimethylformamide (DMF) and dimethylacetamide (DMAc) were utilized as cosolvents.It was shown that the spinnability of cellulose increased as the concentration of cosolvent increases in the solution.Regardless of the cosolvent type, finer fibers were obtained with higher cosolvent concentrations, resulting in better web uniformity, thermal stability and crystallinity(Ahn et al. 2012).

The reason behind the improvement of electrospinnability of cellulose with the addition of cosolvent is that the cosolvent penetrates into the gaps between the chains and the ionic liquid enhancing the chain mobility due to weaker interaction with the chains. As a result, cellulose chains can be easily elongated and fibrillated during the process(Isik et al. 2014).

Besides using only cellulose, other polymeric materials can also be blended prior to the electrospinning process to create hybrid fibers with new properties. For instance, Viswanathan et al. prepared cellulose-heparin composites using ionic liquids. On one hand cellulose was dissolved in BMIMCl and on the other hand heparin was dissolved in 1-ethyl3-methylimidazolium benzoate. Prior to the electrospinning the two solutions were mixed and the blended fibers were collected in ethanol through precipitation of electrospun fibers. 
They found that the composite fibers displayed anticoagulant activity indicating that bioactivity of heparin remained unaffected. These composite fiber materials can be used for the construction of artificial blood vessels with excellent blood compatibility (Viswanathan et al. 2006).

\subsection{Summary}

Ionic liquids are suitable solvents for cellulose dissolution and for fibre spinning. The reactions are often quicker and easier to carry out than in conventional organic solvents. The use of ionic liquids for cellulose dissolution comes from their unique properties to interact with the strong hydrogen bonds of cellulose. This phenomenon is efficient and induces substantial changes in the surface properties of NFC which can be translated into new processing technologies, cellulose functionalization methods and new cellulose materials including composites, fibers and ion gels.

\subsection{References}

Abbott AP, Bell TJ, Handa S, Stoddart B (2005) O-Acetylation of cellulose and monosaccharides using a zinc based ionic liquid Green chemistry 7:705-707

Ahn Y, Hu D-H, Hong JH, Lee SH, Kim HJ, Kim H (2012) Effect of co-solvent on the spinnability and properties of electrospun cellulose nanofiber Carbohydrate Polymers 89:340-345

Barthel S, Heinze T (2006) Acylation and carbanilation of cellulose in ionic liquids Green chemistry 8:301-306

Blake DM, Moens L, Rudnicki D, Pilath H (2006) Lifetime of imidazolium salts at elevated temperatures Journal of Solar Energy Engineering 128:54-57

Carley JF (1993) Whittington's dictionary of plastics. CRC Press,

Chiappe C, Pieraccini D (2005) Ionic liquids: solvent properties and organic reactivity Journal of Physical Organic Chemistry 18:275-297

Croitoru C, Patachia S (2016) Long-Chain Alkylimidazolium Ionic Liquid Functionalization of Cellulose Nanofibers and Their Embedding in HDPE Matrix International Journal of Polymer Science 2016

Dong H, Strawhecker KE, Snyder JF, Orlicki JA, Reiner RS, Rudie AW (2012) Cellulose nanocrystals as a reinforcing material for electrospun poly (methyl methacrylate) fibers: Formation, properties and nanomechanical characterization Carbohydrate Polymers 87:2488-2495 
Duchemin BJ, Mathew AP, Oksman K (2009) All-cellulose composites by partial dissolution in the ionic liquid 1-butyl-3-methylimidazolium chloride Composites Part A: Applied Science and Manufacturing 40:2031-2037

Earle MJ, Seddon KR (2000) Ionic liquids. Green solvents for the future Pure and Applied Chemistry 72:1391-1398

Frone AN, Panaitescu DM, Donescu D (2011) Some aspects concerning the isolation of cellulose micro-and nano-fibers UPB Buletin Stiintific, Series B: Chemistry and Materials Science 73:133-152

Froschauer C, Hummel M, Iakovlev M, Roselli A, Schottenberger H, Sixta H (2013) Separation of hemicellulose and cellulose from wood pulp by means of ionic liquid/cosolvent systems Biomacromolecules 14:1741-1750

Gericke M, Fardim P, Heinze T (2012) Ionic liquids—promising but challenging solvents for homogeneous derivatization of cellulose Molecules 17:7458-7502

Giesa T, Buehler MJ (2013) Nanoconfinement and the Strength of Biopolymers Annual review of biophysics 42:651-673

Graenacher C (1934) Cellulose solution. US Patent 1943176.

Hameed N, Guo Q (2010) Blend films of natural wool and cellulose prepared from an ionic liquid Cellulose 17:803-813

Hansen F, Brun V, Keller E, Wegner T, Meador M, Friedersdorf L (2014) Cellulose nanomaterials-a path towards commercialization workshop report

Herrera N, Mathew AP, Wang L, Oksman K (2011) Randomly oriented and aligned cellulose fibres reinforced with cellulose nanowhiskers, prepared by electrospinning Plastics, Rubber and Composites 40:57-64

Hummel M et al. (2015) Ionic Liquids for the Production of Man-Made Cellulosic Fibers: Opportunities and Challenges. In: Cellulose Chemistry and Properties: Fibers, Nanocelluloses and Advanced Materials. Springer, pp 133-168

Isik M, Gracia R, Kollnus LC, Tomé LC, Marrucho IM, Mecerreyes D (2013) Cholinium-based poly (ionic liquid) s: synthesis, characterization, and application as biocompatible ion gels and cellulose coatings ACS Macro Letters 2:975-979

Isik M, Sardon H, Mecerreyes D (2014) Ionic liquids and cellulose: Dissolution, chemical modification and preparation of new cellulosic materials International Journal of Molecular Sciences 15:11922-11940

Kadokawa J-i, Murakami M-a, Kaneko Y (2008) A facile preparation of gel materials from a solution of cellulose in ionic liquid Carbohydrate Research 343:769-772

Kawasaki M, Iwasa Y (2012) Electronics:'Cut and stick'ion gels Nature 489:510-511

Kokol V, Božič M, Vogrinčič R, Mathew AP (2015) Characterisation and properties of homo-and heterogenously phosphorylated nanocellulose Carbohydrate Polymers 125:301-313

Kosan B, Michels C, Meister F (2008) Dissolution and forming of cellulose with ionic liquids Cellulose 15:59-66 
Lan W, Liu C-F, Yue F-X, Sun R-C, Kennedy JF (2011) Ultrasound-assisted dissolution of cellulose in ionic liquid Carbohydrate Polymers 86:672-677

Lee H, Hamid S, Zain S (2014) Conversion of lignocellulosic biomass to nanocellulose: structure and chemical process The Scientific World Journal 2014

Lee J, Deng Y (2012) Increased mechanical properties of aligned and isotropic electrospun PVA nanofiber webs by cellulose nanowhisker reinforcement Macromolecular Research 20:76-83

Li J et al. (2012) Homogeneous isolation of nanocellulose from sugarcane bagasse by high pressure homogenization Carbohydrate Polymers 90:1609-1613

Liebert T, Seifert M, Heinze T (2008) Efficient Method for the Preparation of Pure, Water-Soluble Cellodextrines. In: Macromolecular symposia, 2008. Wiley Online Library, pp 140-149

Liu D, Yuan X, Bhattacharyya D (2012) The effects of cellulose nanowhiskers on electrospun poly (lactic acid) nanofibres Journal of Materials Science 47:31593165

Lu P, Hsieh Y-L (2009) Cellulose nanocrystal-filled poly (acrylic acid) nanocomposite fibrous membranes Nanotechnology 20:415604

Mashkour M, Tajvidi M, Kimura F, Yousefi H, Kimura T (2014) Strong highly anisotropic magnetocellulose nanocomposite films made by chemical peeling and in situ welding at the interface using an ionic liquid ACS applied materials \& interfaces 6:8165-8172

Mathew AP, Oksman K, Pierron D, Harmand M-F (2012) Fibrous cellulose nanocomposite scaffolds prepared by partial dissolution for potential use as ligament or tendon substitutes Carbohydrate Polymers 87:2291-2298

Matsumi N, Nakamura Y, Aoi K, Watanabe T, Mizumo T, Ohno H (2009) Enhanced ionic conduction in organoboron ion gels facilely designed via condensation of cellulose with boric acids in ionic liquids Polymer Journal 41:437

Medeiros ES, Glenn GM, Klamczynski AP, Orts WJ, Mattoso LH (2009) Solution blow spinning: A new method to produce micro-and nanofibers from polymer solutions Journal of Applied Polymer Science 113:2322-2330

Medeiros ES et al. (2008) Electrospun nanofibers of poly (vinyl alcohol) reinforced with cellulose nanofibrils Journal of Biobased Materials and Bioenergy 2:231-242

Missoum K, Belgacem MN, Barnes J-P, Brochier-Salon M-C, Bras J (2012) Nanofibrillated cellulose surface grafting in ionic liquid Soft Matter 8:8338-8349

Missoum K, Belgacem MN, Bras J (2013) Nanofibrillated cellulose surface modification: a review Materials 6:1745-1766

Moon RJ, Martini A, Nairn J, Simonsen J, Youngblood J (2011) Cellulose nanomaterials review: structure, properties and nanocomposites Chemical Society Reviews 40:3941-3994 
Motaung TE, Mokhena TC (2015) Effects of mechanical fibrillation on cellulose reinforced poly (ethylene oxide) Mater Sci Appl 6:713-723

Murakami M-a, Kaneko Y, Kadokawa J-i (2007) Preparation of cellulose-polymerized ionic liquid composite by in-situ polymerization of polymerizable ionic liquid in cellulose-dissolving solution Carbohydrate Polymers 69:378-381

Myllymäki V, Aksela R (2005) Dissolution and delignification of lignocellulosic materials with ionic liquid solvent under microwave irradiation WO Pat 17001

Néouze M-A, Le Bideau J, Gaveau P, Bellayer S, Vioux A (2006) Ionogels, new materials arising from the confinement of ionic liquids within silica-derived networks Chemistry of Materials 18:3931-3936

Nypelö T, Rodriguez-Abreu C, Rivas J, Dickey MD, Rojas OJ (2014) Magnetoresponsive hybrid materials based on cellulose nanocrystals Cellulose 21:25572566

Oksman K et al. (2016) Review of the recent developments in cellulose nanocomposite processing Composites Part A: Applied Science and Manufacturing 83:2-18

Olsson C (2014) Cellulose processing in ionic liquid based solvents. Chalmers University of Technology,

Olsson C, Hedlund A, Idström A, Westman G (2014) Effect of methylimidazole on cellulose/ionic liquid solutions and regenerated material therefrom Journal of Materials Science 49:3423-3433

Park WI, Kang M, Kim HS, Jin HJ Electrospinning of poly (ethylene oxide) with bacterial cellulose whiskers. In: Macromolecular symposia, 2007. Wiley Online Library, pp 289-294

Peresin MS, Habibi Y, Zoppe JO, Pawlak JJ, Rojas OJ (2010) Nanofiber composites of polyvinyl alcohol and cellulose nanocrystals: manufacture and characterization Biomacromolecules 11:674-681

Persano L, Camposeo A, Tekmen C, Pisignano D (2013) Industrial upscaling of electrospinning and applications of polymer nanofibers: a review Macromolecular Materials and Engineering 298:504-520

Prasad K, Mine S, Kaneko Y, Kadokawa J-i (2010) Preparation of cellulose-based ionic porous material compatibilized with polymeric ionic liquid Polymer bulletin 64:341-349

Rabideau BD, Agarwal A, Ismail AE (2014) The role of the cation in the solvation of cellulose by imidazolium-based ionic liquids The Journal of Physical Chemistry B 118:1621-1629

Reddy P (2015) A critical review of ionic liquids for the pretreatment of lignocellulosic biomass South African Journal of Science 111:1-9

Rojas OJ, Montero GA, Habibi Y (2009) Electrospun nanocomposites from polystyrene loaded with cellulose nanowhiskers Journal of Applied Polymer Science 113:927935 
Sun N, Rahman M, Qin Y, Maxim ML, Rodríguez H, Rogers RD (2009) Complete dissolution and partial delignification of wood in the ionic liquid 1-ethyl-3methylimidazolium acetate Green chemistry 11:646-655

Swatloski RP, Spear SK, Holbrey JD, Rogers RD (2002) Dissolution of cellose with ionic liquids Journal of the American Chemical Society 124:4974-4975

Tan XY (2016) Catalytic conversion of cellulose into nanocellulose in ionic liquid/Tan Xiao Yun. University of Malaya

Tang L-r, Huang B, Ou W, Chen X-r, Chen Y-d (2011) Manufacture of cellulose nanocrystals by cation exchange resin-catalyzed hydrolysis of cellulose Bioresource Technology 102:10973-10977

Teo WE, Ramakrishna S (2006) A review on electrospinning design and nanofibre assemblies Nanotechnology 17:R89

Thunberg J (2015) Chemical Modification of Electrospun Cellulose Nanofibers. Chalmers University of Technology,

Tian D, Han Y, Lu C, Zhang X, Yuan G (2014) Acidic ionic liquid as "quasihomogeneous" catalyst for controllable synthesis of cellulose acetate Carbohydrate Polymers 113:83-90

Turner MB, Spear SK, Holbrey JD, Daly DT, Rogers RD (2005) Ionic liquidreconstituted cellulose composites as solid support matrices for biocatalyst immobilization Biomacromolecules 6:2497-2502

Turner MB, Spear SK, Holbrey JD, Rogers RD (2004) Production of bioactive cellulose films reconstituted from ionic liquids Biomacromolecules 5:1379-1384

Vallejos ME, Peresin MS, Rojas OJ (2012) All-cellulose composite fibers obtained by electrospinning dispersions of cellulose acetate and cellulose nanocrystals Journal of Polymers and the Environment 20:1075-1083

Viswanathan G, Murugesan S, Pushparaj V, Nalamasu O, Ajayan PM, Linhardt RJ (2006) Preparation of biopolymer fibers by electrospinning from room temperature ionic liquids Biomacromolecules 7:415-418

Vitz J, Erdmenger T, Haensch C, Schubert US (2009) Extended dissolution studies of cellulose in imidazolium based ionic liquids Green chemistry 11:417-424

Walther A, Timonen JV, Díez I, Laukkanen A, Ikkala O (2011) Multifunctional highperformance biofibers based on wet-extrusion of renewable native cellulose nanofibrils Advanced Materials 23:2924-2928

Xie H, Li S, Zhang S (2005) Ionic liquids as novel solvents for the dissolution and blending of wool keratin fibers Green chemistry 7:606-608

Xu A, Wang J, Wang H (2010) Effects of anionic structure and lithium salts addition on the dissolution of cellulose in 1-butyl-3-methylimidazolium-based ionic liquid solvent systems Green chemistry 12:268-275 
Xu A, Zhang Y, Zhao Y, Wang J (2013) Cellulose dissolution at ambient temperature: role of preferential solvation of cations of ionic liquids by a cosolvent Carbohydrate Polymers 92:540-544

Xu S, Zhang J, He A, Li J, Zhang H, Han CC (2008) Electrospinning of native cellulose from nonvolatile solvent system Polymer 49:2911-2917

Xu X, Wang H, Jiang L, Wang X, Payne SA, Zhu J, Li R (2014) Comparison between cellulose nanocrystal and cellulose nanofibril reinforced poly (ethylene oxide) nanofibers and their novel shish-kebab-like crystalline structures Macromolecules 47:3409-3416

Youngs TG et al. (2011) Neutron diffraction, NMR and molecular dynamics study of glucose dissolved in the ionic liquid 1-ethyl-3-methylimidazolium acetate Chemical Science 2:1594-1605

Zhang S, Sun N, He X, Lu X, Zhang X (2006) Physical properties of ionic liquids: database and evaluation Journal of physical and chemical reference data 35:14751517

Zheng W et al. (2011) Effect of cation symmetry on the morphology and physicochemical properties of imidazolium ionic liquids The Journal of Physical Chemistry B 115:6572-6584

Zhu S et al. (2006) Dissolution of cellulose with ionic liquids and its application: a minireview Green chemistry 8:325-327

Zoppe JO, Peresin MS, Habibi Y, Venditti RA, Rojas OJ (2009) Reinforcing poly ( $\varepsilon$ caprolactone) nanofibers with cellulose nanocrystals ACS applied materials \& interfaces 1:1996-2004 


\section{CHAPTER 6: NANOCELLULOSE-BASED DRUG DELIVERY SYSTEM - A REVIEW}

\subsection{Introduction}

Drug release refers to the process in which drug solutes migrate from the initial position in the polymeric system to the polymer's outer surface and then to the release medium. This process is affected by multiple complex factors including material matrix (composition, structure, swelling, degradation), release medium ( $\mathrm{pH}$, temperature, ionic strength, enzymes), and drug compounds (solubility, stability, charges, interaction with matrix) (Azhar et al. 2017).

Controlled release systems are being developed to deliver the needed amount of drug, to increase the effect of the drug in the body, to protect it from physiological degradation, to improve patient comfort and to be able to control the location where the drug is actually delivered. These systems are being used to achieve a spatial and time dependent control of delivery. Additionally, economic considerations related to the reduction in the frequency and the dose of the drug and the extension of the product life are the key factors behind research efforts in this field (Ulker and Erkey 2014).

Recently, the use of nanotechnology in drug delivery has attracted a lot of interest due to its potential for development of systems that are site specific and/or that offer time dependent controlled delivery (Ulker and Erkey 2014).Nanoparticles, nanocapsules or micelles as nanotechnology based drug delivery systems offer many advantages over the conventional delivery systems. Furthermore, the problems associated with the delivery of a free drug such as poor solubility, tissue damage on extravasation, rapid breakdown in vivo, unfavorable pharmacokinetics, poor bio-distribution, and lack of selectivity for target tissues can be overcome by designing drug delivery vehicles via nanotechnology (Ulker and Erkey 2014).

Choosing a suitable polymer that is biocompatible, able to encapsulate, control, and target the release of the drug and yet biodegradable is highly critical for the successful 
formulation of nanomedicine. The ability of nanomedicines to target specific sites depends upon the particle size, surface charge, surface modification, and hydrophobicity, which in turn determine their interaction with the cell membrane and their penetration across the physiological drug barriers (Kumari et al. 2010).

Nanoparticles have been used in drug deliverydue the fact that they exist in the same size domain as proteins. Their large surface areas can also allow for displaying a large number of surface functional groups such as ligands. Furthermore, they have a rapid absorption and release behavior provided by high abilities of their diffusion and volume change. In addition, the particle sizes and surface characteristics of nanoparticles can be tailored or controlled. In particular, biopolymers are suitable materials as nanoparticles for clinical application due to their versatile traits, including biocompatibility, biodegradability and low immunogenicity. Biopolymers are polymers that are produced from living organisms, which are classified in three groups: polysaccharides, proteins and nucleic acids. It is important to control particle size, charge, morphology of surface and release rate of loaded molecules to use biopolymer-based nanoparticles as drug/gene delivery carriers. To obtain a nano-carrier for therapeutic purposes, a variety of materials and preparation process has been attempted.

Cellulose derivatives such as ethylcellulose, methylcellulose, carboxymethyl cellulose, hydroxypropylmethyl cellulose and numerous other forms have been extensively used in pharmaceutical preparations (Jackson et al. 2011). One of the key purposes of using cellulose as excipients in drugs is to control the rate of drug release and achieve the right drug concentration. This natural polymer can also be cross-linked into hydrogels because of its affinity toward water. Additionally, cellulose and cellulose derivatives pass through the human body safely, and some of the derivatives can be broken down digestive enzymes into natural metabolites in the gastrointestinal tract (Ilevbare et al. 2013). Nanocellulose obtained from cellulose-the most abundant biopolymer on Earth-is an emerging renewable polymeric nanomaterial that holds promise in many different applications including food and pharmaceuticals (Sanga Pachuau 2015). 
Several drug-delivery systems based on nanocellulose materials for various pharmaceutical applications have been used in recent years. All the three different categories of nanocelluloses including bacterial cellulose (BC), cellulose nanocrystals (CNCs) (also called as cellulose nanowhiskers or nanocrystalline cellulose), and cellulose nanofibrils (NFCs) have been widely investigated in drug delivery systems. Also, BC can be potentially safe for use in intravenous applications since it can be purified using sodium hydroxide to the US Food and Drug Administration (FDA) acceptable range of endotoxin values for implants(<20 endotoxin units/device) (Abeer et al. 2014).

Pharmaceutical nanotechnology focuses on formulatingtherapeutically active agents in biocompatible nanoformssuch as nanoparticles, microparticles,tablets, aerogels, hydrogels,

and transdermal drug delivery systems. This These systems offer many advantagesin drug delivery, mainly focusing on improved safety andefficacy of the drugs, e.g. providing targeted delivery ofdrugs, improving bioavailability, extending drug effect intarget tissue, and improving the stability of therapeuticagents against chemical/enzymatic degradation (Azhar et al. 2017).

This chapter will discuss some of the current research activities in the application of BNC, CNC, and NFC in different for drug delivery system.

\subsection{Nanofibrillated cellulose in protein and gene therapy}

Nanofibrillar cellulose (NFC) has attracted a lot of attention in drug delivery recently as a natural, biodegradable, abundant polymer with excellent mechanical properties as well as low cost, biocompatibility, hydrophilicity, relative thermostabilization, high sorption capacity, and low cytotoxicity.Additionally, cellulose and cellulose derivatives pass through the human body safely, and some of the derivatives can be broken down digestive enzymes into natural metabolites in the gastrointestinal trac (Qiu and Hu 2013).

Nanofibers have a very large surface-area-to-volume ratio, as large as 1000 times that of a microfiber. This property has generated a significant amount of interest in the biomedical and pharmaceutical industries, particularly for drug delivery of poorly soluble drug substances (Sharma et al. 2015).Matrices based on NFC can be used for the release of 
bioactive agents, such as drug molecules in a controlled manner for predetermined and long periods of time.

One common method for NFC production is TEMPO oxidation of the amorphous cellulose surfaces which often peels off most of the cellulosic substrate by creating carboxyl group on surface which can be attached to gene and protein based drugs easily (Laukkanen et al. 2012).NFC is preferred to use rather than synthetic polymers since the synthetic polymers are not biologically compatible with body fluids and tissues occurring in human body (Jain 2008).The use of liposomes as therapeutic vectors for instance, was hampered by their toxicity and lack of knowledge about their biochemical behavior as it needs ligand or conjugated system to be directed for drug delivery (Jain 2008).

Nano fibrillated cellulose (NFC) can be used for providing a matrix for sustained delivery of bioactive agent(s), wherein matrix comprises nanofibrillated cellulose and at least one bioactive agent incorporated with NFC. The matrix may further comprise additives. Examples of additives are agents for controlling swelling, agents for controlling hydrophobicity, thickening agents, polymeric substances, pharmaceutically acceptable solvents, antioxidants, stabilizators and preservatives etc. In this context, nanofibrillated cellulose suitably acts as a carrier of said bioactive agent. The bioacative agent can be bound to or encapsulated in NFC matrix. The bioactive agents include, but are not limited to: hormones, steroids, contraceptive drugs, drugs for hormone replacement therapy, gene therapy substances, etc. (Laukkanen et al. 2012).

Due to the original structure of the native cellulose, NFC contains a high amount of hydroxyl groups at the fibers' surface, which are available for the formation of hydrogen bonds which makes it to be able to be functionalized and attached to different other genes and proteins.

Additionally, this group of nanoparticles have been long seen as a promising way to enhance the dissolution rates of poorly water-soluble drugs due to their larger surface area and increased saturation solubility (Zhang et al. 2013). 


\subsection{Nanocellulose in Nanoparticulate Drug Delivery}

Elongated nanoparticles have recently been shown to have distinct advantages over spherical ones in targeted drug delivery applications. In addition to their oblong geometry, their lack of cytotoxicity and numerous surface hydroxyl groups make cellulose nanocrystals (CNCs) promising drug delivery vectors (Dong et al. 2014).

Dong et al. reported the synthesis of folic acid-conjugated CNCs for the targeted delivery of chemotherapeutic agents to folate receptor-positive cancer cells. Folate receptormediated cellular binding/uptake of the conjugate was demonstrated on human (DBTRG05MG, H4) and rat (C6) brain tumor cells. Folate receptor expression of the cells was verified by immunofluorescence staining. Cellular binding/uptake of the conjugate by DBTRG-05MG, H4, and C6 cells was 1452, 975, and 46 times higher, respectively, than that of non-targeted CNCs (Dong et al. 2014).Their findings indicate that the covalent attachment of folic acid molecules to the surface of CNCs is an effective method for the targeting of CNCs to folate receptor-positive mammalian cells. Their lack of cytotoxicity coupled with the demonstrated specific, folate receptor-mediated cellular uptake makes folic acid-conjugated CNCs promising nanoparticles for the targeted delivery of chemotherapeutic agents to cancer cells.

One of the earliest reports on the application of acid-hydrolyzed CNCs was published in 2011 (Jackson et al. 2011). NCC crystallites, prepared by an acid hydrolysis method, were shown to have nanoscopic dimensions and exhibit a high degree of crystallinity. These crystallites bound significant quantities of the water soluble, ionizable drugs tetratcycline and doxorubicin, which were released rapidly over a 1-day period. When the CNCs were treated with cetyltrimethylammonium bromide (CTAB), a significant increase in zeta potential was observed, which bound significant quantities of hydrophobic anticancer drugs such as docetaxel, paclitaxel, and etoposide. These drugs were released in a controlled manner over a 2-day period. The NCC-CTAB complexes were found to bind to KU-7 bladder cancer cells was also observed.

A novel polyelectrolyte-macroion complex (PMC) between chitosan, a cationic polysaccharide, and cellulose nanocrystals (CNCs), anionic, cylindrical nanoparticles, for 
potential applications in drug delivery was also prepared (Wang and Roman 2011).The high molecular weight of commercial chitosan limits its oral bioavailability, reducing the potential for systemic cytotoxicity.The objectives of this study were to investigate the formation of PMCs from chitosan and CNCs at different CNC concentrations and mixing sequences and to determine the properties of the resulting PMC particles with regard to their potential application as a multiparticulate oral drug delivery system.

Another ionic nanocomplex prepared between cationic $\beta$-cyclodextrin $(\beta-C D)$ and the CNCs were also used to encapsulate curcumin for controlled drug delivery. The synthesis of curcumin-cyclodextrin/cellulose nanocrystals (CNCx) nano complexes was performed. $\mathrm{CNCx}$ were functionalized by ionic association with cationic b-cyclodextrin (CD) and $\mathrm{CD} / \mathrm{CNCx}$ complexes were used to encapsulate curcumin. Preliminary in vitro results showed that the resulting curcumin-CD/CNCx complexes exerted antiproliferative effect on colorectal and prostatic cancer cell lines, with IC50slower than that of curcumin alone (Ntoutoume et al. 2016).

In a study by Wang et al. a novel polyphosphoester-grafted CNC was developed by the "grafting onto" process through "click" reaction, which possessed a negatively charged surface suitable for binding doxorubicin as an anticancer drug and delivers it to the HeLa cells. The system showed a good biocompatibility to the studied cells, internalized through endocytosis, and exhibited an anticancer activity against HeLa cells where the drug released was caused by the disruption of the electrostatic interaction in the acidic environment inside the tumor cells (Wang et al. 2015b).The novel modified polyphosphoester-grafted $\mathrm{CNC}$ was found to show a good $\mathrm{pH}$ response, making it a promising delivery vehicle for anticancer drugs.

It was demonstrated that $\mathrm{CNC}$ was capable of binding significant quantities of ionizable water soluble antibiotics which were rapidly released completely within a day. When CNC was surface modified with cetyltrimethylammonium bromide (CTAB) which is a common cationic surfactant with a quaternary ammonium head and a C16 alkyl tail., significant quantities of non-ionized hydrophobic drugs were bounded and the drugs were released in a controlled manner over several days (Abitbol et al. 2014). 
Folic acid-conjugated CNC was synthesized by Dong et al. for active targeting of chemotherapeutic agents to folate receptor-positive cancer cells. The aminated CNCs were labeled with fluorescent FITC and then conjugated with folic acid ( Figure 6.1) (Dong et al. 2014).

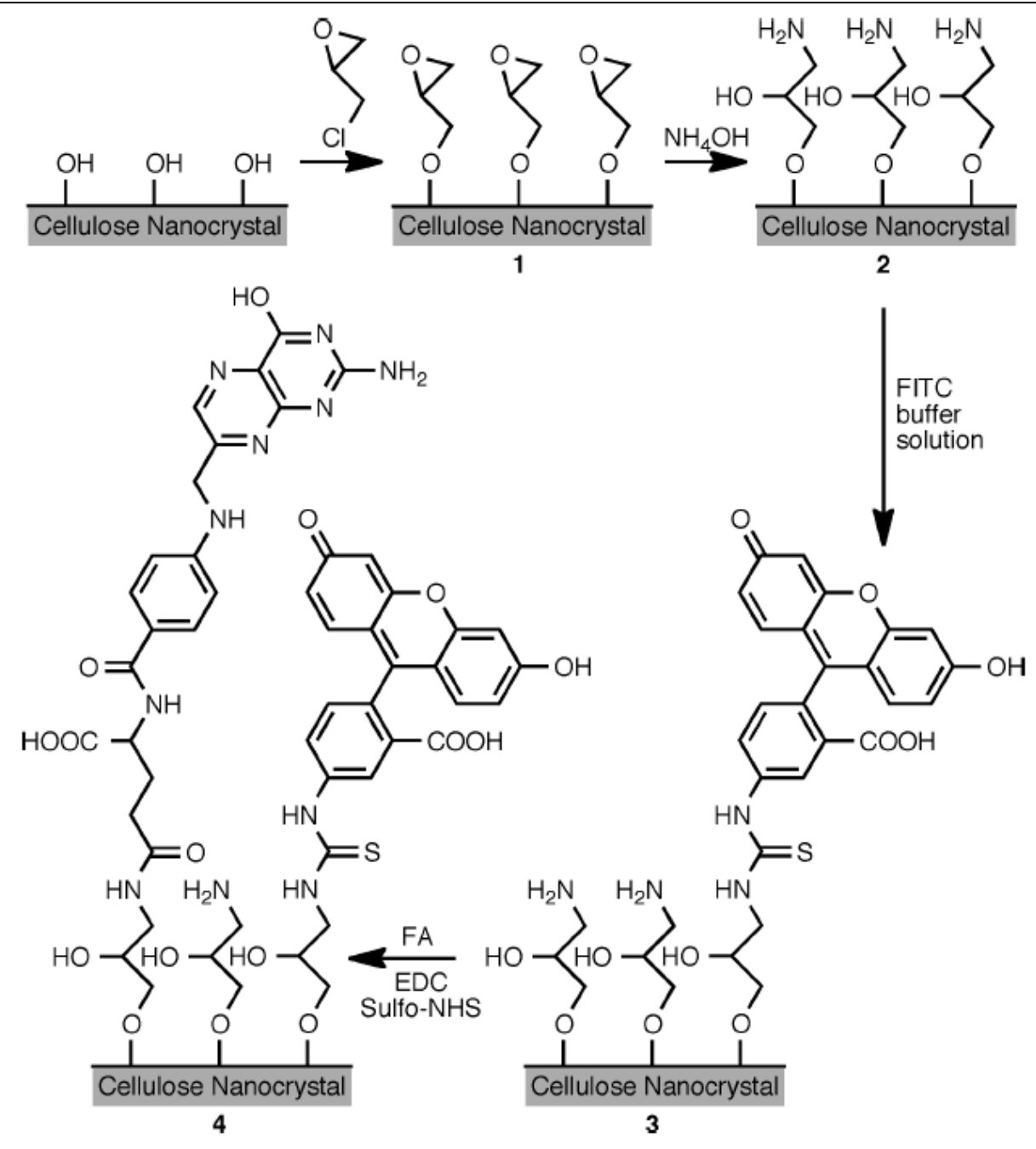

Figure 6.1 Synthesis of FITC-Labeled, Folic Acid-Conjugated CNCs (Dong et al. 2014).

Orelma et al. 2012 investigated the adsorption and chemical conjugation of avidin and its deglycosylated form, neutravidin, on films of regenerated and nanofibrillar cellulose.The installation of carboxyl groups on cellulose after modification with carboxymethylated 
cellulose (CMC) or TEMPO-oxidation significantly increased physisorption of avidins(Figure 6.2).The developed cellulose-avidin biointerfaces are able to scavenge biotinylated molecules from solution as demonstrated by successful surface complexation of biotinylated bovine serum albumin (Biotin-BSA) and antihuman immunoglobulin G (Biotin-anti-hIgG) (Orelma et al. 2012b).Avidin-biotin complexes have been widely employed in immunoassays, drug carriers and systems for linking antibodies, enzymes, and other molecules (Figure 6.3).

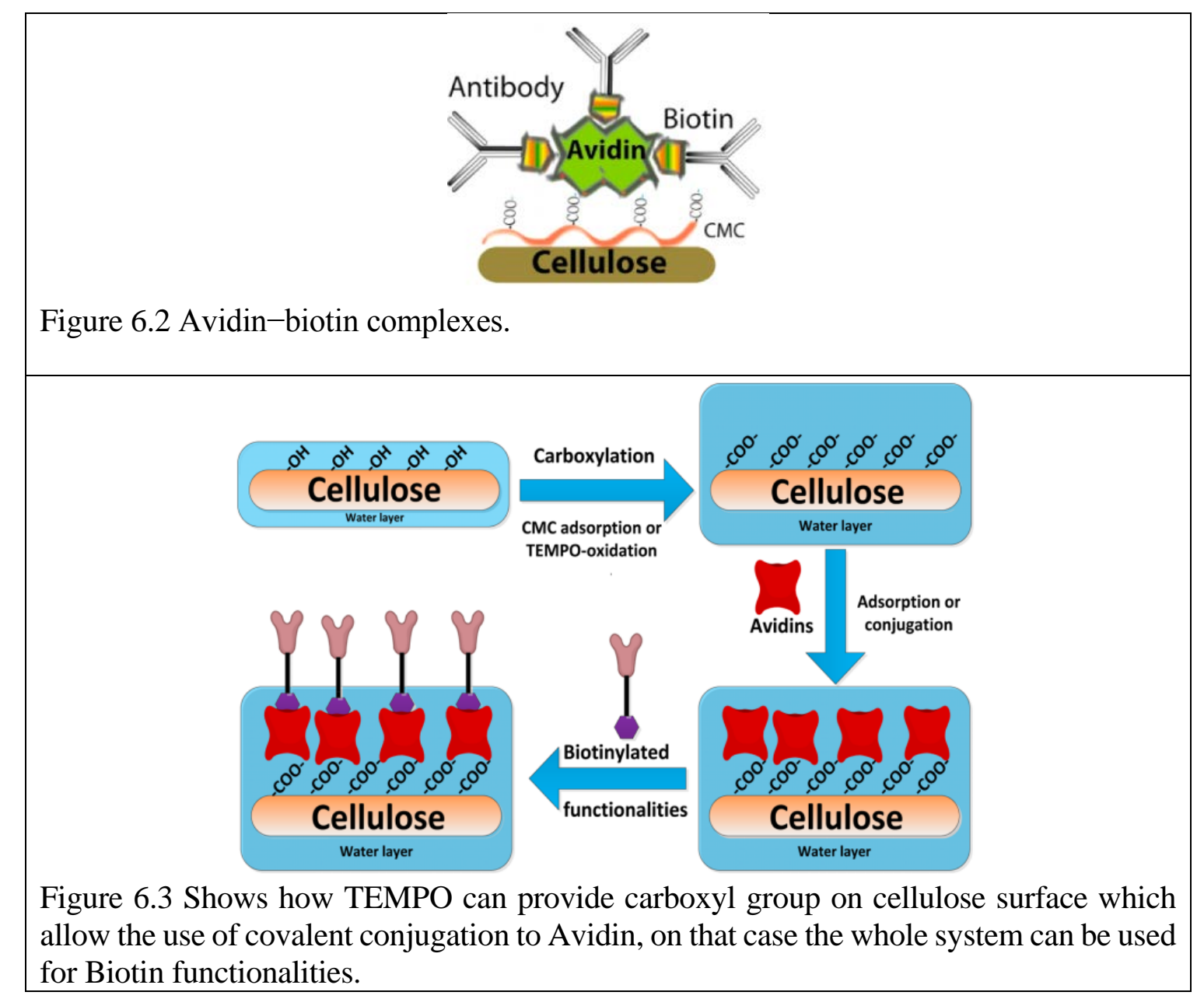

Kolakovic et al. also prepared films from NFCs for controlled drug delivery investigation (Kolakovic et al. 2012b) in which the NFC film was able to have more than $90 \%$ entrapment efficiency, with excellent mechanical properties. Dissolution studies showed a sustained the release of the drug for over three month's period with a very close to zero-order kinetics. 
These results indicate that NFC is a highly promising new material for sustained release drug delivery applications.

\subsection{Nanocellulose in gene therapy}

Gene therapy is a new kind of medicine, which uses genes as drugs in order to treat life threatening diseases.Gene delivery, the incorporation of foreign DNA particlesinto the host cells, is considered as one of the major objectivesin the development of nucleic acid therapeutics. Genetherapy is a suitable substitute for conventional proteintherapy because the problems such as bioavailability, systemictoxicity, manufacturing cost, and in vivo clearance rate canbe solved (Anirudhan and Rejeena 2014).Gene therapy shows much promise in tackling various geneticdiseases and cancers, viral infection, and cardiovasculardisorders (Xu et al. 2009).

Gene delivery can be mediated by viral and non-viral methods. In viral methods, viruses deliver genetic materials to the specific disease sites via their efficient inherent mechanism. Viral vectors can provide efficient transduction and high gene expression. However, the use of viral vectors is quite limited due to safety concerns such as production of immunogenic reactions or mutagenesis of transfected cells (Pal et al. 2009).

These have necessitated the design of non-viral vectors as potential alternative which produces less complexity.Nonviral vectors are attractive gene delivery systems for tumor gene therapy (Zhang et al. 2010). These have lowimmunogenicity, are easier to manufacture, have no signs of oncogenicity, and are relatively cheap compared to viral vectors (Eng and Elkordy 2012).

Natural polysaccharides are very suitable candidates for genedelivery, because they are renewable, nontoxic, biodegradable,and excellent biocompatible materials (Xu et al. 2009). Hydroxypropyl cellulose(HPC) is an example of a derivative of natural polysaccharide cellulose withboth water and organic solubility, where some of the hydroxylgroups of cellulose have been hydroxypropylated to formpropylene oxide groups. HPC materials have beenapproved by the United States Food and Drug Administration(FDA)and widely used in food and drug formulations. The reactivehydroxyl groups on HPC can be used as initiation 
sites forforming side chains to produce the comb-like copolymers vialiving radical polymerizations. The reactive hydroxyl groups on HPC can be used as initiation sites for forming side chains producing the comb-like Polycationic vectors. Polycations can spontaneously condense negatively charged DNA by electrostatic interaction into compact nanocomplexes and reduce the electrostatic repulsion between DNA and cell surfaces. Polycationic vectors can also protect plasmid DNA from enzymatic degradation by nucleases and facilitate cellular transfection.Comb-shaped cationic copolymers (HPDs) composed of long biocompatible hydroxypropyl cellulose (or HPC)backbones and short poly((2-dimethyl amino)ethyl methacrylate) (or P(DMAEMA)) side chains were prepared as gene vectors via atom transfer radical polymerization (ATRP) from the bromoisobutyryl-terminated HPCbiopolymers. The P(DMAEMA) side chains of HPDs can be further partially quaternized to produce the quaternary ammonium HPDs (QHPDs)( Figure 6.4).
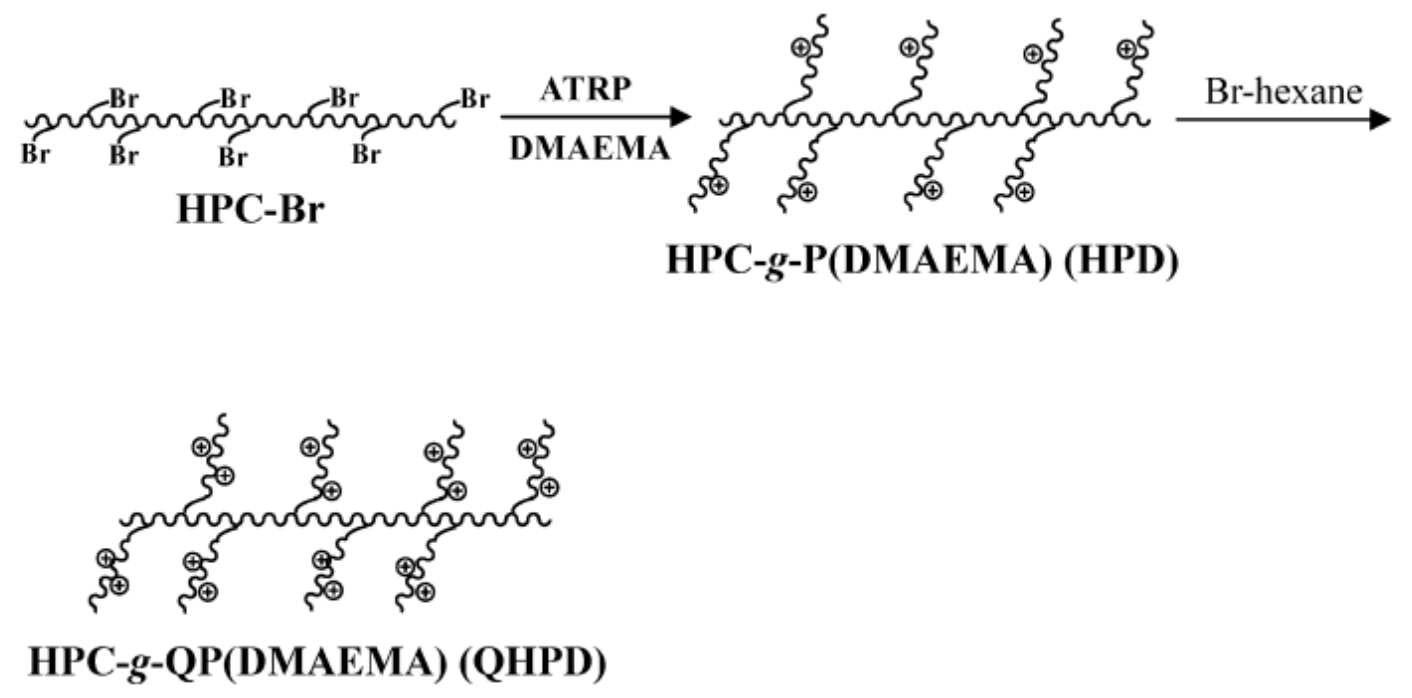

Figure 6.4 Schematic Diagram Ilustrating the preparation of cationic comb-shaped copolymers composed of HPC backbones. Comb-shaped poly-cationic vector can be considered as non-viral gene carriers (Xu et al. 2009).

Besides native cellulose, nanocellulose has retained the attention for this purpose because of renewability, low cost, and biodegradability; they present also the great advantage to be nontoxic. It has been recently shown that CNCs could target tumors via the EPR effect (Enhanced Permeability and Retention) and then deliver organic fluorescent compounds or 
drugs into cancer cells (Ntoutoume et al. 2017). As mentioned earlier, rod-like nanoparticles seem to be more rapidly internalized than spherical ones such as silica, iron or gold nanoparticules, and a recent work showed that rod-like nanoparticles have a significant impact on gene transfection (Lin et al. 2015).

Recently, Hu et al. reported the use of modified CNCs for pDNA delivery in vitro as well as in vivo. In their work,CNCs from natural cotton wool were functionalized with disulfide bond-linked poly(2-(dimethylamino)ethyl methacrylate) (PDMAEMA) brushes for effective biomedical applications.The CNC-SS-PDs exhibited good transfection efficiencies and low cytotoxicities. The needlelike shape of CNCs had an important effect on enhancing transfection efficiency. CNC-SS-PD also demonstrated good activity in suppressing the growth of cancer cells and tumors (Hu et al. 2015).

Ntoutoume et al. 2017 have prepared, for the first time, new cellulose-derived nanoparticles designed for intracellular siRNA delivery. This new nanovector consists of cellulose nanocrystals, coated with a covalently attached PEI cationic polymer shell. These particles could be easily loaded with siRNA via electrostatic interactions. This siRNA (siRNA killer) is able to silence the expression of cell cycle genes and to induce cell death by apoptosis. Therefore, this study suggests that these CNCs-PEI are promising non-viral nanovehicles for siRNA delivery and for efficient anti-tumor strategy.

A recently developed technique termed magnetofectionhas been shown to be a powerful technology in gene delivery. In this method, the complexes of nucleic acids and theirvectors are combined with magnetic nanoparticles, so theycan be drawn and concentrated to the target cells by applyinga magnetic field (Anirudhan and Rejeena 2014). In addition to facilitating targeted gene delivery, the rapid sedimentation of the geneparticle complex onto the target area significantly reduces both the time and dose of the vector to achieve efficient transfection. Magnetofection was found to be less cytotoxic than standard transfection. In this context, a novel nonviral vector as a gene carrier, aminated cyclodextrin-modified-carboxylated magnetic cobalt/nanocellulose composites (ACDC- 
Co/NCC) was developed for the efficient transfection of genes into tumor cells ( Figure 6.5).The cytotoxicity of ACDC-Co/NCC was observed to be minimal.
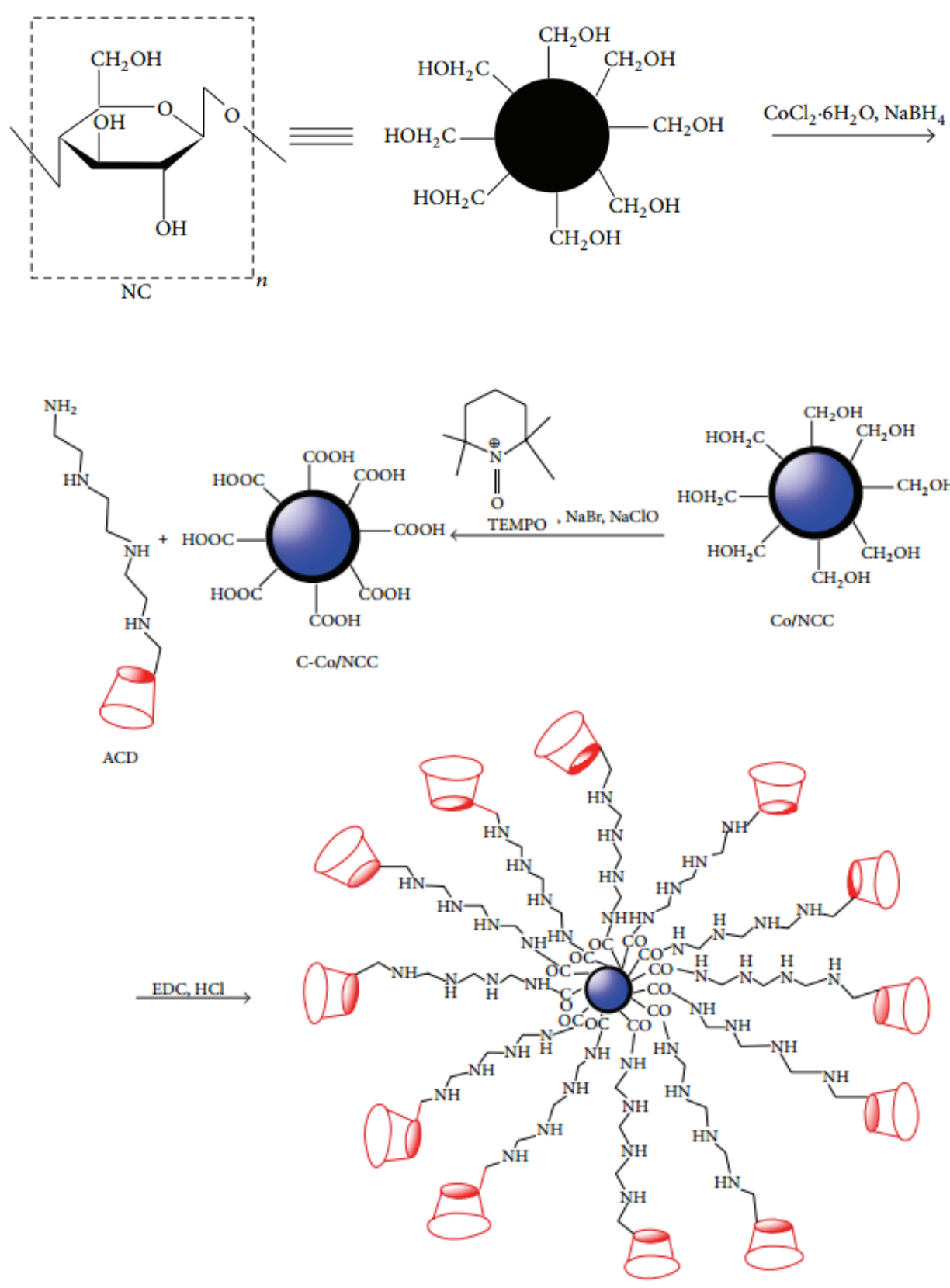

Figure 6.5 shows the developing of a non-viral gene vector, aminated $\beta$-cyclodextrin (ACD) modified carboxylated magnetic cobalt/nanocellulose composite (ACDC$\mathrm{Co} / \mathrm{NCC}$ ) for targeting the epithelial cells. 


\subsection{Nanocellulose in Microparticulate Drug Delivery}

Nanocellulose is an emerging natural polymer that has received considerable interest in recent years as the encapsulating polymer for drug delivery. It has also been widely investigated to enhance the mechanical properties and influence drug delivery behavior of microcapsules prepared with other natural polymers (Pachuau 2017).

A layer-by-layer (LbL) approach has been employed to create a multilayer thin films and microcapsules for controlled drug delivery. An electrostatic interaction between negatively charged CNCs and positively charged chitosan has been created. Both the water-soluble anticancer drug doxorubicin hydrochloride and lipophilic curcumin were successfully incorporated into the system for sustained drug delivery (Mohanta et al. 2014) (Figure 6.6).The amount of doxorubicin released at acidic $\mathrm{pH}$ ishigher, which iscommendatory for cancer therapy because of the lower extracellular $\mathrm{pH}$ of tumor cells. Though CNC is predominantly hydrophilic in nature, it consists of a surface showing higher hydrophobicity, and hence, it can interact with hydrophobic molecules. In this report, we have demonstrated that NCC can interact favorably with the water-insoluble drug e.g. curcumin, and the conjugates of curcumin with CNC form a stable dispersion in water

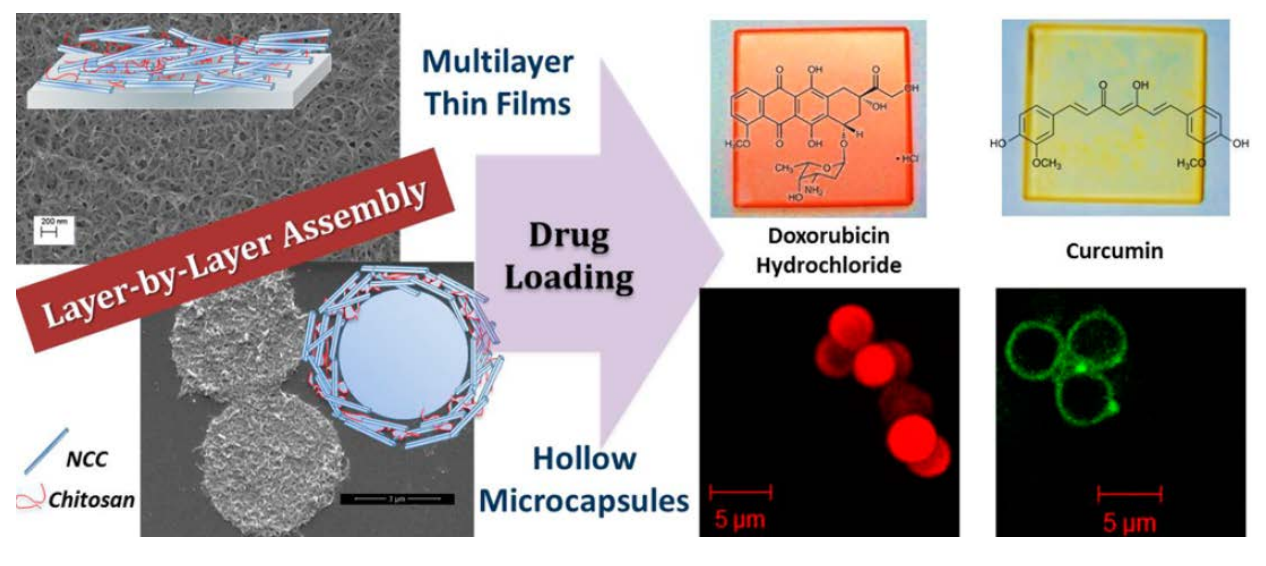

Figure 6.6 A layer-by-layer approach has for creating multilayer thin films and microcapsules for controlled drug delivery.

Cellulose nanofibers (NFCs) were isolated from poplar wood by using a mild chemical 
pretreatment combined with a high-intensity ultrasonication method. The nanocellulose and indomethacin structures were prepared for sustained release of the encapsulated drug (Gao et al. 2014) (

Figure 6.7). Indomethacin (IMC) is a nonsteroid anti-inflammatory drug that is practically insoluble in water. Given its low solubility, a high dose of IMC is usually administered to achieve a therapeutic effect, which leads to serious side effects. Thus, developing a vehicle for the controlled release of IMC is crucial to achieve sustained clinical medication (Gao et al. 2014). Herein, NFCs were used as templates for the nucleation and crystal growth of IMC to produce novel NFC/IMC composite structures. A high encapsulation efficiency of up to $97 \%$ of the water-insoluble drug indomethacin was achieved with drug release sustained over 30 days. Drug release was found to take place through diffusion, and fitting the drug release curves into various equations to determine the drug release mechanism showed that first-order model was the best fit. The organization of CNF/IMC composite fibers can be deduced as follows (Figure 6.8): First, the IMC molecules adhere to the CNF surfaces through bonds between hydroxyl and carboxyl groups. Subsequently, IMC nucleates and crystallizes on the surfaces of the CNFs. In the initial stage, the adhesion of IMC crystals on the surfaces prevents CNF self-aggregation. Next, the samples are treated by a homogenizer, and clustered CNF/IMC structures are obtained. The thin IMC coating on the surfaces of the CNFs is deemed as the nucleation site of theIMC crystals.During solvent evaporation, IMC starts to precipitateon the surfaces of the composite structures from the over-saturated solution, eventually forming a hierarchically orderedCNF/IMC structure. 


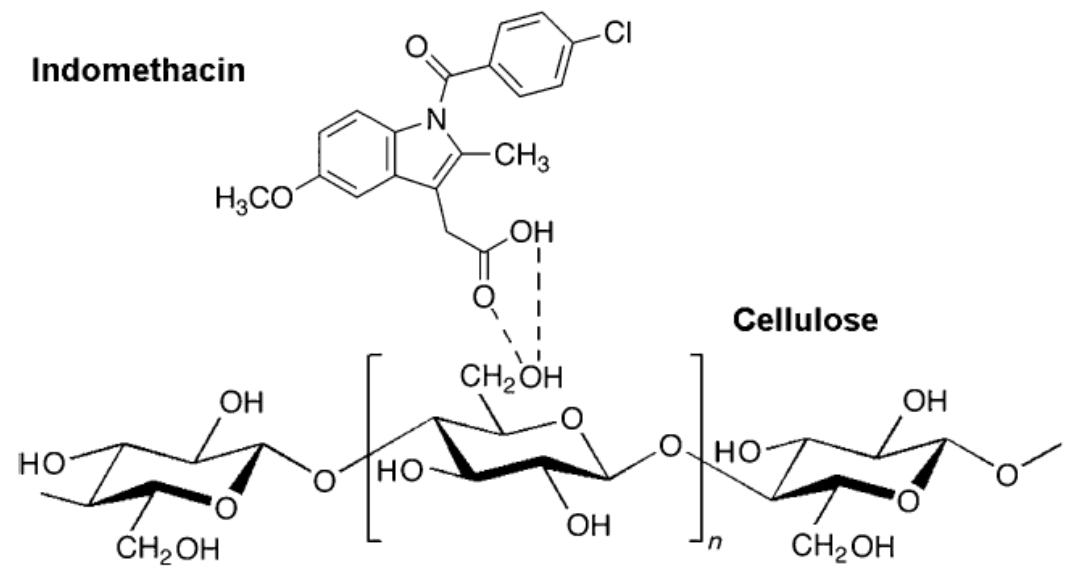

Figure 6.7 Chemical structures of IMC and cellulose (an essential componentof NFC), and the possible hydrogen-bonding mode.

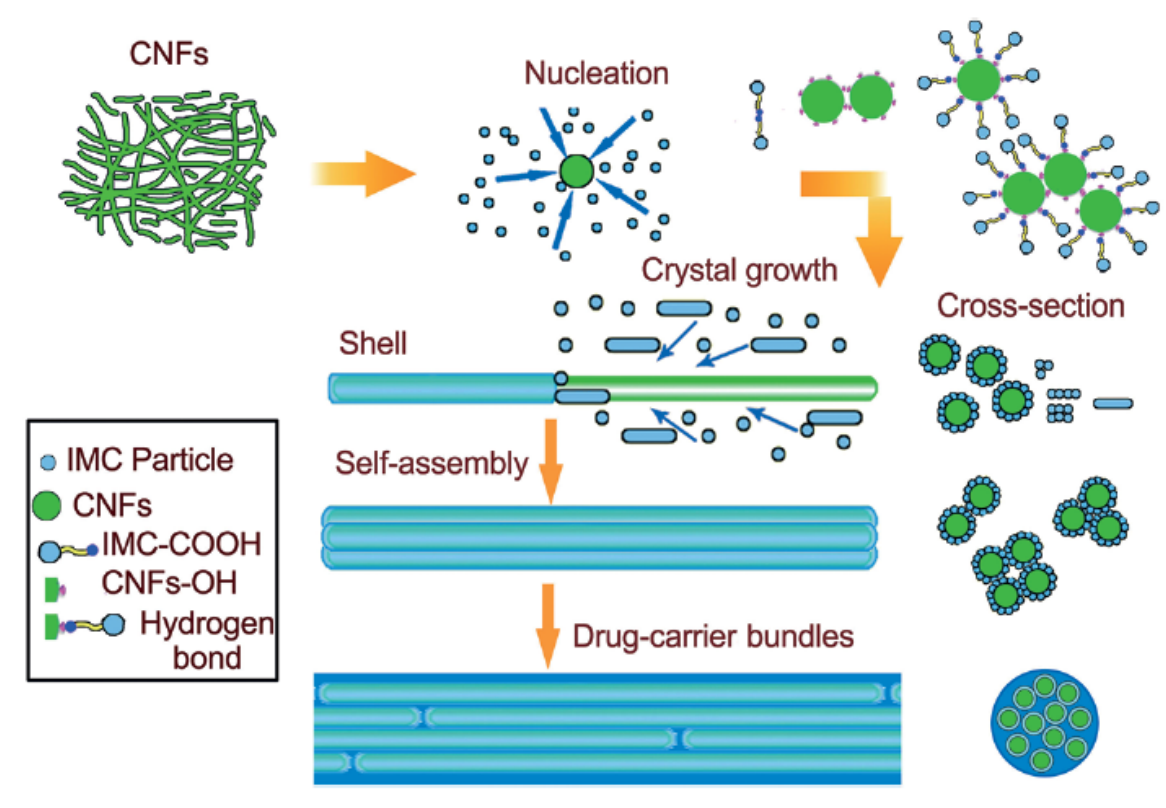

Figure 6.8 Schematic illustration of the formation of CNF/IMC composite fibers.

Matrix-type microparticles were prepared by spray drying of nanofibrillar cellulose for sustained delivery of different drugs such as indomethacin, nadolol, atenolol, metoprolol tartrate, verapamil hydrochloride, and ibuprofen (Kolakovic et al. 2012a). Spherical particles of diameters around $5 \mu \mathrm{m}$ were obtained by encapsulating the active drug mainly in the 
amorphous form. Final drug loading was quite low, up to $15.1 \%$ in indomethacin and $8.2 \%$ in verapamil hydrochloride. This low drug loading was attributed to the result of the low affinity between the drug and the cellulose fibers. When drug release from the microparticles was assessed by dissolution study, it was observed that after initial burstrelease, drug release was extremely slow taking over a 2-month period due to the tight binding between the drugs and the cellulose fibers. Drugswere released by diffusion through the matrix system, and when dissolutioncurves were fitted into different equations, different drugs were found to followdifferent release kinetics, mainly attributed to the inherent properties ofthe drugs. In another application in microencapsulation, nisin-loaded beads preparedwith alginateCNC were evaluated against its ability to inhibit growth of Listeria monocytogenes in ready to-eat (RTE) ham (Huq et al. 2014). Different concentrations of nisin (16, 31, and $63 \mathrm{lg} / \mathrm{ml}$ ) were microencapsulated into alginate-cellulose nanocrystal beads.Nisin-loaded beads were able to prevent the growth of the microorganism for at least 28 days.

Microparticles based on BC were also prepared by spray-drying method where the particles obtained were semispherical in shape. The resulting particles demonstrate good redispersibility, with better water retention capacity and higher thermal stability than microcrystalline cellulose $(M C C)$. The results also suggested that spray drying of acidtreated and mechanically processed BC powder improves its physical properties (Amin et al. 2014).

Beads from dissolved cellulose or cellulose beads (CBs)also showed to be a promising controlled delivery systemwhentailored to release the encapsulated drugs in a controlled manner (Yildir et al. 2013).

Weng et al. reported CMC microspheres with adjustable anticancer drug-release properties for potential arterial embolization applications. The as-prepared microspheres loaded with the anticancer drug doxorubicin showed a burst release profile in first $8 \mathrm{~h}$ followed by a plateau release over a 24-h period under physiological conditions. 
Although in vivo histological studies in the kidneys of rabbits after 6, 7, and 73 days of embolization revealed that the microspheres were biodegradable with only a mild tissue response (Weng et al. 2013)

Ernsting et al. developed docetaxel (DTX)-loaded CMC-based nanoparticles (NPs) for enhanced cytotoxicity against cancer cells. The carboxymethylcellulose polymer backbone has been used because of its biocompatibility and because it was reasonably stable with a proven safety profile, the CMC was acetylated to facilitate high hydrophobic drug coupling (37 wt \%) compared to other polysaccharide conjugates that have been reported these spherical NPs ( $120 \mathrm{~nm})$ released DTX at a slow and controlled rate of $3.8 \mathrm{w} / \mathrm{w} \%$ per day (100\% in 3 weeks). In vitro studies showed an enhanced cytotoxicity by $2-40$ fold compared to free DTX against cancer cells, presumably because of the slow release profile (Ernsting et al. 2011). Further in vivo antitumor evolution of these DTX releasing CMC NPs exhibited 90\% tumor growth inhibition compared with that of the native DTX (Roy et al. 2014).

In another study by Wen and Oh, dual-stimuli-responsive CMC-based nanogels were reported as potential intracellular anticancer drug-delivery carriers. In this study, oligo(ethylene oxide)-containing methacrylate (OEOMA) was polymerized in the presence of CMC and a disulfide-labeled dimethacrylate with free-radical crosslinking polymerization [FRCP; Figure 6.9].The in vitro intracellular release applicability of these nanocarriers containing the anticancer drug doxorubicin was confirmed with HeLa cancer cells (Wen and Oh 2014). These biopolymer-based nanogels (bionanogels) are a promising platform as polymer-based drug delivery systems encapsulting hydrophilic anticancer therapeutics 


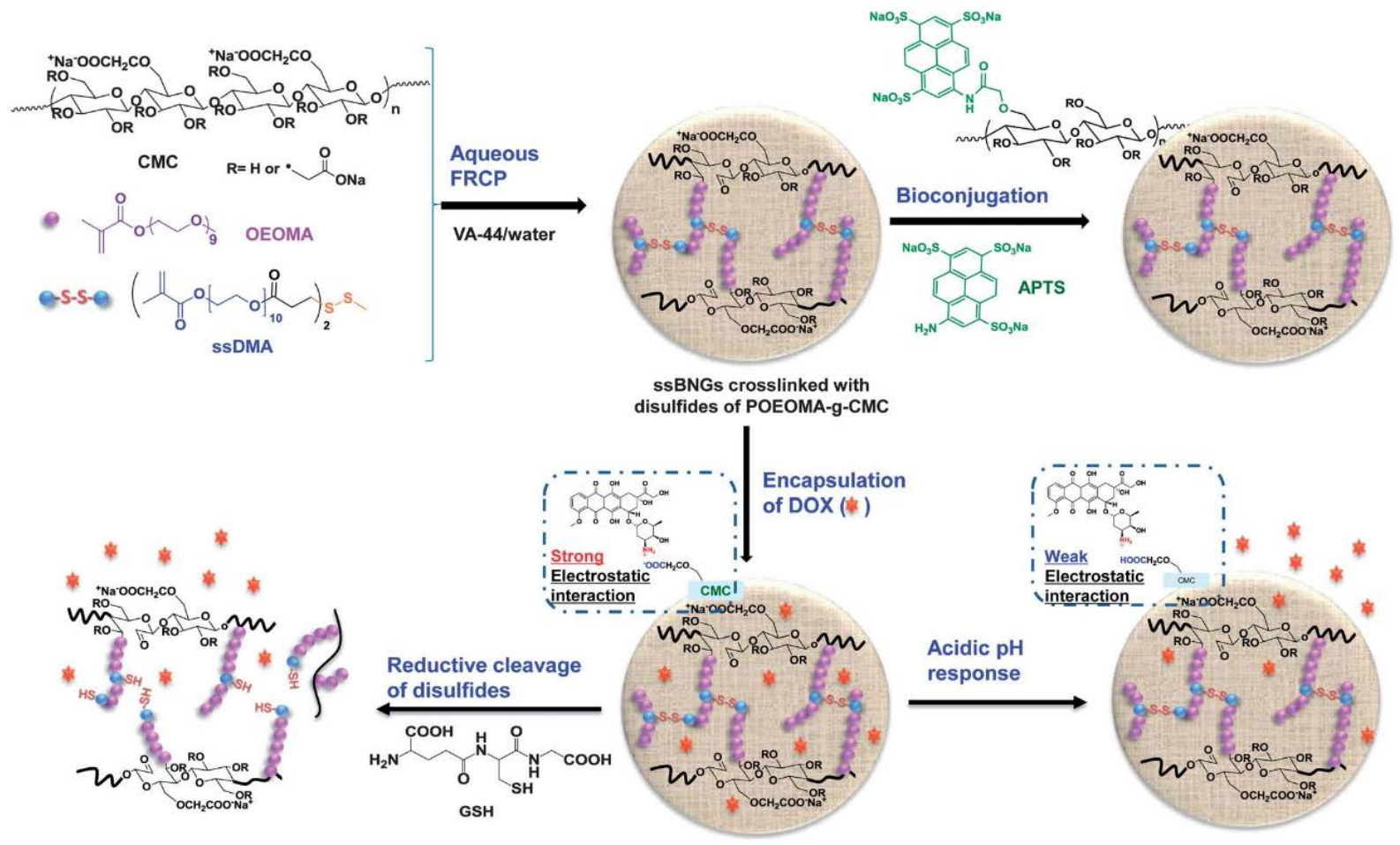

Figure 6.9 Synthesis by aqueous free radical crosslinking polymerization (FRCP), bioconjugation, and dual stimuli-response of biopolymer-based ssBNGs crosslinked with disulfide linkages of POEOMA-g-CMC (Wen and Oh 2014).

\subsection{Nanocellulose in Tablet Formulations}

With the development of new technology in pharmaceutical field, the emergence of novel pharmaceutical excipients has attracted extensive attention, among which efficient disintegrant has stood out. Solid dosage forms like tablets are most preferred and popular among the currently used drug delivery systems, because they have highly effective patient compliance, accurate dosing, good physical and chemical stability, and are convenient to produce, transport and market. These advantages result in low cost, high yield and being easy to achieve health standard (Wang et al. 2015a)

Cellulose derivatives such as MCC, hydroxypropyl methylcellulose, ethylcellulose, carboxymethylcellulose, and others are extensively used in conventional as well as controlled-release tablet formulations. With the practical edge provided by nanocellulose in numerous functional properties being realized and appreciated, a few investigations have 
explored its potential as functional excipients in tablet formulations. Disintegrants are added into tablet formulations to ensure that the tablet breaks up into fragments in the GI fluid tract to facilitate dissolution, which in turn results in enhanced bioavailability (Pachuau 2017). Nanocrystalline cellulose exhibited two potentially advantageous properties when used as disintegrant in calcium carbonate tablet preparation (Wang et al. 2015a). First is the reduced disintegration time and second is the increased hardness, which was observed with increase in the nanocrystalline cellulose concentration in the tablet formulation, confirming its potential disintegrant property.It is concluded that NCC exhibits novel fluidity and hydrophilicity that a potentially promising disintegrant possesses (Wang et al. 2015a).

If water is removed, hydrogen bonds are created between neighboring fibers, which create a tight fiber network. This process of inter-fiber binding is irreversible and is known in the literature as hornification. This property has been used to convert NFC aqueous suspension into a dry powder form by a spray drying method. Spray dried NFC particles can be used as an excipient for tablet production. Drug loaded spray dried microparticles have been recently produced for sustained drug release (Kolakovic 2013).

Spray-dried NFCs were also evaluated by Kolakovic et al. as novel tablet excipient and compared to two grades of commercial MCC Compared to commercial MCC, spray-dried NFC was found to possess good flow properties and better ability to pack with lower porosity. Dissolution study showed that formulations based on spray-dried NFCs releasedmaximum amount of its drug content in $10 \mathrm{~min}$. faster disintegration of the spraydried NFC tablets than Avicel PH 102 tablets has been recognized as plastic deformation during compaction was less pronounced with NFC powder resulting in the presence of fewer hydrogen bonds which caused faster tablet disintegration. Results from this study indicate the potential of NFCs to be used as an excipient for tablet production (Kolakovic et al. 2011).

Freeze-dried CNC prepared from water sugarcane bagasse was also shown to enhance the dissolution of diltiazem hydrochloride( DTZ) tablets prepared with the nanocellulose 
(Emara et al. 2014). Results showed that NCC might be a promising tablet excipient for enhancing drug dissolution of immediate release drug delivery systems. On the other hand, NCC could be used for controlling the release of freely water-soluble drugs (such as DTZ) by in-processdrug-loading within the NCC suspension.

\subsection{Nanocellulose in Transdermal Drug Delivery}

Transdermal drug delivery offers several advantages over traditional drug delivery systems such as oral delivery and injection, including elimination of hepatic first-pass metabolism, minimization of pain, and potential sustained release of drugs. However, because of the low permeability of the stratum corneum, different physical and chemical approaches have been developed to overcome the skin barrier and to have a better control of drug transport across the skin (Denet et al. 2003).Nanocellulose, especially bacterial cellulosic sources, has attracted a great deal of interest in the development of controlled transdermal drug delivery and wound healing preparations (Pushpamalar et al. 2016).

Huang et al. reported using bacterial cellulose membranes as vectors for the sustained release of berberine hydrochloride and berberine sulphate for its antibacterial and antiinflammatory properties. The drug carriers were also shown to have good sustained transdermal delivery (Huang et al. 2013b).

Trovatti et al. used BNC membranes as systems for topical release of lidocaine.In vitro drugrelease studies in a phosphate buffer solution $(\mathrm{pH} 7.4)$ at $32^{\circ} \mathrm{C}$ showed a burst release profile in which more than $90 \%$ of thetotal drug was released in the first 20 min (Trovatti et al. 2011).

Müller et al. investigated BNC as potential drug-delivery system for proteins with serum albumin. They found that the freeze-dried BNC samples showed a lower uptake of protein than the native BNC samples. Finally, Müller et al. showed that the biological stability of albumin was maintained during materials processing (Müller et al. 2013). 
The antiseptic octenidine was incorporated in bacterial nanocellulose with the intension to develop a ready-to-use system for wound treatment. The combination of both components leads to a wound dressing with advanced healing and superior material properties combined with an efficient infection control and minimized unwanted side effects. Octenidine loaded BNC demonstrated release profiles that were comparable to already marketed products. They were stable for up to 6 months storage without losing their favorable physicochemical and biological characteristics (Moritz et al. 2014).

BNC was also functionalized with the antiseptics povidone-iodine (PI) and polihexanide (PHMB) with the idea to supplementthe superior material properties of the nanostructuredbiopolymer as a wound dressing with antimicrobial activity.It could be demonstrated that theconcentration of loading solution and the BNC networkstructure are parameters that have a deep impact on theloading and release behavior, whereas alteration of thesurface structure due to cutting of BNC and the sampleshape could be neglected. Furthermore, the release mechanismwas neither affected by the type of antiseptic nor bythe sample dimensions (Wiegand et al. 2015).

A few research works on CNCs for transdermal delivery have also been dedicated in recent years. A transdermal delivery system for hydroquinone was developed to inhibit the production of melanin and prevent discoloration of the skin (Taheri and Mohammadi 2015). Hydroquinone-cellulose nanocrystal complex was prepared by incubating of hydroquinone solution in cellulose nanocrystals suspension.Sustained release of hydroquinone was achieved with $80 \%$ of the bound drug released in 4 h.It was demonstrated that CNC could bind in significant quantities to the hydrophilic drugs such as hydroquinone. The sustained release of hydroquinone from CNC-hydroquinone complex could demonstrate CNCs as suitable carriers for improving the efficacy and reducing the side-effects of drugs on the skin.

A novel drug delivery system based on two of the most abundant natural biopolymers was developed by modifying the surface of oxidized cellulose nanocrystal (CNC) with chitosan 
oligosaccharide (CSos).The in vitro drug release was studied at $\mathrm{pH} 8$ and the nanoparticles revealed a fast release of up to $1 \mathrm{~h}$, which can be used as biocompatible and biodegradable drug carriers for transdermal delivery applications (Akhlaghi et al. 2013).

Liu et al used regenerated cellulose micro-nanofiber matrices for transdermal drug release.The cellulose micro-/nano-fiber (CMF) matrices were prepared by electrospinning of pulp in an ionic liquid, 1-butyl-3-methylimidazolium chloride ([BMIM]Cl). A model drug, ibuprofen (IBU), was loaded on the CMF matrices by a simple immersing method.The spectra of FT-IR and TGA indicated that the drug was absorbed onto the CMF matrices effectively due to hydrogen bonding.From the in vitro release test, the IBU@CMF matrices showed bi-phasic release profiles and $80 \%$ of contained drug was released within $3 \mathrm{~h}$ (Liu et al. 2017).

\subsection{Hydrogels}

Hydrogels are three-dimensional (3D), cross-linked networks of water-soluble polymers which can be prepared by cross-linking of polymer chains through the interactions that may be of ionic, physical, or covalent, having the ability to absorb water (Azhar et al. 2017; Elisseeff 2008). In most cases, they are homogeneous materials, and their bulk properties are characterized and considered with regard to applications.

As drug delivery systems, increased use of hydrogel is based on its ability to origin a gel network in the swollen state which entraps the drug and acts as a barrier to itsrelease to the medium. In this way, the swelling ability of hydrogels is a fundamental property that should influence the drug release rates, by controlling both the diffusion rate of the penetrant into the matrix and the drug dissolution and diffusion throughout the gel layer of the swollen matrix (Azhar et al. 2017).

From a biomedical perspective, they show promise in a number of areas including devices, drug delivery and regenerative medicine (Elisseeff 2008).The design and preparation of hydrogels have attracted a great deal of interest in biomedical fields because of their tunable chemical and three-dimensional physical structure, good mechanical properties, high water 
content, and biocompatibility. These unique properties offer great potential for the utilization of hydrogels in tissue engineering, implants, drug delivery, and bionanotechnology (Azhar et al. 2017).

Hydrogels may display reversible sol-gel transitions, induced by changes in the environmental conditions such as temperature, $\mathrm{pH}$, ionic strength, phase separation, wave length of light, crystallinity, etc.Due to those abilities, smart polymeric hydrogels are extensively used in biomedical fields such as in the development of controlled-release drug delivery systems, tissue engineering, and regenerative medicine (Jagur-Grodzinski 2010).

The highly porous structure of hydrogels enables introduction of a relatively large load of drugs into them. The porosity of hydrogels can be tuned by controlling the density of crosslinks and their affinity toward the swelling environment. Their porosity also facilitates the release of drug at rates determined by their diffusion coefficient through the matrix of a hydrogel, which may be manipulated by changing the conditions of the environment (Jagur-Grodzinski 2010).

Nanocellulose derivatives have been used in thepreparation of biodegradable hydrogels both as mechanicalreinforcing and crosslinking agent. Among the various nanocellulose structures, cellulose nanofibers (CNF) are a promising choice for the preparation of hydrogels. Aside from its abundance and renewability, CNFdisplays high mechanical strength and stiffness, biocompatibility,a low percolation threshold and easily modification tointroduce functional groups or prepare cellulose derivatives (Azhar et al. 2017).

Hydrogels based on cellulose nanofibers and polyvinyl alcohol with different amounts of CNFs was synthesized as $\mathrm{pH}$ sensitive and controlled release drug delivery systems for cisplatin anticancer drug. cisplatin (cis diamminedichloroplatinum (II), CDDP) is one of the most widely used antineoplastic drugs for the treatment of various solid tumors including bladder, testicular, ovarian, head and neck, small cell and non-small cell lung cancers. Recently the application of biodegradable hydrogels for cisplatin delivery systems 
has attracted much attention. The porous hydrogel systems helped the absorption of water and making of a good carrier for controlled release of drugs (Azhar et al. 2017).

Injectable hydrogels are a subclass of hydrogels which can be prepared in situ by extruding hydrogel components from a syringe directly to the desired location in vivo. A new class of CNC-reinforced nanocomposites based on injectable polysaccharide hydrogels filled with CNCs that can act as simple fillers (unmodified CNCs) or as chemical cross-linkers (aldehyde-functionalized CNCs) has been prepared.No significant cytotoxicity to NIH 3T3 fibroblast cells was observed for the hydrogels or their individual components. These properties make CNC-reinforced injectable hydrogels of potential interest for variousbiomedical applications such as drug delivery vehicles or tissue engineering matrices (Yang et al. 2013).A biocompatible hydrogel with a double-membrane structure is developed from cationic cellulose nanocrystals (CNC) and anionic alginate. The architecture of the double-membrane hydrogel involves an external membrane composed of neat alginate, and an internal composite hydrogel consolidates by electrostatic interactions between cationic CNC and anionic alginate. Two drugs , Ceftazidime hydrate and human epidermal growth factor, were introduced into the different membranes of the hydrogel, which ensured the complexion drugs co-delivery and the varied drugs release behaviors from two membranes (rapid drug release of the outer hydrogel, and prolonged drug release of the inner hydrogel). (Lin et al. 2016). Controlled release lasting form more than 6 days was achieved for the incorporated drugs (Figure 6.10).

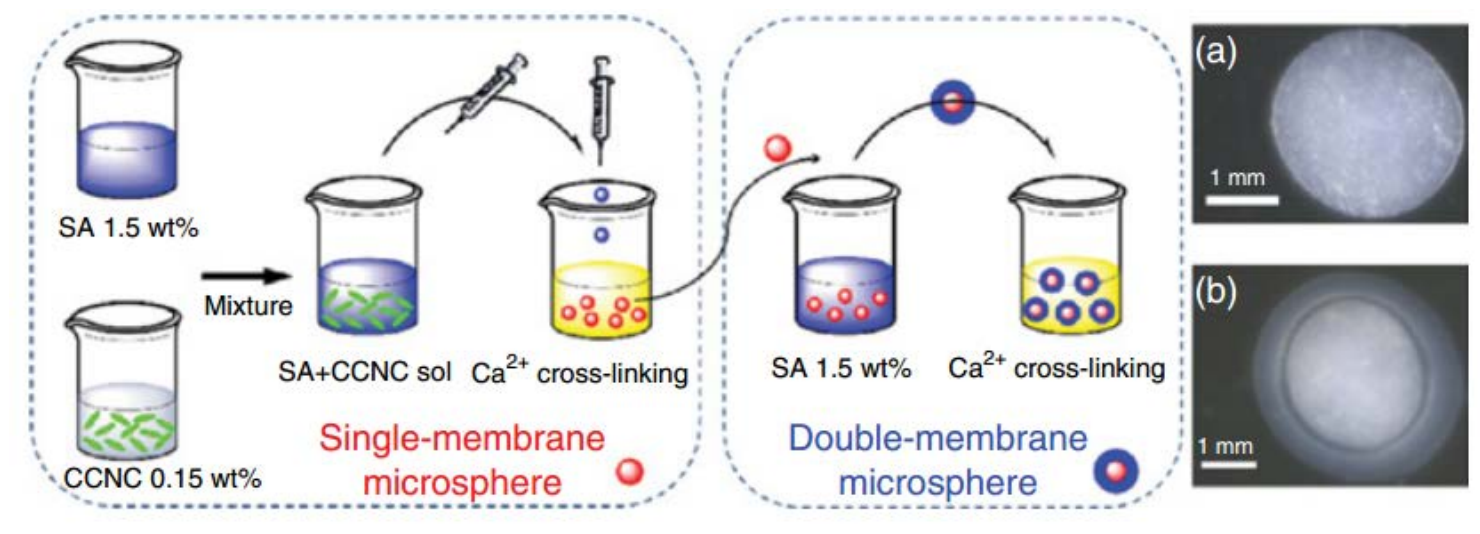

Figure 6.10 Preparation routine of single-membrane and double-membrane microsphere hydrogels; optical microscope images of (a) the Sodium alginate (SA)/CCNC single- 
membrane microsphere hydrogel and (b) the SA/CCNC-1h double-membrane microsphere hydrogel (Lin et al. 2016).

Cellulose fibers-gelatin structure for controlled release of nanocurcumin(nanoparticles of curcumin) to be used for wound dressing and antimicrobial applications was developed through a green process (Raghavendra et al. 2014).CNF-gelatin system impregnated with nanocurcumin (NCGCFs) was reported to be superior in its antimicrobial activity against $E$. coli and S. aureus when compared with the non-formulated curcumin-impregnated structure. The prepared NCGCFs NCGCFs have greatpotential for their utilization in wound/burn dressings as well as in the fabrication of antibacterial finishings and textiles.

TEMPO-oxidized bacterial cellulose (TOBC)-sodium alginate (SA) composites were prepared to improvethe properties of hydrogel for cell encapsulation. The fibrillated TOBCs mixed with SA were cross-linked in thepresence of $\mathrm{Ca}^{2+}$ solution to form hydrogel composites. The compression strength and chemical stability of the TOBC/SA composites were increased compared with the SA hydrogel, which indicated that TOBCperformed an important function in enhancing the structural, mechanical and chemical stability of thecomposites. Cells were successfully encapsulated in the TOBC/SA composites, and the viability of cellswas investigated. TOBC/SA composites can be a potential candidate for cell encapsulation engineering (Park et al. 2015).

Several other smart hydrogels such as shape-memory bacterial nanocellulose hydrogels , supramolecular hydrogels, temperature-sensitive hydrogels, and many others with potential for drug delivery have been developed, which were based on nanocellulose (Pachuau 2017).

Hydrogels with remarkable sensitivity toward changes in $\mathrm{pH}$ were prepared using gelatin reinforced with cellulose nanocrystals (CNCs). The increase in the overall crystallinity improved the storage modulus of the CNC-gelatin hydrogel from $122 \mathrm{~Pa}$ to $468 \mathrm{~Pa}$ by the addition of $25 \%$ CNC. The ability of the CNC-gelatin hydrogel to respond to different $\mathrm{pH}$ values along with its high dynamic mechanical stability suggested that CNC-gelatin ydrogels are promising candidates as drug carriers. results showed that 
the highest drug loading and drug release rate were achieved by a gelatin hydrogel reinforced with 5\% CNC. Gelatin hydrogels rein-forced with 15\% CNC, however, showed the best balance of drug loading and controlled drug release properties, which make it a good candidate for further study in a drug delivery system (Ooi et al. 2016)( Figure 6.11).

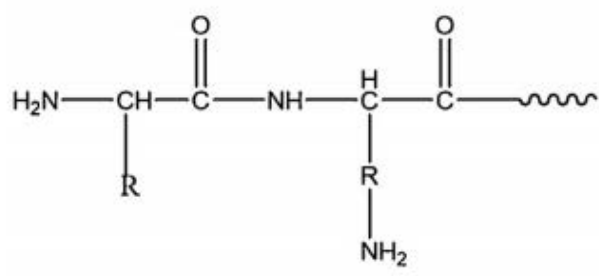

Gelatin

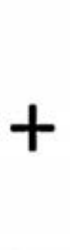

$\mid$

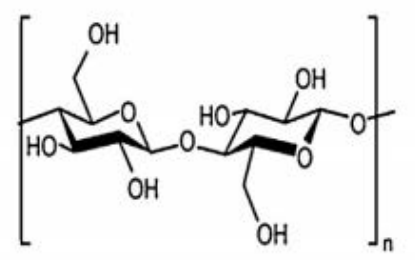

$\mathrm{CNC}$

$\mathrm{O}=\mathrm{CH}-\left(\mathrm{CH}_{2}\right)_{3}-\mathrm{CH}=\mathrm{O}$ (Glutaraldehyde)

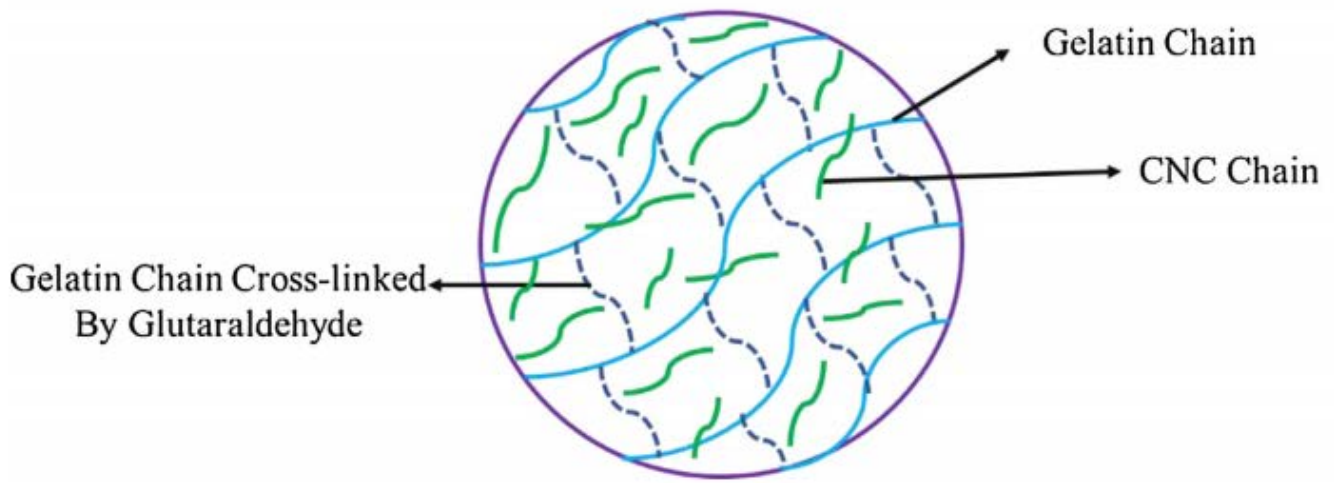

Figure 6.11 Proposed a mechanism for the formation of semi-interpenetrating polymer network (IPN) hydrogels.

A unique biodegradable, superporous, swellable and $\mathrm{pH}$ sensitive nanocellulose reinforced chitosan hydrogel with dynamic mechanical properties was prepared for oral administration of curcumin. Curcumin, a less water-soluble drug was used due to the fact that the fast swellable, superporous hydrogel could release a water-insoluble drug to a great extent. The maximum compression of pure chitosan hydrogel was $25.9 \mathrm{kPa}$ and it increased to $38.4 \mathrm{kPa}$ with the introduction of $0.5 \%$ cellulose nanocrystals.According to the results of this study, CNC reinforced chitosan hydrogel can be suggested to improve the bioavailability of 
curcumin for the absorption from stomach and upper intestinal tract (Udeni Gunathilake et al. 2017).

Dufresne's group reported one kind of supramolecular hydrogels based on cellulose nanocrystal by in situhost-guest inclusion. $\beta$-cyclodextrin was first grafted on cellulose nanocrystal, then the pluronic polymer and $\alpha$-cyclodextrin were attached by in situ inclusion, forming supramolecular hydrogels, as a drug carrier for doxorubicin.The doxorubicin can be released gradually in vitro, exhibiting the behavior of prolonged drug release (Xie and Li 2017).

Yang et al. presented a new class of CNC-reinforced nanocomposites based on injectable polysaccharide hydrogels filled with CNCs that could act as simple fillers (unmodified CNCs) or as chemical cross-linkers (aldehyde-functionalized CNCs). Based on their results, the product can be used in multiple biomedical applications, in particular, tissue engineering applications where longer term dimensional stability and enhanced mechanical strength are desirable (Yang et al. 2013)

\subsection{Aerogel Systems}

Over the past few decades, advances in "aerogel science" have provoked an increasing interest for these materials in pharmaceutical sciences for drug delivery applications. Because of their high surface areas, high porosities and open pore structures which can be tuned and controlled by manipulation of synthesis conditions, nanostructured aerogels represent a promising class of materials for delivery of various drugs as well as enzymes and proteins (Ulker and Erkey 2014).

Hydrogels may contain as much as $98-99 \%$ of water and only1-2\% of dry material. Nevertheless, they can maintain reasonablygood mechanical stability. Techniques have been developed toform aerogels from hydrogels by the removal of the water absorbedby them, without destroying their structure. Thus fabricated materialsfound applications in the food and pharmaceutical industries, whichmake good use of their enormous surface area (Jagur-Grodzinski 2010). 
Aerogels are highly porous materials that can have extremely low densities $(\sim 0.02 \mathrm{~g} / \mathrm{cm} 3)$ large open pores, and low thermal transport.They can be engineered for applications in catalysis, super adsorbents, elastic conductors, hydrogen and electrical energy storage, as well as desalination. To date, most aerogels are fabricated from silica, metal oxides, pyrolyzed organic polymers, and carbon-based materials. However, the traditional aerogels suffer from poor mechanical robustness, which has led to numerous attempts to overcome this problem (Chen et al. 2014).

Cellulose aerogels which are another type of organic aerogels are prepared either by freeze or supercritical drying of dissolved cellulose nanofibrils and nanowhiskers (Ulker and Erkey 2014).

Owing to web-like structure, high porosity, and high surface reactivity, the nanocellulose aerogels exhibited high mechanical flexibility and ductility, and excellent properties for water uptake, removal of dye pollutants, and the use as thermal insulation materials and drug delivery system. The aerogels also displayed sound-adsorption capability at high frequencies (Chen et al. 2014).As a result, different types of nanocelluloses, due to their excellent and suitable properties, have become the subject of keen interest in the preparation of aerogels for drug delivery.

A hydrophobic drug compound, itraconazole (ITR), was precipitated to form nanoparticles with either hydrophobins (HFBI or HFBII) or genetically modified fusion protein (HFBIDCBD). The nanofibrillar matrix provides protection for the nanoparticles during the formulation process and storage. It was demonstrated that by enclosing the functionalized protein coated itraconazole nanoparticles to the external nanofibrillar cellulose matrix notably increased their storage stability (Valo et al. 2011).

Highly porous nanocellulose aerogels prepared by freeze-drying from various nanofibrillar celluloses (NFC) hydrogels have been introduced as nanoparticle reservoirs for oral drug delivery systems. It has been shown that beclomethasone dipropionate (BDP) nanoparticles coated with amphiphilic hydrophobin proteins can be well integrated into the NFC aerogels. Freeze-drying method was applied to prepare highly porous aerogels from 
nanofibrillar cellulose obtained from four different sources and compared with MCC as nanoparticulate oral drug delivery systems (Valo et al. 2013). They observed that aerogels made from cellulose powder released the drug immediately; whereas the aerogels made from nanocelluloses (bacterial cellulose) showed sustained drug release. In another word, Release of the drug nanoparticle integrated into the aerogel system was found to be quick and immediate for red pepper-based aerogel and MCC, while BC,quince seed (QC), and TEMPO-oxidized birch cellulose-based (TC) aerogels show sustained drug release. A controlled release of the drug was achieved, which was modulated by the interactions between the drug nanoparticles and the cellulose matrix, making it a promising carrier for controlled drug delivery.

Dong et al. described three different systems such as hydrogels, aerogels, and films of NFCs which were functionalized with silver nanoparticles through the interaction of the negatively charged NFCs, obtained by TEMPO oxidation method and the positively charged silver, $\mathrm{Ag}^{+}$(Dong et al. 2013a). A stiff hydrogel was formed after the reaction, which was free-dried to obtain the aerogel with a potential for drug delivery applications.

In addition to plant cellulose, bacterial cellulose is another interesting source due to its high purity, molecular weight, fiber strength and degree of hydration and has already a 3D framework. Ultra-lightweight cellulose aerogels were prepared from purified bacterial cellulose as the starting material which was produced by the gram-negative bacterium Gluconacetobacter xylinum. The cultivated bacterial cellulose was briefly boiled, treated with $\mathrm{NaOH}$ and then washed with deionized water for neutralization. Resulting hydrogels were also subjected to solvent exchange step prior drying. The use of supercritical drying conserved the dimensional stability of the materials leading to very small amounts of shrinkage (Ulker and Erkey 2014). 


\subsection{References}

Abeer MM, Amin M, Iqbal MC, Martin C (2014) A review of bacterial cellulose-based drug delivery systems: their biochemistry, current approaches and future prospects Journal of Pharmacy and Pharmacology 66:1047-1061

Abitbol T, Marway H, Cranston ED (2014) Surface modification of cellulose nanocrystals with cetyltrimethylammonium bromide Nord Pulp Pap Res J 29:46-57

Akhlaghi SP, Berry RC, Tam KC (2013) Surface modification of cellulose nanocrystal with chitosan oligosaccharide for drug delivery applications Cellulose 20:1747-1764

Amin MCIM, Abadi AG, Katas H (2014) Purification, characterization and comparative studies of spray-dried bacterial cellulose microparticles Carbohydrate polymers 99:180-189

Azhar FF, Shahbazpour E, Olad A (2017) pH sensitive and controlled release system based on cellulose nanofibers-poly vinyl alcohol hydrogels for cisplatin delivery Fibers and Polymers 18:416-423

Chen W et al. (2014) Comparative study of aerogels obtained from differently prepared nanocellulose fibers ChemSusChem 7:154-161

Denet A-R, Ucakar B, Préat V (2003) Transdermal delivery of timolol and atenolol using electroporation and iontophoresis in combination: a mechanistic approach Pharmaceutical research 20:1946-1951

Dong H, Snyder JF, Tran DT, Leadore JL (2013) Hydrogel, aerogel and film of cellulose nanofibrils functionalized with silver nanoparticles Carbohydrate polymers 95:760767

Dong S, Cho HJ, Lee YW, Roman M (2014) Synthesis and cellular uptake of folic acidconjugated cellulose nanocrystals for cancer targeting Biomacromolecules 15:15601567

Elisseeff J (2008) Hydrogels: structure starts to gel Nature materials 7:271-273

Emara L, El-Ashmawy A, Taha N, El-Shaffei K, Mahdey S, El-Kholly H Freeze-dried nanocrystalline cellulose derived from water sugar-cane bagasse as a novel tablet excipient. In: The 41st Annual Meeting \& Exposition of the Controlled Release Society, Chicago, Illinois, USA, 2014. pp 13-16

Eng M, Elkordy A Gene delivery using non-viral vectors (cyclodextrins) with Pluronic-F127 and folic acid. In: Proceedings of the Second Electronic Conference on Pharmaceutical Sciences, Sciforum Electronic Conferences Series, 2012. pp 1-39

Ernsting MJ, Tang W-L, MacCallum N, Li S-D (2011) Synthetic modification of carboxymethylcellulose and use thereof to prepare a nanoparticle forming conjugate of docetaxel for enhanced cytotoxicity against cancer cells Bioconjugate chemistry 22:2474-2486 
Gao J, Li Q, Chen W, Liu Y, Yu H (2014) Self-Assembly of Nanocellulose and Indomethacin into Hierarchically Ordered Structures with High Encapsulation Efficiency for Sustained Release Applications ChemPlusChem 79:725-731

Hu H, Yuan W, Liu F-S, Cheng G, Xu F-J, Ma J (2015) Redox-responsive polycationfunctionalized cotton cellulose nanocrystals for effective cancer treatment ACS applied materials \& interfaces 7:8942-8951

Huang L, Chen X, Nguyen TX, Tang H, Zhang L, Yang G (2013) Nano-cellulose 3Dnetworks as controlled-release drug carriers Journal of Materials Chemistry B 1:2976-2984

Huq T, Riedl B, Bouchard J, Salmieri S, Lacroix M (2014) Microencapsulation of nisin in alginate-cellulose nanocrystal (CNC) microbeads for prolonged efficacy against Listeria monocytogenes Cellulose 21:4309-4321

Ilevbare GA, Liu H, Edgar KJ, Taylor LS (2013) Impact of polymers on crystal growth rate of structurally diverse compounds from aqueous solution Molecular pharmaceutics 10:2381-2393

Jackson JK, Letchford K, Wasserman BZ, Ye L, Hamad WY, Burt HM (2011) The use of nanocrystalline cellulose for the binding and controlled release of drugs International journal of nanomedicine 6:321

Jagur-Grodzinski J (2010) Polymeric gels and hydrogels for biomedical and pharmaceutical applications Polymers for Advanced Technologies 21:27-47

Jain KK (2008) Drug delivery systems, Humana Press (2008), pp. 1-50 vol 2. Springer,

Kolakovic R (2013) Nanofibrillar cellulose in drug delivery. Ph.D. Thesis. University of Helsinki, Helsinki, Finland

Kolakovic R, Laaksonen T, Peltonen L, Laukkanen A, Hirvonen J (2012a) Spray-dried nanofibrillar cellulose microparticles for sustained drug release International journal of pharmaceutics $430: 47-55$

Kolakovic R, Peltonen L, Laaksonen T, Putkisto K, Laukkanen A, Hirvonen J (2011) Spraydried cellulose nanofibers as novel tablet excipient Aaps Pharmscitech 12:1366-1373

Kolakovic R, Peltonen L, Laukkanen A, Hirvonen J, Laaksonen T (2012b) Nanofibrillar cellulose films for controlled drug delivery European Journal of Pharmaceutics and Biopharmaceutics 82:308-315

Kumari A, Yadav SK, Yadav SC (2010) Biodegradable polymeric nanoparticles based drug delivery systems Colloids and Surfaces B: Biointerfaces 75:1-18

Laukkanen A et al. (2012) Drug delivery system for sustained delivery of bioactive agents. Google Patents,

Lin N, Gèze A, Wouessidjewe D, Huang J, Dufresne A (2016) Biocompatible doublemembrane hydrogels from cationic cellulose nanocrystals and anionic alginate as complexing drugs codelivery ACS applied materials \& interfaces 8:6880-6889 
Lin X, Zhao N, Yan P, Hu H, Xu F-J (2015) The shape and size effects of polycation functionalized silica nanoparticles on gene transfection Acta biomaterialia 11:381392

Liu Y, Nguyen A, Allen A, Zoldan J, Huang Y, Chen JY (2017) Regenerated cellulose micro-nano fiber matrices for transdermal drug release Materials Science and Engineering: C 74:485-492

Mohanta V, Madras G, Patil S (2014) Layer-by-layer assembled thin films and microcapsules of nanocrystalline cellulose for hydrophobic drug delivery ACS applied materials \& interfaces 6:20093-20101

Moritz S et al. (2014) Active wound dressings based on bacterial nanocellulose as drug delivery system for octenidine International journal of pharmaceutics 471:45-55

Müller A, Ni Z, Hessler N, Wesarg F, Müller FA, Kralisch D, Fischer D (2013) The biopolymer bacterial nanocellulose as drug delivery system: investigation of drug loading and release using the model protein albumin Journal of pharmaceutical sciences 102:579-592

Ntoutoume GMN et al. (2016) Development of curcumin-cyclodextrin/cellulose nanocrystals complexes: new anticancer drug delivery systems Bioorganic \& medicinal chemistry letters 26:941-945

Ntoutoume GMN, Grassot V, Brégier F, Chabanais J, Petit J-M, Granet R, Sol V (2017) PEIcellulose nanocrystal hybrids as efficient siRNA delivery agents-Synthesis, physicochemical characterization and in vitro evaluation Carbohydrate polymers 164:258-267

Ooi SY, Ahmad I, Amin MCIM (2016) Cellulose nanocrystals extracted from rice husks as a reinforcing material in gelatin hydrogels for use in controlled drug delivery systems Industrial Crops and Products 93:227-234

Orelma H, Johansson L-s, Filpponen I, Rojas OJ, Laine J (2012) Generic method for attaching biomolecules via avidin-biotin complexes immobilized on films of regenerated and nanofibrillar cellulose Biomacromolecules 13:2802-2810

Pachuau L (2017) Application of Nanocellulose for Controlled Drug Delivery Nanocellulose and Nanohydrogel Matrices: Biotechnological and Biomedical Applications:1-19

Pal K, Banthia A, Majumdar D (2009) Polymeric hydrogels: characterization and biomedical applications Designed monomers and polymers 12:197-220

Park M, Lee D, Hyun J (2015) Nanocellulose-alginate hydrogel for cell encapsulation Carbohydrate polymers 116:223-228

Pushpamalar J, Veeramachineni AK, Owh C, Loh XJ (2016) Biodegradable polysaccharides for controlled drug delivery ChemPlusChem 81:504-514

Qiu X, Hu S (2013) “Smart” materials based on cellulose: a review of the preparations, properties, and applications Materials 6:738-781 
Raghavendra GM, Jayaramudu T, Varaprasad K, Ramesh S, Raju KM (2014) Microbial resistant nanocurcumin-gelatin-cellulose fibers for advanced medical applications Rsc Advances 4:3494-3501

Roy A, Murakami M, Ernsting MJ, Hoang B, Undzys E, Li S-D (2014) Carboxymethylcellulose-based and docetaxel-loaded nanoparticles circumvent Pglycoprotein-mediated multidrug resistance Molecular pharmaceutics 11:2592-2599

Sanga Pachuau L (2015) A mini review on plant-based nanocellulose: Production, sources, modifications and its potential in drug delivery applications Mini reviews in medicinal chemistry 15:543-552

Sharma J et al. (2015) Multifunctional nanofibers towards active biomedical therapeutics Polymers 7:186-219

Taheri A, Mohammadi M (2015) The use of cellulose nanocrystals for potential application in topical delivery of hydroquinone Chemical biology \& drug design 86:102-106

Trovatti E et al. (2011) Biocellulose membranes as supports for dermal release of lidocaine Biomacromolecules 12:4162-4168

Udeni Gunathilake TMS, Ching YC, Chuah CH (2017) Enhancement of curcumin bioavailability using nanocellulose reinforced chitosan hydrogel Polymers 9:64

Ulker Z, Erkey C (2014) An emerging platform for drug delivery: Aerogel based systems Journal of Controlled Release 177:51-63

Valo H et al. (2013) Drug release from nanoparticles embedded in four different nanofibrillar cellulose aerogels European Journal of Pharmaceutical Sciences 50:69-77

Valo $\mathrm{H}$ et al. (2011) Immobilization of protein-coated drug nanoparticles in nanofibrillar cellulose matrices—enhanced stability and release Journal of controlled release 156:390-397

Wang C, Huang H, Jia M, Jin S, Zhao W, Cha R (2015a) Formulation and evaluation of nanocrystalline cellulose as a potential disintegrant Carbohydrate polymers 130:275279

Wang H, He J, Zhang M, Tam KC, Ni P (2015b) A new pathway towards polymer modified cellulose nanocrystals via a "grafting onto" process for drug delivery Polymer Chemistry 6:4206-4209

Wang H, Roman M (2011) Formation and properties of chitosan- cellulose nanocrystal polyelectrolyte- macroion complexes for drug delivery applications Biomacromolecules 12:1585-1593

Wen Y, Oh JK (2014) Dual-stimuli reduction and acidic pH-responsive bionanogels: intracellular delivery nanocarriers with enhanced release RSC Advances 4:229-237

Weng L, Rostamzadeh P, Nooryshokry N, Le HC, Golzarian J (2013) In vitro and in vivo evaluation of biodegradable embolic microspheres with tunable anticancer drug release Acta biomaterialia 9:6823-6833 
Wiegand C et al. (2015) Antimicrobial functionalization of bacterial nanocellulose by loading with polihexanide and povidone-iodine Journal of Materials Science: Materials in Medicine 26:1

Xie J, Li J (2017) Smart drug delivery system based on nanocelluloses Journal of Bioresources and Bioproducts 2:1-3

$\mathrm{Xu} F$ et al. (2009) Comb-shaped copolymers composed of hydroxypropyl cellulose backbones and cationic poly ((2-dimethyl amino) ethyl methacrylate) side chains for gene delivery Bioconjugate chemistry 20:1449-1458

Yang X, Bakaic E, Hoare T, Cranston ED (2013) Injectable polysaccharide hydrogels reinforced with cellulose nanocrystals: morphology, rheology, degradation, and cytotoxicity Biomacromolecules 14:4447-4455

Yildir E et al. (2013) Tailored beads made of dissolved cellulose - Investigation of their drug release properties International journal of pharmaceutics 456:417-423

Zhang C, Gao S, Jiang W, Lin S, Du F, Li Z, Huang W (2010) Targeted minicircle DNA delivery using folate-poly (ethylene glycol)-polyethylenimine as non-viral carrier Biomaterials 31:6075-6086

Zhang Y, Nypelö T, Salas C, Arboleda J, Hoeger IC, Rojas OJ (2013) Cellulose nanofibrils Journal of Renewable Materials 1:195-211 


\section{CHAPTER 7: SAFETY ASPECTS ON THE UTILIZATION OF LIGNOCELLULOSIC BASED MATERIALS - A REVIEW}

\subsection{Introduction}

Lignocellulosic materials exhibit an interesting combination of mechanical properties and physical characteristics, which make them potentially useful for a wide range of applications. However, as the usage of these bio-based fibers increases, a greater understanding of human exposure addressing their potential health issues should be gained. This short report tried to underscore the current literature on the safety issues of nanocellulose and lignin- based biomaterials application. The nanocellulose materials showed lack of adverse health effects, whereas studies of the pulmoary and cytotoxicity have yielded discordant results. Studies the cytotoxic effects of lignin based biopolymers demonstrated the safety of lignin and lignocellulosic complexes regarding packaging materials and biomedical applications. Since there are not many works on this regard, additional studies are needed to assess and quantify the dose of the material's life-cycle, and to apply the realistic doses in contrast to overload situations in vivo target organs.

\subsection{Life-cycle of nanocellulose}

Several forms of nanocellulose, in their raw format, have been demonstrated to exhibit attractive property matrices and are potentially useful for the paper industry, as a reinforcing filler in polymer composites, basis for low-density foams, in packaging materials, additive in colloidal systems such as adhesives and paints, zerocalorie filler/thickener/stabilizer in a wide variety of food products, and in hygiene, cosmetic, and medical products (Endes et al. 2016).

There are five main points in the life-cycle of nanocellulose; i. isolation, ii. compounding, iii.productformation, vi. post-manufacturing processing and use, and v. Disposal (Figure 7.1). All stages of the life-cycle pose a potential human exposure scenario for which both the exposure level and the hazard associated, and thus the risk of CNCs to human health, are currently not fully understood (Camarero-Espinosa S. 2016). 


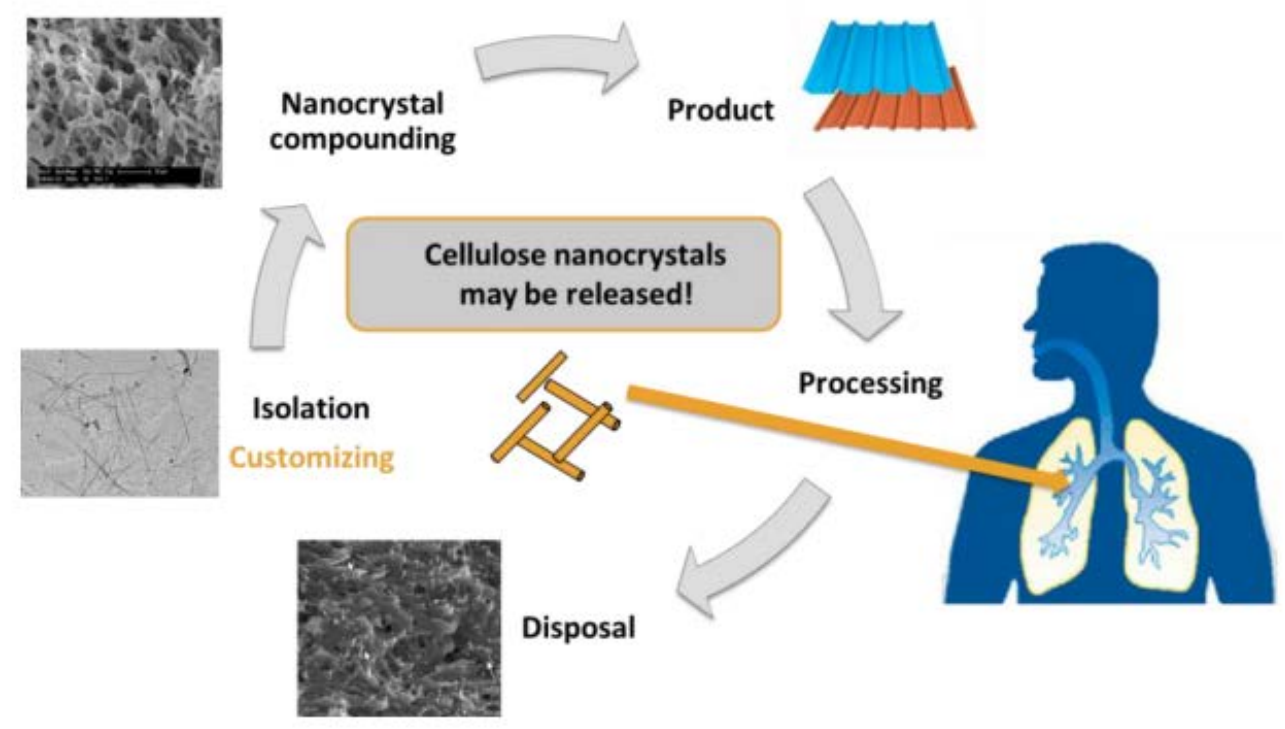

Figure 7.1 Schematic of the life-cycle of cellulose nanocrystals and products made from these nanoparticles (Camarero-Espinosa S. 2016).

A number of studies have recently been conducted to shed light on the aspect of potential biological impact of using nanocellulose. Some of the researches are discussed below:

\subsection{Biological impact of nanocellulose}

\subsubsection{Oral and dermal toxicity}

Current studies of the oral and dermal toxicity of CNCs have shown a lack of adverse health effects. The available studies, however, are still very limited in number (two oral toxicity studies and three dermal toxicity studies) and in the variety of tested CNC materials. Studies of pulmonary and cytotoxicity, on the other hand, have yielded discordant results (Moon et al. 2016; Roman M. 2015).

\subsubsection{Cytotoxicity effect of cellulose based nanoparticles}

One of the first important studies regarding the ecotoxicological impact of cellulose nanocrystals derived from 'kraft pulp' (CNC dimensions: $200 \times 10 \times 5 \mathrm{~nm}$ ) was published by Kovacs T. 2010, the authors summarized the outcome as "non-concerning". 
When investigated against nine different cell lines such as HBMEC, bEnd.3, RAW 264.7, MCF-10A, MDA-MB-231, MDA-MB-468, KB, PC-3, and C6 following the MTT and LDH assay methods, the filamentous CNCs showed no cytotoxic effects against any of these cell lines in the concentration range $\left(0-50 \mu \mathrm{g} \mathrm{ml}^{-1}\right)$ during the exposure time (48 h). Low nonspecific cellular uptake was observed, which indicates that CNCs are good candidates for nano drug delivery applications. (Dong et al. 2012).

When the biodegradability of CNC in aqueous environment was also studied as per the OECD standard and compared with other nanomaterials, CNCs and starch nanoparticles were found to biodegrade at similar levels but faster than their counterparts such as fullerenes and functionalized carbon nanotubes, which was attributed to their higher surface area (Kümmerer et al. 2011).

Further to this, several studies on cellulose-human interactions confirmed the limited toxic potential of nanocellulose in terms of cytotoxicity in various experimental systems.

A sophisticated triple-cell co-culture model of the human epithelial tissue barrier was used in a study that showed no significant cytotoxicity of two different CNC types isolated from cotton $(170 \pm 72 \times 19 \pm 7 \mathrm{~nm})$ and tunicates $(2.3 \pm 1.4 \mu \mathrm{m} \times 31 \pm 7 \mathrm{~nm})$ that were deposited onto the cells in realistic doses (Endes C. 2016).

However, there are also studies that have shown cytotoxic effects upon exposure to nanocellulose.

Mahmoud and co-workers investigated uptake and membrane integrity in human embryonic kidney cells (HEK 293) and Sf9 insect cells and found that exposure to $0.1 \mathrm{mg} /$ $\mathrm{mL}$ of negatively charged CNCs, led to membrane rupture under physiological $\mathrm{pH}$. Mahmoud and co-authors revealed that $(0.1 \mathrm{mg} / \mathrm{mL}$ CNCs elicit cytotoxicity in human embryonic kidney cells and ovary cells (Mahmoud et al. 2010), whereas Dong et al. declared that $0.01-0.05 \mathrm{mg} / \mathrm{mL}$ CNCs induce no measurable cytotoxicity in a wide range of barrier and immune cell types in vitro (Dong et al. 2012). 
Colic and co-authors showed that exposure to extremely high concentrations of long, entangled cellulose nanofibrils (NFC), lead to impaired metabolic activity and reduced cell proliferation (Čolić et al. 2015).

Recently it was reported that CNC may induce some slight dose-dependent cytotoxic and inflammatory effects on human lung cells, especially the risk ofinhalation exposure under high concentrations of released CNC powders (Clift MJ. 2011).

Regarding the toxicity of cellulose nanofibrils, Vartiainen J. 2011 assessed the immunotoxicity of MFC derived from ECF-bleached birch kraft pulp using cells from a mouse macrophage cell line and human peripheral blood-derived monocytes. The researchers found no evidence of inflammatory effects or cytotoxicity on either mouse or human cells (Vartiainen J. 2011).

Researchers in Finland showed low cytotoxicity and no DNA or chromosome damage from NFC, but pulmonary inflammation for mouse experiment possibly induced by the particulate/bacteria from NFC (Norppa H. 2012).

Pereira et al. evaluated the in vitro cytotoxicity and the effect on gene expression of NFC to fibroblasts cells. It was reported that low concentrations of NFC $(100 \mathrm{lg} / \mathrm{mL})$ have no obvious toxicity, whereas high concentrations of NFC (2000 and 5000lg/mL) will cause the sharp decrease of cell viability and affect the expression of stress- and apoptosisassociated molecular markers (Pereira M. 2013).

Alexandrescu et al. compared the cytotoxicity on fibroblast cells of pure NFC and surface modified-NFC with crosslinking agent polyethyleneimine (PEI) and surfactant cetyl trimethylammonium bromide (CTAB). In comparison with no acute toxic phenomena for pure NFC, both modified-NFC samples caused a significant reduction in cell viability and proliferation (Alexandrescu et al. 2013).

In another recent study, cationic modified-NFC (trimethylammonium-NFC) was reported to display a better cytocompatibility than unmodified and anionic modified-NFC (carboxymethylated-NFC)(Hua et al. 2014). 
Pitkanen et al. assessed the mutagenicity and cytotoxicity of both MFC made from birch pulp and NCC. Neither MFC nor NCC showed any evidence of mutagenicity or cytoxicity in these assays (Pitkänen et al. 2010).

In a rat acute oral toxicity test using NCC, NCC had no impact on survival or gross pathology. In a rabbit acute dermal irritation test, there were no signs of skin irritation following application of 0.5 grams NCC (O’Connor 2011).

The effect of nanocellulose-polymer polypyrrole (PPy) composite on cell viability was studied in vitro using fibroblast and monocyte cell lines, and in vivo in mice by intraperitoneal injection. The results indicated that the leachable of the composite did not induce any cytotoxic response in vitro or in vivo, but that the biocompatibility of the PPy composite depends largely on the rinsing and pre-treatment as well as the aging of the material.No cytotoxic effects were observed using 0.1-1wt\% NFC hydrogels in which nanofibrillar cellulose (NFC) isolated from bleached birch pulp (Stockmann P. 2014).

\subsubsection{Carcinogenicity and Inflammation study}

According to world health organization (2005), most cellulose fibers are not respirable and the hazard can be considered as low. Whereas, studies demonstrated that high dose of cellulose fibers when injected into the abdominal cavity of rats were tumorogenic (Votah J. 2009).

Inflammationwas reported based on dose of nanocellulose and its aggregation status: (Endes C. 2016)

Colic and co-workers showed an anti-inflammatory influence of cellulose nanofibril exposures on PBMCs (peripheral blood mononuclear cells) in vitro, which was only observed at considered high doses (0.25-1 mg/mL) (Čolić et al. 2015).

Catalan et al. 2015, exposed macrophage monocultures to cotton CNCs and found no detection of tumor necrosis factor(TNF) in comparison to microcrystalline cellulose; whereas, in an in vitro model of the human epithelial tissue barrier, it has been shown that in contrast to asbestos fibers, the exposure to CNCs does not induce a significant amount of tumor necrosis factor- $\alpha$ (TNF- $\alpha$ ) and interleukin-8 (IL-8) (Endes C. 2014). 
Despite the data discussed the above paragraph, there remains a prominent lack of coherent data to evaluate the potential of nanocellulose to pose a relevant hazard towards human health via an inflammatory immune response. Nevertheless, the existing studies point out that the physicochemical characteristics, especially the aggregation status of CNCs can have a detrimental impact towards elucidating a (pro-) inflammatory response. Moreover, overload exposures often mask the underlying specific mechanisms of toxicity and can only point in a general direction of a potential hazard (Endes C. 2016).

\subsubsection{Genotoxic influence of nanocellulose}

Only a few studies have so far investigated the genotoxicity of nanocellulose. Hannukainen et al. reported a potential genotoxic effect by the exposure of epithelial cells to NFC in 24h (Hannukainen et al. 2012).

Cotton and curaua nanofibers showed the ability to induce DNA strand breaks in human lymphocytes in concentrations of $0.1 \%$ of brown cotton and curaua fibers (de Lima et al. 2012). Kovacs et al. worked on primary rainbow trout hepatocytes and reported no changes in DNA quality after exposures to kraft pulp CNCs (Kovacs T. 2010).

Interestingly, a recent study reported the regulation of blood metabolic variables by the presence of TEMPO-oxidized cellulose nanofibers. The oral administration of TEMPOoxidized cellulose nanofibers to mice was proved to be effective for reducing the postprandial blood glucose, plasma insulin, glucose-dependent insulinotropic polypeptide, and triglyceride concentrations. It seems that TEMPO-oxidized cellulose nanofibers have both promising hemocompatibility and unique biological activities (Lin and Dufresne 2014).

A short overview on cytotoxicity, genotoxicity, and mutagenicity of cellulose based materials is presented in Table 7.1 
Table 7.1 An overview of the published studies focusing on the hazard assessment of nanocellulose(Camarero-Espinosa S. 2016).

\begin{tabular}{|c|c|c|}
\hline Nanocellulose Form Studied & Biological Model Used & Endpoint Assessed \\
\hline $\begin{array}{l}\text { Bacterial cellulose nanofibres } \\
\text { (BC-NF) }\end{array}$ & 3T3 fibroblasts, $\mathrm{CHO}$ cells & $\begin{array}{l}\text { mutagenicity, proliferation, } \\
\text { genotoxicity }\end{array}$ \\
\hline Bacterial cellulose nanofibres & HUVEC, C57/Bl6 mice & $\begin{array}{l}\text { viability, cytotoxicity, } \\
\text { apoptosis/necrosis, } \\
\text { cell cycle }\end{array}$ \\
\hline Cellulose nanocrystals (CNCs) & $\begin{array}{l}\text { Oncorhynchus mykiss hepatocytes, } \\
\text { Daphnia magna, Ceriodaphia dubia, } \\
\text { Pimephales promelas, Vibrio fischeri, } \\
\text { Pseudokirchneriella subcapitata, } \\
\text { Hydra attenuata, Danio rerio }\end{array}$ & $\begin{array}{l}\text { genotoxicity, reproduction, } \\
\text { survival, growth }\end{array}$ \\
\hline CNCs isolated from flay & HEK 293, Sf9 cells & uptake, cytotoxicity \\
\hline $\begin{array}{l}\mathrm{CNCs} \text { isolated from cotton } \\
\text { and tunicates }\end{array}$ & $\begin{array}{l}\text { 3D model of the pulmonary } \\
\text { epithelial airway barrier }\end{array}$ & $\begin{array}{l}\text { cytotoxicity, } \\
\text { (pro)inflammatory response }\end{array}$ \\
\hline $\begin{array}{l}\text { Cellulose nanofibers isolated } \\
\text { from caraua/cotton }\end{array}$ & $\begin{array}{l}\text { Allium cepa, primary lymphocytes, } \\
\text { 3T3 fibroblasts }\end{array}$ & Genotoxicity \\
\hline Plant derived CNCs & $\begin{array}{l}\text { HBMEC, bEnd.3, RAW 264.7, } \\
\text { MCF-10A, MDA-MB-231, } \\
\text { MDA-MB-468, KB, PC-3, C6 cells }\end{array}$ & uptake, cytotoxicity \\
\hline Nanofibrillated cellulose (NFC) & BEAS 2B cells & Genotoxicity \\
\hline $\begin{array}{l}\text { CNCs isolated from cotton, } \\
\text { flax, hemp }\end{array}$ & V79 fibroblast, Sf9 cells & Cytotoxicity \\
\hline $\begin{array}{l}\text { Cotton cellulose } \\
\text { nanofibres (CNF) }\end{array}$ & Bovine fibroblasts & $\begin{array}{l}\text { cytotoxicity, stress } \\
\text { response, apoptosis }\end{array}$ \\
\hline CNCs isolated from cotton & $\begin{array}{l}\text { BEAS 2B cells, monocyte-derived } \\
\text { macrophages }\end{array}$ & $\begin{array}{l}\text { cytotoxicity, genotoxicity, } \\
\text { inflammatory response }\end{array}$ \\
\hline CNCs isolated from MCC & NIH3T3 fibroblasts, HCT116 cells & cell viability \\
\hline CNFs isolated from cotton & Chlorella vulgaris & cell viability, growth \\
\hline
\end{tabular}

\subsection{Safety and regulatory issues of nanocellulose in food}

Wood pulp and powdered cellulose are generally recognized as being safe and can be used as a raw material for food contact materials or even as food additives.

However, in nanomaterials, the biological impacts on ecosystems and humans cannot be evaluated merely on their chemical characteristics. The size, shape, aggregation properties and different unknown factors might still affect the interactions of nanocellulose with cells and other living organisms (Serpa et al. 2016). 


\subsubsection{Oxidative stress potential}

Little is known about the radical forming potential of nanocellulose in cell-free and cellular environments, with studies mainly reporting insignificant impact on the oxidative stress status of the cells unless extremely high concentrations are applied ( like cotton NFCs on bovine fibroblasts), similar to the endpoints of cytotoxicity and inflammation.

In summary, it has been commonly reported that no significant oxidative stress is evident in vitro following nanocellulose exposure, i.e. using cotton CNCs $(0.14 \pm 0.04-1.57 \pm 0.03 \mu \mathrm{g} / \mathrm{cm} 2)$ in the human epithelial tissue barrier model, nanofibrillated celluloses $(9.5-950 \mu \mathrm{g} / \mathrm{cm} 2$ ) on bronchial cells, CNFs in high dose experiments with bovine fibroblasts and CNFs in lower doses to human fibroblasts (Endes C. 2016).

\subsubsection{Lignin-based materials}

Potential of the antimutagenic effect of lignin biopolymers have been demonstrated by several researchers:

Composites of lignin have been prepared and characterized as an alternative in food packaging system. In this regard, both lignosulfonate and sulfur-free lignin exhibited cytotoxic effect, but in a very high concentration. In another study, both unmodified and biologically modified lignin have been examined as preventive agents in cancer development and has been shown that these ligninexerted high DNA protection toward oxidative damage (Espinoza-Acosta et al. 2016).

Jankovic A. (2015) demonstrated that fabrication of linin into silver Hydroxyapatite (HA) can produce biofilms when subjected to different microorganisms exhibited low cytotoxicity toward human mesenchymal stem cells.

The incorporation of lignin to nanoscales materials have been investigated in different works which could enhance lignin antioxidant properties (Janković A. 2015).

In another study by Domenek et al ( 2013), the PLA Kraft lignin films were prepared and declared no toxic effect but increasing in antioxidant activity of the film by the generation of free phenolic monomers (Domenek et al. 2013). 
Lignin incorporated in Propylene film exhibited as a processing stabilizer and antioxidant. Additionally, nanoparticles containing lignin and chitosan were developed for cosmetic and biomedical purposes by Kim et al. 2013 which showed a high biocompatibility with human cells, and antimicrobial activity against E.coli and B.subtilis.

Fish gelatin lignin films have been prepared for packaging purposes for fish meat preservation in two different types of research, the incorporation of lignin improved the quality of the salmon and decreased lipid oxidation of the food (Espinoza-Acosta et al. 2016). Another research by Yearla and Padmassree (2016) revealed that analysis of the lignin incorporated into nanomaterials exhibited more antioxidant effect and UV protection compared to unmodified bulk lignin which can lead to promising application in pharmaceutical, food and cosmetic industries (Yearla and Padmasree 2016).

\subsection{Summary}

There is no evidence for serious influence or damage of nanocellulose on both cellular and genetic level as well as in vivo organ and animal experiments, However, the inhalation of plentiful nanocellulose (especially for CNC) may induce pulmonary inflammation due to the easy self-aggregation and non-degradation of nanocellulose in the body of animals.

Some of the observed cellular responses are the result of heavily overloaded systems and the outcomes, therefore, are deemed to be an effect of the dose and not the nanomaterials themselves.

Additionally, due to the nature of nanocellulose it is challenging to track it during uptake and fate due to a lack of analytical methods feasible to measure nanocellulose in biological systems.Therefore, the morphological impact or organ distribution after exposure is limited.

To summarize, the toxicity of cellulose nanomaterials will depend strongly on their physicochemical properties like surface chemistry, including particle charge, and degree of aggregation, which determines particle shape and dimensions. Therefore, these properties - which in turn depend strongly on the cellulose source, the preparation procedure, and post-processing or sample preparation methods, such as lyophilization, 
aerosolization, sonication, or sterilization-need to be carefully measured in the final samples. 


\subsection{References}

Alexandrescu L, Syverud K, Gatti A, Chinga-Carrasco G (2013) Cytotoxicity tests of cellulose nanofibril-based structures Cellulose 20:1765-1775

Camarero-Espinosa S (2016) Elucidating the potential biological impact of cellulose nanocrystals Fibers 4:21

Catalán J (2015) Genotoxic and immunotoxic effects of cellulose nanocrystals in vitro Environmental and molecular mutagenesis 56:171-182

Clift MJ (2011) Investigating the interaction of cellulose nanofibers derived from cotton with a sophisticated 3D human lung cell coculture Biomacromolecules 12:36663673

Čolić M, Mihajlović D, Mathew A, Naseri N, Kokol V (2015) Cytocompatibility and immunomodulatory properties of wood based nanofibrillated cellulose Cellulose 22:763-778

de Lima R (2012) Evaluation of the genotoxicity of cellulose nanofibers International Journal of Nanomedicine 7:3555

Domenek S, Louaifi A, Guinault A, Baumberger S (2013) Potential of lignins as antioxidant additive in active biodegradable packaging materials Journal of Polymers and the Environment 21:692-701

Dong S, Hirani AA, Colacino KR, Lee YW, Roman M (2012) Cytotoxicity and cellular uptake of cellulose nanocrystals Nano Life 2:1241006

Endes C (2016) A critical review of the current knowledge regarding the biological impact of nanocellulose Journal of Nanobiotechnology 14:78

Endes C (2014) An in vitro testing strategy towards mimicking the inhalation of high aspect ratio nanoparticles Particle and fibre toxicology 11:40

Espinoza-Acosta JL, Torres-Chávez PI, Ramírez-Wong B, López-Saiz CM, MontañoLeyva B (2016) Antioxidant, antimicrobial, and antimutagenic properties of technical lignins and their applications BioResources 11:5452-5481

Hannukainen K-S, Suhonen S, Savolainen K, Norppa H (2012) Genotoxicity of nanofibrillated cellulose in vitro as measured by enzyme comet assay Toxicology Letters 211:S71

Hua K, Carlsson DO, Ålander E, Lindström T, Strømme M, Mihranyan A, Ferraz N (2014) Translational study between structure and biological response of nanocellulose from wood and green algae RSC Advances 4:2892-2903

Janković A (2015) Structural and biological evaluation of lignin addition to simple and silver-doped hydroxyapatite thin films synthesized by matrix-assisted pulsed laser evaporation Journal of Materials Science: Materials in Medicine 26:1-14 
Kim S, Fernandes MM, Matamá T, Loureiro A, Gomes AC, Cavaco-Paulo A (2013) Chitosan-lignosulfonates sono-chemically prepared nanoparticles: characterisation and potential applications Colloids and Surfaces B: Biointerfaces 103:1-8

Kovacs T (2010) An ecotoxicological characterization of nanocrystalline cellulose (NCC) Nanotoxicology 4:255-270

Kümmerer K, Menz J, Schubert T, Thielemans W (2011) Biodegradability of organic nanoparticles in the aqueous environment Chemosphere 82:1387-1392

Lin N, Dufresne A (2014) Nanocellulose in biomedicine: Current status and future prospect European Polymer Journal 59:302-325

Mahmoud KA, Mena JA, Male KB, Hrapovic S, Kamen A, Luong JH (2010) Effect of surface charge on the cellular uptake and cytotoxicity of fluorescent labeled cellulose nanocrystals ACS applied materials \& interfaces 2:2924-2932

Moon RJ, Schueneman GT, Simonsen J (2016) Overview of Cellulose Nanomaterials, Their Capabilities and Applications JOM 68:2383-2394

Norppa H Nanofibrillated cellulose: results of in vitro and in vivo toxicological assays. In: Presentation on Sunpap conference, 2012.

O'Connor B Ensuring the safety of manufactured nanocrystalline cellulose: a risk assessment under Canada's new substances notification regulations. In: 2011 TAPPI international conference on nanotechnology for renewable materials, 2011.

Pereira M (2013) Cytotoxicity and expression of genes involved in the cellular stress response and apoptosis in mammalian fibroblast exposed to cotton cellulose nanofibers Nanotechnology 24:075103

Pitkänen M et al. (2010) Nanofibrillar cellulose-Assessment of cytotoxic and genotoxic properties in vitro. In: 2010 Tappi International conference on nanotechnology for the forest products industry, 2010.

Roman M (2015) Toxicity of cellulose nanocrystals: a review Industrial Biotechnology $11: 25-33$

Serpa A, Velásquez-Cock J, Gañán P, Castro C, Vélez L, Zuluaga R (2016) Vegetable nanocellulose in food science: a review Food Hydrocolloids 57:178-186

Stockmann P. (2014) Review on the toxicity of manufactured nanomaterials applied in the construction sector. Scaffold Public Documents Finnish Institute of Occupational Health (FIOH)

Vartiainen J. (2011) Health and environmental safety aspects of friction grinding and spray drying of microfibrillated cellulose Cellulose 18:775-786

Votah J (2009) A legal perspective on the environmental, health and safety aspects of nanomaterials. Presentation at TAPPI International Conference on Nanotechnology for the Forest Products Industry

Yearla SR, Padmasree K (2016) Preparation and characterisation of lignin nanoparticles: evaluation of their potential as antioxidants and UV protectants Journal of Experimental Nanoscience 11:289-302 